ERNEST DRLANDD LAWRENCE

BERKELEY NATIONAL LABDRATIRY

\title{
Site Environmental Report for 2007 Volume II
}

Environment, Health, and Safety Division

September 2008

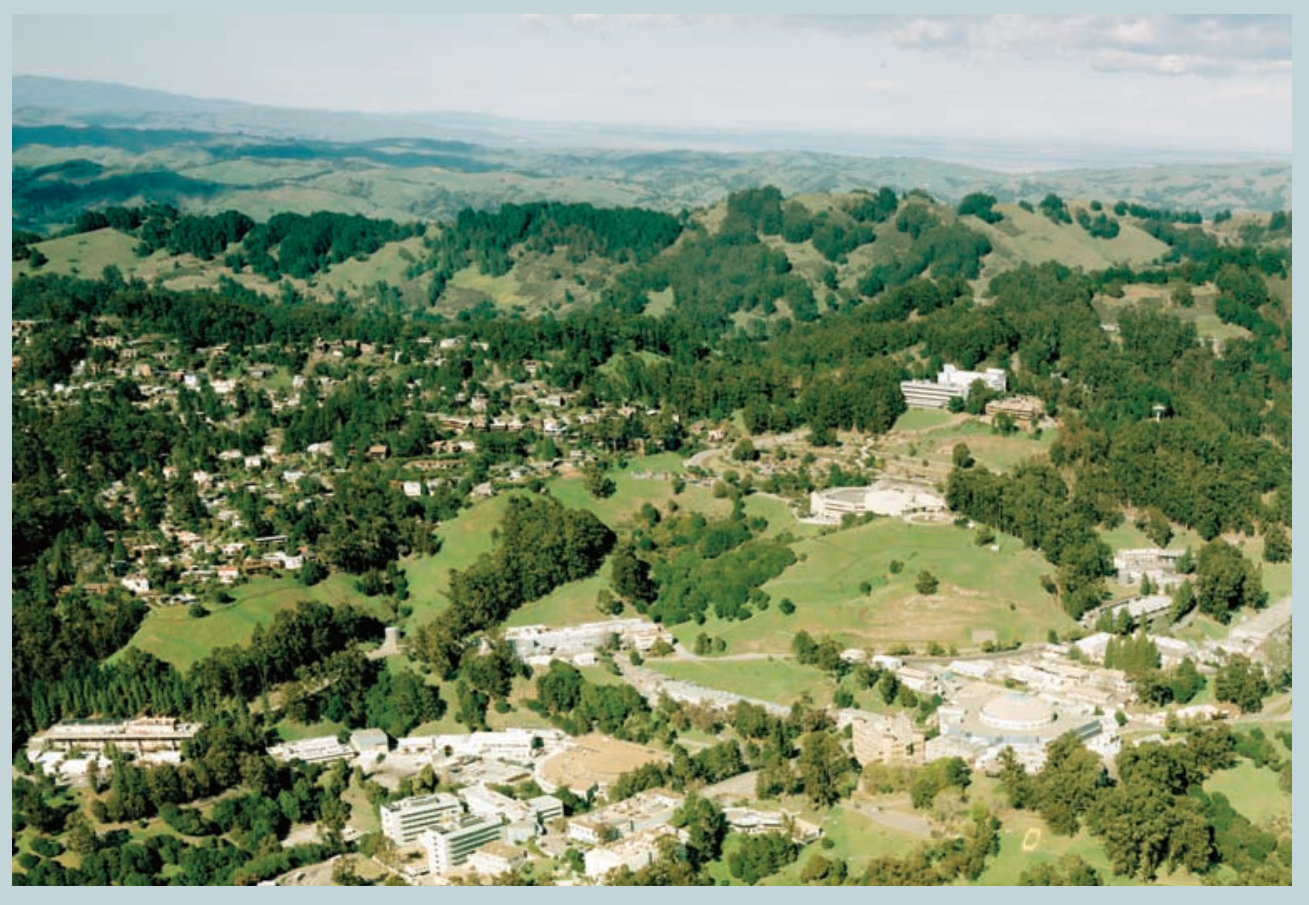




\section{DISCLAIMER}

This document was prepared as an account of work sponsored by the United States Government. While this document is believed to contain correct information, neither the United States Government nor any agency thereof, nor The Regents of the University of California, nor any of their employees, makes any warranty, express or implied, or assumes any legal responsibility for the accuracy, completeness, or usefulness of any information, apparatus, product, or process disclosed, or represents that its use would not infringe privately owned rights. Reference herein to any specific commercial product, process, or service by its trade name, trademark, manufacturer, or otherwise, does not necessarily constitute or imply its endorsement, recommendation, or favoring by the United States Government or any agency thereof, or The Regents of the University of California. The views and opinions of authors expressed herein do not necessarily state or reflect those of the United States Government or any agency thereof or The Regents of the University of California.

Ernest Orlando Lawrence Berkeley National Laboratory

is an equal opportunity employer. 

This work was supported by the Director, Office of Science, U.S. Department of Energy under Contract Number DE-AC02-05CH11231 


\title{
Site Environmental Report for 2007
}

\author{
Volume II
}

September 2008

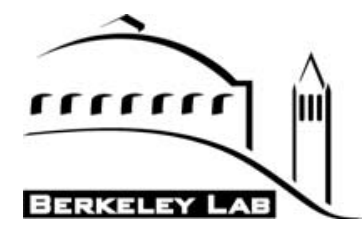

Ernest Orlando Lawrence Berkeley National Laboratory

This work was supported by the Director, Office of Science, U.S. Department of Energy under Contract Number DE- AC02-05CH11231 


\section{Contents}

\section{Volume I}

Preface

Executive Summary …….......................................................................

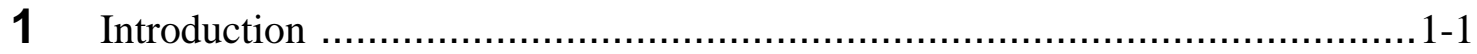

2 Performance-Based Environmental Management System ...........................2-1

3 Environmental Program Summary …………........................................... 3-1

4 Environmental Monitoring ...................................................................

$5 \quad$ Radiological Dose Assessment ........................................................ $5-1$

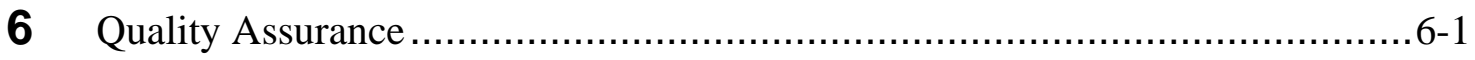

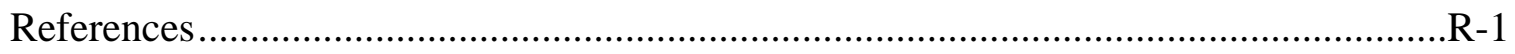

Acronyms and Abbreviations .......................................................................... AA-1

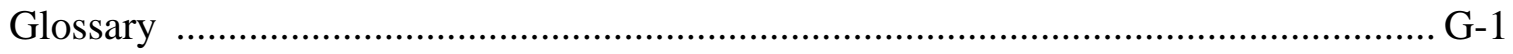

Volume I Distribution List............................................................................ D-1

\section{Volume II}

Appendix $\quad$ Monitoring Data …………………....................................... A-1

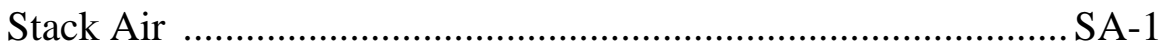

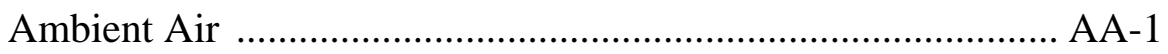

Rainwater ..........................................................................

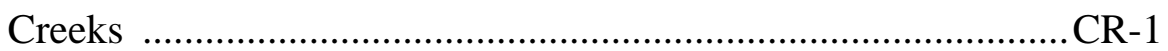

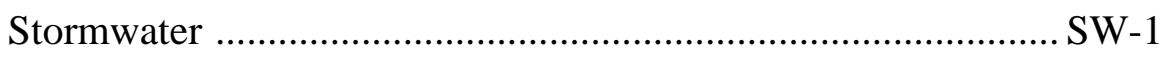

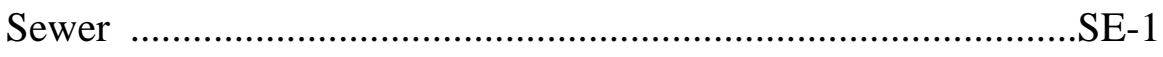

Fixed Treatment Units ................................................................FT-1

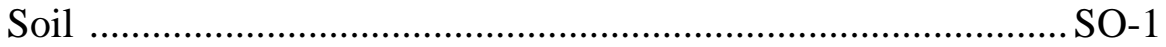

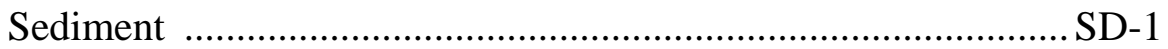




\section{Appendix Monitoring Data}

Volume II of the Site Environmental Report for 2007 is provided by Ernest Orlando Lawrence Berkeley National Laboratory as a supplemental appendix to Volume I, which contains the body of the report. Volume II contains the environmental monitoring and sampling data used to generate summary results of routine and nonroutine activities at the Laboratory (except for groundwater sampling data, which may be found in the reports referred to in Chapter 4). Volume I summarizes the results from analyses of the data.

The results from sample collections are more comprehensive in Volume II than in Volume I: For completeness, all results from sample collections that began or ended in calendar year (CY) 2007 are included in this volume. However, the samples representing CY 2006 data have not been used in the summary results that are reported in Volume I. (For example, although ambient air samples collected on January 9, 2007, are presented in Volume II, they represent December 2006 data and are not included in Table 4-2 in Volume I.)

When appropriate, sampling results are reported in both conventional and International System of Units (SI). For some results, the rounding procedure used in data reporting may result in apparent differences between the numbers reported in SI and conventional units. (For example, stack air tritium results reported as $<1.5 \mathrm{~Bq} / \mathrm{m}^{3}$ are shown variously as $<39$ and $<41 \mathrm{pCi} / \mathrm{m}^{3}$. Both of these results are rounded correctly to two significant digits.)

The list below categorizes the Volume II data sections with corresponding summary result tables in Volume I:

\begin{tabular}{|ll|}
\hline Volume II section & \multicolumn{1}{c|}{ Volume I summary table } \\
\hline Stack Air & No summary table; results discussed in Section 4.2.1 \\
Ambient Air & $\begin{array}{l}\text { 4-2, Summary of Gross Alpha and Gross Beta Ambient-Air Particulate Sampling } \\
\text { Network Results }\end{array}$ \\
Rainwater & No summary table; results discussed in Section 4.3.1.1 \\
Creeks & No summary table; results discussed in Section 4.3.1.2 \\
Stormwater & No summary table; results discussed in Section 4.3.1.3 \\
Sewer & No summary table; results discussed in Section 4.3.2.1.1 - 4.3.2.1.2 \\
Fixed Treatment Units & No summary table; results discussed in Sections 4.3.2.2 - 4.3.2.3 \\
\hline
\end{tabular}




\begin{tabular}{|ll|}
\hline Volume II section & \multicolumn{1}{c|}{ Volume I summary table } \\
\hline Groundwater & No summary table; results discussed in Sections 4.4.1 -4.4 .2 \\
Soil & No summary table; results discussed in Section 4.5.1 \\
Sediment & No summary table; results discussed in Section 4.5 .2 \\
Vegetation & No summary table; results discussed in Section 4.6 \\
\hline
\end{tabular}

The results listed in Volume II identify sampling locations with a station identifier code. The following list cross-references these codes with a more meaningful and descriptive label:

\begin{tabular}{|c|c|c|}
\hline Location code & Description of sampling location & Volume II section \\
\hline 25 FTU & Building 25 fixed treatment unit & Fixed Treatment Units \\
\hline $55-128$ & Building 55, Room 128 & Stack Air \\
\hline 55-128 Backup & $\begin{array}{l}\text { Building 55, Room } 128 \text { inline backup sample (55- } \\
128 \text { Backup results are added to } 55-128 \text { results to } \\
\text { represent total emissions from the location) }\end{array}$ & Stack Air \\
\hline $55-128-\mathrm{COL}$ & $\begin{array}{l}\text { Duplicate sampler collocated with } 55-128 \text { stack air } \\
\text { sampler }\end{array}$ & Stack Air \\
\hline 55-128-COL Backup & $\begin{array}{l}\text { 2nd inline filter at } 55-128-\mathrm{COL} \text { sampler (collocated } \\
\text { with 55-128 stack air sampler) }\end{array}$ & Stack Air \\
\hline 69-Storm Drain & Building 69 storm drain inlet & Stormwater \\
\hline $70-147 A$ & Building 70, Room 147A Berkeley box manifold & Stack Air \\
\hline $70 \mathrm{~A}-1129 \mathrm{H}$ & Building 70A, Room 1129 hood & Stack Air \\
\hline 70A-1129P & Building 70A, Room 1129 pressurized box manifold & Stack Air \\
\hline $75-127-\mathrm{H}$ & Building 75, Room 127 hood & Stack Air \\
\hline 77 FTU & Building 77 fixed treatment unit & Fixed Treatment Units \\
\hline 85 Glovebox & Building 85 (HWHF) penthouse glovebox & Stack Air \\
\hline 85 Hood & Building 85 (HWHF) penthouse hood & Stack Air \\
\hline B88 Cave 0 & Building 88, Cave 0 & Stack Air \\
\hline B88-135H & Building 88, Room 135 hood & Stack Air \\
\hline $\begin{array}{l}\text { Botanical Garden } \\
\text { Creek }\end{array}$ & Botanical Garden Creek & Creeks \\
\hline Building 69 & North side of Building 69 & Soil \\
\hline Building 80 & West side of Building 80 & Soil \\
\hline Building 85 & Northeast of Building 85 & Soil \\
\hline Cafeteria Creek & Cafeteria Creek & Creeks \\
\hline Chicken Creek & Routine sampling at Chicken Creek & $\begin{array}{l}\text { Creeks; Sediment; } \\
\text { Stormwater }\end{array}$ \\
\hline $\begin{array}{l}\text { Chicken Creek- } \\
\text { Downstream }\end{array}$ & $\begin{array}{l}\text { Special site at Chicken Creek for additional } \\
\text { monitoring }\end{array}$ & Creeks \\
\hline
\end{tabular}




\begin{tabular}{|c|c|c|}
\hline Location code & Description of sampling location & Volume II section \\
\hline $\begin{array}{l}\text { Chicken Creek- } \\
\text { Upstream }\end{array}$ & $\begin{array}{l}\text { Special site at Chicken Creek for additional } \\
\text { monitoring }\end{array}$ & Creeks \\
\hline East Canyon & $\begin{array}{l}\text { Between Hazardous Waste Handling Facility and } \\
\text { Centennial Drive }\end{array}$ & Stormwater \\
\hline ENV-44 & North of Building 44 & Ambient Air; Rainwater \\
\hline ENV-44-COL & Duplicate sampler collocated with ENV-44 & Ambient Air \\
\hline ENV-75 & Near Grizzly Peak entrance gate & Rainwater \\
\hline ENV-83 & East of Building 83 & Ambient Air \\
\hline ENV-B13A & Sampling shelter west of Building 88 & Ambient Air \\
\hline ENV-B13C & Background sampling shelter off Panoramic Way & Ambient Air; Soil \\
\hline Field Blank & Blank sample prepared in the field & $\begin{array}{l}\text { Creeks; Fixed Treatment } \\
\text { Units; Rainwater; } \\
\text { Stormwater; Sewer }\end{array}$ \\
\hline Hearst Sewer & Hearst sewer station & Sewer \\
\hline Lot Blank & Blank filter from same lot as submitted samples & Ambient Air, Stack Air \\
\hline $\begin{array}{l}\text { N. Fork Strawberry } \\
\text { Creek }\end{array}$ & $\begin{array}{l}\text { North Fork of Strawberry Creek outlet near western } \\
\text { boundary of site }\end{array}$ & $\begin{array}{l}\text { Creeks; Sediment; } \\
\text { Stormwater }\end{array}$ \\
\hline $\begin{array}{l}\text { N. Fork Strawberry } \\
\text { Creek-Downstream }\end{array}$ & $\begin{array}{l}\text { Special site at North Fork of Strawberry Creek for } \\
\text { additional monitoring }\end{array}$ & Creeks \\
\hline $\begin{array}{l}\text { N. Fork Strawberry } \\
\text { Creek-Upstream }\end{array}$ & $\begin{array}{l}\text { Special site at North Fork of Strawberry Creek for } \\
\text { additional monitoring }\end{array}$ & Creeks \\
\hline NFSC-LBNL & $\begin{array}{l}\text { Special site at North Fork of Strawberry Creek for } \\
\text { Emergency Response Prorotocol for Sewage and } \\
\text { Potable Water Releases }\end{array}$ & Creeks \\
\hline NFSC-LeRoy & $\begin{array}{l}\text { Special site at North Fork of Strawberry Creek for } \\
\text { Emergency Response Prorotocol for Sewage and } \\
\text { Potable Water Releases }\end{array}$ & Creeks \\
\hline NFSC-UCB & $\begin{array}{l}\text { Special site at North Fork of Strawberry Creek for } \\
\text { Emergency Response Prorotocol for Sewage and } \\
\text { Potable Water Releases }\end{array}$ & Creeks \\
\hline No Name Creek & Routine sampling at No Name Creek & Creeks \\
\hline NTLF Hillside Stack & Former NTLF Hillside Stack & Stack Air \\
\hline $\begin{array}{l}\text { NTLF Hillside Stack } \\
\text { Drain }\end{array}$ & Former NTLF Hillside Stack drain line & Stack Air \\
\hline Ravine Creek & Routine sampling at Ravine Creek & Creeks \\
\hline $\begin{array}{l}\text { Strawberry Creek } \\
\text { (UC) }\end{array}$ & Upper Strawberry Creek & Creeks \\
\hline Strawberry Sewer & Strawberry sewer station & Sewer \\
\hline Ten-Inch Creek & Ten-Inch Creek & Creeks \\
\hline Travel Blank & $\begin{array}{l}\text { Blank sample prepared before field collections and } \\
\text { carried by the sample technician during collection } \\
\text { activities }\end{array}$ & Ambient Air, Stack Air \\
\hline
\end{tabular}


The following units are used in Volume II:

\begin{tabular}{|lll|}
\hline Unit & \multicolumn{1}{c|}{ Description } & \multicolumn{1}{c|}{ Pertains to: } \\
\hline$\%$ & Percent & Moisture content of sample \\
$\mu \mathrm{g} / \mathrm{L}$ & Micrograms per liter & Concentration of analyte (nonradioactive) in liquid \\
$\mu \mathrm{mhos} / \mathrm{cm}$ & Micromhos per centimeter & Specific conductance in liquid \\
$\mathrm{Bq} / \mathrm{g}$ & Becquerels per gram & Activity of analyte (radioactive) in solid \\
$\mathrm{Bq} / \mathrm{L}$ & Becquerels per liter & Activity of analyte (radioactive) in liquid \\
$\mathrm{Bq} / \mathrm{m}^{3}$ & Becquerels per cubic meter & Activity of analyte (radioactive) in air \\
$\mathrm{Bq} / \mathrm{S}$ & Becquerels per sample & Activity of analyte (radioactive) in blank samples \\
$\mathrm{mg} / \mathrm{L}$ & Milligrams per liter & Concentration of analyte (nonradioactive) in liquid \\
$\mathrm{MPN} / 100 \mathrm{ml}$ & Most probable number per 100ml & Density of organisms present in liquid \\
$\mathrm{pCi} / \mathrm{g}$ & Picocuries per gram & Activity of analyte (radioactive) in solid \\
$\mathrm{pCi} / \mathrm{L}$ & Picocuries per liter & Activity of analyte (radioactive) in liquid \\
$\mathrm{pCi} / \mathrm{m}^{3}$ & Picocuries per cubic meter & Activity of analyte (radioactive) in air \\
$\mathrm{pCi} / \mathrm{S}$ & Picocuries per sample & Activity of analyte (radioactive) in blank samples \\
$\mathrm{S} . U$. & Standard units & pH measurement \\
\hline
\end{tabular}

\section{Results Below the Detection Limit}

Nonradiological results that cannot be quantified (because they are below the detection limit of the analysis) are reported as less than the reporting limit (for example, “ $<10 \mu \mathrm{g} / \mathrm{L}$ ”). Radiological results that cannot be quantified are generally reported as less than the minimum detectable activity (MDA) (for example, “ $<0.15 \mathrm{~Bq} / \mathrm{L}$ ”). When the MDA is not available, the reporting limit is used. Reporting limits are typically constant between sample results for a particular analyte, but MDAs can vary between sample results for any one analyte. 


\begin{tabular}{|l|c|c|c|c|c|c|}
\hline \multicolumn{1}{|c|}{ Carbon 14 } & \multicolumn{2}{|c|}{ S.I. } & \multicolumn{2}{c|}{ Conventional } & \\
\hline Location & $\begin{array}{c}\text { Collection } \\
\text { Date }\end{array}$ & Result & Units & Result & Units & $\begin{array}{c}\text { QA } \\
\text { Type }\end{array}$ \\
\hline 85 Glovebox & $2 / 6 / 2007$ & $<1.5$ & $\mathrm{~Bq} / \mathrm{m}^{3}$ & $<41$ & $\mathrm{pCi} / \mathrm{m}^{3}$ & Sample \\
\hline & $2 / 6 / 2007$ & $<0.41$ & $\mathrm{~Bq} / \mathrm{m}^{3}$ & $<11$ & $\mathrm{pCi} / \mathrm{m}^{3}$ & Split \\
\hline & $5 / 1 / 2007$ & $<1.5$ & $\mathrm{~Bq} / \mathrm{m}^{3}$ & $<41$ & $\mathrm{pCi} / \mathrm{m}^{3}$ & Sample \\
\hline & $8 / 7 / 2007$ & $<1.5$ & $\mathrm{~Bq} / \mathrm{m}^{3}$ & $<41$ & $\mathrm{pCi} / \mathrm{m}^{3}$ & Sample \\
\hline & $11 / 6 / 2007$ & $<2.7$ & $\mathrm{~Bq} / \mathrm{m}^{3}$ & $<73$ & $\mathrm{pCi} / \mathrm{m}^{3}$ & Sample \\
\hline & $2 / 6 / 2007$ & $<1.5$ & $\mathrm{~Bq} / \mathrm{m}^{3}$ & $<41$ & $\mathrm{pCi} / \mathrm{m}^{3}$ & Sample \\
\hline & $2 / 6 / 2007$ & 0.54 & $\mathrm{~Bq} / \mathrm{m}^{3}$ & 15 & $\mathrm{pCi} / \mathrm{m}^{3}$ & Split \\
\hline & $5 / 8 / 2007$ & $<1.6$ & $\mathrm{~Bq} / \mathrm{m}^{3}$ & $<43$ & $\mathrm{pCi} / \mathrm{m}^{3}$ & Sample \\
\hline & $8 / 7 / 2007$ & $<1.5$ & $\mathrm{~Bq} / \mathrm{m}^{3}$ & $<39$ & $\mathrm{pCi} / \mathrm{m}^{3}$ & Sample \\
\hline & $11 / 6 / 2007$ & $<2.8$ & $\mathrm{~Bq} / \mathrm{m}^{3}$ & $<75$ & $\mathrm{pCi} / \mathrm{m}^{3}$ & Sample \\
\hline & $2 / 6 / 2007$ & $<0.31$ & $\mathrm{~Bq} / \mathrm{S}$ & $<8.5$ & $\mathrm{pCi} / \mathrm{S}$ & Blank \\
\hline TRAVEL BLANK & $2 / 6 / 2007$ & $<2$ & $\mathrm{~Bq} / \mathrm{S}$ & $<54$ & $\mathrm{pCi} / \mathrm{S}$ & Blank \\
\hline & $5 / 1 / 2007$ & $<1.6$ & $\mathrm{~Bq} / \mathrm{S}$ & $<43$ & $\mathrm{pCi} / \mathrm{S}$ & Blank \\
\hline & $8 / 7 / 2007$ & $<1.8$ & $\mathrm{~Bq} / \mathrm{S}$ & $<49$ & $\mathrm{pCi} / \mathrm{S}$ & Blank \\
\hline & $11 / 6 / 2007$ & $<3.7$ & $\mathrm{~Bq} / \mathrm{S}$ & $<100$ & $\mathrm{pCi} / \mathrm{S}$ & Blank \\
\hline
\end{tabular}




\begin{tabular}{|c|c|c|c|c|c|c|}
\hline Gross alpha & & $\mathrm{s}$ & & Conve & ional & \\
\hline Location & $\begin{array}{l}\text { Collection } \\
\text { Date }\end{array}$ & Result & Units & Result & Units & $\begin{array}{l}\text { QA } \\
\text { Type }\end{array}$ \\
\hline $55-128$ & $1 / 2 / 2007$ & $<0.000058$ & $\mathrm{~Bq} / \mathrm{m}^{3}$ & $<0.0016$ & $\mathrm{pCi} / \mathrm{m}^{3}$ & Sample \\
\hline & 2/6/2007 & 0.000069 & $\mathrm{~Bq} / \mathrm{m}^{3}$ & 0.0019 & $\mathrm{pCi} / \mathrm{m}^{3}$ & Sample \\
\hline & 3/7/2007 & $<0.000048$ & $\mathrm{~Bq} / \mathrm{m}^{3}$ & $<0.0013$ & $\mathrm{pCi} / \mathrm{m}^{3}$ & Sample \\
\hline & $4 / 3 / 2007$ & $<0.000047$ & $\mathrm{~Bq} / \mathrm{m}^{3}$ & $<0.0013$ & $\mathrm{pCi} / \mathrm{m}^{3}$ & Sample \\
\hline & $5 / 1 / 2007$ & $<0.000052$ & $\mathrm{~Bq} / \mathrm{m}^{3}$ & $<0.0014$ & $\mathrm{pCi} / \mathrm{m}^{3}$ & Sample \\
\hline & $6 / 5 / 2007$ & $<0.000039$ & $\mathrm{~Bq} / \mathrm{m}^{3}$ & $<0.0011$ & $\mathrm{pCi} / \mathrm{m}^{3}$ & Sample \\
\hline & $7 / 3 / 2007$ & $<0.000051$ & $\mathrm{~Bq} / \mathrm{m}^{3}$ & $<0.0014$ & $\mathrm{pCi} / \mathrm{m}^{3}$ & Sample \\
\hline & $8 / 7 / 2007$ & $<0.000036$ & $\mathrm{~Bq} / \mathrm{m}^{3}$ & $<0.00097$ & $\mathrm{pCi} / \mathrm{m}^{3}$ & Sample \\
\hline & 9/4/2007 & $<0.000048$ & $\mathrm{~Bq} / \mathrm{m}^{3}$ & $<0.0013$ & $\mathrm{pCi} / \mathrm{m}^{3}$ & Sample \\
\hline & $10 / 2 / 2007$ & $<0.000046$ & $\mathrm{~Bq} / \mathrm{m}^{3}$ & $<0.0013$ & $\mathrm{pCi} / \mathrm{m}^{3}$ & Sample \\
\hline & $11 / 6 / 2007$ & $<0.000031$ & $\mathrm{~Bq} / \mathrm{m}^{3}$ & $<0.00084$ & $\mathrm{pCi} / \mathrm{m}^{3}$ & Sample \\
\hline & $12 / 4 / 2007$ & $<0.000053$ & $\mathrm{~Bq} / \mathrm{m}^{3}$ & $<0.0014$ & $\mathrm{pCi} / \mathrm{m}^{3}$ & Sample \\
\hline & $1 / 8 / 2008$ & $<0.00004$ & $\mathrm{~Bq} / \mathrm{m}^{3}$ & $<0.0011$ & $\mathrm{pCi} / \mathrm{m}^{3}$ & Sample \\
\hline $55-128-\mathrm{COL}$ & $1 / 2 / 2007$ & 0.000065 & $\mathrm{~Bq} / \mathrm{m}^{3}$ & 0.0018 & $\mathrm{pCi} / \mathrm{m}^{3}$ & Duplicate \\
\hline & $2 / 6 / 2007$ & 0.000023 & $\mathrm{~Bq} / \mathrm{m}^{3}$ & 0.00062 & $\mathrm{pCi} / \mathrm{m}^{3}$ & Duplicate \\
\hline & 3/7/2007 & 0.000099 & $\mathrm{~Bq} / \mathrm{m}^{3}$ & 0.0027 & $\mathrm{pCi} / \mathrm{m}^{3}$ & Duplicate \\
\hline & $4 / 3 / 2007$ & 0.000068 & $\mathrm{~Bq} / \mathrm{m}^{3}$ & 0.0018 & $\mathrm{pCi} / \mathrm{m}^{3}$ & Duplicate \\
\hline & $5 / 1 / 2007$ & $<0.000021$ & $\mathrm{~Bq} / \mathrm{m}^{3}$ & $<0.00057$ & $\mathrm{pCi} / \mathrm{m}^{3}$ & Duplicate \\
\hline & $6 / 5 / 2007$ & 0.000085 & $\mathrm{~Bq} / \mathrm{m}^{3}$ & 0.0023 & $\mathrm{pCi} / \mathrm{m}^{3}$ & Duplicate \\
\hline & $7 / 3 / 2007$ & 0.000052 & $\mathrm{~Bq} / \mathrm{m}^{3}$ & 0.0014 & $\mathrm{pCi} / \mathrm{m}^{3}$ & Duplicate \\
\hline & 8/7/2007 & $<0.000016$ & $\mathrm{~Bq} / \mathrm{m}^{3}$ & $<0.00042$ & $\mathrm{pCi} / \mathrm{m}^{3}$ & Duplicate \\
\hline & 9/4/2007 & 0.000083 & $\mathrm{~Bq} / \mathrm{m}^{3}$ & 0.0022 & $\mathrm{pCi} / \mathrm{m}^{3}$ & Duplicate \\
\hline & $10 / 2 / 2007$ & $<0.00002$ & $\mathrm{~Bq} / \mathrm{m}^{3}$ & $<0.00054$ & $\mathrm{pCi} / \mathrm{m}^{3}$ & Duplicate \\
\hline & $11 / 6 / 2007$ & $<0.000018$ & $\mathrm{~Bq} / \mathrm{m}^{3}$ & $<0.00049$ & $\mathrm{pCi} / \mathrm{m}^{3}$ & Duplicate \\
\hline & $12 / 4 / 2007$ & 0.000057 & $\mathrm{~Bq} / \mathrm{m}^{3}$ & 0.0015 & $\mathrm{pCi} / \mathrm{m}^{3}$ & Duplicate \\
\hline & $1 / 8 / 2008$ & $<0.000015$ & $\mathrm{~Bq} / \mathrm{m}^{3}$ & $<0.00041$ & $\mathrm{pCi} / \mathrm{m}^{3}$ & Duplicate \\
\hline $70-147 \mathrm{~A}$ & $1 / 2 / 2007$ & $<0.000055$ & $\mathrm{~Bq} / \mathrm{m}^{3}$ & $<0.0015$ & $\mathrm{pCi} / \mathrm{m}^{3}$ & Sample \\
\hline & $2 / 6 / 2007$ & 0.00011 & $\mathrm{~Bq} / \mathrm{m}^{3}$ & 0.003 & $\mathrm{pCi} / \mathrm{m}^{3}$ & Sample \\
\hline & $3 / 6 / 2007$ & $<0.000052$ & $\mathrm{~Bq} / \mathrm{m}^{3}$ & $<0.0014$ & $\mathrm{pCi} / \mathrm{m}^{3}$ & Sample \\
\hline & $4 / 3 / 2007$ & $<0.000045$ & $\mathrm{~Bq} / \mathrm{m}^{3}$ & $<0.0012$ & $\mathrm{pCi} / \mathrm{m}^{3}$ & Sample \\
\hline & $5 / 1 / 2007$ & $<0.000049$ & $\mathrm{~Bq} / \mathrm{m}^{3}$ & $<0.0013$ & $\mathrm{pCi} / \mathrm{m}^{3}$ & Sample \\
\hline
\end{tabular}




\begin{tabular}{|c|c|c|c|c|c|c|}
\hline Gross alpha & & S. & & Conve & ional & \\
\hline Location & $\begin{array}{l}\text { Collection } \\
\text { Date }\end{array}$ & Result & Units & Result & Units & $\begin{array}{c}\text { QA } \\
\text { Type }\end{array}$ \\
\hline $70-147 \mathrm{~A}$ & $6 / 5 / 2007$ & $<0.000037$ & $\mathrm{~Bq} / \mathrm{m}^{3}$ & $<0.001$ & $\mathrm{pCi} / \mathrm{m}^{3}$ & Sample \\
\hline & $7 / 3 / 2007$ & $<0.000046$ & $\mathrm{Bg} / \mathrm{m}^{3}$ & $<0.0012$ & $\mathrm{pCi} / \mathrm{m}^{3}$ & Sample \\
\hline & $8 / 7 / 2007$ & $<0.000034$ & $\mathrm{~Bq} / \mathrm{m}^{3}$ & $<0.00091$ & $\mathrm{pCi} / \mathrm{m}^{3}$ & Sample \\
\hline & 9/4/2007 & $<0.000048$ & $\mathrm{~Bq} / \mathrm{m}^{3}$ & $<0.0013$ & $\mathrm{pCi} / \mathrm{m}^{3}$ & Sample \\
\hline & $10 / 2 / 2007$ & $<0.000045$ & $\mathrm{~Bq} / \mathrm{m}^{3}$ & $<0.0012$ & $\mathrm{pCi} / \mathrm{m}^{3}$ & Sample \\
\hline & $11 / 6 / 2007$ & 0.000032 & $\mathrm{~Bq} / \mathrm{m}^{3}$ & 0.00087 & $\mathrm{pCi} / \mathrm{m}^{3}$ & Sample \\
\hline & $12 / 4 / 2007$ & 0.000045 & $\mathrm{~Bq} / \mathrm{m}^{3}$ & 0.0012 & $\mathrm{pCi} / \mathrm{m}^{3}$ & Sample \\
\hline & $1 / 8 / 2008$ & $<0.000038$ & $\mathrm{~Bq} / \mathrm{m}^{3}$ & $<0.001$ & $\mathrm{pCi} / \mathrm{m}^{3}$ & Sample \\
\hline $70 \mathrm{~A}-1129 \mathrm{H}$ & $1 / 2 / 2007$ & $<0.000055$ & $\mathrm{~Bq} / \mathrm{m}^{3}$ & $<0.0015$ & $\mathrm{pCi} / \mathrm{m}^{3}$ & Sample \\
\hline & 2/6/2007 & 0.000062 & $\mathrm{~Bq} / \mathrm{m}^{3}$ & 0.0017 & $\mathrm{pCi} / \mathrm{m}^{3}$ & Sample \\
\hline & $3 / 6 / 2007$ & $<0.000052$ & $\mathrm{~Bq} / \mathrm{m}^{3}$ & $<0.0014$ & $\mathrm{pCi} / \mathrm{m}^{3}$ & Sample \\
\hline & $4 / 3 / 2007$ & $<0.000044$ & $\mathrm{~Bq} / \mathrm{m}^{3}$ & $<0.0012$ & $\mathrm{pCi} / \mathrm{m}^{3}$ & Sample \\
\hline & $5 / 1 / 2007$ & $<0.000052$ & $\mathrm{~Bq} / \mathrm{m}^{3}$ & $<0.0014$ & $\mathrm{pCi} / \mathrm{m}^{3}$ & Sample \\
\hline & $6 / 5 / 2007$ & $<0.000039$ & $\mathrm{~Bq} / \mathrm{m}^{3}$ & $<0.0011$ & $\mathrm{pCi} / \mathrm{m}^{3}$ & Sample \\
\hline & $7 / 3 / 2007$ & $<0.00005$ & $\mathrm{~Bq} / \mathrm{m}^{3}$ & $<0.0014$ & $\mathrm{pCi} / \mathrm{m}^{3}$ & Sample \\
\hline & 8/7/2007 & $<0.000032$ & $\mathrm{~Bq} / \mathrm{m}^{3}$ & $<0.00087$ & $\mathrm{pCi} / \mathrm{m}^{3}$ & Sample \\
\hline & $9 / 4 / 2007$ & $<0.000046$ & $\mathrm{~Bq} / \mathrm{m}^{3}$ & $<0.0012$ & $\mathrm{pCi} / \mathrm{m}^{3}$ & Sample \\
\hline & $10 / 2 / 2007$ & $<0.000045$ & $\mathrm{~Bq} / \mathrm{m}^{3}$ & $<0.0012$ & $\mathrm{pCi} / \mathrm{m}^{3}$ & Sample \\
\hline & $11 / 6 / 2007$ & $<0.000031$ & $\mathrm{~Bq} / \mathrm{m}^{3}$ & $<0.00082$ & $\mathrm{pCi} / \mathrm{m}^{3}$ & Sample \\
\hline & $12 / 4 / 2007$ & $<0.000046$ & $\mathrm{~Bq} / \mathrm{m}^{3}$ & $<0.0013$ & $\mathrm{pCi} / \mathrm{m}^{3}$ & Sample \\
\hline & $1 / 8 / 2008$ & $<0.000042$ & $\mathrm{~Bq} / \mathrm{m}^{3}$ & $<0.0011$ & $\mathrm{pCi} / \mathrm{m}^{3}$ & Sample \\
\hline $70 \mathrm{~A}-1129 \mathrm{P}$ & $1 / 2 / 2007$ & $<0.000055$ & $\mathrm{~Bq} / \mathrm{m}^{3}$ & $<0.0015$ & $\mathrm{pCi} / \mathrm{m}^{3}$ & Sample \\
\hline & $2 / 6 / 2007$ & 0.000043 & $\mathrm{Bg} / \mathrm{m}^{3}$ & 0.0012 & $\mathrm{pCi} / \mathrm{m}^{3}$ & Sample \\
\hline & $3 / 6 / 2007$ & $<0.000052$ & $\mathrm{~Bq} / \mathrm{m}^{3}$ & $<0.0014$ & $\mathrm{pCi} / \mathrm{m}^{3}$ & Sample \\
\hline & $4 / 3 / 2007$ & $<0.000043$ & $\mathrm{~Bq} / \mathrm{m}^{3}$ & $<0.0012$ & $\mathrm{pCi} / \mathrm{m}^{3}$ & Sample \\
\hline & $5 / 1 / 2007$ & $<0.000051$ & $\mathrm{~Bq} / \mathrm{m}^{3}$ & $<0.0014$ & $\mathrm{pCi} / \mathrm{m}^{3}$ & Sample \\
\hline & $6 / 5 / 2007$ & $<0.000039$ & $\mathrm{~Bq} / \mathrm{m}^{3}$ & $<0.001$ & $\mathrm{pCi} / \mathrm{m}^{3}$ & Sample \\
\hline & $7 / 3 / 2007$ & $<0.000046$ & $\mathrm{~Bq} / \mathrm{m}^{3}$ & $<0.0012$ & $\mathrm{pCi} / \mathrm{m}^{3}$ & Sample \\
\hline & $8 / 7 / 2007$ & $<0.000032$ & $\mathrm{~Bq} / \mathrm{m}^{3}$ & $<0.00086$ & $\mathrm{pCi} / \mathrm{m}^{3}$ & Sample \\
\hline & $9 / 4 / 2007$ & $<0.000046$ & $\mathrm{~Bq} / \mathrm{m}^{3}$ & $<0.0013$ & $\mathrm{pCi} / \mathrm{m}^{3}$ & Sample \\
\hline & $10 / 2 / 2007$ & $<0.000043$ & $\mathrm{~Bq} / \mathrm{m}^{3}$ & $<0.0012$ & $\mathrm{pCi} / \mathrm{m}^{3}$ & Sample \\
\hline & $11 / 6 / 2007$ & 0.000032 & $\mathrm{Bg} / \mathrm{m}^{3}$ & 0.00086 & $\mathrm{pCi} / \mathrm{m}^{3}$ & Sample \\
\hline
\end{tabular}




\begin{tabular}{|c|c|c|c|c|c|c|}
\hline Gross alpha & & \multicolumn{2}{|c|}{ S.I. } & \multicolumn{2}{|c|}{ Conventional } & \multirow[b]{2}{*}{$\begin{array}{c}\text { QA } \\
\text { Type } \\
\end{array}$} \\
\hline Location & $\begin{array}{c}\text { Collection } \\
\text { Date }\end{array}$ & Result & Units & Result & Units & \\
\hline \multirow[t]{2}{*}{$70 A-1129 P$} & $12 / 4 / 2007$ & $<0.000046$ & $\mathrm{~Bq} / \mathrm{m}^{3}$ & $<0.0012$ & $\mathrm{pC} / \mathrm{m}^{3}$ & Sample \\
\hline & $1 / 8 / 2008$ & $<0.000037$ & $\mathrm{Bg} / \mathrm{m}^{3}$ & $<0.001$ & $\mathrm{pCi} / \mathrm{m}^{3}$ & Sample \\
\hline \multirow[t]{6}{*}{ 75-127-H } & 2/6/2007 & 0.000052 & $\mathrm{~Bq} / \mathrm{m}^{3}$ & 0.0014 & $\mathrm{pCi} / \mathrm{m}^{3}$ & Sample \\
\hline & $5 / 1 / 2007$ & $<0.000051$ & $\mathrm{~Bq} / \mathrm{m}^{3}$ & $<0.0014$ & $\mathrm{pCi} / \mathrm{m}^{3}$ & Sample \\
\hline & $8 / 7 / 2007$ & $<0.000033$ & $\mathrm{~Bq} / \mathrm{m}^{3}$ & $<0.00089$ & $\mathrm{pCi} / \mathrm{m}^{3}$ & Sample \\
\hline & $11 / 6 / 2007$ & 0.000039 & $\mathrm{~Bq} / \mathrm{m}^{3}$ & 0.0011 & $\mathrm{pCi} / \mathrm{m}^{3}$ & Sample \\
\hline & $12 / 4 / 2007$ & $<0.000048$ & $\mathrm{~Bq} / \mathrm{m}^{3}$ & $<0.0013$ & $\mathrm{pCi} / \mathrm{m}^{3}$ & Sample \\
\hline & 1/8/2008 & $<0.000038$ & $\mathrm{~Bq} / \mathrm{m}^{3}$ & $<0.001$ & $\mathrm{pCi} / \mathrm{m}^{3}$ & Sample \\
\hline \multirow[t]{5}{*}{85 Glovebox } & 2/6/2007 & 0.000045 & $\mathrm{Bg} / \mathrm{m}^{3}$ & 0.0012 & $\mathrm{pCi} / \mathrm{m}^{3}$ & Sample \\
\hline & $5 / 1 / 2007$ & $<0.000053$ & $\mathrm{~Bq} / \mathrm{m}^{3}$ & $<0.0014$ & $\mathrm{pCi} / \mathrm{m}^{3}$ & Sample \\
\hline & 8/7/2007 & $<0.000034$ & $\mathrm{~Bq} / \mathrm{m}^{3}$ & $<0.00091$ & $\mathrm{pCi} / \mathrm{m}^{3}$ & Sample \\
\hline & 9/4/2007 & $<0.00005$ & $\mathrm{~Bq} / \mathrm{m}^{3}$ & $<0.0013$ & $\mathrm{pCi} / \mathrm{m}^{3}$ & Sample \\
\hline & $11 / 6 / 2007$ & $<0.000035$ & $\mathrm{~Bq} / \mathrm{m}^{3}$ & $<0.00094$ & $\mathrm{pCi} / \mathrm{m}^{3}$ & Sample \\
\hline \multirow[t]{4}{*}{$85 \mathrm{Hood}$} & 2/6/2007 & 0.00003 & $\mathrm{~Bq} / \mathrm{m}^{3}$ & 0.00081 & $\mathrm{pCi} / \mathrm{m}^{3}$ & Sample \\
\hline & $5 / 1 / 2007$ & $<0.000028$ & $\mathrm{Bg} / \mathrm{m}^{3}$ & $<0.00075$ & $\mathrm{pCi} / \mathrm{m}^{3}$ & Sample \\
\hline & $8 / 7 / 2007$ & $<0.000016$ & $\mathrm{~Bq} / \mathrm{m}^{3}$ & $<0.00044$ & $\mathrm{pCi} / \mathrm{m}^{3}$ & Sample \\
\hline & $11 / 6 / 2007$ & $<0.000016$ & $\mathrm{~Bq} / \mathrm{m}^{3}$ & $<0.00043$ & $\mathrm{pCi} / \mathrm{m}^{3}$ & Sample \\
\hline \multirow[t]{4}{*}{ B88 Cave 0} & 2/6/2007 & 0.0001 & $\mathrm{~Bq} / \mathrm{m}^{3}$ & 0.0028 & $\mathrm{pCi} / \mathrm{m}^{3}$ & Sample \\
\hline & $5 / 1 / 2007$ & $<0.000051$ & $\mathrm{Bg} / \mathrm{m}^{3}$ & $<0.0014$ & $\mathrm{pCi} / \mathrm{m}^{3}$ & Sample \\
\hline & 8/7/2007 & $<0.000041$ & $\mathrm{~Bq} / \mathrm{m}^{3}$ & $<0.0011$ & $\mathrm{pCi} / \mathrm{m}^{3}$ & Sample \\
\hline & $11 / 6 / 2007$ & 0.00007 & $\mathrm{~Bq} / \mathrm{m}^{3}$ & 0.0019 & $\mathrm{pCi} / \mathrm{m}^{3}$ & Sample \\
\hline \multirow[t]{4}{*}{ B88-135H } & 2/6/2007 & 0.000038 & $\mathrm{~Bq} / \mathrm{m}^{3}$ & 0.001 & $\mathrm{pCi} / \mathrm{m}^{3}$ & Sample \\
\hline & $5 / 1 / 2007$ & $<0.000051$ & $\mathrm{~Bq} / \mathrm{m}^{3}$ & $<0.0014$ & $\mathrm{pCi} / \mathrm{m}^{3}$ & Sample \\
\hline & 8/7/2007 & $<0.000034$ & $\mathrm{~Bq} / \mathrm{m}^{3}$ & $<0.00092$ & $\mathrm{pCi} / \mathrm{m}^{3}$ & Sample \\
\hline & $11 / 6 / 2007$ & $<0.000033$ & $\mathrm{~Bq} / \mathrm{m}^{3}$ & $<0.00089$ & $\mathrm{pCi} / \mathrm{m}^{3}$ & Sample \\
\hline \multirow[t]{8}{*}{ LOT BLANK } & $1 / 2 / 2007$ & $<0.011$ & $\mathrm{~Bq} / \mathrm{S}$ & $<0.29$ & $\mathrm{pCi} / \mathrm{S}$ & Blank \\
\hline & $1 / 2 / 2007$ & $<0.028$ & $\mathrm{~Bq} / \mathrm{S}$ & $<0.76$ & $\mathrm{pCi} / \mathrm{S}$ & Blank \\
\hline & 2/6/2007 & $<0.011$ & $\mathrm{~Bq} / \mathrm{S}$ & $<0.29$ & $\mathrm{pCi} / \mathrm{S}$ & Blank \\
\hline & $2 / 6 / 2007$ & $<0.013$ & $\mathrm{~Bq} / \mathrm{S}$ & $<0.36$ & $\mathrm{pCi} / \mathrm{S}$ & Blank \\
\hline & $3 / 6 / 2007$ & $<0.026$ & $\mathrm{~Bq} / \mathrm{S}$ & $<0.7$ & $\mathrm{pC}$ Cis & Blank \\
\hline & $3 / 6 / 2007$ & 0.034 & $\mathrm{~Bq} / \mathrm{S}$ & 0.9 & $\mathrm{pCi} / \mathrm{S}$ & Blank \\
\hline & $4 / 3 / 2007$ & $<0.022$ & $\mathrm{~Bq} / \mathrm{S}$ & $<0.6$ & $\mathrm{pCi} / \mathrm{S}$ & Blank \\
\hline & $4 / 3 / 2007$ & 0.032 & $\mathrm{~Bq} / \mathrm{S}$ & 0.86 & $\mathrm{pCi} / \mathrm{S}$ & Blank \\
\hline
\end{tabular}




\begin{tabular}{|c|c|c|c|c|c|c|}
\hline \multirow{2}{*}{$\begin{array}{l}\text { Gross alpha } \\
\text { Location }\end{array}$} & \multirow[b]{2}{*}{$\begin{array}{c}\text { Collection } \\
\text { Date }\end{array}$} & \multicolumn{2}{|c|}{ S.I. } & \multicolumn{2}{|c|}{ Conventional } & \multirow[b]{2}{*}{$\begin{array}{c}\text { QA } \\
\text { Type }\end{array}$} \\
\hline & & Result & Units & Result & Units & \\
\hline \multirow[t]{18}{*}{ LOT BLANK } & $5 / 1 / 2007$ & $<0.027$ & $\mathrm{~Bq} / \mathrm{S}$ & $<0.72$ & $\mathrm{pCi} / \mathrm{S}$ & Blank \\
\hline & $5 / 1 / 2007$ & $<0.012$ & $\mathrm{~Bq} / \mathrm{S}$ & $<0.33$ & $\mathrm{pCi} / \mathrm{S}$ & Blank \\
\hline & $6 / 5 / 2007$ & $<0.024$ & $\mathrm{~Bq} / \mathrm{S}$ & $<0.66$ & $\mathrm{pCi} / \mathrm{S}$ & Blank \\
\hline & $6 / 5 / 2007$ & 0.03 & $\mathrm{~Bq} / \mathrm{S}$ & 0.82 & $\mathrm{pCi} / \mathrm{S}$ & Blank \\
\hline & $7 / 3 / 2007$ & $<0.02$ & $\mathrm{~Bq} / \mathrm{S}$ & $<0.55$ & $\mathrm{pCi} / \mathrm{S}$ & Blank \\
\hline & $7 / 3 / 2007$ & 0.02 & $\mathrm{~Bq} / \mathrm{S}$ & 0.54 & $\mathrm{pCi} / \mathrm{S}$ & Blank \\
\hline & $8 / 7 / 2007$ & $<0.025$ & $\mathrm{~Bq} / \mathrm{S}$ & $<0.68$ & $\mathrm{pCi} / \mathrm{S}$ & Blank \\
\hline & $8 / 7 / 2007$ & $<0.016$ & $\mathrm{~Bq} / \mathrm{S}$ & $<0.42$ & $\mathrm{pCi} / \mathrm{S}$ & Blank \\
\hline & 9/4/2007 & $<0.024$ & $\mathrm{~Bq} / \mathrm{S}$ & $<0.64$ & $\mathrm{pCi} / \mathrm{S}$ & Blank \\
\hline & 9/4/2007 & 0.032 & $\mathrm{~Bq} / \mathrm{S}$ & 0.86 & $\mathrm{pCi} / \mathrm{S}$ & Blank \\
\hline & $10 / 2 / 2007$ & $<0.0087$ & $\mathrm{~Bq} / \mathrm{S}$ & $<0.23$ & $\mathrm{pCi} / \mathrm{S}$ & Blank \\
\hline & $10 / 2 / 2007$ & $<0.023$ & $\mathrm{~Bq} / \mathrm{S}$ & $<0.63$ & $\mathrm{pCi} / \mathrm{S}$ & Blank \\
\hline & $11 / 6 / 2007$ & $<0.013$ & $\mathrm{~Bq} / \mathrm{S}$ & $<0.36$ & $\mathrm{pCi} / \mathrm{S}$ & Blank \\
\hline & $11 / 6 / 2007$ & $<0.02$ & $\mathrm{~Bq} / \mathrm{S}$ & $<0.54$ & $\mathrm{pCi} / \mathrm{S}$ & Blank \\
\hline & $12 / 4 / 2007$ & $<0.023$ & $\mathrm{~Bq} / \mathrm{S}$ & $<0.62$ & $\mathrm{pCi} / \mathrm{S}$ & Blank \\
\hline & $12 / 4 / 2007$ & $<0.01$ & $\mathrm{~Bq} / \mathrm{S}$ & $<0.28$ & $\mathrm{pCi} / \mathrm{S}$ & Blank \\
\hline & $1 / 8 / 2008$ & $<0.0086$ & $\mathrm{~Bq} / \mathrm{S}$ & $<0.23$ & $\mathrm{pCi} / \mathrm{S}$ & Blank \\
\hline & $1 / 8 / 2008$ & $<0.024$ & $\mathrm{~Bq} / \mathrm{S}$ & $<0.64$ & $\mathrm{pCi} / \mathrm{S}$ & Blank \\
\hline \multirow[t]{17}{*}{ TRAVEL BLANK } & $1 / 2 / 2007$ & $<0.028$ & $\mathrm{~Bq} / \mathrm{S}$ & $<0.75$ & $\mathrm{pCi} / \mathrm{S}$ & Blank \\
\hline & 1/2/2007 & $<0.0089$ & $\mathrm{~Bq} / \mathrm{S}$ & $<0.24$ & $\mathrm{pCi} / \mathrm{S}$ & Blank \\
\hline & 2/6/2007 & 0.029 & $\mathrm{~Bq} / \mathrm{S}$ & 0.78 & $\mathrm{pCi} / \mathrm{S}$ & Blank \\
\hline & 2/6/2007 & $<0.011$ & $\mathrm{~Bq} / \mathrm{S}$ & $<0.3$ & $\mathrm{pCi} / \mathrm{S}$ & Blank \\
\hline & $3 / 6 / 2007$ & $<0.027$ & $\mathrm{~Bq} / \mathrm{S}$ & $<0.73$ & $\mathrm{pCi} / \mathrm{S}$ & Blank \\
\hline & $3 / 6 / 2007$ & 0.029 & $\mathrm{~Bq} / \mathrm{S}$ & 0.78 & $\mathrm{pCi} / \mathrm{S}$ & Blank \\
\hline & $4 / 3 / 2007$ & 0.02 & $\mathrm{~Bq} / \mathrm{S}$ & 0.55 & $\mathrm{pCi} / \mathrm{S}$ & Blank \\
\hline & 4/3/2007 & $<0.023$ & $\mathrm{~Bq} / \mathrm{S}$ & $<0.61$ & $\mathrm{pCi} / \mathrm{S}$ & Blank \\
\hline & $5 / 1 / 2007$ & $<0.027$ & $\mathrm{~Bq} / \mathrm{S}$ & $<0.72$ & $\mathrm{pCi} / \mathrm{S}$ & Blank \\
\hline & $5 / 1 / 2007$ & $<0.0087$ & $\mathrm{~Bq} / \mathrm{S}$ & $<0.23$ & $\mathrm{pCi} / \mathrm{S}$ & Blank \\
\hline & $6 / 5 / 2007$ & $<0.025$ & $\mathrm{~Bq} / \mathrm{S}$ & $<0.68$ & $\mathrm{pCi} / \mathrm{S}$ & Blank \\
\hline & $6 / 5 / 2007$ & 0.039 & $\mathrm{~Bq} / \mathrm{S}$ & 1 & $\mathrm{pCi} / \mathrm{S}$ & Blank \\
\hline & $7 / 3 / 2007$ & $<0.021$ & $\mathrm{~Bq} / \mathrm{S}$ & $<0.56$ & $\mathrm{pCi} / \mathrm{S}$ & Blank \\
\hline & $7 / 3 / 2007$ & 0.023 & $\mathrm{~Bq} / \mathrm{S}$ & 0.61 & $\mathrm{pCi} / \mathrm{S}$ & Blank \\
\hline & $8 / 7 / 2007$ & $<0.0091$ & $\mathrm{~Bq} / \mathrm{S}$ & $<0.25$ & $\mathrm{pCi} / \mathrm{S}$ & Blank \\
\hline & $8 / 7 / 2007$ & $<0.021$ & $\mathrm{~Bq} / \mathrm{S}$ & $<0.58$ & $\mathrm{pCi} / \mathrm{S}$ & Blank \\
\hline & $9 / 4 / 2007$ & $<0.024$ & $\mathrm{~Bq} / \mathrm{S}$ & $<0.64$ & $\mathrm{pCi} / \mathrm{S}$ & Blank \\
\hline
\end{tabular}




\begin{tabular}{|l|c|c|c|c|c|c|}
\hline \multicolumn{1}{|c|}{ Gross alpha } & \multicolumn{2}{c|}{ S.I. } & \multicolumn{2}{c|}{ Conventional } & \\
\hline Location & $\begin{array}{c}\text { Collection } \\
\text { Date }\end{array}$ & Result & Units & Result & Units & $\begin{array}{c}\text { QA } \\
\text { Type }\end{array}$ \\
\hline TRAVEL BLANK & $9 / 4 / 2007$ & 0.032 & $\mathrm{~Bq} / \mathrm{S}$ & 0.86 & $\mathrm{pCi} / \mathrm{S}$ & Blank \\
\hline & $10 / 2 / 2007$ & $<0.012$ & $\mathrm{~Bq} / \mathrm{S}$ & $<0.33$ & $\mathrm{pCi} / \mathrm{S}$ & Blank \\
\hline & $10 / 2 / 2007$ & $<0.023$ & $\mathrm{~Bq} / \mathrm{S}$ & $<0.63$ & $\mathrm{pCi} / \mathrm{S}$ & Blank \\
\hline & $11 / 6 / 2007$ & $<0.0097$ & $\mathrm{~Bq} / \mathrm{S}$ & $<0.26$ & $\mathrm{pCi} / \mathrm{S}$ & Blank \\
\hline & $11 / 6 / 2007$ & $<0.021$ & $\mathrm{~Bq} / \mathrm{S}$ & $<0.56$ & $\mathrm{pCi} / \mathrm{S}$ & Blank \\
\hline & $12 / 4 / 2007$ & $<0.026$ & $\mathrm{~Bq} / \mathrm{S}$ & $<0.69$ & $\mathrm{pCi} / \mathrm{S}$ & Blank \\
\hline & $12 / 4 / 2007$ & 0.019 & $\mathrm{~Bq} / \mathrm{S}$ & 0.52 & $\mathrm{pCi} / \mathrm{S}$ & Blank \\
\hline & $1 / 8 / 2008$ & $<0.025$ & $\mathrm{~Bq} / \mathrm{S}$ & $<0.67$ & $\mathrm{pCi} / \mathrm{S}$ & Blank \\
\hline & $1 / 8 / 2008$ & $<0.011$ & $\mathrm{~Bq} / \mathrm{S}$ & $<0.31$ & $\mathrm{pCi} / \mathrm{S}$ & Blank \\
\hline
\end{tabular}




\begin{tabular}{|c|c|c|c|c|c|c|}
\hline Gross beta & & S.I & & Conver & ional & \\
\hline Location & $\begin{array}{c}\text { Collection } \\
\text { Date }\end{array}$ & Result & Units & Result & Units & $\begin{array}{c}\text { QA } \\
\text { Type }\end{array}$ \\
\hline $55-128$ & $1 / 2 / 2007$ & 0.00028 & $\mathrm{~Bq} / \mathrm{m}^{3}$ & 0.0076 & $\mathrm{pCi} / \mathrm{m}^{3}$ & Sample \\
\hline & 2/6/2007 & 0.00051 & $\mathrm{~Bq} / \mathrm{m}^{3}$ & 0.014 & $\mathrm{pCi} / \mathrm{m}^{3}$ & Sample \\
\hline & 3/7/2007 & 0.00019 & $\mathrm{~Bq} / \mathrm{m}^{3}$ & 0.0051 & $\mathrm{pCi} / \mathrm{m}^{3}$ & Sample \\
\hline & 4/3/2007 & 0.00017 & $\mathrm{~Bq} / \mathrm{m}^{3}$ & 0.0046 & $\mathrm{pCi} / \mathrm{m}^{3}$ & Sample \\
\hline & $5 / 1 / 2007$ & 0.00016 & $\mathrm{~Bq} / \mathrm{m}^{3}$ & 0.0044 & $\mathrm{pCi} / \mathrm{m}^{3}$ & Sample \\
\hline & $6 / 5 / 2007$ & 0.0004 & $\mathrm{~Bq} / \mathrm{m}^{3}$ & 0.011 & $\mathrm{pCi} / \mathrm{m}^{3}$ & Sample \\
\hline & $7 / 3 / 2007$ & $<0.000079$ & $\mathrm{~Bq} / \mathrm{m}^{3}$ & $<0.0021$ & $\mathrm{pCi} / \mathrm{m}^{3}$ & Sample \\
\hline & $8 / 7 / 2007$ & 0.00031 & $\mathrm{Bg} / \mathrm{m}^{3}$ & 0.0084 & $\mathrm{pCi} / \mathrm{m}^{3}$ & Sample \\
\hline & 9/4/2007 & 0.00057 & $\mathrm{~Bq} / \mathrm{m}^{3}$ & 0.015 & $\mathrm{pCi} / \mathrm{m}^{3}$ & Sample \\
\hline & $10 / 2 / 2007$ & 0.0005 & $\mathrm{~Bq} / \mathrm{m}^{3}$ & 0.014 & $\mathrm{pCi} / \mathrm{m}^{3}$ & Sample \\
\hline & $11 / 6 / 2007$ & 0.00029 & $\mathrm{~Bq} / \mathrm{m}^{3}$ & 0.0079 & $\mathrm{pCi} / \mathrm{m}^{3}$ & Sample \\
\hline & $12 / 4 / 2007$ & 0.0004 & $\mathrm{~Bq} / \mathrm{m}^{3}$ & 0.011 & $\mathrm{pCi} / \mathrm{m}^{3}$ & Sample \\
\hline & $1 / 8 / 2008$ & 0.00018 & $\mathrm{~Bq} / \mathrm{m}^{3}$ & 0.0049 & $\mathrm{pCi} / \mathrm{m}^{3}$ & Sample \\
\hline $55-128-\mathrm{COL}$ & $1 / 2 / 2007$ & 0.00032 & $\mathrm{~Bq} / \mathrm{m}^{3}$ & 0.0087 & $\mathrm{pCi} / \mathrm{m}^{3}$ & Duplicate \\
\hline & 2/6/2007 & 0.00047 & $\mathrm{Bg} / \mathrm{m}^{3}$ & 0.013 & $\mathrm{pCi} / \mathrm{m}^{3}$ & Duplicate \\
\hline & 3/7/2007 & 0.0002 & $\mathrm{~Bq} / \mathrm{m}^{3}$ & 0.0055 & $\mathrm{pCi} / \mathrm{m}^{3}$ & Duplicate \\
\hline & $4 / 3 / 2007$ & 0.000092 & $\mathrm{~Bq} / \mathrm{m}^{3}$ & 0.0025 & $\mathrm{pCi} / \mathrm{m}^{3}$ & Duplicate \\
\hline & $5 / 1 / 2007$ & 0.00013 & $\mathrm{~Bq} / \mathrm{m}^{3}$ & 0.0035 & $\mathrm{pCi} / \mathrm{m}^{3}$ & Duplicate \\
\hline & $6 / 5 / 2007$ & 0.00016 & $\mathrm{~Bq} / \mathrm{m}^{3}$ & 0.0043 & $\mathrm{pCi} / \mathrm{m}^{3}$ & Duplicate \\
\hline & $7 / 3 / 2007$ & 0.000066 & $\mathrm{~Bq} / \mathrm{m}^{3}$ & 0.0018 & $\mathrm{pCi} / \mathrm{m}^{3}$ & Duplicate \\
\hline & 8/7/2007 & 0.00012 & $\mathrm{~Bq} / \mathrm{m}^{3}$ & 0.0033 & $\mathrm{pCi} / \mathrm{m}^{3}$ & Duplicate \\
\hline & $9 / 4 / 2007$ & 0.00053 & $\mathrm{~Bq} / \mathrm{m}^{3}$ & 0.014 & $\mathrm{pCi} / \mathrm{m}^{3}$ & Duplicate \\
\hline & $10 / 2 / 2007$ & 0.00039 & $\mathrm{~Bq} / \mathrm{m}^{3}$ & 0.01 & $\mathrm{pCi} / \mathrm{m}^{3}$ & Duplicate \\
\hline & $11 / 6 / 2007$ & 0.00033 & $\mathrm{~Bq} / \mathrm{m}^{3}$ & 0.009 & $\mathrm{pCi} / \mathrm{m}^{3}$ & Duplicate \\
\hline & $12 / 4 / 2007$ & 0.00022 & $\mathrm{~Bq} / \mathrm{m}^{3}$ & 0.0058 & $\mathrm{pCi} / \mathrm{m}^{3}$ & Duplicate \\
\hline & $1 / 8 / 2008$ & 0.00016 & $\mathrm{~Bq} / \mathrm{m}^{3}$ & 0.0043 & $\mathrm{pCi} / \mathrm{m}^{3}$ & Duplicate \\
\hline 70-147A & $1 / 2 / 2007$ & 0.00038 & $\mathrm{~Bq} / \mathrm{m}^{3}$ & 0.01 & $\mathrm{pCi} / \mathrm{m}^{3}$ & Sample \\
\hline & 2/6/2007 & 0.00087 & $\mathrm{~Bq} / \mathrm{m}^{3}$ & 0.024 & $\mathrm{pCi} / \mathrm{m}^{3}$ & Sample \\
\hline & $3 / 6 / 2007$ & 0.00019 & $\mathrm{~Bq} / \mathrm{m}^{3}$ & 0.0052 & $\mathrm{pCi} / \mathrm{m}^{3}$ & Sample \\
\hline & 4/3/2007 & 0.00035 & $\mathrm{~Bq} / \mathrm{m}^{3}$ & 0.0095 & $\mathrm{pCi} / \mathrm{m}^{3}$ & Sample \\
\hline & $5 / 1 / 2007$ & 0.00015 & $\mathrm{Bg} / \mathrm{m}^{3}$ & 0.0041 & $\mathrm{pCi} / \mathrm{m}^{3}$ & Sample \\
\hline
\end{tabular}




\begin{tabular}{|c|c|c|c|c|c|c|}
\hline Gross beta & & S. & & Conve & onal & \\
\hline Location & $\begin{array}{c}\text { Collection } \\
\text { Date }\end{array}$ & Result & Units & Result & Units & $\begin{array}{c}\text { QA } \\
\text { Type }\end{array}$ \\
\hline $70-147 \mathrm{~A}$ & $6 / 5 / 2007$ & 0.00018 & $\mathrm{~Bq} / \mathrm{m}^{3}$ & 0.0048 & $\mathrm{pCi} / \mathrm{m}^{3}$ & Sample \\
\hline & $7 / 3 / 2007$ & 0.00011 & $\mathrm{Bg} / \mathrm{m}^{3}$ & 0.0029 & $\mathrm{pCi} / \mathrm{m}^{3}$ & Sample \\
\hline & $8 / 7 / 2007$ & 0.000076 & $\mathrm{~Bq} / \mathrm{m}^{3}$ & 0.0021 & $\mathrm{pCi} / \mathrm{m}^{3}$ & Sample \\
\hline & 9/4/2007 & 0.0001 & $\mathrm{~Bq} / \mathrm{m}^{3}$ & 0.0027 & $\mathrm{pCi} / \mathrm{m}^{3}$ & Sample \\
\hline & $10 / 2 / 2007$ & 0.00023 & $\mathrm{~Bq} / \mathrm{m}^{3}$ & 0.0063 & $\mathrm{pCi} / \mathrm{m}^{3}$ & Sample \\
\hline & $11 / 6 / 2007$ & 0.00029 & $\mathrm{~Bq} / \mathrm{m}^{3}$ & 0.0079 & $\mathrm{pCi} / \mathrm{m}^{3}$ & Sample \\
\hline & $12 / 4 / 2007$ & 0.00049 & $\mathrm{~Bq} / \mathrm{m}^{3}$ & 0.013 & $\mathrm{pCi} / \mathrm{m}^{3}$ & Sample \\
\hline & $1 / 8 / 2008$ & 0.00018 & $\mathrm{~Bq} / \mathrm{m}^{3}$ & 0.0048 & $\mathrm{pCi} / \mathrm{m}^{3}$ & Sample \\
\hline $70 \mathrm{~A}-1129 \mathrm{H}$ & $1 / 2 / 2007$ & 0.00013 & $\mathrm{~Bq} / \mathrm{m}^{3}$ & 0.0034 & $\mathrm{pCi} / \mathrm{m}^{3}$ & Sample \\
\hline & 2/6/2007 & 0.00017 & $\mathrm{~Bq} / \mathrm{m}^{3}$ & 0.0047 & $\mathrm{pCi} / \mathrm{m}^{3}$ & Sample \\
\hline & $3 / 6 / 2007$ & $<0.000069$ & $\mathrm{~Bq} / \mathrm{m}^{3}$ & $<0.0019$ & $\mathrm{pCi} / \mathrm{m}^{3}$ & Sample \\
\hline & $4 / 3 / 2007$ & 0.0001 & $\mathrm{~Bq} / \mathrm{m}^{3}$ & 0.0028 & $\mathrm{pCi} / \mathrm{m}^{3}$ & Sample \\
\hline & $5 / 1 / 2007$ & 0.000071 & $\mathrm{~Bq} / \mathrm{m}^{3}$ & 0.0019 & $\mathrm{pCi} / \mathrm{m}^{3}$ & Sample \\
\hline & $6 / 5 / 2007$ & 0.000081 & $\mathrm{~Bq} / \mathrm{m}^{3}$ & 0.0022 & $\mathrm{pCi} / \mathrm{m}^{3}$ & Sample \\
\hline & $7 / 3 / 2007$ & $<0.000076$ & $\mathrm{~Bq} / \mathrm{m}^{3}$ & $<0.002$ & $\mathrm{pCi} / \mathrm{m}^{3}$ & Sample \\
\hline & $8 / 7 / 2007$ & $<0.000056$ & $\mathrm{~Bq} / \mathrm{m}^{3}$ & $<0.0015$ & $\mathrm{pCi} / \mathrm{m}^{3}$ & Sample \\
\hline & 9/4/2007 & $<0.000078$ & $\mathrm{~Bq} / \mathrm{m}^{3}$ & $<0.0021$ & $\mathrm{pCi} / \mathrm{m}^{3}$ & Sample \\
\hline & $10 / 2 / 2007$ & 0.00009 & $\mathrm{~Bq} / \mathrm{m}^{3}$ & 0.0024 & $\mathrm{pCi} / \mathrm{m}^{3}$ & Sample \\
\hline & $11 / 6 / 2007$ & 0.00023 & $\mathrm{~Bq} / \mathrm{m}^{3}$ & 0.0061 & $\mathrm{pCi} / \mathrm{m}^{3}$ & Sample \\
\hline & $12 / 4 / 2007$ & 0.00017 & $\mathrm{~Bq} / \mathrm{m}^{3}$ & 0.0045 & $\mathrm{pCi} / \mathrm{m}^{3}$ & Sample \\
\hline & $1 / 8 / 2008$ & 0.000071 & $\mathrm{~Bq} / \mathrm{m}^{3}$ & 0.0019 & $\mathrm{pCi} / \mathrm{m}^{3}$ & Sample \\
\hline $70 \mathrm{~A}-1129 \mathrm{P}$ & $1 / 2 / 2007$ & $<0.000072$ & $\mathrm{~Bq} / \mathrm{m}^{3}$ & $<0.0019$ & $\mathrm{pCi} / \mathrm{m}^{3}$ & Sample \\
\hline & $2 / 6 / 2007$ & 0.00012 & $\mathrm{Bg} / \mathrm{m}^{3}$ & 0.0031 & $\mathrm{pCi} / \mathrm{m}^{3}$ & Sample \\
\hline & $3 / 6 / 2007$ & $<0.000069$ & $\mathrm{~Bq} / \mathrm{m}^{3}$ & $<0.0019$ & $\mathrm{pCi} / \mathrm{m}^{3}$ & Sample \\
\hline & $4 / 3 / 2007$ & 0.00011 & $\mathrm{~Bq} / \mathrm{m}^{3}$ & 0.0029 & $\mathrm{pCi} / \mathrm{m}^{3}$ & Sample \\
\hline & $5 / 1 / 2007$ & $<0.000063$ & $\mathrm{~Bq} / \mathrm{m}^{3}$ & $<0.0017$ & $\mathrm{pCi} / \mathrm{m}^{3}$ & Sample \\
\hline & $6 / 5 / 2007$ & $<0.000052$ & $\mathrm{~Bq} / \mathrm{m}^{3}$ & $<0.0014$ & $\mathrm{pCi} / \mathrm{m}^{3}$ & Sample \\
\hline & $7 / 3 / 2007$ & $<0.000072$ & $\mathrm{~Bq} / \mathrm{m}^{3}$ & $<0.0019$ & $\mathrm{pCi} / \mathrm{m}^{3}$ & Sample \\
\hline & $8 / 7 / 2007$ & $<0.000056$ & $\mathrm{~Bq} / \mathrm{m}^{3}$ & $<0.0015$ & $\mathrm{pCi} / \mathrm{m}^{3}$ & Sample \\
\hline & 9/4/2007 & $<0.000079$ & $\mathrm{~Bq} / \mathrm{m}^{3}$ & $<0.0021$ & $\mathrm{pCi} / \mathrm{m}^{3}$ & Sample \\
\hline & $10 / 2 / 2007$ & $<0.000077$ & $\mathrm{~Bq} / \mathrm{m}^{3}$ & $<0.0021$ & $\mathrm{pCi} / \mathrm{m}^{3}$ & Sample \\
\hline & $11 / 6 / 2007$ & 0.000088 & $\mathrm{Bg} / \mathrm{m}^{3}$ & 0.0024 & $\mathrm{pCi} / \mathrm{m}^{3}$ & Sample \\
\hline
\end{tabular}




\begin{tabular}{|c|c|c|c|c|c|c|}
\hline Gross beta & & S.I & & Conve & ional & \\
\hline Location & $\begin{array}{c}\text { Collection } \\
\text { Date }\end{array}$ & Result & Units & Result & Units & $\begin{array}{c}\text { QA } \\
\text { Type }\end{array}$ \\
\hline $70 \mathrm{~A}-1129 \mathrm{P}$ & $12 / 4 / 2007$ & 0.00008 & $\mathrm{~Bq} / \mathrm{m}^{3}$ & 0.0021 & $\mathrm{pCi} / \mathrm{m}^{3}$ & Sample \\
\hline & 1/8/2008 & $<0.000054$ & $\mathrm{~Bq} / \mathrm{m}^{3}$ & $<0.0015$ & $\mathrm{pCi} / \mathrm{m}^{3}$ & Sample \\
\hline $75-127-\mathrm{H}$ & 2/6/2007 & $<0.000056$ & $\mathrm{~Bq} / \mathrm{m}^{3}$ & $<0.0015$ & $\mathrm{pCi} / \mathrm{m}^{3}$ & Sample \\
\hline & $5 / 1 / 2007$ & $<0.000063$ & $\mathrm{~Bq} / \mathrm{m}^{3}$ & $<0.0017$ & $\mathrm{pCi} / \mathrm{m}^{3}$ & Sample \\
\hline & 8/7/2007 & $<0.000055$ & $\mathrm{~Bq} / \mathrm{m}^{3}$ & $<0.0015$ & $\mathrm{pCi} / \mathrm{m}^{3}$ & Sample \\
\hline & $11 / 6 / 2007$ & 0.00035 & $\mathrm{~Bq} / \mathrm{m}^{3}$ & 0.0094 & $\mathrm{pCi} / \mathrm{m}^{3}$ & Sample \\
\hline & $12 / 4 / 2007$ & 0.00038 & $\mathrm{~Bq} / \mathrm{m}^{3}$ & 0.01 & $\mathrm{pCi} / \mathrm{m}^{3}$ & Sample \\
\hline & $1 / 8 / 2008$ & 0.00021 & $\mathrm{~Bq} / \mathrm{m}^{3}$ & 0.0058 & $\mathrm{pCi} / \mathrm{m}^{3}$ & Sample \\
\hline 85 Glovebox & 2/6/2007 & $<0.000058$ & $\mathrm{~Bq} / \mathrm{m}^{3}$ & $<0.0016$ & $\mathrm{pCi} / \mathrm{m}^{3}$ & Sample \\
\hline & $5 / 1 / 2007$ & $<0.000064$ & $\mathrm{~Bq} / \mathrm{m}^{3}$ & $<0.0017$ & $\mathrm{pCi} / \mathrm{m}^{3}$ & Sample \\
\hline & $8 / 7 / 2007$ & $<0.000055$ & $\mathrm{~Bq} / \mathrm{m}^{3}$ & $<0.0015$ & $\mathrm{pCi} / \mathrm{m}^{3}$ & Sample \\
\hline & 9/4/2007 & $<0.000083$ & $\mathrm{~Bq} / \mathrm{m}^{3}$ & $<0.0023$ & $\mathrm{pCi} / \mathrm{m}^{3}$ & Sample \\
\hline & $11 / 6 / 2007$ & $<0.000051$ & $\mathrm{~Bq} / \mathrm{m}^{3}$ & $<0.0014$ & $\mathrm{pCi} / \mathrm{m}^{3}$ & Sample \\
\hline $85 \mathrm{Hood}$ & 2/6/2007 & 0.000061 & $\mathrm{~Bq} / \mathrm{m}^{3}$ & 0.0016 & $\mathrm{pCi} / \mathrm{m}^{3}$ & Sample \\
\hline & $5 / 1 / 2007$ & $<0.000034$ & $\mathrm{Bg} / \mathrm{m}^{3}$ & $<0.00091$ & $\mathrm{pCi} / \mathrm{m}^{3}$ & Sample \\
\hline & $8 / 7 / 2007$ & $<0.000028$ & $\mathrm{~Bq} / \mathrm{m}^{3}$ & $<0.00075$ & $\mathrm{pCi} / \mathrm{m}^{3}$ & Sample \\
\hline & $11 / 6 / 2007$ & 0.000044 & $\mathrm{~Bq} / \mathrm{m}^{3}$ & 0.0012 & $\mathrm{pCi} / \mathrm{m}^{3}$ & Sample \\
\hline B88 Cave 0 & $2 / 6 / 2007$ & 0.00094 & $\mathrm{~Bq} / \mathrm{m}^{3}$ & 0.025 & $\mathrm{pCi} / \mathrm{m}^{3}$ & Sample \\
\hline & $5 / 1 / 2007$ & $<0.000065$ & $\mathrm{~Bq} / \mathrm{m}^{3}$ & $<0.0017$ & $\mathrm{pCi} / \mathrm{m}^{3}$ & Sample \\
\hline & $8 / 7 / 2007$ & 0.00008 & $\mathrm{~Bq} / \mathrm{m}^{3}$ & 0.0022 & $\mathrm{pCi} / \mathrm{m}^{3}$ & Sample \\
\hline & $11 / 6 / 2007$ & 0.00056 & $\mathrm{~Bq} / \mathrm{m}^{3}$ & 0.015 & $\mathrm{pCi} / \mathrm{m}^{3}$ & Sample \\
\hline B88-135H & 2/6/2007 & 0.000099 & $\mathrm{~Bq} / \mathrm{m}^{3}$ & 0.0027 & $\mathrm{pCi} / \mathrm{m}^{3}$ & Sample \\
\hline & $5 / 1 / 2007$ & $<0.000067$ & $\mathrm{~Bq} / \mathrm{m}^{3}$ & $<0.0018$ & $\mathrm{pCi} / \mathrm{m}^{3}$ & Sample \\
\hline & $8 / 7 / 2007$ & $<0.000055$ & $\mathrm{Bg} / \mathrm{m}^{3}$ & $<0.0015$ & $\mathrm{pCi} / \mathrm{m}^{3}$ & Sample \\
\hline & $11 / 6 / 2007$ & 0.000083 & $\mathrm{~Bq} / \mathrm{m}^{3}$ & 0.0023 & $\mathrm{pCi} / \mathrm{m}^{3}$ & Sample \\
\hline LOT BLANK & $1 / 2 / 2007$ & $<0.021$ & $\mathrm{~Bq} / \mathrm{S}$ & $<0.57$ & $\mathrm{pCi} / \mathrm{S}$ & Blank \\
\hline & $1 / 2 / 2007$ & $<0.038$ & $\mathrm{~Bq} / \mathrm{S}$ & $<1$ & $\mathrm{pCi} / \mathrm{S}$ & Blank \\
\hline & 2/6/2007 & $<0.035$ & $\mathrm{~Bq} / \mathrm{S}$ & $<0.94$ & $\mathrm{pCi} / \mathrm{S}$ & Blank \\
\hline & 2/6/2007 & $<0.022$ & $\mathrm{~Bq} / \mathrm{S}$ & $<0.6$ & $\mathrm{pCi} / \mathrm{S}$ & Blank \\
\hline & $3 / 6 / 2007$ & $<0.035$ & $\mathrm{~Bq} / \mathrm{S}$ & $<0.94$ & $\mathrm{pCi} / \mathrm{S}$ & Blank \\
\hline & $3 / 6 / 2007$ & $<0.018$ & $\mathrm{~Bq} / \mathrm{S}$ & $<0.48$ & $\mathrm{pCi} / \mathrm{S}$ & Blank \\
\hline & $4 / 3 / 2007$ & $<0.021$ & $\mathrm{~Bq} / \mathrm{S}$ & $<0.57$ & $\mathrm{pCi} / \mathrm{S}$ & Blank \\
\hline & 4/3/2007 & $<0.031$ & $\mathrm{~Bq} / \mathrm{S}$ & $<0.84$ & $\mathrm{pCi} / \mathrm{S}$ & Blank \\
\hline
\end{tabular}




\begin{tabular}{|c|c|c|c|c|c|c|}
\hline Gross beta & & S. & & Conve & onal & \\
\hline Location & $\begin{array}{c}\text { Collection } \\
\text { Date }\end{array}$ & Result & Units & Result & Units & $\begin{array}{l}\text { QA } \\
\text { Type }\end{array}$ \\
\hline LOT BLANK & $5 / 1 / 2007$ & $<0.032$ & $\mathrm{~Bq} / \mathrm{S}$ & $<0.87$ & $\mathrm{pCi} / \mathrm{S}$ & Blank \\
\hline & $5 / 1 / 2007$ & $<0.023$ & $\mathrm{~Bq} / \mathrm{S}$ & $<0.63$ & $\mathrm{pCi} / \mathrm{S}$ & Blank \\
\hline & $6 / 5 / 2007$ & $<0.033$ & $\mathrm{~Bq} / \mathrm{S}$ & $<0.89$ & $\mathrm{pCi} / \mathrm{S}$ & Blank \\
\hline & $6 / 5 / 2007$ & $<0.017$ & $\mathrm{~Bq} / \mathrm{S}$ & $<0.45$ & $\mathrm{pCi} / \mathrm{S}$ & Blank \\
\hline & $7 / 3 / 2007$ & $<0.036$ & $\mathrm{~Bq} / \mathrm{S}$ & $<0.98$ & $\mathrm{pCi} / \mathrm{S}$ & Blank \\
\hline & $7 / 3 / 2007$ & $<0.025$ & $\mathrm{~Bq} / \mathrm{S}$ & $<0.68$ & $\mathrm{pCi} / \mathrm{S}$ & Blank \\
\hline & $8 / 7 / 2007$ & $<0.038$ & $\mathrm{~Bq} / \mathrm{S}$ & $<1$ & $\mathrm{pCi} / \mathrm{S}$ & Blank \\
\hline & $8 / 7 / 2007$ & $<0.022$ & $\mathrm{~Bq} / \mathrm{S}$ & $<0.6$ & $\mathrm{pCi} / \mathrm{S}$ & Blank \\
\hline & 9/4/2007 & 0.033 & $\mathrm{~Bq} / \mathrm{S}$ & 0.88 & $\mathrm{pCi} / \mathrm{S}$ & Blank \\
\hline & 9/4/2007 & $<0.04$ & $\mathrm{~Bq} / \mathrm{S}$ & $<1.1$ & $\mathrm{pCi} / \mathrm{S}$ & Blank \\
\hline & $10 / 2 / 2007$ & $<0.04$ & $\mathrm{~Bq} / \mathrm{S}$ & $<1.1$ & $\mathrm{pCi} / \mathrm{S}$ & Blank \\
\hline & $10 / 2 / 2007$ & $<0.022$ & $\mathrm{~Bq} / \mathrm{S}$ & $<0.58$ & $\mathrm{pCi} / \mathrm{S}$ & Blank \\
\hline & $11 / 6 / 2007$ & $<0.031$ & $\mathrm{~Bq} / \mathrm{S}$ & $<0.85$ & $\mathrm{pCi} / \mathrm{S}$ & Blank \\
\hline & $11 / 6 / 2007$ & 0.027 & $\mathrm{~Bq} / \mathrm{S}$ & 0.73 & $\mathrm{pCi} / \mathrm{S}$ & Blank \\
\hline & $12 / 4 / 2007$ & $<0.033$ & $\mathrm{~Bq} / \mathrm{S}$ & $<0.89$ & $\mathrm{pCi} / \mathrm{S}$ & Blank \\
\hline & $12 / 4 / 2007$ & 0.021 & $\mathrm{~Bq} / \mathrm{S}$ & 0.55 & $\mathrm{pCi} / \mathrm{S}$ & Blank \\
\hline & $1 / 8 / 2008$ & $<0.021$ & $\mathrm{~Bq} / \mathrm{S}$ & $<0.56$ & $\mathrm{pCi} / \mathrm{S}$ & Blank \\
\hline & $1 / 8 / 2008$ & $<0.035$ & $\mathrm{~Bq} / \mathrm{S}$ & $<0.94$ & $\mathrm{pCi} / \mathrm{S}$ & Blank \\
\hline TRAVEL BLANK & $1 / 2 / 2007$ & $<0.019$ & $\mathrm{~Bq} / \mathrm{S}$ & $<0.51$ & $\mathrm{pCi} / \mathrm{S}$ & Blank \\
\hline & $1 / 2 / 2007$ & $<0.037$ & $\mathrm{~Bq} / \mathrm{S}$ & $<0.99$ & $\mathrm{pCi} / \mathrm{S}$ & Blank \\
\hline & $2 / 6 / 2007$ & $<0.036$ & $\mathrm{~Bq} / \mathrm{S}$ & $<0.96$ & $\mathrm{pCi} / \mathrm{S}$ & Blank \\
\hline & 2/6/2007 & $<0.023$ & $\mathrm{~Bq} / \mathrm{S}$ & $<0.63$ & $\mathrm{pCi} / \mathrm{S}$ & Blank \\
\hline & $3 / 6 / 2007$ & $<0.016$ & $\mathrm{~Bq} / \mathrm{S}$ & $<0.43$ & $\mathrm{pCi} / \mathrm{S}$ & Blank \\
\hline & $3 / 6 / 2007$ & $<0.035$ & $\mathrm{~Bq} / \mathrm{S}$ & $<0.95$ & $\mathrm{pCi} / \mathrm{S}$ & Blank \\
\hline & $4 / 3 / 2007$ & $<0.021$ & $\mathrm{~Bq} / \mathrm{S}$ & $<0.57$ & $\mathrm{pCi} / \mathrm{S}$ & Blank \\
\hline & $4 / 3 / 2007$ & $<0.032$ & $\mathrm{~Bq} / \mathrm{S}$ & $<0.86$ & $\mathrm{pCi} / \mathrm{S}$ & Blank \\
\hline & $5 / 1 / 2007$ & $<0.023$ & $\mathrm{~Bq} / \mathrm{S}$ & $<0.62$ & $\mathrm{pCi} / \mathrm{S}$ & Blank \\
\hline & $5 / 1 / 2007$ & $<0.032$ & $\mathrm{~Bq} / \mathrm{S}$ & $<0.87$ & $\mathrm{pCi} / \mathrm{S}$ & Blank \\
\hline & $6 / 5 / 2007$ & $<0.023$ & $\mathrm{~Bq} / \mathrm{S}$ & $<0.63$ & $\mathrm{pCi} / \mathrm{S}$ & Blank \\
\hline & $6 / 5 / 2007$ & $<0.033$ & $\mathrm{~Bq} / \mathrm{S}$ & $<0.9$ & $\mathrm{pCi} / \mathrm{S}$ & Blank \\
\hline & $7 / 3 / 2007$ & $<0.028$ & $\mathrm{~Bq} / \mathrm{S}$ & $<0.75$ & $\mathrm{pCi} / \mathrm{S}$ & Blank \\
\hline & $7 / 3 / 2007$ & $<0.035$ & $\mathrm{~Bq} / \mathrm{S}$ & $<0.95$ & $\mathrm{pCi} / \mathrm{S}$ & Blank \\
\hline & $8 / 7 / 2007$ & $<0.021$ & $\mathrm{~Bq} / \mathrm{S}$ & $<0.56$ & $\mathrm{pCi} / \mathrm{S}$ & Blank \\
\hline & $8 / 7 / 2007$ & $<0.034$ & $\mathrm{~Bq} / \mathrm{S}$ & $<0.93$ & $\mathrm{pCi} / \mathrm{S}$ & Blank \\
\hline & 9/4/2007 & 0.039 & $\mathrm{~Bq} / \mathrm{S}$ & 1 & $\mathrm{pCi} / \mathrm{S}$ & Blank \\
\hline
\end{tabular}




\begin{tabular}{|l|c|c|c|r|r|c|}
\hline \multicolumn{1}{|c|}{ Gross beta } & \multicolumn{2}{|c|}{ S.I. } & \multicolumn{2}{c|}{ Conventional } & \\
\hline Location & $\begin{array}{c}\text { Collection } \\
\text { Date }\end{array}$ & Result & Units & Result & Units & $\begin{array}{c}\text { QA } \\
\text { Type }\end{array}$ \\
\hline TRAVEL BLANK & $9 / 4 / 2007$ & $<0.038$ & $\mathrm{~Bq} / \mathrm{S}$ & $<1$ & $\mathrm{pCi} / \mathrm{S}$ & Blank \\
\hline & $10 / 2 / 2007$ & $<0.021$ & $\mathrm{~Bq} / \mathrm{S}$ & $<0.56$ & $\mathrm{pCi} / \mathrm{S}$ & Blank \\
\hline & $10 / 2 / 2007$ & $<0.037$ & $\mathrm{~Bq} / \mathrm{S}$ & $<1$ & $\mathrm{pCi} / \mathrm{S}$ & Blank \\
\hline & $11 / 6 / 2007$ & 0.026 & $\mathrm{~Bq} / \mathrm{S}$ & 0.69 & $\mathrm{pCi} / \mathrm{S}$ & Blank \\
\hline & $11 / 6 / 2007$ & $<0.032$ & $\mathrm{~Bq} / \mathrm{S}$ & $<0.87$ & $\mathrm{pCi} / \mathrm{S}$ & Blank \\
\hline & $12 / 4 / 2007$ & $<0.018$ & $\mathrm{~Bq} / \mathrm{S}$ & $<0.49$ & $\mathrm{pCi} / \mathrm{S}$ & Blank \\
\hline & $12 / 4 / 2007$ & $<0.037$ & $\mathrm{~Bq} / \mathrm{S}$ & $<1$ & $\mathrm{pCi} / \mathrm{S}$ & Blank \\
\hline & $1 / 8 / 2008$ & $<0.035$ & $\mathrm{~Bq} / \mathrm{S}$ & $<0.94$ & $\mathrm{pCi} / \mathrm{S}$ & Blank \\
\hline & $1 / 8 / 2008$ & $<0.02$ & $\mathrm{~Bq} / \mathrm{S}$ & $<0.53$ & $\mathrm{pCi} / \mathrm{S}$ & Blank \\
\hline
\end{tabular}




\begin{tabular}{|c|c|c|c|c|c|c|}
\hline I-125 & & $\mathrm{s}$ & & Conve & onal & \\
\hline Location & $\begin{array}{c}\text { Collection } \\
\text { Date }\end{array}$ & Result & Units & Result & Units & $\begin{array}{l}\text { QA } \\
\text { Type }\end{array}$ \\
\hline $55-128$ & $1 / 2 / 2007$ & 0.15 & $\mathrm{~Bq} / \mathrm{m}^{3}$ & 4.1 & $\mathrm{pCi} / \mathrm{m}^{3}$ & Sample \\
\hline & 2/6/2007 & 0.1 & $\mathrm{~Bq} / \mathrm{m}^{3}$ & 2.8 & $\mathrm{pCi} / \mathrm{m}^{3}$ & Sample \\
\hline & 3/7/2007 & 0.2 & $\mathrm{~Bq} / \mathrm{m}^{3}$ & 5.4 & $\mathrm{pCi} / \mathrm{m}^{3}$ & Sample \\
\hline & $4 / 3 / 2007$ & $<0.000022$ & $\mathrm{~Bq} / \mathrm{m}^{3}$ & $<0.00059$ & $\mathrm{pCi} / \mathrm{m}^{3}$ & Sample \\
\hline & $5 / 1 / 2007$ & 0.00017 & $\mathrm{~Bq} / \mathrm{m}^{3}$ & 0.0045 & $\mathrm{pCi} / \mathrm{m}^{3}$ & Sample \\
\hline & $6 / 5 / 2007$ & 0.00054 & $\mathrm{~Bq} / \mathrm{m}^{3}$ & 0.015 & $\mathrm{pCi} / \mathrm{m}^{3}$ & Sample \\
\hline & $7 / 3 / 2007$ & $<0.000014$ & $\mathrm{~Bq} / \mathrm{m}^{3}$ & $<0.00037$ & $\mathrm{pCi} / \mathrm{m}^{3}$ & Sample \\
\hline & $8 / 7 / 2007$ & $<0.000019$ & $\mathrm{~Bq} / \mathrm{m}^{3}$ & $<0.00051$ & $\mathrm{pCi} / \mathrm{m}^{3}$ & Sample \\
\hline & 9/4/2007 & $<0.000032$ & $\mathrm{Bg} / \mathrm{m}^{3}$ & $<0.00085$ & $\mathrm{pCi} / \mathrm{m}^{3}$ & Sample \\
\hline & $10 / 2 / 2007$ & $<0.000023$ & $\mathrm{~Bq} / \mathrm{m}^{3}$ & $<0.00061$ & $\mathrm{pCi} / \mathrm{m}^{3}$ & Sample \\
\hline & $11 / 6 / 2007$ & $<0.000033$ & $\mathrm{~Bq} / \mathrm{m}^{3}$ & $<0.0009$ & $\mathrm{pCi} / \mathrm{m}^{3}$ & Sample \\
\hline & $12 / 4 / 2007$ & $<0.000029$ & $\mathrm{~Bq} / \mathrm{m}^{3}$ & $<0.00079$ & $\mathrm{pCi} / \mathrm{m}^{3}$ & Sample \\
\hline & $1 / 8 / 2008$ & $<0.000019$ & $\mathrm{~Bq} / \mathrm{m}^{3}$ & $<0.0005$ & $\mathrm{pCi} / \mathrm{m}^{3}$ & Sample \\
\hline 55-128 Backup & 1/2/2007 & 0.00058 & $\mathrm{~Bq} / \mathrm{m}^{3}$ & 0.016 & $\mathrm{pCi} / \mathrm{m}^{3}$ & Sample \\
\hline & $2 / 6 / 2007$ & 0.00068 & $\mathrm{~Bq} / \mathrm{m}^{3}$ & 0.018 & $\mathrm{pCi} / \mathrm{m}^{3}$ & Sample \\
\hline & $3 / 7 / 2007$ & 0.00029 & $\mathrm{Bg} / \mathrm{m}^{3}$ & 0.0077 & $\mathrm{pCi} / \mathrm{m}^{3}$ & Sample \\
\hline & $4 / 3 / 2007$ & $<0.00004$ & $\mathrm{Bg} / \mathrm{m}^{3}$ & $<0.0011$ & $\mathrm{pCi} / \mathrm{m}^{3}$ & Sample \\
\hline & $5 / 1 / 2007$ & $<0.000023$ & $\mathrm{~Bq} / \mathrm{m}^{3}$ & $<0.00062$ & $\mathrm{pCi} / \mathrm{m}^{3}$ & Sample \\
\hline & $6 / 5 / 2007$ & $<0.000029$ & $\mathrm{~Bq} / \mathrm{m}^{3}$ & $<0.00078$ & $\mathrm{pCi} / \mathrm{m}^{3}$ & Sample \\
\hline & $7 / 3 / 2007$ & $<0.000019$ & $\mathrm{Bg} / \mathrm{m}^{3}$ & $<0.0005$ & $\mathrm{pCi} / \mathrm{m}^{3}$ & Sample \\
\hline & 8/7/2007 & $<0.000026$ & $\mathrm{~Bq} / \mathrm{m}^{3}$ & $<0.00069$ & $\mathrm{pCi} / \mathrm{m}^{3}$ & Sample \\
\hline & 9/4/2007 & $<0.000027$ & $\mathrm{~Bq} / \mathrm{m}^{3}$ & $<0.00074$ & $\mathrm{pCi} / \mathrm{m}^{3}$ & Sample \\
\hline & $10 / 2 / 2007$ & $<0.000026$ & $\mathrm{~Bq} / \mathrm{m}^{3}$ & $<0.00069$ & $\mathrm{pCi} / \mathrm{m}^{3}$ & Sample \\
\hline & $11 / 6 / 2007$ & $<0.000022$ & $\mathrm{~Bq} / \mathrm{m}^{3}$ & $<0.0006$ & $\mathrm{pCi} / \mathrm{m}^{3}$ & Sample \\
\hline & $12 / 4 / 2007$ & $<0.000017$ & $\mathrm{Bg} / \mathrm{m}^{3}$ & $<0.00045$ & $\mathrm{pCi} / \mathrm{m}^{3}$ & Sample \\
\hline & $1 / 8 / 2008$ & $<0.000021$ & $\mathrm{~Bq} / \mathrm{m}^{3}$ & $<0.00058$ & $\mathrm{pCi} / \mathrm{m}^{3}$ & Sample \\
\hline $55-128-\mathrm{COL}$ & $1 / 2 / 2007$ & 0.13 & $\mathrm{Bg} / \mathrm{m}^{3}$ & 3.4 & $\mathrm{pCi} / \mathrm{m}^{3}$ & Duplicate \\
\hline & 2/6/2007 & 0.072 & $\mathrm{Bg} / \mathrm{m}^{3}$ & 1.9 & $\mathrm{pCi} / \mathrm{m}^{3}$ & Duplicate \\
\hline & 3/7/2007 & 0.16 & $\mathrm{Bg} / \mathrm{m}^{3}$ & 4.3 & $\mathrm{pCi} / \mathrm{m}^{3}$ & Duplicate \\
\hline & 4/3/2007 & $<0.00022$ & $\mathrm{~Bq} / \mathrm{m}^{3}$ & $<0.0059$ & $\mathrm{pCi} / \mathrm{m}^{3}$ & Duplicate \\
\hline & $5 / 1 / 2007$ & $<0.00019$ & $\mathrm{~Bq} / \mathrm{m}^{3}$ & $<0.0051$ & $\mathrm{pCi} / \mathrm{m}^{3}$ & Duplicate \\
\hline
\end{tabular}




\begin{tabular}{|c|c|c|c|c|c|c|}
\hline $\mathrm{I}-125$ & & s & & Conve & ional & \\
\hline Location & $\begin{array}{c}\text { Collection } \\
\text { Date }\end{array}$ & Result & Units & Result & Units & $\begin{array}{c}\text { QA } \\
\text { Type }\end{array}$ \\
\hline $55-128-\mathrm{COL}$ & $6 / 5 / 2007$ & 0.00051 & $\mathrm{~Bq} / \mathrm{m}^{3}$ & 0.014 & $\mathrm{pCi} / \mathrm{m}^{3}$ & Duplicate \\
\hline & $7 / 3 / 2007$ & $<0.00027$ & $\mathrm{~Bq} / \mathrm{m}^{3}$ & $<0.0073$ & $\mathrm{pCi} / \mathrm{m}^{3}$ & Duplicate \\
\hline & 8/7/2007 & $<0.00018$ & $\mathrm{~Bq} / \mathrm{m}^{3}$ & $<0.0048$ & $\mathrm{pCi} / \mathrm{m}^{3}$ & Duplicate \\
\hline & 9/4/2007 & $<0.00022$ & $\mathrm{~Bq} / \mathrm{m}^{3}$ & $<0.0058$ & $\mathrm{pCi} / \mathrm{m}^{3}$ & Duplicate \\
\hline & 10/2/2007 & $<0.00024$ & $\mathrm{~Bq} / \mathrm{m}^{3}$ & $<0.0065$ & $\mathrm{pCi} / \mathrm{m}^{3}$ & Duplicate \\
\hline & $11 / 6 / 2007$ & $<0.00017$ & $\mathrm{~Bq} / \mathrm{m}^{3}$ & $<0.0046$ & $\mathrm{pCi} / \mathrm{m}^{3}$ & Duplicate \\
\hline & $12 / 4 / 2007$ & $<0.00021$ & $\mathrm{~Bq} / \mathrm{m}^{3}$ & $<0.0057$ & $\mathrm{pCi} / \mathrm{m}^{3}$ & Duplicate \\
\hline & $1 / 8 / 2008$ & $<0.00019$ & $\mathrm{~Bq} / \mathrm{m}^{3}$ & $<0.0051$ & $\mathrm{pCi} / \mathrm{m}^{3}$ & Duplicate \\
\hline 55-128-COL Backup & 1/2/2007 & 0.00063 & $\mathrm{~Bq} / \mathrm{m}^{3}$ & 0.017 & $\mathrm{pCi} / \mathrm{m}^{3}$ & Duplicate \\
\hline & 2/6/2007 & $<0.00024$ & $\mathrm{~Bq} / \mathrm{m}^{3}$ & $<0.0064$ & $\mathrm{pCi} / \mathrm{m}^{3}$ & Duplicate \\
\hline & $3 / 7 / 2007$ & 0.024 & $\mathrm{~Bq} / \mathrm{m}^{3}$ & 0.64 & $\mathrm{pCi} / \mathrm{m}^{3}$ & Duplicate \\
\hline & 4/3/2007 & $<0.00019$ & $\mathrm{~Bq} / \mathrm{m}^{3}$ & $<0.0052$ & $\mathrm{pCi} / \mathrm{m}^{3}$ & Duplicate \\
\hline & $5 / 1 / 2007$ & $<0.00022$ & $\mathrm{~Bq} / \mathrm{m}^{3}$ & $<0.006$ & $\mathrm{pCi} / \mathrm{m}^{3}$ & Duplicate \\
\hline & $6 / 5 / 2007$ & $<0.00021$ & $\mathrm{~Bq} / \mathrm{m}^{3}$ & $<0.0058$ & $\mathrm{pCi} / \mathrm{m}^{3}$ & Duplicate \\
\hline & $7 / 3 / 2007$ & $<0.0002$ & $\mathrm{~Bq} / \mathrm{m}^{3}$ & $<0.0053$ & $\mathrm{pCi} / \mathrm{m}^{3}$ & Duplicate \\
\hline & $8 / 7 / 2007$ & $<0.00016$ & $\mathrm{~Bq} / \mathrm{m}^{3}$ & $<0.0042$ & $\mathrm{pCi} / \mathrm{m}^{3}$ & Duplicate \\
\hline & 9/4/2007 & $<0.000026$ & $\mathrm{~Bq} / \mathrm{m}^{3}$ & $<0.0007$ & $\mathrm{pCi} / \mathrm{m}^{3}$ & Duplicate \\
\hline & $10 / 2 / 2007$ & $<0.000027$ & $\mathrm{~Bq} / \mathrm{m}^{3}$ & $<0.00072$ & $\mathrm{pCi} / \mathrm{m}^{3}$ & Duplicate \\
\hline & $11 / 6 / 2007$ & $<0.00002$ & $\mathrm{Bg} / \mathrm{m}^{3}$ & $<0.00054$ & $\mathrm{pCi} / \mathrm{m}^{3}$ & Duplicate \\
\hline & $12 / 4 / 2007$ & $<0.000026$ & $\mathrm{~Bq} / \mathrm{m}^{3}$ & $<0.00071$ & $\mathrm{pCi} / \mathrm{m}^{3}$ & Duplicate \\
\hline & 1/8/2008 & $<0.0002$ & $\mathrm{~Bq} / \mathrm{m}^{3}$ & $<0.0055$ & $\mathrm{pCi} / \mathrm{m}^{3}$ & Duplicate \\
\hline 85 Glovebox & 2/6/2007 & 0.000073 & $\mathrm{~Bq} / \mathrm{m}^{3}$ & 0.002 & $\mathrm{pCi} / \mathrm{m}^{3}$ & Sample \\
\hline & $5 / 1 / 2007$ & $<0.000027$ & $\mathrm{Bg} / \mathrm{m}^{3}$ & $<0.00073$ & $\mathrm{pCi} / \mathrm{m}^{3}$ & Sample \\
\hline & 9/4/2007 & $<0.000023$ & $\mathrm{~Bq} / \mathrm{m}^{3}$ & $<0.00063$ & $\mathrm{pCi} / \mathrm{m}^{3}$ & Sample \\
\hline & $11 / 6 / 2007$ & $<0.000024$ & $\mathrm{~Bq} / \mathrm{m}^{3}$ & $<0.00066$ & $\mathrm{pCi} / \mathrm{m}^{3}$ & Sample \\
\hline $85 \mathrm{Hood}$ & 2/6/2007 & 0.00013 & $\mathrm{~Bq} / \mathrm{m}^{3}$ & 0.0034 & $\mathrm{pCi} / \mathrm{m}^{3}$ & Sample \\
\hline & $5 / 1 / 2007$ & 0.000023 & $\mathrm{Bg} / \mathrm{m}^{3}$ & 0.00062 & $\mathrm{pCi} / \mathrm{m}^{3}$ & Sample \\
\hline & $8 / 7 / 2007$ & $<0.000013$ & $\mathrm{~Bq} / \mathrm{m}^{3}$ & $<0.00035$ & $\mathrm{pCi} / \mathrm{m}^{3}$ & Sample \\
\hline & $11 / 6 / 2007$ & $<0.000013$ & $\mathrm{~Bq} / \mathrm{m}^{3}$ & $<0.00034$ & $\mathrm{pCi} / \mathrm{m}^{3}$ & Sample \\
\hline TRAVEL BLANK & $1 / 2 / 2007$ & $<0.09$ & $\mathrm{~Bq} / \mathrm{S}$ & $<2.4$ & $\mathrm{pCi} / \mathrm{S}$ & Blank \\
\hline & $1 / 2 / 2007$ & $<0.021$ & $\mathrm{~Bq} / \mathrm{S}$ & $<0.56$ & $\mathrm{pCi} / \mathrm{S}$ & Blank \\
\hline & $2 / 6 / 2007$ & $<0.013$ & $\mathrm{~Bq} / \mathrm{S}$ & $<0.35$ & $\mathrm{pCi} / \mathrm{S}$ & Blank \\
\hline
\end{tabular}




\begin{tabular}{|c|c|c|c|c|c|c|}
\hline I-125 & & & & Conv & onal & \\
\hline Location & $\begin{array}{c}\text { Collection } \\
\text { Date }\end{array}$ & Result & Units & Result & Units & $\begin{array}{c}\text { QA } \\
\text { Type }\end{array}$ \\
\hline TRAVEL BLANK & 2/6/2007 & $<0.11$ & $\mathrm{~Bq} / \mathrm{S}$ & $<3.1$ & $\mathrm{pCi} / \mathrm{S}$ & Blank \\
\hline & $3 / 6 / 2007$ & $<0.1$ & $\mathrm{~Bq} / \mathrm{S}$ & $<2.8$ & $\mathrm{pCi} / \mathrm{S}$ & Blank \\
\hline & $3 / 6 / 2007$ & 0.011 & $\mathrm{~Bq} / \mathrm{S}$ & 0.3 & $\mathrm{pCi} / \mathrm{S}$ & Blank \\
\hline & $4 / 3 / 2007$ & $<0.14$ & $\mathrm{~Bq} / \mathrm{S}$ & $<3.8$ & $\mathrm{pCi} / \mathrm{S}$ & Blank \\
\hline & $4 / 3 / 2007$ & $<0.012$ & $\mathrm{~Bq} / \mathrm{S}$ & $<0.33$ & $\mathrm{pCi} / \mathrm{S}$ & Blank \\
\hline & $5 / 1 / 2007$ & $<0.083$ & $\mathrm{~Bq} / \mathrm{S}$ & $<2.2$ & $\mathrm{pCi} / \mathrm{S}$ & Blank \\
\hline & $5 / 1 / 2007$ & $<0.0099$ & $\mathrm{~Bq} / \mathrm{S}$ & $<0.27$ & $\mathrm{pCi} / \mathrm{S}$ & Blank \\
\hline & $6 / 5 / 2007$ & $<0.022$ & $\mathrm{~Bq} / \mathrm{S}$ & $<0.58$ & $\mathrm{pCi} / \mathrm{S}$ & Blank \\
\hline & $6 / 5 / 2007$ & $<0.088$ & $\mathrm{~Bq} / \mathrm{S}$ & $<2.4$ & $\mathrm{pCi} / \mathrm{S}$ & Blank \\
\hline & $7 / 3 / 2007$ & $<0.091$ & $\mathrm{~Bq} / \mathrm{S}$ & $<2.5$ & $\mathrm{pCi} / \mathrm{S}$ & Blank \\
\hline & $7 / 3 / 2007$ & $<0.014$ & $\mathrm{~Bq} / \mathrm{S}$ & $<0.39$ & $\mathrm{pCi} / \mathrm{S}$ & Blank \\
\hline & $8 / 7 / 2007$ & $<0.014$ & $\mathrm{~Bq} / \mathrm{S}$ & $<0.38$ & $\mathrm{pCi} / \mathrm{S}$ & Blank \\
\hline & $8 / 7 / 2007$ & $<0.091$ & $\mathrm{~Bq} / \mathrm{S}$ & $<2.5$ & $\mathrm{pCi} / \mathrm{S}$ & Blank \\
\hline & $9 / 4 / 2007$ & $<0.014$ & $\mathrm{~Bq} / \mathrm{S}$ & $<0.37$ & $\mathrm{pCi} / \mathrm{S}$ & Blank \\
\hline & $9 / 4 / 2007$ & $<0.1$ & $\mathrm{~Bq} / \mathrm{S}$ & $<2.7$ & $\mathrm{pCi} / \mathrm{S}$ & Blank \\
\hline & $10 / 2 / 2007$ & $<0.0097$ & $\mathrm{~Bq} / \mathrm{S}$ & $<0.26$ & $\mathrm{pCi} / \mathrm{S}$ & Blank \\
\hline & $10 / 2 / 2007$ & $<0.096$ & $\mathrm{~Bq} / \mathrm{S}$ & $<2.6$ & $\mathrm{pCi} / \mathrm{S}$ & Blank \\
\hline & $11 / 6 / 2007$ & $<0.012$ & $\mathrm{~Bq} / \mathrm{S}$ & $<0.32$ & $\mathrm{pCi} / \mathrm{S}$ & Blank \\
\hline & $11 / 6 / 2007$ & $<0.08$ & $\mathrm{~Bq} / \mathrm{S}$ & $<2.2$ & $\mathrm{pCi} / \mathrm{S}$ & Blank \\
\hline & $12 / 4 / 2007$ & $<0.011$ & $\mathrm{~Bq} / \mathrm{S}$ & $<0.3$ & $\mathrm{pCi} / \mathrm{S}$ & Blank \\
\hline & $12 / 4 / 2007$ & $<0.0078$ & $\mathrm{~Bq} / \mathrm{S}$ & $<0.21$ & $\mathrm{pCi} / \mathrm{S}$ & Blank \\
\hline & 1/8/2008 & $<0.0084$ & $\mathrm{~Bq} / \mathrm{S}$ & $<0.23$ & $\mathrm{pCi} / \mathrm{S}$ & Blank \\
\hline & $1 / 8 / 2008$ & $<0.091$ & $\mathrm{~Bq} / \mathrm{S}$ & $<2.5$ & $\mathrm{pCi} / \mathrm{S}$ & Blank \\
\hline
\end{tabular}




\begin{tabular}{|c|c|c|c|c|c|c|}
\hline \multirow{2}{*}{$\begin{array}{r}\text { Tritium } \\
\text { Location }\end{array}$} & \multirow[b]{2}{*}{$\begin{array}{c}\text { Collection } \\
\text { Date }\end{array}$} & \multicolumn{2}{|c|}{ S.I. } & \multicolumn{2}{|c|}{ Conventional } & \multirow[b]{2}{*}{$\begin{array}{c}\text { QA } \\
\text { Type }\end{array}$} \\
\hline & & Result & Units & Result & Units & \\
\hline \multirow[t]{7}{*}{85 Glovebox } & 2/6/2007 & 0.43 & $\mathrm{~Bq} / \mathrm{m}^{3}$ & 12 & $\mathrm{pCi} / \mathrm{m}^{3}$ & Sample \\
\hline & $2 / 6 / 2007$ & 0.69 & $\mathrm{~Bq} / \mathrm{m}^{3}$ & 19 & $\mathrm{pCi} / \mathrm{m}^{3}$ & Split \\
\hline & $5 / 1 / 2007$ & $<0.28$ & $\mathrm{~Bq} / \mathrm{m}^{3}$ & $<7.6$ & $\mathrm{pCi} / \mathrm{m}^{3}$ & Sample \\
\hline & $8 / 7 / 2007$ & 0.16 & $\mathrm{Bg} / \mathrm{m}^{3}$ & 4.4 & $\mathrm{pCi} / \mathrm{m}^{3}$ & Sample \\
\hline & $9 / 4 / 2007$ & 0.42 & $\mathrm{~Bq} / \mathrm{m}^{3}$ & 11 & $\mathrm{pCi} / \mathrm{m}^{3}$ & Sample \\
\hline & $10 / 2 / 2007$ & $<0.22$ & $\mathrm{~Bq} / \mathrm{m}^{3}$ & $<5.9$ & $\mathrm{pCi} / \mathrm{m}^{3}$ & Sample \\
\hline & $11 / 6 / 2007$ & 0.94 & $\mathrm{~Bq} / \mathrm{m}^{3}$ & 25 & $\mathrm{pCi} / \mathrm{m}^{3}$ & Sample \\
\hline \multirow[t]{7}{*}{$85 \mathrm{Hood}$} & $2 / 6 / 2007$ & 1.5 & $\mathrm{Bg} / \mathrm{m}^{3}$ & 41 & $\mathrm{pCi} / \mathrm{m}^{3}$ & Sample \\
\hline & $2 / 6 / 2007$ & 2.9 & $\mathrm{~Bq} / \mathrm{m}^{3}$ & 78 & $\mathrm{pCi} / \mathrm{m}^{3}$ & Split \\
\hline & $5 / 8 / 2007$ & 1.3 & $\mathrm{~Bq} / \mathrm{m}^{3}$ & 35 & $\mathrm{pCi} / \mathrm{m}^{3}$ & Sample \\
\hline & $8 / 7 / 2007$ & 1 & $\mathrm{~Bq} / \mathrm{m}^{3}$ & 28 & $\mathrm{pCi} / \mathrm{m}^{3}$ & Sample \\
\hline & $9 / 4 / 2007$ & 2.1 & $\mathrm{Bg} / \mathrm{m}^{3}$ & 58 & $\mathrm{pCi} / \mathrm{m}^{3}$ & Sample \\
\hline & $10 / 2 / 2007$ & 2.9 & $\mathrm{~Bq} / \mathrm{m}^{3}$ & 78 & $\mathrm{pCi} / \mathrm{m}^{3}$ & Sample \\
\hline & $11 / 6 / 2007$ & 170 & $\mathrm{~Bq} / \mathrm{m}^{3}$ & 4600 & $\mathrm{pCi} / \mathrm{m}^{3}$ & Sample \\
\hline \multirow[t]{3}{*}{$\begin{array}{l}\text { NTLF Hillside Stack } \\
\text { Drain }\end{array}$} & $1 / 16 / 2007$ & 39000 & $\mathrm{~Bq} / \mathrm{L}$ & 1000000 & $\mathrm{pCi} / \mathrm{L}$ & Sample \\
\hline & $1 / 16 / 2007$ & 70000 & $\mathrm{~Bq} / \mathrm{L}$ & 1900000 & $\mathrm{pCi} / \mathrm{L}$ & Split \\
\hline & $1 / 16 / 2007$ & 300000 & $\mathrm{~Bq} / \mathrm{L}$ & 8000000 & $\mathrm{pCi} / \mathrm{L}$ & Split \\
\hline \multirow[t]{7}{*}{ TRAVEL BLANK } & 2/6/2007 & $<0.25$ & $\mathrm{~Bq} / \mathrm{S}$ & $<6.8$ & $\mathrm{pCi} / \mathrm{S}$ & Blank \\
\hline & 2/6/2007 & $<0.53$ & $\mathrm{~Bq} / \mathrm{S}$ & $<14$ & $\mathrm{pCi} / \mathrm{S}$ & Blank \\
\hline & $5 / 1 / 2007$ & $<0.2$ & $\mathrm{~Bq} / \mathrm{S}$ & $<5.4$ & $\mathrm{pCi} / \mathrm{S}$ & Blank \\
\hline & $8 / 7 / 2007$ & $<0.22$ & $\mathrm{~Bq} / \mathrm{S}$ & $<6$ & $\mathrm{pCi} / \mathrm{S}$ & Blank \\
\hline & 9/4/2007 & $<0.22$ & $\mathrm{~Bq} / \mathrm{S}$ & $<5.9$ & $\mathrm{pCi} / \mathrm{S}$ & Blank \\
\hline & $10 / 2 / 2007$ & $<0.27$ & $\mathrm{~Bq} / \mathrm{S}$ & $<7.4$ & $\mathrm{pCi} / \mathrm{S}$ & Blank \\
\hline & $11 / 6 / 2007$ & $<0.19$ & $\mathrm{~Bq} / \mathrm{S}$ & $<5.1$ & $\mathrm{pCi} / \mathrm{S}$ & Blank \\
\hline
\end{tabular}




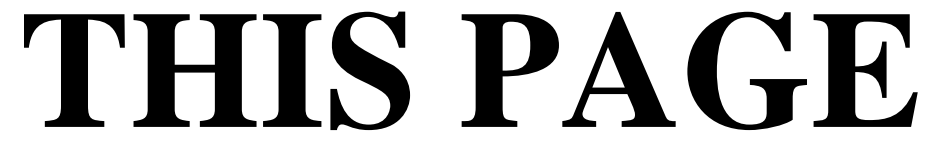

INTENTIONALLY LEFT BLANK 


\begin{tabular}{|c|c|c|c|c|c|c|}
\hline \multicolumn{2}{|c|}{ Gross alpha } & \multicolumn{2}{|c|}{ S.I. } & \multicolumn{2}{|c|}{ Conventional } & \multirow[b]{2}{*}{$\begin{array}{l}\text { QA } \\
\text { Type }\end{array}$} \\
\hline Location & $\begin{array}{c}\text { Collection } \\
\text { Date }\end{array}$ & Result & Units & Result & Units & \\
\hline \multirow[t]{13}{*}{ ENV-44 } & $1 / 9 / 2007$ & 0.00011 & $\mathrm{~Bq} / \mathrm{m}^{3}$ & 0.0029 & $\mathrm{pCi} / \mathrm{m}^{3}$ & Sample \\
\hline & $2 / 5 / 2007$ & 0.000073 & $\mathrm{~Bq} / \mathrm{m}^{3}$ & 0.002 & $\mathrm{pCi} / \mathrm{m}^{3}$ & Sample \\
\hline & $3 / 5 / 2007$ & 0.000047 & $\mathrm{~Bq} / \mathrm{m}^{3}$ & 0.0013 & $\mathrm{pCi} / \mathrm{m}^{3}$ & Sample \\
\hline & $4 / 2 / 2007$ & 0.000054 & $\mathrm{~Bq} / \mathrm{m}^{3}$ & 0.0015 & $\mathrm{pCi} / \mathrm{m}^{3}$ & Sample \\
\hline & $5 / 7 / 2007$ & 0.000013 & $\mathrm{~Bq} / \mathrm{m}^{3}$ & 0.00036 & $\mathrm{pCi} / \mathrm{m}^{3}$ & Sample \\
\hline & $6 / 4 / 2007$ & 0.000048 & $\mathrm{~Bq} / \mathrm{m}^{3}$ & 0.0013 & $\mathrm{pCi} / \mathrm{m}^{3}$ & Sample \\
\hline & $7 / 2 / 2007$ & 0.000028 & $\mathrm{~Bq} / \mathrm{m}^{3}$ & 0.00074 & $\mathrm{pCi} / \mathrm{m}^{3}$ & Sample \\
\hline & $8 / 6 / 2007$ & 0.0000071 & $\mathrm{~Bq} / \mathrm{m}^{3}$ & 0.00019 & $\mathrm{pCi} / \mathrm{m}^{3}$ & Sample \\
\hline & $9 / 4 / 2007$ & $<0.0000042$ & $\mathrm{~Bq} / \mathrm{m}^{3}$ & $<0.00011$ & $\mathrm{pCi} / \mathrm{m}^{3}$ & Sample \\
\hline & $10 / 3 / 2007$ & 0.000044 & $\mathrm{~Bq} / \mathrm{m}^{3}$ & 0.0012 & $\mathrm{pCi} / \mathrm{m}^{3}$ & Sample \\
\hline & $11 / 5 / 2007$ & 0.000039 & $\mathrm{~Bq} / \mathrm{m}^{3}$ & 0.001 & $\mathrm{pCi} / \mathrm{m}^{3}$ & Sample \\
\hline & $12 / 3 / 2007$ & 0.000085 & $\mathrm{~Bq} / \mathrm{m}^{3}$ & 0.0023 & $\mathrm{pCi} / \mathrm{m}^{3}$ & Sample \\
\hline & $1 / 7 / 2008$ & 0.000029 & $\mathrm{~Bq} / \mathrm{m}^{3}$ & 0.00077 & $\mathrm{pCi} / \mathrm{m}^{3}$ & Sample \\
\hline \multirow[t]{13}{*}{ ENV-44-COL } & $1 / 9 / 2007$ & 0.000025 & $\mathrm{~Bq} / \mathrm{m}^{3}$ & 0.00066 & $\mathrm{pCi} / \mathrm{m}^{3}$ & Duplicate \\
\hline & $2 / 5 / 2007$ & 0.00004 & $\mathrm{~Bq} / \mathrm{m}^{3}$ & 0.0011 & $\mathrm{pCi} / \mathrm{m}^{3}$ & Duplicate \\
\hline & $3 / 5 / 2007$ & $<0.000013$ & $\mathrm{~Bq} / \mathrm{m}^{3}$ & $<0.00034$ & $\mathrm{pCi} / \mathrm{m}^{3}$ & Duplicate \\
\hline & $4 / 2 / 2007$ & 0.000016 & $\mathrm{~Bq} / \mathrm{m}^{3}$ & 0.00042 & $\mathrm{pCi} / \mathrm{m}^{3}$ & Duplicate \\
\hline & $5 / 7 / 2007$ & 0.000012 & $\mathrm{~Bq} / \mathrm{m}^{3}$ & 0.00032 & $\mathrm{pCi} / \mathrm{m}^{3}$ & Duplicate \\
\hline & $6 / 4 / 2007$ & 0.000011 & $\mathrm{~Bq} / \mathrm{m}^{3}$ & 0.00029 & $\mathrm{pCi} / \mathrm{m}^{3}$ & Duplicate \\
\hline & $7 / 2 / 2007$ & $<0.000011$ & $\mathrm{~Bq} / \mathrm{m}^{3}$ & $<0.00029$ & $\mathrm{pCi} / \mathrm{m}^{3}$ & Duplicate \\
\hline & $8 / 6 / 2007$ & $<0.0000094$ & $\mathrm{~Bq} / \mathrm{m}^{3}$ & $<0.00025$ & $\mathrm{pCi} / \mathrm{m}^{3}$ & Duplicate \\
\hline & $9 / 4 / 2007$ & 0.000031 & $\mathrm{~Bq} / \mathrm{m}^{3}$ & 0.00085 & $\mathrm{pCi} / \mathrm{m}^{3}$ & Duplicate \\
\hline & $10 / 3 / 2007$ & $<0.000012$ & $\mathrm{~Bq} / \mathrm{m}^{3}$ & $<0.00034$ & $\mathrm{pCi} / \mathrm{m}^{3}$ & Duplicate \\
\hline & $11 / 5 / 2007$ & 0.000023 & $\mathrm{~Bq} / \mathrm{m}^{3}$ & 0.00062 & $\mathrm{pCi} / \mathrm{m}^{3}$ & Duplicate \\
\hline & $12 / 3 / 2007$ & 0.000062 & $\mathrm{~Bq} / \mathrm{m}^{3}$ & 0.0017 & $\mathrm{pCi} / \mathrm{m}^{3}$ & Duplicate \\
\hline & 1/7/2008 & 0.000016 & $\mathrm{~Bq} / \mathrm{m}^{3}$ & 0.00044 & $\mathrm{pCi} / \mathrm{m}^{3}$ & Duplicate \\
\hline \multirow[t]{5}{*}{ ENV-83 } & $1 / 9 / 2007$ & 0.00011 & $\mathrm{~Bq} / \mathrm{m}^{3}$ & 0.003 & $\mathrm{pCi} / \mathrm{m}^{3}$ & Sample \\
\hline & $2 / 5 / 2007$ & 0.000079 & $\mathrm{~Bq} / \mathrm{m}^{3}$ & 0.0021 & $\mathrm{pCi} / \mathrm{m}^{3}$ & Sample \\
\hline & $3 / 5 / 2007$ & 0.000053 & $\mathrm{~Bq} / \mathrm{m}^{3}$ & 0.0014 & $\mathrm{pCi} / \mathrm{m}^{3}$ & Sample \\
\hline & $4 / 2 / 2007$ & 0.000069 & $\mathrm{~Bq} / \mathrm{m}^{3}$ & 0.0019 & $\mathrm{pCi} / \mathrm{m}^{3}$ & Sample \\
\hline & $5 / 7 / 2007$ & 0.0000085 & $\mathrm{~Bq} / \mathrm{m}^{3}$ & 0.00023 & $\mathrm{pCi} / \mathrm{m}^{3}$ & Sample \\
\hline
\end{tabular}




\begin{tabular}{|c|c|c|c|c|c|c|}
\hline \multicolumn{2}{|c|}{ Gross alpha } & \multicolumn{2}{|c|}{ S.I. } & \multicolumn{2}{|c|}{ Conventional } & \multirow[b]{2}{*}{$\begin{array}{c}\text { QA } \\
\text { Type }\end{array}$} \\
\hline Location & $\begin{array}{c}\text { Collection } \\
\text { Date }\end{array}$ & Result & Units & Result & Units & \\
\hline \multirow[t]{8}{*}{ ENV-83 } & 6/4/2007 & 0.000049 & $\mathrm{~Bq} / \mathrm{m}^{3}$ & 0.0013 & $\mathrm{pCi} / \mathrm{m}^{3}$ & Sample \\
\hline & $7 / 2 / 2007$ & 0.000036 & $\mathrm{~Bq} / \mathrm{m}^{3}$ & 0.00098 & $\mathrm{pCi} / \mathrm{m}^{3}$ & Sample \\
\hline & $8 / 6 / 2007$ & 0.000015 & $\mathrm{~Bq} / \mathrm{m}^{3}$ & 0.0004 & $\mathrm{pCi} / \mathrm{m}^{3}$ & Sample \\
\hline & 9/4/2007 & 0.000051 & $\mathrm{~Bq} / \mathrm{m}^{3}$ & 0.0014 & $\mathrm{pCi} / \mathrm{m}^{3}$ & Sample \\
\hline & $10 / 3 / 2007$ & 0.00006 & $\mathrm{~Bq} / \mathrm{m}^{3}$ & 0.0016 & $\mathrm{pCi} / \mathrm{m}^{3}$ & Sample \\
\hline & $11 / 5 / 2007$ & 0.00005 & $\mathrm{~Bq} / \mathrm{m}^{3}$ & 0.0013 & $\mathrm{pCi} / \mathrm{m}^{3}$ & Sample \\
\hline & $12 / 3 / 2007$ & 0.00011 & $\mathrm{~Bq} / \mathrm{m}^{3}$ & 0.0029 & $\mathrm{pCi} / \mathrm{m}^{3}$ & Sample \\
\hline & $1 / 7 / 2008$ & 0.000027 & $\mathrm{~Bq} / \mathrm{m}^{3}$ & 0.00074 & $\mathrm{pCi} / \mathrm{m}^{3}$ & Sample \\
\hline \multirow[t]{13}{*}{ ENV-B13A } & $1 / 9 / 2007$ & 0.00012 & $\mathrm{Bg} / \mathrm{m}^{3}$ & 0.0031 & $\mathrm{pCi} / \mathrm{m}^{3}$ & Sample \\
\hline & $2 / 5 / 2007$ & 0.000069 & $\mathrm{~Bq} / \mathrm{m}^{3}$ & 0.0019 & $\mathrm{pCi} / \mathrm{m}^{3}$ & Sample \\
\hline & $3 / 5 / 2007$ & 0.000056 & $\mathrm{~Bq} / \mathrm{m}^{3}$ & 0.0015 & $\mathrm{pCi} / \mathrm{m}^{3}$ & Sample \\
\hline & $4 / 2 / 2007$ & 0.000068 & $\mathrm{~Bq} / \mathrm{m}^{3}$ & 0.0018 & $\mathrm{pCi} / \mathrm{m}^{3}$ & Sample \\
\hline & $5 / 7 / 2007$ & 0.000018 & $\mathrm{~Bq} / \mathrm{m}^{3}$ & 0.00047 & $\mathrm{pCi} / \mathrm{m}^{3}$ & Sample \\
\hline & $6 / 4 / 2007$ & 0.000048 & $\mathrm{~Bq} / \mathrm{m}^{3}$ & 0.0013 & $\mathrm{pCi} / \mathrm{m}^{3}$ & Sample \\
\hline & $7 / 2 / 2007$ & 0.000018 & $\mathrm{~Bq} / \mathrm{m}^{3}$ & 0.0005 & $\mathrm{pCi} / \mathrm{m}^{3}$ & Sample \\
\hline & $8 / 6 / 2007$ & 0.000014 & $\mathrm{~Bq} / \mathrm{m}^{3}$ & 0.00037 & $\mathrm{pCi} / \mathrm{m}^{3}$ & Sample \\
\hline & 9/4/2007 & 0.000054 & $\mathrm{Bg} / \mathrm{m}^{3}$ & 0.0014 & $\mathrm{pCi} / \mathrm{m}^{3}$ & Sample \\
\hline & $10 / 3 / 2007$ & 0.000055 & $\mathrm{Bg} / \mathrm{m}^{3}$ & 0.0015 & $\mathrm{pCi} / \mathrm{m}^{3}$ & Sample \\
\hline & $11 / 5 / 2007$ & 0.000042 & $\mathrm{~Bq} / \mathrm{m}^{3}$ & 0.0011 & $\mathrm{pCi} / \mathrm{m}^{3}$ & Sample \\
\hline & $12 / 3 / 2007$ & 0.00012 & $\mathrm{~Bq} / \mathrm{m}^{3}$ & 0.0033 & $\mathrm{pCi} / \mathrm{m}^{3}$ & Sample \\
\hline & $1 / 7 / 2008$ & 0.00002 & $\mathrm{~Bq} / \mathrm{m}^{3}$ & 0.00053 & $\mathrm{pCi} / \mathrm{m}^{3}$ & Sample \\
\hline \multirow[t]{11}{*}{ ENV-B13C } & $1 / 9 / 2007$ & 0.000083 & $\mathrm{Bg} / \mathrm{m}^{3}$ & 0.0023 & $\mathrm{pCi} / \mathrm{m}^{3}$ & Sample \\
\hline & $2 / 5 / 2007$ & 0.00012 & $\mathrm{~Bq} / \mathrm{m}^{3}$ & 0.0031 & $\mathrm{pCi} / \mathrm{m}^{3}$ & Sample \\
\hline & $3 / 5 / 2007$ & 0.000058 & $\mathrm{~Bq} / \mathrm{m}^{3}$ & 0.0016 & $\mathrm{pCi} / \mathrm{m}^{3}$ & Sample \\
\hline & 4/2/2007 & 0.000067 & $\mathrm{~Bq} / \mathrm{m}^{3}$ & 0.0018 & $\mathrm{pCi} / \mathrm{m}^{3}$ & Sample \\
\hline & $5 / 7 / 2007$ & 0.000019 & $\mathrm{Bg} / \mathrm{m}^{3}$ & 0.00051 & $\mathrm{pCi} / \mathrm{m}^{3}$ & Sample \\
\hline & $6 / 4 / 2007$ & 0.000049 & $\mathrm{Bg} / \mathrm{m}^{3}$ & 0.0013 & $\mathrm{pCi} / \mathrm{m}^{3}$ & Sample \\
\hline & $7 / 2 / 2007$ & 0.000016 & $\mathrm{~Bq} / \mathrm{m}^{3}$ & 0.00043 & $\mathrm{pCi} / \mathrm{m}^{3}$ & Sample \\
\hline & $8 / 6 / 2007$ & 0.000011 & $\mathrm{~Bq} / \mathrm{m}^{3}$ & 0.00029 & $\mathrm{pCi} / \mathrm{m}^{3}$ & Sample \\
\hline & 9/4/2007 & 0.000043 & $\mathrm{Bg} / \mathrm{m}^{3}$ & 0.0012 & $\mathrm{pCi} / \mathrm{m}^{3}$ & Sample \\
\hline & $10 / 3 / 2007$ & 0.000047 & $\mathrm{~Bq} / \mathrm{m}^{3}$ & 0.0013 & $\mathrm{pCi} / \mathrm{m}^{3}$ & Sample \\
\hline & $11 / 5 / 2007$ & 0.000047 & $\mathrm{~Bq} / \mathrm{m}^{3}$ & 0.0013 & $\mathrm{pCi} / \mathrm{m}^{3}$ & Sample \\
\hline
\end{tabular}




\begin{tabular}{|c|c|c|c|c|c|c|}
\hline \multicolumn{2}{|c|}{ Gross alpha } & \multicolumn{2}{|c|}{ S.I. } & \multicolumn{2}{|c|}{ Conventional } & \multirow[b]{2}{*}{$\begin{array}{c}\text { QA } \\
\text { Type }\end{array}$} \\
\hline Location & $\begin{array}{c}\text { Collection } \\
\text { Date }\end{array}$ & Result & Units & Result & Units & \\
\hline \multirow[t]{2}{*}{ ENV-B13C } & $12 / 3 / 2007$ & 0.000094 & $\mathrm{Bg} / \mathrm{m}^{3}$ & 0.0025 & $\mathrm{pCi} / \mathrm{m}^{3}$ & Sample \\
\hline & $1 / 7 / 2008$ & 0.000014 & $\mathrm{Bg} / \mathrm{m}^{3}$ & 0.00038 & $\mathrm{pCi} / \mathrm{m}^{3}$ & Sample \\
\hline \multirow[t]{26}{*}{ LOT BLANK } & $1 / 9 / 2007$ & $<0.02$ & $\mathrm{~Bq} / \mathrm{S}$ & $<0.54$ & $\mathrm{pCi} / \mathrm{S}$ & Blank \\
\hline & $1 / 9 / 2007$ & $<0.028$ & $\mathrm{~Bq} / \mathrm{S}$ & $<0.76$ & $\mathrm{pCi} / \mathrm{S}$ & Blank \\
\hline & 2/5/2007 & $<0.026$ & $\mathrm{~Bq} / \mathrm{S}$ & $<0.7$ & $\mathrm{pCi} / \mathrm{S}$ & Blank \\
\hline & 2/5/2007 & $<0.0083$ & $\mathrm{~Bq} / \mathrm{S}$ & $<0.22$ & $\mathrm{pCi} / \mathrm{S}$ & Blank \\
\hline & $3 / 5 / 2007$ & $<0.0077$ & $\mathrm{~Bq} / \mathrm{S}$ & $<0.21$ & $\mathrm{pCi} / \mathrm{S}$ & Blank \\
\hline & $3 / 5 / 2007$ & $<0.026$ & $\mathrm{~Bq} / \mathrm{S}$ & $<0.71$ & $\mathrm{pCi} / \mathrm{S}$ & Blank \\
\hline & $4 / 2 / 2007$ & 0.029 & $\mathrm{~Bq} / \mathrm{S}$ & 0.79 & $\mathrm{pCi} / \mathrm{S}$ & Blank \\
\hline & 4/2/2007 & $<0.0079$ & $\mathrm{~Bq} / \mathrm{S}$ & $<0.21$ & $\mathrm{pCi} / \mathrm{S}$ & Blank \\
\hline & $5 / 7 / 2007$ & $<0.024$ & $\mathrm{~Bq} / \mathrm{S}$ & $<0.64$ & $\mathrm{pCi} / \mathrm{S}$ & Blank \\
\hline & $5 / 7 / 2007$ & $<0.009$ & $\mathrm{~Bq} / \mathrm{S}$ & $<0.24$ & $\mathrm{pCi} / \mathrm{S}$ & Blank \\
\hline & 6/4/2007 & $<0.022$ & $\mathrm{~Bq} / \mathrm{S}$ & $<0.59$ & $\mathrm{pCi} / \mathrm{S}$ & Blank \\
\hline & $6 / 4 / 2007$ & $<0.012$ & $\mathrm{~Bq} / \mathrm{S}$ & $<0.32$ & $\mathrm{pCi} / \mathrm{S}$ & Blank \\
\hline & $7 / 2 / 2007$ & $<0.021$ & $\mathrm{~Bq} / \mathrm{S}$ & $<0.58$ & $\mathrm{pCi} / \mathrm{S}$ & Blank \\
\hline & $7 / 2 / 2007$ & $<0.014$ & $\mathrm{~Bq} / \mathrm{S}$ & $<0.38$ & $\mathrm{pCi} / \mathrm{S}$ & Blank \\
\hline & $8 / 6 / 2007$ & $<0.024$ & $\mathrm{~Bq} / \mathrm{S}$ & $<0.65$ & $\mathrm{pCi} / \mathrm{S}$ & Blank \\
\hline & $8 / 6 / 2007$ & $<0.013$ & $\mathrm{~Bq} / \mathrm{S}$ & $<0.35$ & $\mathrm{pCi} / \mathrm{S}$ & Blank \\
\hline & 9/4/2007 & $<0.024$ & $\mathrm{~Bq} / \mathrm{S}$ & $<0.64$ & $\mathrm{pCi} / \mathrm{S}$ & Blank \\
\hline & 9/4/2007 & 0.021 & $\mathrm{~Bq} / \mathrm{S}$ & 0.57 & $\mathrm{pCi} / \mathrm{S}$ & Blank \\
\hline & $10 / 3 / 2007$ & $<0.01$ & $\mathrm{~Bq} / \mathrm{S}$ & $<0.27$ & $\mathrm{pCi} / \mathrm{S}$ & Blank \\
\hline & $10 / 3 / 2007$ & $<0.025$ & $\mathrm{~Bq} / \mathrm{S}$ & $<0.68$ & $\mathrm{pCi} / \mathrm{S}$ & Blank \\
\hline & $11 / 5 / 2007$ & $<0.02$ & $\mathrm{~Bq} / \mathrm{S}$ & $<0.53$ & $\mathrm{pCi} / \mathrm{S}$ & Blank \\
\hline & $11 / 5 / 2007$ & $<0.014$ & $\mathrm{~Bq} / \mathrm{S}$ & $<0.37$ & $\mathrm{pCi} / \mathrm{S}$ & Blank \\
\hline & $12 / 3 / 2007$ & $<0.012$ & $\mathrm{~Bq} / \mathrm{S}$ & $<0.32$ & $\mathrm{pCi} / \mathrm{S}$ & Blank \\
\hline & $12 / 3 / 2007$ & $<0.023$ & $\mathrm{~Bq} / \mathrm{S}$ & $<0.63$ & $\mathrm{pCi} / \mathrm{S}$ & Blank \\
\hline & $1 / 7 / 2008$ & $<0.012$ & $\mathrm{~Bq} / \mathrm{S}$ & $<0.32$ & $\mathrm{pCi} / \mathrm{S}$ & Blank \\
\hline & $1 / 7 / 2008$ & $<0.02$ & $\mathrm{Bg} / \mathrm{S}$ & $<0.55$ & $\mathrm{pCi} / \mathrm{S}$ & Blank \\
\hline \multirow[t]{7}{*}{ TRAVEL BLANK } & $1 / 9 / 2007$ & $<0.027$ & $\mathrm{~Bq} / \mathrm{S}$ & $<0.74$ & $\mathrm{pCi} / \mathrm{S}$ & Blank \\
\hline & 1/9/2007 & $<0.012$ & $\mathrm{~Bq} / \mathrm{S}$ & $<0.32$ & $\mathrm{pCi} / \mathrm{S}$ & Blank \\
\hline & $2 / 5 / 2007$ & $<0.0099$ & $\mathrm{~Bq} / \mathrm{S}$ & $<0.27$ & $\mathrm{pCi} / \mathrm{S}$ & Blank \\
\hline & $2 / 5 / 2007$ & $<0.026$ & $\mathrm{~Bq} / \mathrm{S}$ & $<0.69$ & $\mathrm{pCi} / \mathrm{S}$ & Blank \\
\hline & $3 / 5 / 2007$ & $<0.026$ & $\mathrm{~Bq} / \mathrm{S}$ & $<0.7$ & $\mathrm{pCi} / \mathrm{S}$ & Blank \\
\hline & $3 / 5 / 2007$ & $<0.0074$ & $\mathrm{~Bq} / \mathrm{S}$ & $<0.2$ & $\mathrm{pCi} / \mathrm{S}$ & Blank \\
\hline & 4/2/2007 & $<0.011$ & $\mathrm{~Bq} / \mathrm{S}$ & $<0.29$ & $\mathrm{pCi} / \mathrm{S}$ & Blank \\
\hline
\end{tabular}




\begin{tabular}{|c|c|c|c|c|c|c|}
\hline \multicolumn{2}{|c|}{ Gross alpha } & \multicolumn{2}{|c|}{ S.I. } & \multicolumn{2}{|c|}{ Conventional } & \multirow[b]{2}{*}{$\begin{array}{l}\text { QA } \\
\text { Type }\end{array}$} \\
\hline Location & $\begin{array}{c}\text { Collection } \\
\text { Date }\end{array}$ & Result & Units & Result & Units & \\
\hline \multirow[t]{19}{*}{ TRAVEL BLANK } & $4 / 2 / 2007$ & 0.021 & $\mathrm{~Bq} / \mathrm{S}$ & 0.56 & $\mathrm{pCi} / \mathrm{S}$ & Blank \\
\hline & $5 / 7 / 2007$ & $<0.0099$ & $\mathrm{~Bq} / \mathrm{S}$ & $<0.27$ & $\mathrm{pCi} / \mathrm{s}$ & Blank \\
\hline & $5 / 7 / 2007$ & $<0.023$ & $\mathrm{~Bq} / \mathrm{S}$ & $<0.61$ & $\mathrm{pCi} / \mathrm{S}$ & Blank \\
\hline & $6 / 4 / 2007$ & $<0.011$ & $\mathrm{~Bq} / \mathrm{S}$ & $<0.3$ & $\mathrm{pCi} / \mathrm{S}$ & Blank \\
\hline & $6 / 4 / 2007$ & $<0.021$ & $\mathrm{~Bq} / \mathrm{S}$ & $<0.56$ & $\mathrm{pCi} / \mathrm{S}$ & Blank \\
\hline & $7 / 2 / 2007$ & $<0.013$ & $\mathrm{~Bq} / \mathrm{S}$ & $<0.34$ & $\mathrm{pCi} / \mathrm{S}$ & Blank \\
\hline & $7 / 2 / 2007$ & $<0.021$ & $\mathrm{~Bq} / \mathrm{S}$ & $<0.56$ & $\mathrm{pCi} / \mathrm{S}$ & Blank \\
\hline & $8 / 6 / 2007$ & $<0.023$ & $\mathrm{~Bq} / \mathrm{S}$ & $<0.62$ & $\mathrm{pCi} / \mathrm{s}$ & Blank \\
\hline & $8 / 6 / 2007$ & $<0.012$ & $\mathrm{~Bq} / \mathrm{S}$ & $<0.33$ & $\mathrm{pCi} / \mathrm{S}$ & Blank \\
\hline & $9 / 4 / 2007$ & $<0.022$ & $\mathrm{~Bq} / \mathrm{S}$ & $<0.6$ & $\mathrm{pCi} / \mathrm{s}$ & Blank \\
\hline & $9 / 4 / 2007$ & $<0.0084$ & $\mathrm{~Bq} / \mathrm{S}$ & $<0.23$ & $\mathrm{pCi} / \mathrm{S}$ & Blank \\
\hline & $10 / 3 / 2007$ & $<0.0087$ & $\mathrm{~Bq} / \mathrm{S}$ & $<0.23$ & $\mathrm{pCi} / \mathrm{S}$ & Blank \\
\hline & $10 / 3 / 2007$ & $<0.026$ & $\mathrm{~Bq} / \mathrm{S}$ & $<0.71$ & $\mathrm{pCi} / \mathrm{S}$ & Blank \\
\hline & $11 / 5 / 2007$ & $<0.012$ & $\mathrm{~Bq} / \mathrm{S}$ & $<0.31$ & $\mathrm{pCi} / \mathrm{S}$ & Blank \\
\hline & $11 / 5 / 2007$ & $<0.021$ & $\mathrm{~Bq} / \mathrm{S}$ & $<0.58$ & $\mathrm{pCi} / \mathrm{S}$ & Blank \\
\hline & $12 / 3 / 2007$ & $<0.022$ & $\mathrm{~Bq} / \mathrm{S}$ & $<0.6$ & $\mathrm{pCi} / \mathrm{S}$ & Blank \\
\hline & $12 / 3 / 2007$ & $<0.011$ & $\mathrm{~Bq} / \mathrm{S}$ & $<0.29$ & $\mathrm{pCi} / \mathrm{S}$ & Blank \\
\hline & $1 / 7 / 2008$ & $<0.012$ & $\mathrm{~Bq} / \mathrm{S}$ & $<0.32$ & $\mathrm{pCi} / \mathrm{S}$ & Blank \\
\hline & $1 / 7 / 2008$ & $<0.022$ & $\mathrm{~Bq} / \mathrm{S}$ & $<0.59$ & $\mathrm{pCi} / \mathrm{S}$ & Blank \\
\hline
\end{tabular}




\begin{tabular}{|c|c|c|c|c|c|c|}
\hline \multicolumn{2}{|c|}{ Gross beta } & \multicolumn{2}{|c|}{ S.I. } & \multicolumn{2}{|c|}{ Conventional } & \multirow[b]{2}{*}{$\begin{array}{c}\text { QA } \\
\text { Type }\end{array}$} \\
\hline Location & $\begin{array}{c}\text { Collection } \\
\text { Date }\end{array}$ & Result & Units & Result & Units & \\
\hline \multirow[t]{13}{*}{ ENV-44 } & $1 / 9 / 2007$ & 0.00069 & $\mathrm{~Bq} / \mathrm{m}^{3}$ & 0.019 & $\mathrm{pCi} / \mathrm{m}^{3}$ & Sample \\
\hline & $2 / 5 / 2007$ & 0.0012 & $\mathrm{~Bq} / \mathrm{m}^{3}$ & 0.032 & $\mathrm{pCi} / \mathrm{m}^{3}$ & Sample \\
\hline & $3 / 5 / 2007$ & 0.00037 & $\mathrm{~Bq} / \mathrm{m}^{3}$ & 0.01 & $\mathrm{pCi} / \mathrm{m}^{3}$ & Sample \\
\hline & $4 / 2 / 2007$ & 0.00038 & $\mathrm{~Bq} / \mathrm{m}^{3}$ & 0.01 & $\mathrm{pCi} / \mathrm{m}^{3}$ & Sample \\
\hline & $5 / 7 / 2007$ & 0.00028 & $\mathrm{~Bq} / \mathrm{m}^{3}$ & 0.0075 & $\mathrm{pCi} / \mathrm{m}^{3}$ & Sample \\
\hline & $6 / 4 / 2007$ & 0.00024 & $\mathrm{~Bq} / \mathrm{m}^{3}$ & 0.0066 & $\mathrm{pCi} / \mathrm{m}^{3}$ & Sample \\
\hline & $7 / 2 / 2007$ & 0.00018 & $\mathrm{~Bq} / \mathrm{m}^{3}$ & 0.0048 & $\mathrm{pCi} / \mathrm{m}^{3}$ & Sample \\
\hline & $8 / 6 / 2007$ & 0.00015 & $\mathrm{~Bq} / \mathrm{m}^{3}$ & 0.0039 & $\mathrm{pCi} / \mathrm{m}^{3}$ & Sample \\
\hline & $9 / 4 / 2007$ & 0.00026 & $\mathrm{~Bq} / \mathrm{m}^{3}$ & 0.0071 & $\mathrm{pCi} / \mathrm{m}^{3}$ & Sample \\
\hline & $10 / 3 / 2007$ & 0.00054 & $\mathrm{~Bq} / \mathrm{m}^{3}$ & 0.015 & $\mathrm{pCi} / \mathrm{m}^{3}$ & Sample \\
\hline & $11 / 5 / 2007$ & 0.0007 & $\mathrm{~Bq} / \mathrm{m}^{3}$ & 0.019 & $\mathrm{pCi} / \mathrm{m}^{3}$ & Sample \\
\hline & $12 / 3 / 2007$ & 0.00094 & $\mathrm{~Bq} / \mathrm{m}^{3}$ & 0.025 & $\mathrm{pCi} / \mathrm{m}^{3}$ & Sample \\
\hline & $1 / 7 / 2008$ & 0.00044 & $\mathrm{~Bq} / \mathrm{m}^{3}$ & 0.012 & $\mathrm{pCi} / \mathrm{m}^{3}$ & Sample \\
\hline \multirow[t]{13}{*}{ ENV-44-COL } & $1 / 9 / 2007$ & 0.0004 & $\mathrm{~Bq} / \mathrm{m}^{3}$ & 0.011 & $\mathrm{pCi} / \mathrm{m}^{3}$ & Duplicate \\
\hline & $2 / 5 / 2007$ & 0.00081 & $\mathrm{~Bq} / \mathrm{m}^{3}$ & 0.022 & $\mathrm{pCi} / \mathrm{m}^{3}$ & Duplicate \\
\hline & $3 / 5 / 2007$ & 0.00014 & $\mathrm{~Bq} / \mathrm{m}^{3}$ & 0.0039 & $\mathrm{pCi} / \mathrm{m}^{3}$ & Duplicate \\
\hline & $4 / 2 / 2007$ & 0.00036 & $\mathrm{~Bq} / \mathrm{m}^{3}$ & 0.0098 & $\mathrm{pCi} / \mathrm{m}^{3}$ & Duplicate \\
\hline & $5 / 7 / 2007$ & 0.00013 & $\mathrm{~Bq} / \mathrm{m}^{3}$ & 0.0035 & $\mathrm{pCi} / \mathrm{m}^{3}$ & Duplicate \\
\hline & $6 / 4 / 2007$ & 0.00017 & $\mathrm{~Bq} / \mathrm{m}^{3}$ & 0.0045 & $\mathrm{pCi} / \mathrm{m}^{3}$ & Duplicate \\
\hline & $7 / 2 / 2007$ & 0.00011 & $\mathrm{~Bq} / \mathrm{m}^{3}$ & 0.0031 & $\mathrm{pCi} / \mathrm{m}^{3}$ & Duplicate \\
\hline & $8 / 6 / 2007$ & 0.00013 & $\mathrm{~Bq} / \mathrm{m}^{3}$ & 0.0034 & $\mathrm{pCi} / \mathrm{m}^{3}$ & Duplicate \\
\hline & 9/4/2007 & 0.00023 & $\mathrm{~Bq} / \mathrm{m}^{3}$ & 0.0062 & $\mathrm{pCi} / \mathrm{m}^{3}$ & Duplicate \\
\hline & $10 / 3 / 2007$ & 0.00032 & $\mathrm{~Bq} / \mathrm{m}^{3}$ & 0.0088 & $\mathrm{pCi} / \mathrm{m}^{3}$ & Duplicate \\
\hline & $11 / 5 / 2007$ & 0.00026 & $\mathrm{~Bq} / \mathrm{m}^{3}$ & 0.007 & $\mathrm{pCi} / \mathrm{m}^{3}$ & Duplicate \\
\hline & $12 / 3 / 2007$ & 0.00091 & $\mathrm{~Bq} / \mathrm{m}^{3}$ & 0.024 & $\mathrm{pCi} / \mathrm{m}^{3}$ & Duplicate \\
\hline & $1 / 7 / 2008$ & 0.00011 & $\mathrm{~Bq} / \mathrm{m}^{3}$ & 0.0031 & $\mathrm{pCi} / \mathrm{m}^{3}$ & Duplicate \\
\hline \multirow[t]{5}{*}{ ENV-83 } & $1 / 9 / 2007$ & 0.00075 & $\mathrm{~Bq} / \mathrm{m}^{3}$ & 0.02 & $\mathrm{pCi} / \mathrm{m}^{3}$ & Sample \\
\hline & $2 / 5 / 2007$ & 0.0012 & $\mathrm{~Bq} / \mathrm{m}^{3}$ & 0.033 & $\mathrm{pCi} / \mathrm{m}^{3}$ & Sample \\
\hline & $3 / 5 / 2007$ & 0.00038 & $\mathrm{~Bq} / \mathrm{m}^{3}$ & 0.01 & $\mathrm{pCi} / \mathrm{m}^{3}$ & Sample \\
\hline & $4 / 2 / 2007$ & 0.00039 & $\mathrm{~Bq} / \mathrm{m}^{3}$ & 0.011 & $\mathrm{pCi} / \mathrm{m}^{3}$ & Sample \\
\hline & $5 / 7 / 2007$ & 0.0003 & $\mathrm{~Bq} / \mathrm{m}^{3}$ & 0.0082 & $\mathrm{pCi} / \mathrm{m}^{3}$ & Sample \\
\hline
\end{tabular}




\begin{tabular}{|c|c|c|c|c|c|c|}
\hline \multicolumn{2}{|c|}{ Gross beta } & \multicolumn{2}{|c|}{ S.I. } & \multicolumn{2}{|c|}{ Conventional } & \multirow[b]{2}{*}{$\begin{array}{c}\text { QA } \\
\text { Type }\end{array}$} \\
\hline Location & $\begin{array}{c}\text { Collection } \\
\text { Date }\end{array}$ & Result & Units & Result & Units & \\
\hline \multirow[t]{8}{*}{ ENV-83 } & $6 / 4 / 2007$ & 0.00027 & $\mathrm{~Bq} / \mathrm{m}^{3}$ & 0.0074 & $\mathrm{pCi} / \mathrm{m}^{3}$ & Sample \\
\hline & $7 / 2 / 2007$ & 0.00017 & $\mathrm{~Bq} / \mathrm{m}^{3}$ & 0.0047 & $\mathrm{pCi} / \mathrm{m}^{3}$ & Sample \\
\hline & $8 / 6 / 2007$ & 0.00017 & $\mathrm{~Bq} / \mathrm{m}^{3}$ & 0.0046 & $\mathrm{pCi} / \mathrm{m}^{3}$ & Sample \\
\hline & 9/4/2007 & 0.00036 & $\mathrm{~Bq} / \mathrm{m}^{3}$ & 0.0096 & $\mathrm{pCi} / \mathrm{m}^{3}$ & Sample \\
\hline & $10 / 3 / 2007$ & 0.00061 & $\mathrm{~Bq} / \mathrm{m}^{3}$ & 0.017 & $\mathrm{pCi} / \mathrm{m}^{3}$ & Sample \\
\hline & $11 / 5 / 2007$ & 0.00076 & $\mathrm{~Bq} / \mathrm{m}^{3}$ & 0.021 & $\mathrm{pCi} / \mathrm{m}^{3}$ & Sample \\
\hline & $12 / 3 / 2007$ & 0.00092 & $\mathrm{~Bq} / \mathrm{m}^{3}$ & 0.025 & $\mathrm{pCi} / \mathrm{m}^{3}$ & Sample \\
\hline & $1 / 7 / 2008$ & 0.00043 & $\mathrm{~Bq} / \mathrm{m}^{3}$ & 0.012 & $\mathrm{pCi} / \mathrm{m}^{3}$ & Sample \\
\hline \multirow[t]{13}{*}{ ENV-B13A } & $1 / 9 / 2007$ & 0.00061 & $\mathrm{~Bq} / \mathrm{m}^{3}$ & 0.017 & $\mathrm{pCi} / \mathrm{m}^{3}$ & Sample \\
\hline & $2 / 5 / 2007$ & 0.00083 & $\mathrm{~Bq} / \mathrm{m}^{3}$ & 0.022 & $\mathrm{pCi} / \mathrm{m}^{3}$ & Sample \\
\hline & $3 / 5 / 2007$ & 0.0004 & $\mathrm{~Bq} / \mathrm{m}^{3}$ & 0.011 & $\mathrm{pCi} / \mathrm{m}^{3}$ & Sample \\
\hline & 4/2/2007 & 0.00039 & $\mathrm{~Bq} / \mathrm{m}^{3}$ & 0.01 & $\mathrm{pCi} / \mathrm{m}^{3}$ & Sample \\
\hline & $5 / 7 / 2007$ & 0.00032 & $\mathrm{~Bq} / \mathrm{m}^{3}$ & 0.0086 & $\mathrm{pCi} / \mathrm{m}^{3}$ & Sample \\
\hline & $6 / 4 / 2007$ & 0.00027 & $\mathrm{~Bq} / \mathrm{m}^{3}$ & 0.0073 & $\mathrm{pCi} / \mathrm{m}^{3}$ & Sample \\
\hline & $7 / 2 / 2007$ & 0.00017 & $\mathrm{~Bq} / \mathrm{m}^{3}$ & 0.0046 & $\mathrm{pCi} / \mathrm{m}^{3}$ & Sample \\
\hline & $8 / 6 / 2007$ & 0.00014 & $\mathrm{~Bq} / \mathrm{m}^{3}$ & 0.0037 & $\mathrm{pCi} / \mathrm{m}^{3}$ & Sample \\
\hline & 9/4/2007 & 0.00031 & $\mathrm{~Bq} / \mathrm{m}^{3}$ & 0.0083 & $\mathrm{pCi} / \mathrm{m}^{3}$ & Sample \\
\hline & $10 / 3 / 2007$ & 0.00053 & $\mathrm{~Bq} / \mathrm{m}^{3}$ & 0.014 & $\mathrm{pCi} / \mathrm{m}^{3}$ & Sample \\
\hline & $11 / 5 / 2007$ & 0.00074 & $\mathrm{~Bq} / \mathrm{m}^{3}$ & 0.02 & $\mathrm{pCi} / \mathrm{m}^{3}$ & Sample \\
\hline & $12 / 3 / 2007$ & 0.00099 & $\mathrm{~Bq} / \mathrm{m}^{3}$ & 0.027 & $\mathrm{pCi} / \mathrm{m}^{3}$ & Sample \\
\hline & $1 / 7 / 2008$ & 0.00045 & $\mathrm{~Bq} / \mathrm{m}^{3}$ & 0.012 & $\mathrm{pCi} / \mathrm{m}^{3}$ & Sample \\
\hline \multirow[t]{11}{*}{ ENV-B13C } & $1 / 9 / 2007$ & 0.0007 & $\mathrm{~Bq} / \mathrm{m}^{3}$ & 0.019 & $\mathrm{pCi} / \mathrm{m}^{3}$ & Sample \\
\hline & $2 / 5 / 2007$ & 0.0013 & $\mathrm{~Bq} / \mathrm{m}^{3}$ & 0.036 & $\mathrm{pCi} / \mathrm{m}^{3}$ & Sample \\
\hline & $3 / 5 / 2007$ & 0.00042 & $\mathrm{~Bq} / \mathrm{m}^{3}$ & 0.011 & $\mathrm{pCi} / \mathrm{m}^{3}$ & Sample \\
\hline & 4/2/2007 & 0.00041 & $\mathrm{~Bq} / \mathrm{m}^{3}$ & 0.011 & $\mathrm{pCi} / \mathrm{m}^{3}$ & Sample \\
\hline & $5 / 7 / 2007$ & 0.00029 & $\mathrm{~Bq} / \mathrm{m}^{3}$ & 0.0079 & $\mathrm{pCi} / \mathrm{m}^{3}$ & Sample \\
\hline & $6 / 4 / 2007$ & 0.00032 & $\mathrm{~Bq} / \mathrm{m}^{3}$ & 0.0086 & $\mathrm{pCi} / \mathrm{m}^{3}$ & Sample \\
\hline & $7 / 2 / 2007$ & 0.00018 & $\mathrm{~Bq} / \mathrm{m}^{3}$ & 0.005 & $\mathrm{pCi} / \mathrm{m}^{3}$ & Sample \\
\hline & $8 / 6 / 2007$ & 0.00016 & $\mathrm{~Bq} / \mathrm{m}^{3}$ & 0.0043 & $\mathrm{pCi} / \mathrm{m}^{3}$ & Sample \\
\hline & $9 / 4 / 2007$ & 0.00034 & $\mathrm{~Bq} / \mathrm{m}^{3}$ & 0.0093 & $\mathrm{pCi} / \mathrm{m}^{3}$ & Sample \\
\hline & $10 / 3 / 2007$ & 0.0005 & $\mathrm{~Bq} / \mathrm{m}^{3}$ & 0.014 & $\mathrm{pCi} / \mathrm{m}^{3}$ & Sample \\
\hline & $11 / 5 / 2007$ & 0.0007 & $\mathrm{~Bq} / \mathrm{m}^{3}$ & 0.019 & $\mathrm{pCi} / \mathrm{m}^{3}$ & Sample \\
\hline
\end{tabular}




\begin{tabular}{|c|c|c|c|c|c|c|}
\hline \multicolumn{2}{|c|}{ Gross beta } & \multicolumn{2}{|c|}{ S.I. } & \multicolumn{2}{|c|}{ Conventional } & \multirow[b]{2}{*}{$\begin{array}{c}\text { QA } \\
\text { Type }\end{array}$} \\
\hline Location & $\begin{array}{c}\text { Collection } \\
\text { Date }\end{array}$ & Result & Units & Result & Units & \\
\hline \multirow[t]{2}{*}{ ENV-B13C } & $12 / 3 / 2007$ & 0.0009 & $\mathrm{~Bq} / \mathrm{m}^{3}$ & 0.024 & $\mathrm{pCi} / \mathrm{m}^{3}$ & Sample \\
\hline & $1 / 7 / 2008$ & 0.00046 & $\mathrm{~Bq} / \mathrm{m}^{3}$ & 0.012 & $\mathrm{pCi} / \mathrm{m}^{3}$ & Sample \\
\hline \multirow[t]{26}{*}{ LOT BLANK } & $1 / 9 / 2007$ & $<0.019$ & $\mathrm{~Bq} / \mathrm{S}$ & $<0.5$ & $\mathrm{pCi} / \mathrm{S}$ & Blank \\
\hline & $1 / 9 / 2007$ & $<0.036$ & $\mathrm{~Bq} / \mathrm{S}$ & $<0.96$ & $\mathrm{pCi} / \mathrm{S}$ & Blank \\
\hline & 2/5/2007 & $<0.032$ & $\mathrm{~Bq} / \mathrm{S}$ & $<0.87$ & $\mathrm{pCi} / \mathrm{S}$ & Blank \\
\hline & $2 / 5 / 2007$ & $<0.019$ & $\mathrm{~Bq} / \mathrm{S}$ & $<0.5$ & $\mathrm{pCi} / \mathrm{S}$ & Blank \\
\hline & $3 / 5 / 2007$ & $<0.036$ & $\mathrm{~Bq} / \mathrm{S}$ & $<0.96$ & $\mathrm{pCi} / \mathrm{S}$ & Blank \\
\hline & $3 / 5 / 2007$ & $<0.017$ & $\mathrm{~Bq} / \mathrm{S}$ & $<0.47$ & $\mathrm{pCi} / \mathrm{S}$ & Blank \\
\hline & $4 / 2 / 2007$ & $<0.02$ & $\mathrm{~Bq} / \mathrm{S}$ & $<0.55$ & $\mathrm{pCi} / \mathrm{S}$ & Blank \\
\hline & 4/2/2007 & 0.048 & $\mathrm{~Bq} / \mathrm{S}$ & 1.3 & $\mathrm{pCi} / \mathrm{S}$ & Blank \\
\hline & $5 / 7 / 2007$ & $<0.033$ & $\mathrm{~Bq} / \mathrm{S}$ & $<0.88$ & $\mathrm{pCi} / \mathrm{S}$ & Blank \\
\hline & $5 / 7 / 2007$ & 0.032 & $\mathrm{~Bq} / \mathrm{S}$ & 0.86 & $\mathrm{pCi} / \mathrm{S}$ & Blank \\
\hline & $6 / 4 / 2007$ & $<0.024$ & $\mathrm{~Bq} / \mathrm{S}$ & $<0.66$ & $\mathrm{pCi} / \mathrm{S}$ & Blank \\
\hline & $6 / 4 / 2007$ & $<0.033$ & $\mathrm{~Bq} / \mathrm{S}$ & $<0.88$ & $\mathrm{pCi} / \mathrm{S}$ & Blank \\
\hline & $7 / 2 / 2007$ & $<0.036$ & $\mathrm{~Bq} / \mathrm{S}$ & $<0.96$ & $\mathrm{pCi} / \mathrm{S}$ & Blank \\
\hline & $7 / 2 / 2007$ & $<0.026$ & $\mathrm{~Bq} / \mathrm{S}$ & $<0.71$ & $\mathrm{pCi} / \mathrm{S}$ & Blank \\
\hline & $8 / 6 / 2007$ & 0.034 & $\mathrm{~Bq} / \mathrm{S}$ & 0.92 & $\mathrm{pCi} / \mathrm{S}$ & Blank \\
\hline & $8 / 6 / 2007$ & $<0.036$ & $\mathrm{~Bq} / \mathrm{S}$ & $<0.96$ & $\mathrm{pCi} / \mathrm{S}$ & Blank \\
\hline & $9 / 4 / 2007$ & $<0.031$ & $\mathrm{~Bq} / \mathrm{S}$ & $<0.85$ & $\mathrm{pCi} / \mathrm{S}$ & Blank \\
\hline & 9/4/2007 & 0.081 & $\mathrm{~Bq} / \mathrm{S}$ & 2.2 & $\mathrm{pCi} / \mathrm{S}$ & Blank \\
\hline & $10 / 3 / 2007$ & $<0.025$ & $\mathrm{~Bq} / \mathrm{S}$ & $<0.68$ & $\mathrm{pCi} / \mathrm{S}$ & Blank \\
\hline & $10 / 3 / 2007$ & $<0.04$ & $\mathrm{~Bq} / \mathrm{S}$ & $<1.1$ & $\mathrm{pCi} / \mathrm{S}$ & Blank \\
\hline & $11 / 5 / 2007$ & $<0.036$ & $\mathrm{~Bq} / \mathrm{S}$ & $<0.96$ & $\mathrm{pCi} / \mathrm{S}$ & Blank \\
\hline & $11 / 5 / 2007$ & 0.082 & $\mathrm{~Bq} / \mathrm{S}$ & 2.2 & $\mathrm{pCi} / \mathrm{S}$ & Blank \\
\hline & $12 / 3 / 2007$ & $<0.04$ & $\mathrm{~Bq} / \mathrm{S}$ & $<1.1$ & $\mathrm{pCi} / \mathrm{S}$ & Blank \\
\hline & $12 / 3 / 2007$ & $<0.012$ & $\mathrm{~Bq} / \mathrm{S}$ & $<0.33$ & $\mathrm{pCi} / \mathrm{S}$ & Blank \\
\hline & $1 / 7 / 2008$ & $<0.035$ & $\mathrm{~Bq} / \mathrm{S}$ & $<0.94$ & $\mathrm{pCi} / \mathrm{S}$ & Blank \\
\hline & $1 / 7 / 2008$ & 0.064 & $\mathrm{~Bq} / \mathrm{S}$ & 1.7 & $\mathrm{pCi} / \mathrm{S}$ & Blank \\
\hline \multirow[t]{7}{*}{ TRAVEL BLANK } & $1 / 9 / 2007$ & $<0.036$ & $\mathrm{~Bq} / \mathrm{S}$ & $<0.97$ & $\mathrm{pCi} / \mathrm{S}$ & Blank \\
\hline & $1 / 9 / 2007$ & $<0.01$ & $\mathrm{~Bq} / \mathrm{S}$ & $<0.27$ & $\mathrm{pCi} / \mathrm{S}$ & Blank \\
\hline & $2 / 5 / 2007$ & $<0.02$ & $\mathrm{~Bq} / \mathrm{S}$ & $<0.54$ & $\mathrm{pCi} / \mathrm{S}$ & Blank \\
\hline & $2 / 5 / 2007$ & 0.033 & $\mathrm{~Bq} / \mathrm{S}$ & 0.89 & $\mathrm{pCi} / \mathrm{S}$ & Blank \\
\hline & $3 / 5 / 2007$ & $<0.021$ & $\mathrm{~Bq} / \mathrm{S}$ & $<0.56$ & $\mathrm{pCi} / \mathrm{S}$ & Blank \\
\hline & $3 / 5 / 2007$ & $<0.036$ & $\mathrm{~Bq} / \mathrm{S}$ & $<0.98$ & pCi/s & Blank \\
\hline & 4/2/2007 & $<0.022$ & $\mathrm{~Bq} / \mathrm{S}$ & $<0.6$ & $\mathrm{pCi} / \mathrm{S}$ & Blank \\
\hline
\end{tabular}




\begin{tabular}{|l|c|c|c|c|c|c|}
\hline \multicolumn{2}{|c|}{ Gross beta } & \multicolumn{2}{c|}{ S.I. } & \multicolumn{2}{c|}{ Conventional } & \\
\hline Location & $\begin{array}{c}\text { Collection } \\
\text { Date }\end{array}$ & Result & Units & Result & Units & $\begin{array}{c}\text { QA } \\
\text { Type }\end{array}$ \\
\hline TRAVEL BLANK & $4 / 2 / 2007$ & 0.044 & $\mathrm{~Bq} / \mathrm{S}$ & 1.2 & $\mathrm{pCi} / \mathrm{S}$ & Blank \\
\hline & $5 / 7 / 2007$ & $<0.033$ & $\mathrm{~Bq} / \mathrm{S}$ & $<0.89$ & $\mathrm{pCi} / \mathrm{S}$ & Blank \\
\hline & $5 / 7 / 2007$ & 0.045 & $\mathrm{~Bq} / \mathrm{S}$ & 1.2 & $\mathrm{pCi} / \mathrm{S}$ & Blank \\
\hline & $6 / 4 / 2007$ & $<0.023$ & $\mathrm{~Bq} / \mathrm{S}$ & $<0.62$ & $\mathrm{pCi} / \mathrm{S}$ & Blank \\
\hline & $6 / 4 / 2007$ & $<0.033$ & $\mathrm{~Bq} / \mathrm{S}$ & $<0.89$ & $\mathrm{pCi} / \mathrm{S}$ & Blank \\
\hline & $7 / 2 / 2007$ & $<0.036$ & $\mathrm{~Bq} / \mathrm{S}$ & $<0.98$ & $\mathrm{pCi} / \mathrm{S}$ & Blank \\
\hline & $7 / 2 / 2007$ & $<0.025$ & $\mathrm{~Bq} / \mathrm{S}$ & $<0.69$ & $\mathrm{pCi} / \mathrm{S}$ & Blank \\
\hline & $8 / 6 / 2007$ & $<0.036$ & $\mathrm{~Bq} / \mathrm{S}$ & $<0.96$ & $\mathrm{pCi} / \mathrm{S}$ & Blank \\
\hline & $8 / 6 / 2007$ & 0.035 & $\mathrm{~Bq} / \mathrm{S}$ & 0.94 & $\mathrm{pCi} / \mathrm{S}$ & Blank \\
\hline & $9 / 4 / 2007$ & 0.076 & $\mathrm{~Bq} / \mathrm{S}$ & 2 & $\mathrm{pCi} / \mathrm{S}$ & Blank \\
\hline & $9 / 4 / 2007$ & $<0.031$ & $\mathrm{~Bq} / \mathrm{S}$ & $<0.83$ & $\mathrm{pCi} / \mathrm{S}$ & Blank \\
\hline & $10 / 3 / 2007$ & 0.032 & $\mathrm{~Bq} / \mathrm{S}$ & 0.87 & $\mathrm{pCi} / \mathrm{S}$ & Blank \\
\hline & $10 / 3 / 2007$ & $<0.04$ & $\mathrm{~Bq} / \mathrm{S}$ & $<1.1$ & $\mathrm{pCi} / \mathrm{S}$ & Blank \\
\hline & $11 / 5 / 2007$ & 0.061 & $\mathrm{~Bq} / \mathrm{S}$ & 1.6 & $\mathrm{pCi} / \mathrm{S}$ & Blank \\
\hline & $11 / 5 / 2007$ & $<0.035$ & $\mathrm{~Bq} / \mathrm{S}$ & $<0.94$ & $\mathrm{pCi} / \mathrm{S}$ & Blank \\
\hline & $12 / 3 / 2007$ & $<0.021$ & $\mathrm{~Bq} / \mathrm{S}$ & $<0.57$ & $\mathrm{pCi} / \mathrm{S}$ & Blank \\
\hline & $12 / 3 / 2007$ & $<0.04$ & $\mathrm{~Bq} / \mathrm{S}$ & $<1.1$ & $\mathrm{pCi} / \mathrm{S}$ & Blank \\
\hline & $1 / 7 / 2008$ & 0.063 & $\mathrm{~Bq} / \mathrm{S}$ & 1.7 & $\mathrm{pCi} / \mathrm{S}$ & Blank \\
\hline & $1 / 7 / 2008$ & $<0.035$ & $\mathrm{~Bq} / \mathrm{S}$ & $<0.94$ & $\mathrm{pCi} / \mathrm{S}$ & Blank \\
\hline
\end{tabular}




\begin{tabular}{|c|c|c|c|c|c|c|c|}
\hline \multicolumn{2}{|c|}{ Radiological Activity } & \multirow[b]{2}{*}{$\begin{array}{c}\text { Collection } \\
\text { Date }\end{array}$} & \multicolumn{2}{|c|}{ S.I. } & \multicolumn{2}{|c|}{ Conventional } & \multirow[b]{2}{*}{$\begin{array}{l}\text { QA } \\
\text { Type }\end{array}$} \\
\hline Analyte & Location & & Result & Units & Result & Units & \\
\hline \multirow[t]{12}{*}{ Gross alpha } & ENV-44 & $10 / 29 / 2007$ & $<0.016$ & $\mathrm{~Bq} / \mathrm{L}$ & $<0.42$ & $\mathrm{pCi} / \mathrm{L}$ & Sample \\
\hline & & $10 / 29 / 2007$ & $<0.046$ & $\mathrm{~Bq} / \mathrm{L}$ & $<1.2$ & $\mathrm{pCi} / \mathrm{L}$ & Split \\
\hline & & $11 / 28 / 2007$ & $<0.047$ & $\mathrm{~Bq} / \mathrm{L}$ & $<1.3$ & pCi/L & Sample \\
\hline & & $12 / 19 / 2007$ & $<0.052$ & $\mathrm{~Bq} / \mathrm{L}$ & $<1.4$ & $\mathrm{pCi} / \mathrm{L}$ & Sample \\
\hline & ENV-75 & $1 / 30 / 2007$ & $<0.046$ & $\mathrm{~Bq} / \mathrm{L}$ & $<1.2$ & $\mathrm{pCi} / \mathrm{L}$ & Sample \\
\hline & & $1 / 30 / 2007$ & $<0.037$ & $\mathrm{~Bq} / \mathrm{L}$ & $<1$ & pCill & Split \\
\hline & & 2/28/2007 & $<0.04$ & $\mathrm{~Bq} / \mathrm{L}$ & $<1.1$ & pCi/L & Sample \\
\hline & & $3 / 28 / 2007$ & $<0.045$ & $\mathrm{~Bq} / \mathrm{L}$ & $<1.2$ & $\mathrm{pCi} / \mathrm{L}$ & Sample \\
\hline & & $4 / 25 / 2007$ & $<0.053$ & $\mathrm{~Bq} / \mathrm{L}$ & $<1.4$ & pCi/L & Sample \\
\hline & & $5 / 30 / 2007$ & $<0.052$ & $\mathrm{~Bq} / \mathrm{L}$ & $<1.4$ & pCi/L & Sample \\
\hline & FIELD BLANK & $1 / 30 / 2007$ & $<0.04$ & $\mathrm{~Bq} / \mathrm{L}$ & $<1.1$ & $\mathrm{pCi} / \mathrm{L}$ & Blank \\
\hline & & 1/30/2007 & $<0.039$ & $\mathrm{~Bq} / \mathrm{L}$ & $<1$ & pCi/L & Blank \\
\hline \multirow[t]{12}{*}{ Gross beta } & ENV-44 & 10/29/2007 & 0.073 & $\mathrm{~Bq} / \mathrm{L}$ & 2 & $\mathrm{pCi} / \mathrm{L}$ & Sample \\
\hline & & $10 / 29 / 2007$ & $<0.078$ & $\mathrm{~Bq} / \mathrm{L}$ & $<2.1$ & $\mathrm{pCi} / \mathrm{L}$ & Split \\
\hline & & $11 / 28 / 2007$ & $<0.11$ & $\mathrm{~Bq} / \mathrm{L}$ & $<2.9$ & pCi/L & Sample \\
\hline & & $12 / 19 / 2007$ & $<0.08$ & $\mathrm{~Bq} / \mathrm{L}$ & $<2.2$ & $\mathrm{pCi} / \mathrm{L}$ & Sample \\
\hline & ENV-75 & $1 / 30 / 2007$ & $<0.092$ & $\mathrm{~Bq} / \mathrm{L}$ & $<2.5$ & $\mathrm{pCi} / \mathrm{L}$ & Sample \\
\hline & & 1/30/2007 & $<0.078$ & $\mathrm{~Bq} / \mathrm{L}$ & $<2.1$ & pCi/L & Split \\
\hline & & $2 / 28 / 2007$ & $<0.082$ & $\mathrm{~Bq} / \mathrm{L}$ & $<2.2$ & $\mathrm{pCi} / \mathrm{L}$ & Sample \\
\hline & & $3 / 28 / 2007$ & 0.13 & $\mathrm{~Bq} / \mathrm{L}$ & 3.5 & $\mathrm{pCi} / \mathrm{L}$ & Sample \\
\hline & & 4/25/2007 & 0.11 & $\mathrm{~Bq} / \mathrm{L}$ & 3 & pCi/L & Sample \\
\hline & & $5 / 30 / 2007$ & 0.13 & $\mathrm{~Bq} / \mathrm{L}$ & 3.6 & $\mathrm{pCi} / \mathrm{L}$ & Sample \\
\hline & FIELD BLANK & $1 / 30 / 2007$ & $<0.085$ & $\mathrm{~Bq} / \mathrm{L}$ & $<2.3$ & pCi/L & Blank \\
\hline & & $1 / 30 / 2007$ & 0.69 & $\mathrm{~Bq} / \mathrm{L}$ & 18 & pCi/L & Blank \\
\hline \multirow[t]{10}{*}{ Tritium } & ENV-44 & $10 / 29 / 2007$ & $<6$ & $\mathrm{~Bq} / \mathrm{L}$ & $<160$ & $\mathrm{pCi} / \mathrm{L}$ & Sample \\
\hline & & $10 / 29 / 2007$ & $<9.6$ & $\mathrm{~Bq} / \mathrm{L}$ & $<260$ & pCi/L & Split \\
\hline & & $11 / 28 / 2007$ & $<5.7$ & $\mathrm{~Bq} / \mathrm{L}$ & $<160$ & pCi/L & Sample \\
\hline & & $12 / 19 / 2007$ & $<6.1$ & $\mathrm{~Bq} / \mathrm{L}$ & $<160$ & pCi/L & Sample \\
\hline & ENV-75 & $1 / 30 / 2007$ & $<6.5$ & $\mathrm{~Bq} / \mathrm{L}$ & $<180$ & $\mathrm{pCi} / \mathrm{L}$ & Sample \\
\hline & & $1 / 30 / 2007$ & $<10$ & $\mathrm{~Bq} / \mathrm{L}$ & $<280$ & pCi/L & Split \\
\hline & & 2/28/2007 & $<5.5$ & $\mathrm{~Bq} / \mathrm{L}$ & $<150$ & $\mathrm{pCi} / \mathrm{L}$ & Sample \\
\hline & & $3 / 28 / 2007$ & $<6.5$ & $\mathrm{~Bq} / \mathrm{L}$ & $<180$ & pCi/L & Sample \\
\hline & & $4 / 25 / 2007$ & $<6.3$ & $\mathrm{~Bq} / \mathrm{L}$ & $<170$ & pCi/L & Sample \\
\hline & & $5 / 30 / 2007$ & $<6.1$ & $\mathrm{~Bq} / \mathrm{L}$ & $<160$ & $\mathrm{pCi} / \mathrm{L}$ & Sample \\
\hline
\end{tabular}




\begin{tabular}{|l|c|c|c|c|c|c|c|}
\hline \multicolumn{2}{|c|}{ Radiological Activity } & \multicolumn{2}{c|}{ S.I. } & \multicolumn{2}{c|}{ Conventional } & \\
\hline Analyte & Location & $\begin{array}{c}\text { Collection } \\
\text { Date }\end{array}$ & Result & Units & Result & Units & $\begin{array}{c}\text { QA } \\
\text { Type }\end{array}$ \\
\hline Tritium & FIELD BLANK & $1 / 30 / 2007$ & 11 & $\mathrm{~Bq} / \mathrm{L}$ & 290 & $\mathrm{pCi} / \mathrm{L}$ & Blank \\
\hline & & $1 / 30 / 2007$ & $<6.5$ & $\mathrm{~Bq} / \mathrm{L}$ & $<180$ & $\mathrm{pC} / \mathrm{L}$ & $\mathrm{Blank}$ \\
\hline
\end{tabular}




\begin{tabular}{|c|c|c|c|c|c|c|c|}
\hline \multicolumn{2}{|c|}{ Radiological Activity } & \multirow[b]{2}{*}{$\begin{array}{l}\text { Collection } \\
\text { Date }\end{array}$} & \multicolumn{2}{|c|}{ S.I. } & \multicolumn{2}{|c|}{ Conventional } & \multirow[b]{2}{*}{$\begin{array}{c}\text { QA } \\
\text { Type }\end{array}$} \\
\hline Analyte & Location & & Result & Units & Result & Units & \\
\hline \multirow[t]{16}{*}{ Gross alpha } & Chicken Creek & 2/21/2007 & $<0.066$ & $\mathrm{~Bq} / \mathrm{L}$ & $<1.8$ & pCi/L & Sample \\
\hline & & 2/21/2007 & 0.056 & $\mathrm{~Bq} / \mathrm{L}$ & 1.5 & pCi/L & Split \\
\hline & & $5 / 15 / 2007$ & $<0.073$ & $\mathrm{~Bq} / \mathrm{L}$ & $<2$ & pCi/L & Sample \\
\hline & & $8 / 21 / 2007$ & $<0.07$ & $\mathrm{~Bq} / \mathrm{L}$ & $<1.9$ & $\mathrm{pCi} / \mathrm{L}$ & Sample \\
\hline & & $11 / 19 / 2007$ & $<0.071$ & $\mathrm{~Bq} / \mathrm{L}$ & $<1.9$ & $\mathrm{pCi} / \mathrm{L}$ & Sample \\
\hline & FIELD BLANK & $2 / 21 / 2007$ & $<0.032$ & $\mathrm{~Bq} / \mathrm{L}$ & $<0.86$ & $\mathrm{pCi} / \mathrm{L}$ & Blank \\
\hline & & 2/21/2007 & $<0.026$ & $\mathrm{~Bq} / \mathrm{L}$ & $<0.7$ & pCi/L & Blank \\
\hline & $\begin{array}{l}\text { N. Fork Strawberry } \\
\text { Creek }\end{array}$ & $2 / 21 / 2007$ & $<0.073$ & $\mathrm{~Bq} / \mathrm{L}$ & $<2$ & pCi/L & Sample \\
\hline & & $5 / 15 / 2007$ & 0.099 & $\mathrm{~Bq} / \mathrm{L}$ & 2.7 & $\mathrm{pCi} / \mathrm{L}$ & Sample \\
\hline & & $8 / 21 / 2007$ & $<0.07$ & $\mathrm{~Bq} / \mathrm{L}$ & $<1.9$ & $\mathrm{pCi} / \mathrm{L}$ & Sample \\
\hline & & $8 / 21 / 2007$ & $<0.048$ & $\mathrm{~Bq} / \mathrm{L}$ & $<1.3$ & $\mathrm{pCi} / \mathrm{L}$ & Split \\
\hline & & $11 / 19 / 2007$ & 0.074 & $\mathrm{~Bq} / \mathrm{L}$ & 2 & $\mathrm{pCi} / \mathrm{L}$ & Sample \\
\hline & Strawberry Creek (UC) & 2/21/2007 & $<0.071$ & $\mathrm{~Bq} / \mathrm{L}$ & $<1.9$ & $\mathrm{pCi} / \mathrm{L}$ & Sample \\
\hline & & $5 / 15 / 2007$ & 0.13 & $\mathrm{~Bq} / \mathrm{L}$ & 3.6 & $\mathrm{pCi} / \mathrm{L}$ & Sample \\
\hline & & $8 / 21 / 2007$ & $<0.07$ & $\mathrm{~Bq} / \mathrm{L}$ & $<1.9$ & $\mathrm{pCi} / \mathrm{L}$ & Sample \\
\hline & & $11 / 19 / 2007$ & $<0.05$ & $\mathrm{~Bq} / \mathrm{L}$ & $<1.4$ & $\mathrm{pCi} / \mathrm{L}$ & Sample \\
\hline \multirow[t]{16}{*}{ Gross beta } & Chicken Creek & 2/21/2007 & $<0.086$ & $\mathrm{~Bq} / \mathrm{L}$ & $<2.3$ & pCi/L & Sample \\
\hline & & 2/21/2007 & $<0.07$ & $\mathrm{~Bq} / \mathrm{L}$ & $<1.9$ & $\mathrm{pCi} / \mathrm{L}$ & Split \\
\hline & & $5 / 15 / 2007$ & $<0.11$ & $\mathrm{~Bq} / \mathrm{L}$ & $<2.9$ & pCi/L & Sample \\
\hline & & $8 / 21 / 2007$ & $<0.083$ & $\mathrm{~Bq} / \mathrm{L}$ & $<2.2$ & $\mathrm{pCi} / \mathrm{L}$ & Sample \\
\hline & & $11 / 19 / 2007$ & $<0.053$ & $\mathrm{~Bq} / \mathrm{L}$ & $<1.4$ & $\mathrm{pCi} / \mathrm{L}$ & Sample \\
\hline & FIELD BLANK & $2 / 21 / 2007$ & $<0.083$ & $\mathrm{~Bq} / \mathrm{L}$ & $<2.2$ & $\mathrm{pCi} / \mathrm{L}$ & Blank \\
\hline & & 2/21/2007 & $<0.054$ & $\mathrm{~Bq} / \mathrm{L}$ & $<1.4$ & $\mathrm{pCi} / \mathrm{L}$ & Blank \\
\hline & $\begin{array}{l}\text { N. Fork Strawberry } \\
\text { Creek }\end{array}$ & $2 / 21 / 2007$ & $<0.091$ & $\mathrm{~Bq} / \mathrm{L}$ & $<2.4$ & $\mathrm{pCi} / \mathrm{L}$ & Sample \\
\hline & & $5 / 15 / 2007$ & $<0.092$ & $\mathrm{~Bq} / \mathrm{L}$ & $<2.5$ & $\mathrm{pCi} / \mathrm{L}$ & Sample \\
\hline & & $8 / 21 / 2007$ & $<0.083$ & $\mathrm{~Bq} / \mathrm{L}$ & $<2.2$ & $\mathrm{pCi} / \mathrm{L}$ & Sample \\
\hline & & $8 / 21 / 2007$ & $<0.074$ & $\mathrm{~Bq} / \mathrm{L}$ & $<2$ & $\mathrm{pCi} / \mathrm{L}$ & Split \\
\hline & & $11 / 19 / 2007$ & $<0.037$ & $\mathrm{~Bq} / \mathrm{L}$ & $<1$ & $\mathrm{pCi} / \mathrm{L}$ & Sample \\
\hline & Strawberry Creek (UC) & 2/21/2007 & $<0.08$ & $\mathrm{~Bq} / \mathrm{L}$ & $<2.2$ & $\mathrm{pCi} / \mathrm{L}$ & Sample \\
\hline & & $5 / 15 / 2007$ & 0.16 & $\mathrm{~Bq} / \mathrm{L}$ & 4.4 & $\mathrm{pCi} / \mathrm{L}$ & Sample \\
\hline & & $8 / 21 / 2007$ & 0.11 & $\mathrm{~Bq} / \mathrm{L}$ & 3.1 & $\mathrm{pCi} / \mathrm{L}$ & Sample \\
\hline & & $11 / 19 / 2007$ & 0.058 & $\mathrm{~Bq} / \mathrm{L}$ & 1.6 & $\mathrm{pCi} / \mathrm{L}$ & Sample \\
\hline
\end{tabular}




\begin{tabular}{|c|c|c|c|c|c|c|c|}
\hline \multicolumn{2}{|c|}{ Radiological Activity } & \multirow[b]{2}{*}{$\begin{array}{c}\text { Collection } \\
\text { Date }\end{array}$} & \multicolumn{2}{|c|}{ S.I. } & \multicolumn{2}{|c|}{ Conventional } & \multirow[b]{2}{*}{$\begin{array}{c}\text { QA } \\
\text { Type }\end{array}$} \\
\hline Analyte & Location & & Result & Units & Result & Units & \\
\hline \multirow[t]{21}{*}{ Tritium } & Chicken Creek & $2 / 21 / 2007$ & 12 & $\mathrm{~Bq} / \mathrm{L}$ & 320 & $\mathrm{pCi} / \mathrm{L}$ & Sample \\
\hline & & $2 / 21 / 2007$ & 11 & $\mathrm{~Bq} / \mathrm{L}$ & 300 & $\mathrm{pCi} / \mathrm{L}$ & Split \\
\hline & & $5 / 15 / 2007$ & $<6.5$ & $\mathrm{~Bq} / \mathrm{L}$ & $<180$ & $\mathrm{pCi} / \mathrm{L}$ & Sample \\
\hline & & $8 / 21 / 2007$ & $<5.9$ & $\mathrm{~Bq} / \mathrm{L}$ & $<160$ & pCi/L & Sample \\
\hline & & $11 / 19 / 2007$ & $<6.1$ & $\mathrm{~Bq} / \mathrm{L}$ & $<170$ & $\mathrm{pCi} / \mathrm{L}$ & Sample \\
\hline & $\begin{array}{l}\text { Chicken Creek } \\
\text { Downstream }\end{array}$ & $3 / 1 / 2007$ & 9.3 & $\mathrm{~Bq} / \mathrm{L}$ & 250 & $\mathrm{pCi} / \mathrm{L}$ & Sample \\
\hline & $\begin{array}{l}\text { Chicken Creek } \\
\text { Upstream }\end{array}$ & $3 / 1 / 2007$ & 15 & $\mathrm{~Bq} / \mathrm{L}$ & 420 & $\mathrm{pCi} / \mathrm{L}$ & Sample \\
\hline & & $3 / 30 / 2007$ & $<5.7$ & $\mathrm{~Bq} / \mathrm{L}$ & $<160$ & $\mathrm{pCi} / \mathrm{L}$ & Sample \\
\hline & FIELD BLANK & $2 / 21 / 2007$ & $<10$ & $\mathrm{~Bq} / \mathrm{L}$ & $<280$ & $\mathrm{pCi} / \mathrm{L}$ & Blank \\
\hline & & $2 / 21 / 2007$ & $<6.5$ & $\mathrm{~Bq} / \mathrm{L}$ & $<180$ & $\mathrm{pCi} / \mathrm{L}$ & Blank \\
\hline & $\begin{array}{l}\text { N. Fork Strawberry } \\
\text { Creek }\end{array}$ & $2 / 21 / 2007$ & $<6.6$ & $\mathrm{~Bq} / \mathrm{L}$ & $<180$ & pCi/L & Sample \\
\hline & & $5 / 15 / 2007$ & $<6.4$ & $\mathrm{~Bq} / \mathrm{L}$ & $<170$ & $\mathrm{pCi} / \mathrm{L}$ & Sample \\
\hline & & $8 / 21 / 2007$ & 7.7 & $\mathrm{~Bq} / \mathrm{L}$ & 210 & $\mathrm{pCi} / \mathrm{L}$ & Sample \\
\hline & & $8 / 21 / 2007$ & $<7$ & $\mathrm{~Bq} / \mathrm{L}$ & $<190$ & $\mathrm{pCi} / \mathrm{L}$ & Split \\
\hline & & $11 / 19 / 2007$ & $<6.2$ & $\mathrm{~Bq} / \mathrm{L}$ & $<170$ & $\mathrm{pCi} / \mathrm{L}$ & Sample \\
\hline & $\begin{array}{l}\text { N. Fork Strawberry } \\
\text { Creek Downstream }\end{array}$ & $3 / 1 / 2007$ & $<5.6$ & $\mathrm{~Bq} / \mathrm{L}$ & $<150$ & $\mathrm{pCi} / \mathrm{L}$ & Sample \\
\hline & $\begin{array}{l}\text { N. Fork Strawberry } \\
\text { Creek Upstream }\end{array}$ & $3 / 1 / 2007$ & $<5.6$ & $\mathrm{~Bq} / \mathrm{L}$ & $<150$ & pCi/L & Sample \\
\hline & Strawberry Creek (UC) & $2 / 21 / 2007$ & $<6.6$ & $\mathrm{~Bq} / \mathrm{L}$ & $<180$ & $\mathrm{pCi} / \mathrm{L}$ & Sample \\
\hline & & $5 / 15 / 2007$ & $<6.5$ & $\mathrm{~Bq} / \mathrm{L}$ & $<180$ & $\mathrm{pCi} / \mathrm{L}$ & Sample \\
\hline & & $8 / 21 / 2007$ & $<5.9$ & $\mathrm{~Bq} / \mathrm{L}$ & $<160$ & $\mathrm{pCi} / \mathrm{L}$ & Sample \\
\hline & & $11 / 19 / 2007$ & $<6.2$ & $\mathrm{~Bq} / \mathrm{L}$ & $<170$ & $\mathrm{pCi} / \mathrm{L}$ & Sample \\
\hline
\end{tabular}




\begin{tabular}{|c|c|c|c|c|c|}
\hline \multicolumn{2}{|c|}{ General Indicator Parameters } & \multirow[b]{2}{*}{$\begin{array}{c}\text { Collection } \\
\text { Date }\end{array}$} & \multirow[b]{2}{*}{ Result } & \multirow[b]{2}{*}{ Units } & \multirow[b]{2}{*}{$\begin{array}{c}\text { QA } \\
\text { Type }\end{array}$} \\
\hline Analyte & Location & & & & \\
\hline \multirow[t]{6}{*}{ Fecal Coliform } & NFSC-LBNL & $6 / 27 / 2007$ & 900 & MPN/100ml & Sample \\
\hline & & $7 / 5 / 2007$ & 30 & MPN/100ml & Sample \\
\hline & NFSC-LeRoy & $6 / 27 / 2007$ & 80 & MPN/100ml & Sample \\
\hline & & $7 / 5 / 2007$ & 30 & MPN/100ml & Sample \\
\hline & NFS-UCB & $6 / 27 / 2007$ & 1600 & MPN/100ml & Sample \\
\hline & & $7 / 5 / 2007$ & 3000 & MPN/100ml & Sample \\
\hline \multirow[t]{6}{*}{ Total Coliform } & NFSC-LBNL & $6 / 27 / 2007$ & 1600 & MPN/100ml & Sample \\
\hline & & $7 / 5 / 2007$ & 340 & MPN/100ml & Sample \\
\hline & NFSC-LeRoy & $6 / 27 / 2007$ & 1600 & MPN/100ml & Sample \\
\hline & & $7 / 5 / 2007$ & 1300 & MPN/100ml & Sample \\
\hline & NFS-UCB & $6 / 27 / 2007$ & 1600 & MPN/100ml & Sample \\
\hline & & $7 / 5 / 2007$ & 30000 & MPN/100ml & Sample \\
\hline
\end{tabular}




\begin{tabular}{|c|c|c|c|c|c|}
\hline \multicolumn{2}{|c|}{ Metals and Minerals } & \multirow[b]{2}{*}{$\begin{array}{l}\text { Collection } \\
\text { Date }\end{array}$} & \multirow[b]{2}{*}{ Result } & \multirow[b]{2}{*}{ Units } & \multirow[b]{2}{*}{$\begin{array}{c}\text { QA } \\
\text { Type }\end{array}$} \\
\hline Analyte & Location & & & & \\
\hline \multirow[t]{9}{*}{ Antimony } & Botanical Garden Creek & $3 / 1 / 2007$ & $<0.002$ & $\mathrm{mg} / \mathrm{L}$ & Sample \\
\hline & Cafeteria Creek & $3 / 1 / 2007$ & $<0.002$ & $\mathrm{mg} / \mathrm{L}$ & Sample \\
\hline & Chicken Creek Downstream & $3 / 1 / 2007$ & $<0.002$ & $\mathrm{mg} / \mathrm{L}$ & Sample \\
\hline & Chicken Creek Upstream & $3 / 1 / 2007$ & $<0.002$ & $\mathrm{mg} / \mathrm{L}$ & Sample \\
\hline & $\begin{array}{l}\text { N. Fork Strawberry Creek } \\
\text { Downstream }\end{array}$ & $3 / 1 / 2007$ & $<0.002$ & $\mathrm{mg} / \mathrm{L}$ & Sample \\
\hline & $\begin{array}{l}\text { N. Fork Strawberry Creek } \\
\text { Upstream }\end{array}$ & $3 / 1 / 2007$ & $<0.002$ & $\mathrm{mg} / \mathrm{L}$ & Sample \\
\hline & No Name Creek & $3 / 1 / 2007$ & $<0.002$ & $\mathrm{mg} / \mathrm{L}$ & Sample \\
\hline & Ravine Creek & $3 / 1 / 2007$ & $<0.002$ & $\mathrm{mg} / \mathrm{L}$ & Sample \\
\hline & Ten-Inch Creek & $3 / 1 / 2007$ & $<0.002$ & $\mathrm{mg} / \mathrm{L}$ & Sample \\
\hline \multirow[t]{9}{*}{ Arsenic } & Botanical Garden Creek & $3 / 1 / 2007$ & $<0.002$ & $\mathrm{mg} / \mathrm{L}$ & Sample \\
\hline & Cafeteria Creek & $3 / 1 / 2007$ & $<0.002$ & $\mathrm{mg} / \mathrm{L}$ & Sample \\
\hline & Chicken Creek Downstream & $3 / 1 / 2007$ & $<0.002$ & $\mathrm{mg} / \mathrm{L}$ & Sample \\
\hline & Chicken Creek Upstream & $3 / 1 / 2007$ & 0.0027 & $\mathrm{mg} / \mathrm{L}$ & Sample \\
\hline & $\begin{array}{l}\text { N. Fork Strawberry Creek } \\
\text { Downstream }\end{array}$ & $3 / 1 / 2007$ & $<0.002$ & $\mathrm{mg} / \mathrm{L}$ & Sample \\
\hline & $\begin{array}{l}\text { N. Fork Strawberry Creek } \\
\text { Upstream }\end{array}$ & $3 / 1 / 2007$ & $<0.002$ & $\mathrm{mg} / \mathrm{L}$ & Sample \\
\hline & No Name Creek & $3 / 1 / 2007$ & $<0.002$ & $\mathrm{mg} / \mathrm{L}$ & Sample \\
\hline & Ravine Creek & $3 / 1 / 2007$ & $<0.002$ & $\mathrm{mg} / \mathrm{L}$ & Sample \\
\hline & Ten-Inch Creek & $3 / 1 / 2007$ & $<0.002$ & $\mathrm{mg} / \mathrm{L}$ & Sample \\
\hline \multirow[t]{9}{*}{ Barium } & Botanical Garden Creek & $3 / 1 / 2007$ & 0.078 & $\mathrm{mg} / \mathrm{L}$ & Sample \\
\hline & Cafeteria Creek & $3 / 1 / 2007$ & 0.062 & $\mathrm{mg} / \mathrm{L}$ & Sample \\
\hline & Chicken Creek Downstream & $3 / 1 / 2007$ & 0.11 & $\mathrm{mg} / \mathrm{L}$ & Sample \\
\hline & Chicken Creek Upstream & $3 / 1 / 2007$ & 0.085 & $\mathrm{mg} / \mathrm{L}$ & Sample \\
\hline & $\begin{array}{l}\text { N. Fork Strawberry Creek } \\
\text { Downstream }\end{array}$ & $3 / 1 / 2007$ & 0.081 & $\mathrm{mg} / \mathrm{L}$ & Sample \\
\hline & $\begin{array}{l}\text { N. Fork Strawberry Creek } \\
\text { Upstream }\end{array}$ & $3 / 1 / 2007$ & 0.083 & $\mathrm{mg} / \mathrm{L}$ & Sample \\
\hline & No Name Creek & $3 / 1 / 2007$ & 0.11 & $\mathrm{mg} / \mathrm{L}$ & Sample \\
\hline & Ravine Creek & $3 / 1 / 2007$ & 0.073 & $\mathrm{mg} / \mathrm{L}$ & Sample \\
\hline & Ten-Inch Creek & $3 / 1 / 2007$ & 0.057 & $\mathrm{mg} / \mathrm{L}$ & Sample \\
\hline \multirow[t]{4}{*}{ Beryllium } & Botanical Garden Creek & $3 / 1 / 2007$ & $<0.001$ & $\mathrm{mg} / \mathrm{L}$ & Sample \\
\hline & Cafeteria Creek & $3 / 1 / 2007$ & $<0.001$ & $\mathrm{mg} / \mathrm{L}$ & Sample \\
\hline & Chicken Creek Downstream & $3 / 1 / 2007$ & $<0.001$ & $\mathrm{mg} / \mathrm{L}$ & Sample \\
\hline & Chicken Creek Upstream & $3 / 1 / 2007$ & $<0.001$ & $\mathrm{mg} / \mathrm{L}$ & Sample \\
\hline
\end{tabular}




\begin{tabular}{|c|c|c|c|c|c|}
\hline \multicolumn{2}{|c|}{ Metals and Minerals } & \multirow[b]{2}{*}{$\begin{array}{l}\text { Collection } \\
\text { Date }\end{array}$} & \multirow[b]{2}{*}{ Result } & \multirow[b]{2}{*}{ Units } & \multirow[b]{2}{*}{$\begin{array}{l}\text { QA } \\
\text { Type }\end{array}$} \\
\hline Analyte & Location & & & & \\
\hline \multirow[t]{5}{*}{ Beryllium } & $\begin{array}{l}\text { N. Fork Strawberry Creek } \\
\text { Downstream }\end{array}$ & $3 / 1 / 2007$ & $<0.001$ & $\mathrm{mg} / \mathrm{L}$ & Sample \\
\hline & $\begin{array}{l}\text { N. Fork Strawberry Creek } \\
\text { Upstream }\end{array}$ & $3 / 1 / 2007$ & $<0.001$ & $\mathrm{mg} / \mathrm{L}$ & Sample \\
\hline & No Name Creek & $3 / 1 / 2007$ & $<0.001$ & $\mathrm{mg} / \mathrm{L}$ & Sample \\
\hline & Ravine Creek & $3 / 1 / 2007$ & $<0.001$ & $\mathrm{mg} / \mathrm{L}$ & Sample \\
\hline & Ten-Inch Creek & $3 / 1 / 2007$ & $<0.001$ & $\mathrm{mg} / \mathrm{L}$ & Sample \\
\hline \multirow[t]{9}{*}{ Cadmium } & Botanical Garden Creek & $3 / 1 / 2007$ & $<0.001$ & $\mathrm{mg} / \mathrm{L}$ & Sample \\
\hline & Cafeteria Creek & $3 / 1 / 2007$ & $<0.001$ & $\mathrm{mg} / \mathrm{L}$ & Sample \\
\hline & Chicken Creek Downstream & $3 / 1 / 2007$ & $<0.001$ & $\mathrm{mg} / \mathrm{L}$ & Sample \\
\hline & Chicken Creek Upstream & $3 / 1 / 2007$ & $<0.001$ & $\mathrm{mg} / \mathrm{L}$ & Sample \\
\hline & $\begin{array}{l}\text { N. Fork Strawberry Creek } \\
\text { Downstream }\end{array}$ & $3 / 1 / 2007$ & $<0.001$ & $\mathrm{mg} / \mathrm{L}$ & Sample \\
\hline & $\begin{array}{l}\text { N. Fork Strawberry Creek } \\
\text { Upstream }\end{array}$ & $3 / 1 / 2007$ & $<0.001$ & $\mathrm{mg} / \mathrm{L}$ & Sample \\
\hline & No Name Creek & $3 / 1 / 2007$ & $<0.001$ & $\mathrm{mg} / \mathrm{L}$ & Sample \\
\hline & Ravine Creek & $3 / 1 / 2007$ & $<0.001$ & $\mathrm{mg} / \mathrm{L}$ & Sample \\
\hline & Ten-Inch Creek & $3 / 1 / 2007$ & $<0.001$ & $\mathrm{mg} / \mathrm{L}$ & Sample \\
\hline \multirow[t]{9}{*}{ Chromium } & Botanical Garden Creek & $3 / 1 / 2007$ & $<0.01$ & $\mathrm{mg} / \mathrm{L}$ & Sample \\
\hline & Cafeteria Creek & $3 / 1 / 2007$ & $<0.01$ & $\mathrm{mg} / \mathrm{L}$ & Sample \\
\hline & Chicken Creek Downstream & $3 / 1 / 2007$ & $<0.01$ & $\mathrm{mg} / \mathrm{L}$ & Sample \\
\hline & Chicken Creek Upstream & $3 / 1 / 2007$ & $<0.01$ & $\mathrm{mg} / \mathrm{L}$ & Sample \\
\hline & $\begin{array}{l}\text { N. Fork Strawberry Creek } \\
\text { Downstream }\end{array}$ & $3 / 1 / 2007$ & $<0.01$ & $\mathrm{mg} / \mathrm{L}$ & Sample \\
\hline & $\begin{array}{l}\text { N. Fork Strawberry Creek } \\
\text { Upstream }\end{array}$ & $3 / 1 / 2007$ & $<0.01$ & $\mathrm{mg} / \mathrm{L}$ & Sample \\
\hline & No Name Creek & $3 / 1 / 2007$ & $<0.01$ & $\mathrm{mg} / \mathrm{L}$ & Sample \\
\hline & Ravine Creek & $3 / 1 / 2007$ & $<0.01$ & $\mathrm{mg} / \mathrm{L}$ & Sample \\
\hline & Ten-Inch Creek & $3 / 1 / 2007$ & $<0.01$ & $\mathrm{mg} / \mathrm{L}$ & Sample \\
\hline \multirow[t]{8}{*}{ Cobalt } & Botanical Garden Creek & $3 / 1 / 2007$ & $<0.05$ & $\mathrm{mg} / \mathrm{L}$ & Sample \\
\hline & Cafeteria Creek & $3 / 1 / 2007$ & $<0.05$ & $\mathrm{mg} / \mathrm{L}$ & Sample \\
\hline & Chicken Creek Downstream & $3 / 1 / 2007$ & $<0.05$ & $\mathrm{mg} / \mathrm{L}$ & Sample \\
\hline & Chicken Creek Upstream & $3 / 1 / 2007$ & $<0.05$ & $\mathrm{mg} / \mathrm{L}$ & Sample \\
\hline & $\begin{array}{l}\text { N. Fork Strawberry Creek } \\
\text { Downstream }\end{array}$ & $3 / 1 / 2007$ & $<0.05$ & $\mathrm{mg} / \mathrm{L}$ & Sample \\
\hline & $\begin{array}{l}\text { N. Fork Strawberry Creek } \\
\text { Upstream }\end{array}$ & $3 / 1 / 2007$ & $<0.05$ & $\mathrm{mg} / \mathrm{L}$ & Sample \\
\hline & No Name Creek & $3 / 1 / 2007$ & $<0.05$ & $\mathrm{mg} / \mathrm{L}$ & Sample \\
\hline & Ravine Creek & $3 / 1 / 2007$ & $<0.05$ & $\mathrm{mg} / \mathrm{L}$ & Sample \\
\hline
\end{tabular}




\begin{tabular}{|c|c|c|c|c|c|}
\hline \multicolumn{2}{|c|}{ Metals and Minerals } & \multirow[b]{2}{*}{$\begin{array}{c}\text { Collection } \\
\text { Date }\end{array}$} & \multirow[b]{2}{*}{ Result } & \multirow[b]{2}{*}{ Units } & \multirow[b]{2}{*}{$\begin{array}{c}\text { QA } \\
\text { Type }\end{array}$} \\
\hline Analyte & Location & & & & \\
\hline Cobalt & Ten-Inch Creek & $3 / 1 / 2007$ & $<0.05$ & $\mathrm{mg} / \mathrm{L}$ & Sample \\
\hline \multirow[t]{9}{*}{ Copper } & Botanical Garden Creek & $3 / 1 / 2007$ & $<0.01$ & $\mathrm{mg} / \mathrm{L}$ & Sample \\
\hline & Cafeteria Creek & $3 / 1 / 2007$ & $<0.01$ & $\mathrm{mg} / \mathrm{L}$ & Sample \\
\hline & Chicken Creek Downstream & $3 / 1 / 2007$ & $<0.01$ & $\mathrm{mg} / \mathrm{L}$ & Sample \\
\hline & Chicken Creek Upstream & $3 / 1 / 2007$ & $<0.01$ & $\mathrm{mg} / \mathrm{L}$ & Sample \\
\hline & $\begin{array}{l}\text { N. Fork Strawberry Creek } \\
\text { Downstream }\end{array}$ & $3 / 1 / 2007$ & $<0.01$ & $\mathrm{mg} / \mathrm{L}$ & Sample \\
\hline & $\begin{array}{l}\text { N. Fork Strawberry Creek } \\
\text { Upstream }\end{array}$ & $3 / 1 / 2007$ & $<0.01$ & $\mathrm{mg} / \mathrm{L}$ & Sample \\
\hline & No Name Creek & $3 / 1 / 2007$ & $<0.01$ & $\mathrm{mg} / \mathrm{L}$ & Sample \\
\hline & Ravine Creek & $3 / 1 / 2007$ & $<0.01$ & $\mathrm{mg} / \mathrm{L}$ & Sample \\
\hline & Ten-Inch Creek & $3 / 1 / 2007$ & $<0.01$ & $\mathrm{mg} / \mathrm{L}$ & Sample \\
\hline \multirow[t]{9}{*}{ Lead } & Botanical Garden Creek & $3 / 1 / 2007$ & $<0.001$ & $\mathrm{mg} / \mathrm{L}$ & Sample \\
\hline & Cafeteria Creek & $3 / 1 / 2007$ & $<0.001$ & $\mathrm{mg} / \mathrm{L}$ & Sample \\
\hline & Chicken Creek Downstream & $3 / 1 / 2007$ & $<0.001$ & $\mathrm{mg} / \mathrm{L}$ & Sample \\
\hline & Chicken Creek Upstream & $3 / 1 / 2007$ & $<0.001$ & $\mathrm{mg} / \mathrm{L}$ & Sample \\
\hline & $\begin{array}{l}\text { N. Fork Strawberry Creek } \\
\text { Downstream }\end{array}$ & $3 / 1 / 2007$ & $<0.001$ & $\mathrm{mg} / \mathrm{L}$ & Sample \\
\hline & $\begin{array}{l}\text { N. Fork Strawberry Creek } \\
\text { Upstream }\end{array}$ & $3 / 1 / 2007$ & $<0.001$ & $\mathrm{mg} / \mathrm{L}$ & Sample \\
\hline & No Name Creek & $3 / 1 / 2007$ & $<0.001$ & $\mathrm{mg} / \mathrm{L}$ & Sample \\
\hline & Ravine Creek & $3 / 1 / 2007$ & $<0.001$ & $\mathrm{mg} / \mathrm{L}$ & Sample \\
\hline & Ten-Inch Creek & $3 / 1 / 2007$ & $<0.001$ & $\mathrm{mg} / \mathrm{L}$ & Sample \\
\hline \multirow[t]{14}{*}{ Mercury } & Botanical Garden Creek & $3 / 1 / 2007$ & $<0.0002$ & $\mathrm{mg} / \mathrm{L}$ & Sample \\
\hline & Cafeteria Creek & $3 / 1 / 2007$ & $<0.0002$ & $\mathrm{mg} / \mathrm{L}$ & Sample \\
\hline & Chicken Creek & $2 / 21 / 2007$ & $<0.0002$ & $\mathrm{mg} / \mathrm{L}$ & Sample \\
\hline & & $2 / 21 / 2007$ & $<0.0002$ & $\mathrm{mg} / \mathrm{L}$ & Split \\
\hline & & $5 / 15 / 2007$ & $<0.0002$ & $\mathrm{mg} / \mathrm{L}$ & Sample \\
\hline & & $8 / 21 / 2007$ & $<0.0002$ & $\mathrm{mg} / \mathrm{L}$ & Sample \\
\hline & & $11 / 19 / 2007$ & $<0.0002$ & $\mathrm{mg} / \mathrm{L}$ & Sample \\
\hline & Chicken Creek Downstream & $3 / 1 / 2007$ & $<0.0002$ & $\mathrm{mg} / \mathrm{L}$ & Sample \\
\hline & Chicken Creek Upstream & $3 / 1 / 2007$ & $<0.0002$ & $\mathrm{mg} / \mathrm{L}$ & Sample \\
\hline & FIELD BLANK & $2 / 21 / 2007$ & $<0.0002$ & $\mathrm{mg} / \mathrm{L}$ & Blank \\
\hline & & $2 / 21 / 2007$ & $<0.0002$ & $\mathrm{mg} / \mathrm{L}$ & Blank \\
\hline & N. Fork Strawberry Creek & 2/21/2007 & $<0.0002$ & $\mathrm{mg} / \mathrm{L}$ & Sample \\
\hline & & $5 / 15 / 2007$ & $<0.0002$ & $\mathrm{mg} / \mathrm{L}$ & Sample \\
\hline & & $8 / 21 / 2007$ & 0.00041 & $\mathrm{mg} / \mathrm{L}$ & Sample \\
\hline
\end{tabular}




\begin{tabular}{|c|c|c|c|c|c|}
\hline \multicolumn{2}{|c|}{ Metals and Minerals } & \multirow[b]{2}{*}{$\begin{array}{l}\text { Collection } \\
\text { Date }\end{array}$} & \multirow[b]{2}{*}{ Result } & \multirow[b]{2}{*}{ Units } & \multirow[b]{2}{*}{$\begin{array}{c}\text { QA } \\
\text { Type }\end{array}$} \\
\hline Analyte & Location & & & & \\
\hline \multirow[t]{9}{*}{ Mercury } & N. Fork Strawberry Creek & $8 / 21 / 2007$ & $<0.0002$ & $\mathrm{mg} / \mathrm{L}$ & Split \\
\hline & & $11 / 19 / 2007$ & $<0.0002$ & $\mathrm{mg} / \mathrm{L}$ & Sample \\
\hline & $\begin{array}{l}\text { N. Fork Strawberry Creek } \\
\text { Downstream }\end{array}$ & $3 / 1 / 2007$ & $<0.0002$ & $\mathrm{mg} / \mathrm{L}$ & Sample \\
\hline & $\begin{array}{l}\text { N. Fork Strawberry Creek } \\
\text { Upstream }\end{array}$ & $3 / 1 / 2007$ & $<0.0002$ & $\mathrm{mg} / \mathrm{L}$ & Sample \\
\hline & No Name Creek & $3 / 1 / 2007$ & $<0.0002$ & $\mathrm{mg} / \mathrm{L}$ & Sample \\
\hline & Strawberry Creek (UC) & $2 / 21 / 2007$ & $<0.0002$ & $\mathrm{mg} / \mathrm{L}$ & Sample \\
\hline & & $5 / 15 / 2007$ & $<0.0002$ & $\mathrm{mg} / \mathrm{L}$ & Sample \\
\hline & & $8 / 21 / 2007$ & $<0.0002$ & $\mathrm{mg} / \mathrm{L}$ & Sample \\
\hline & & $11 / 19 / 2007$ & $<0.0002$ & $\mathrm{mg} / \mathrm{L}$ & Sample \\
\hline \multirow[t]{9}{*}{ Molybdenum } & Botanical Garden Creek & $3 / 1 / 2007$ & $<0.05$ & $\mathrm{mg} / \mathrm{L}$ & Sample \\
\hline & Cafeteria Creek & $3 / 1 / 2007$ & $<0.05$ & $\mathrm{mg} / \mathrm{L}$ & Sample \\
\hline & Chicken Creek Downstream & $3 / 1 / 2007$ & $<0.05$ & $\mathrm{mg} / \mathrm{L}$ & Sample \\
\hline & Chicken Creek Upstream & $3 / 1 / 2007$ & $<0.05$ & $\mathrm{mg} / \mathrm{L}$ & Sample \\
\hline & $\begin{array}{l}\text { N. Fork Strawberry Creek } \\
\text { Downstream }\end{array}$ & $3 / 1 / 2007$ & $<0.05$ & $\mathrm{mg} / \mathrm{L}$ & Sample \\
\hline & $\begin{array}{l}\text { N. Fork Strawberry Creek } \\
\text { Upstream }\end{array}$ & $3 / 1 / 2007$ & $<0.05$ & $\mathrm{mg} / \mathrm{L}$ & Sample \\
\hline & No Name Creek & $3 / 1 / 2007$ & $<0.05$ & $\mathrm{mg} / \mathrm{L}$ & Sample \\
\hline & Ravine Creek & $3 / 1 / 2007$ & $<0.05$ & $\mathrm{mg} / \mathrm{L}$ & Sample \\
\hline & Ten-Inch Creek & $3 / 1 / 2007$ & $<0.05$ & $\mathrm{mg} / \mathrm{L}$ & Sample \\
\hline \multirow[t]{9}{*}{ Nickel } & Botanical Garden Creek & $3 / 1 / 2007$ & $<0.01$ & $\mathrm{mg} / \mathrm{L}$ & Sample \\
\hline & Cafeteria Creek & $3 / 1 / 2007$ & $<0.01$ & $\mathrm{mg} / \mathrm{L}$ & Sample \\
\hline & Chicken Creek Downstream & $3 / 1 / 2007$ & $<0.01$ & $\mathrm{mg} / \mathrm{L}$ & Sample \\
\hline & Chicken Creek Upstream & $3 / 1 / 2007$ & $<0.01$ & $\mathrm{mg} / \mathrm{L}$ & Sample \\
\hline & $\begin{array}{l}\text { N. Fork Strawberry Creek } \\
\text { Downstream }\end{array}$ & $3 / 1 / 2007$ & $<0.01$ & $\mathrm{mg} / \mathrm{L}$ & Sample \\
\hline & $\begin{array}{l}\text { N. Fork Strawberry Creek } \\
\text { Upstream }\end{array}$ & $3 / 1 / 2007$ & $<0.01$ & $\mathrm{mg} / \mathrm{L}$ & Sample \\
\hline & No Name Creek & $3 / 1 / 2007$ & $<0.01$ & $\mathrm{mg} / \mathrm{L}$ & Sample \\
\hline & Ravine Creek & $3 / 1 / 2007$ & $<0.01$ & $\mathrm{mg} / \mathrm{L}$ & Sample \\
\hline & Ten-Inch Creek & $3 / 1 / 2007$ & $<0.01$ & $\mathrm{mg} / \mathrm{L}$ & Sample \\
\hline \multirow[t]{4}{*}{ Selenium } & Botanical Garden Creek & $3 / 1 / 2007$ & 0.0023 & $\mathrm{mg} / \mathrm{L}$ & Sample \\
\hline & Cafeteria Creek & $3 / 1 / 2007$ & $<0.002$ & $\mathrm{mg} / \mathrm{L}$ & Sample \\
\hline & Chicken Creek Downstream & $3 / 1 / 2007$ & $<0.002$ & $\mathrm{mg} / \mathrm{L}$ & Sample \\
\hline & Chicken Creek Upstream & $3 / 1 / 2007$ & 0.0026 & $\mathrm{mg} / \mathrm{L}$ & Sample \\
\hline
\end{tabular}




\begin{tabular}{|c|c|c|c|c|c|}
\hline \multicolumn{2}{|c|}{ Metals and Minerals } & \multirow[b]{2}{*}{$\begin{array}{l}\text { Collection } \\
\text { Date }\end{array}$} & \multirow[b]{2}{*}{ Result } & \multirow[b]{2}{*}{ Units } & \multirow[b]{2}{*}{$\begin{array}{l}\text { QA } \\
\text { Type }\end{array}$} \\
\hline Analyte & Location & & & & \\
\hline \multirow[t]{5}{*}{ Selenium } & $\begin{array}{l}\text { N. Fork Strawberry Creek } \\
\text { Downstream }\end{array}$ & $3 / 1 / 2007$ & $<0.002$ & $\mathrm{mg} / \mathrm{L}$ & Sample \\
\hline & $\begin{array}{l}\text { N. Fork Strawberry Creek } \\
\text { Upstream }\end{array}$ & $3 / 1 / 2007$ & $<0.002$ & $\mathrm{mg} / \mathrm{L}$ & Sample \\
\hline & No Name Creek & $3 / 1 / 2007$ & $<0.002$ & $\mathrm{mg} / \mathrm{L}$ & Sample \\
\hline & Ravine Creek & $3 / 1 / 2007$ & $<0.002$ & $\mathrm{mg} / \mathrm{L}$ & Sample \\
\hline & Ten-Inch Creek & $3 / 1 / 2007$ & $<0.002$ & $\mathrm{mg} / \mathrm{L}$ & Sample \\
\hline \multirow[t]{9}{*}{ Silver } & Botanical Garden Creek & $3 / 1 / 2007$ & $<0.01$ & $\mathrm{mg} / \mathrm{L}$ & Sample \\
\hline & Cafeteria Creek & $3 / 1 / 2007$ & $<0.01$ & $\mathrm{mg} / \mathrm{L}$ & Sample \\
\hline & Chicken Creek Downstream & $3 / 1 / 2007$ & $<0.01$ & $\mathrm{mg} / \mathrm{L}$ & Sample \\
\hline & Chicken Creek Upstream & $3 / 1 / 2007$ & $<0.01$ & $\mathrm{mg} / \mathrm{L}$ & Sample \\
\hline & $\begin{array}{l}\text { N. Fork Strawberry Creek } \\
\text { Downstream }\end{array}$ & 3/1/2007 & $<0.01$ & $\mathrm{mg} / \mathrm{L}$ & Sample \\
\hline & $\begin{array}{l}\text { N. Fork Strawberry Creek } \\
\text { Upstream }\end{array}$ & $3 / 1 / 2007$ & $<0.01$ & $\mathrm{mg} / \mathrm{L}$ & Sample \\
\hline & No Name Creek & $3 / 1 / 2007$ & $<0.01$ & $\mathrm{mg} / \mathrm{L}$ & Sample \\
\hline & Ravine Creek & $3 / 1 / 2007$ & $<0.01$ & $\mathrm{mg} / \mathrm{L}$ & Sample \\
\hline & Ten-Inch Creek & $3 / 1 / 2007$ & $<0.01$ & $\mathrm{mg} / \mathrm{L}$ & Sample \\
\hline \multirow[t]{9}{*}{ Thallium } & Botanical Garden Creek & $3 / 1 / 2007$ & $<0.001$ & $\mathrm{mg} / \mathrm{L}$ & Sample \\
\hline & Cafeteria Creek & $3 / 1 / 2007$ & $<0.001$ & $\mathrm{mg} / \mathrm{L}$ & Sample \\
\hline & Chicken Creek Downstream & $3 / 1 / 2007$ & $<0.001$ & $\mathrm{mg} / \mathrm{L}$ & Sample \\
\hline & Chicken Creek Upstream & $3 / 1 / 2007$ & $<0.001$ & $\mathrm{mg} / \mathrm{L}$ & Sample \\
\hline & $\begin{array}{l}\text { N. Fork Strawberry Creek } \\
\text { Downstream }\end{array}$ & $3 / 1 / 2007$ & $<0.001$ & $\mathrm{mg} / \mathrm{L}$ & Sample \\
\hline & $\begin{array}{l}\text { N. Fork Strawberry Creek } \\
\text { Upstream }\end{array}$ & $3 / 1 / 2007$ & $<0.001$ & $\mathrm{mg} / \mathrm{L}$ & Sample \\
\hline & No Name Creek & $3 / 1 / 2007$ & $<0.001$ & $\mathrm{mg} / \mathrm{L}$ & Sample \\
\hline & Ravine Creek & $3 / 1 / 2007$ & $<0.001$ & $\mathrm{mg} / \mathrm{L}$ & Sample \\
\hline & Ten-Inch Creek & $3 / 1 / 2007$ & $<0.001$ & $\mathrm{mg} / \mathrm{L}$ & Sample \\
\hline \multirow[t]{8}{*}{ Vanadium } & Botanical Garden Creek & $3 / 1 / 2007$ & $<0.01$ & $\mathrm{mg} / \mathrm{L}$ & Sample \\
\hline & Cafeteria Creek & $3 / 1 / 2007$ & $<0.01$ & $\mathrm{mg} / \mathrm{L}$ & Sample \\
\hline & Chicken Creek Downstream & $3 / 1 / 2007$ & $<0.01$ & $\mathrm{mg} / \mathrm{L}$ & Sample \\
\hline & Chicken Creek Upstream & $3 / 1 / 2007$ & 0.016 & $\mathrm{mg} / \mathrm{L}$ & Sample \\
\hline & $\begin{array}{l}\text { N. Fork Strawberry Creek } \\
\text { Downstream }\end{array}$ & $3 / 1 / 2007$ & $<0.01$ & $\mathrm{mg} / \mathrm{L}$ & Sample \\
\hline & $\begin{array}{l}\text { N. Fork Strawberry Creek } \\
\text { Upstream }\end{array}$ & $3 / 1 / 2007$ & $<0.01$ & $\mathrm{mg} / \mathrm{L}$ & Sample \\
\hline & No Name Creek & $3 / 1 / 2007$ & $<0.01$ & $\mathrm{mg} / \mathrm{L}$ & Sample \\
\hline & Ravine Creek & $3 / 1 / 2007$ & $<0.01$ & $\mathrm{mg} / \mathrm{L}$ & Sample \\
\hline
\end{tabular}




\begin{tabular}{|l|l|c|c|c|c|}
\hline \multicolumn{2}{|c|}{ Metals and Minerals } & \multicolumn{1}{c|}{} & \\
\hline Analyte & \multicolumn{1}{|c|}{ Location } & $\begin{array}{c}\text { Collection } \\
\text { Date }\end{array}$ & Result & Units & $\begin{array}{c}\text { QA } \\
\text { Type }\end{array}$ \\
\hline Vanadium & Ten-Inch Creek & $3 / 1 / 2007$ & $<0.01$ & $\mathrm{mg} / \mathrm{L}$ & Sample \\
\hline Zinc & Botanical Garden Creek & $3 / 1 / 2007$ & $<0.01$ & $\mathrm{mg} / \mathrm{L}$ & Sample \\
\hline & Cafeteria Creek & $3 / 1 / 2007$ & 0.025 & $\mathrm{mg} / \mathrm{L}$ & Sample \\
\hline & Chicken Creek Downstream & $3 / 1 / 2007$ & 0.011 & $\mathrm{mg} / \mathrm{L}$ & Sample \\
\hline & Chicken Creek Upstream & $3 / 1 / 2007$ & 0.041 & $\mathrm{mg} / \mathrm{L}$ & Sample \\
\hline & N. Fork Strawberry Creek & $3 / 1 / 2007$ & 0.023 & $\mathrm{mg} / \mathrm{L}$ & Sample \\
\hline & Downstream & $3 / 1 / 2007$ & 0.025 & $\mathrm{mg} / \mathrm{L}$ & Sample \\
\hline & $\begin{array}{l}\text { N. Fork Strawberry Creek } \\
\text { Upstream }\end{array}$ & $3 / 1 / 2007$ & $<0.01$ & $\mathrm{mg} / \mathrm{L}$ & Sample \\
\hline & No Name Creek & $3 / 1 / 2007$ & $<0.01$ & $\mathrm{mg} / \mathrm{L}$ & Sample \\
\hline & Ravine Creek & $3 / 1 / 2007$ & 0.011 & $\mathrm{mg} / \mathrm{L}$ & Sample \\
\hline & Ten-Inch Creek & & & & \\
\hline
\end{tabular}




\begin{tabular}{|c|c|c|c|c|c|}
\hline \multicolumn{2}{|c|}{ Volatile Organic Compounds } & \multirow[b]{2}{*}{$\begin{array}{c}\text { Collection } \\
\text { Date }\end{array}$} & \multirow[b]{2}{*}{ Result } & \multirow[b]{2}{*}{ Units } & \multirow[b]{2}{*}{$\begin{array}{c}\text { QA } \\
\text { Type }\end{array}$} \\
\hline Analyte & Location & & & & \\
\hline \multirow[t]{9}{*}{ 1,1,1,2-Tetrachloroethane } & Botanical Garden Creek & $3 / 1 / 2007$ & $<2$ & $\mu g / L$ & Sample \\
\hline & Cafeteria Creek & $3 / 1 / 2007$ & $<2$ & $\mu g / L$ & Sample \\
\hline & Chicken Creek Downstream & $3 / 1 / 2007$ & $<2$ & $\mu g / L$ & Sample \\
\hline & Chicken Creek Upstream & $3 / 1 / 2007$ & $<2$ & $\mu g / L$ & Sample \\
\hline & $\begin{array}{l}\text { N. Fork Strawberry Creek } \\
\text { Downstream }\end{array}$ & $3 / 1 / 2007$ & $<2$ & $\mu \mathrm{g} / \mathrm{L}$ & Sample \\
\hline & $\begin{array}{l}\text { N. Fork Strawberry Creek } \\
\text { Upstream }\end{array}$ & $3 / 1 / 2007$ & $<2$ & $\mu g / L$ & Sample \\
\hline & No Name Creek & $3 / 1 / 2007$ & $<2$ & $\mu g / L$ & Sample \\
\hline & Ravine Creek & $3 / 1 / 2007$ & $<2$ & $\mu \mathrm{g} / \mathrm{L}$ & Sample \\
\hline & Ten-Inch Creek & $3 / 1 / 2007$ & $<2$ & $\mu g / L$ & Sample \\
\hline \multirow[t]{9}{*}{ 1,1,1-Trichloroethane } & Botanical Garden Creek & $3 / 1 / 2007$ & $<1$ & $\mu g / L$ & Sample \\
\hline & Cafeteria Creek & $3 / 1 / 2007$ & $<1$ & $\mu g / L$ & Sample \\
\hline & Chicken Creek Downstream & $3 / 1 / 2007$ & $<1$ & $\mu g / L$ & Sample \\
\hline & Chicken Creek Upstream & $3 / 1 / 2007$ & $<1$ & $\mu g / L$ & Sample \\
\hline & $\begin{array}{l}\text { N. Fork Strawberry Creek } \\
\text { Downstream }\end{array}$ & $3 / 1 / 2007$ & $<1$ & $\mu \mathrm{g} / \mathrm{L}$ & Sample \\
\hline & $\begin{array}{l}\text { N. Fork Strawberry Creek } \\
\text { Upstream }\end{array}$ & $3 / 1 / 2007$ & $<1$ & $\mu g / L$ & Sample \\
\hline & No Name Creek & $3 / 1 / 2007$ & $<1$ & $\mu \mathrm{g} / \mathrm{L}$ & Sample \\
\hline & Ravine Creek & $3 / 1 / 2007$ & $<1$ & $\mu \mathrm{g} / \mathrm{L}$ & Sample \\
\hline & Ten-Inch Creek & $3 / 1 / 2007$ & $<1$ & $\mu g / L$ & Sample \\
\hline \multirow[t]{9}{*}{ 1,1,2,2-Tetrachloroethane } & Botanical Garden Creek & $3 / 1 / 2007$ & $<1$ & $\mu g / L$ & Sample \\
\hline & Cafeteria Creek & $3 / 1 / 2007$ & $<1$ & $\mu g / L$ & Sample \\
\hline & Chicken Creek Downstream & $3 / 1 / 2007$ & $<1$ & $\mu g / L$ & Sample \\
\hline & Chicken Creek Upstream & $3 / 1 / 2007$ & $<1$ & $\mu g / L$ & Sample \\
\hline & $\begin{array}{l}\text { N. Fork Strawberry Creek } \\
\text { Downstream }\end{array}$ & $3 / 1 / 2007$ & $<1$ & $\mu g / L$ & Sample \\
\hline & $\begin{array}{l}\text { N. Fork Strawberry Creek } \\
\text { Upstream }\end{array}$ & $3 / 1 / 2007$ & $<1$ & $\mu \mathrm{g} / \mathrm{L}$ & Sample \\
\hline & No Name Creek & $3 / 1 / 2007$ & $<1$ & $\mu \mathrm{g} / \mathrm{L}$ & Sample \\
\hline & Ravine Creek & $3 / 1 / 2007$ & $<1$ & $\mu g / L$ & Sample \\
\hline & Ten-Inch Creek & $3 / 1 / 2007$ & $<1$ & $\mu g / L$ & Sample \\
\hline \multirow[t]{4}{*}{ 1,1,2-Trichloroethane } & Botanical Garden Creek & $3 / 1 / 2007$ & $<1$ & $\mu g / L$ & Sample \\
\hline & Cafeteria Creek & $3 / 1 / 2007$ & $<1$ & $\mu \mathrm{g} / \mathrm{L}$ & Sample \\
\hline & Chicken Creek Downstream & $3 / 1 / 2007$ & $<1$ & $\mu \mathrm{g} / \mathrm{L}$ & Sample \\
\hline & Chicken Creek Upstream & $3 / 1 / 2007$ & $<1$ & $\mu g / L$ & Sample \\
\hline
\end{tabular}




\begin{tabular}{|c|c|c|c|c|c|}
\hline \multicolumn{2}{|c|}{ Volatile Organic Compounds } & \multirow[b]{2}{*}{$\begin{array}{c}\text { Collection } \\
\text { Date }\end{array}$} & \multirow[b]{2}{*}{ Result } & \multirow[b]{2}{*}{ Units } & \multirow[b]{2}{*}{$\begin{array}{c}\text { QA } \\
\text { Type }\end{array}$} \\
\hline Analyte & Location & & & & \\
\hline \multirow[t]{5}{*}{ 1,1,2-Trichloroethane } & $\begin{array}{l}\text { N. Fork Strawberry Creek } \\
\text { Downstream }\end{array}$ & $3 / 1 / 2007$ & $<1$ & $\mu \mathrm{g} / \mathrm{L}$ & Sample \\
\hline & $\begin{array}{l}\text { N. Fork Strawberry Creek } \\
\text { Upstream }\end{array}$ & 3/1/2007 & $<1$ & $\mu \mathrm{g} / \mathrm{L}$ & Sample \\
\hline & No Name Creek & 3/1/2007 & $<1$ & $\mu \mathrm{g} / \mathrm{L}$ & Sample \\
\hline & Ravine Creek & $3 / 1 / 2007$ & $<1$ & $\mu \mathrm{g} / \mathrm{L}$ & Sample \\
\hline & Ten-Inch Creek & $3 / 1 / 2007$ & $<1$ & $\mu \mathrm{g} / \mathrm{L}$ & Sample \\
\hline \multirow[t]{9}{*}{ 1,1-Dichloroethane } & Botanical Garden Creek & $3 / 1 / 2007$ & $<1$ & $\mu \mathrm{g} / \mathrm{L}$ & Sample \\
\hline & Cafeteria Creek & $3 / 1 / 2007$ & $<1$ & $\mu \mathrm{g} / \mathrm{L}$ & Sample \\
\hline & Chicken Creek Downstream & $3 / 1 / 2007$ & $<1$ & $\mu \mathrm{g} / \mathrm{L}$ & Sample \\
\hline & Chicken Creek Upstream & $3 / 1 / 2007$ & $<1$ & $\mu \mathrm{g} / \mathrm{L}$ & Sample \\
\hline & $\begin{array}{l}\text { N. Fork Strawberry Creek } \\
\text { Downstream }\end{array}$ & $3 / 1 / 2007$ & $<1$ & $\mu \mathrm{g} / \mathrm{L}$ & Sample \\
\hline & $\begin{array}{l}\text { N. Fork Strawberry Creek } \\
\text { Upstream }\end{array}$ & $3 / 1 / 2007$ & $<1$ & $\mu \mathrm{g} / \mathrm{L}$ & Sample \\
\hline & No Name Creek & $3 / 1 / 2007$ & $<1$ & $\mu \mathrm{g} / \mathrm{L}$ & Sample \\
\hline & Ravine Creek & $3 / 1 / 2007$ & $<1$ & $\mu \mathrm{g} / \mathrm{L}$ & Sample \\
\hline & Ten-Inch Creek & $3 / 1 / 2007$ & $<1$ & $\mu \mathrm{g} / \mathrm{L}$ & Sample \\
\hline \multirow[t]{9}{*}{ 1,1-Dichloroethene } & Botanical Garden Creek & $3 / 1 / 2007$ & $<1$ & $\mu \mathrm{g} / \mathrm{L}$ & Sample \\
\hline & Cafeteria Creek & 3/1/2007 & $<1$ & $\mu \mathrm{g} / \mathrm{L}$ & Sample \\
\hline & Chicken Creek Downstream & $3 / 1 / 2007$ & $<1$ & $\mu \mathrm{g} / \mathrm{L}$ & Sample \\
\hline & Chicken Creek Upstream & $3 / 1 / 2007$ & $<1$ & $\mu \mathrm{g} / \mathrm{L}$ & Sample \\
\hline & $\begin{array}{l}\text { N. Fork Strawberry Creek } \\
\text { Downstream }\end{array}$ & $3 / 1 / 2007$ & $<1$ & $\mu \mathrm{g} / \mathrm{L}$ & Sample \\
\hline & $\begin{array}{l}\text { N. Fork Strawberry Creek } \\
\text { Upstream }\end{array}$ & $3 / 1 / 2007$ & $<1$ & $\mu g / L$ & Sample \\
\hline & No Name Creek & $3 / 1 / 2007$ & $<1$ & $\mu \mathrm{g} / \mathrm{L}$ & Sample \\
\hline & Ravine Creek & 3/1/2007 & $<1$ & $\mu \mathrm{g} / \mathrm{L}$ & Sample \\
\hline & Ten-Inch Creek & $3 / 1 / 2007$ & $<1$ & $\mu \mathrm{g} / \mathrm{L}$ & Sample \\
\hline \multirow[t]{8}{*}{ 1,1-Dichloropropene } & Botanical Garden Creek & $3 / 1 / 2007$ & $<1$ & $\mu \mathrm{g} / \mathrm{L}$ & Sample \\
\hline & Cafeteria Creek & 3/1/2007 & $<1$ & $\mu \mathrm{g} / \mathrm{L}$ & Sample \\
\hline & Chicken Creek Downstream & $3 / 1 / 2007$ & $<1$ & $\mu \mathrm{g} / \mathrm{L}$ & Sample \\
\hline & Chicken Creek Upstream & $3 / 1 / 2007$ & $<1$ & $\mu \mathrm{g} / \mathrm{L}$ & Sample \\
\hline & $\begin{array}{l}\text { N. Fork Strawberry Creek } \\
\text { Downstream }\end{array}$ & 3/1/2007 & $<1$ & $\mu \mathrm{g} / \mathrm{L}$ & Sample \\
\hline & $\begin{array}{l}\text { N. Fork Strawberry Creek } \\
\text { Upstream }\end{array}$ & 3/1/2007 & $<1$ & $\mu \mathrm{g} / \mathrm{L}$ & Sample \\
\hline & No Name Creek & $3 / 1 / 2007$ & $<1$ & $\mu \mathrm{g} / \mathrm{L}$ & Sample \\
\hline & Ravine Creek & 3/1/2007 & $<1$ & $\mu \mathrm{g} / \mathrm{L}$ & Sample \\
\hline
\end{tabular}




\begin{tabular}{|c|c|c|c|c|c|}
\hline \multicolumn{2}{|c|}{ Volatile Organic Compounds } & \multirow[b]{2}{*}{$\begin{array}{c}\text { Collection } \\
\text { Date }\end{array}$} & \multirow[b]{2}{*}{ Result } & \multirow[b]{2}{*}{ Units } & \multirow[b]{2}{*}{$\begin{array}{c}\text { QA } \\
\text { Type }\end{array}$} \\
\hline Analyte & Location & & & & \\
\hline 1,1-Dichloropropene & Ten-Inch Creek & $3 / 1 / 2007$ & $<1$ & $\mu \mathrm{g} / \mathrm{L}$ & Sample \\
\hline \multirow[t]{9}{*}{ 1,2,3-Trichlorobenzene } & Botanical Garden Creek & $3 / 1 / 2007$ & $<2$ & $\mu \mathrm{g} / \mathrm{L}$ & Sample \\
\hline & Cafeteria Creek & $3 / 1 / 2007$ & $<2$ & $\mu \mathrm{g} / \mathrm{L}$ & Sample \\
\hline & Chicken Creek Downstream & $3 / 1 / 2007$ & $<2$ & $\mu \mathrm{g} / \mathrm{L}$ & Sample \\
\hline & Chicken Creek Upstream & $3 / 1 / 2007$ & $<2$ & $\mu \mathrm{g} / \mathrm{L}$ & Sample \\
\hline & $\begin{array}{l}\text { N. Fork Strawberry Creek } \\
\text { Downstream }\end{array}$ & $3 / 1 / 2007$ & $<2$ & $\mu \mathrm{g} / \mathrm{L}$ & Sample \\
\hline & $\begin{array}{l}\text { N. Fork Strawberry Creek } \\
\text { Upstream }\end{array}$ & 3/1/2007 & $<2$ & $\mu \mathrm{g} / \mathrm{L}$ & Sample \\
\hline & No Name Creek & $3 / 1 / 2007$ & $<2$ & $\mu \mathrm{g} / \mathrm{L}$ & Sample \\
\hline & Ravine Creek & $3 / 1 / 2007$ & $<2$ & $\mu \mathrm{g} / \mathrm{L}$ & Sample \\
\hline & Ten-Inch Creek & $3 / 1 / 2007$ & $<2$ & $\mu \mathrm{g} / \mathrm{L}$ & Sample \\
\hline \multirow[t]{9}{*}{ 1,2,3-Trichloropropane } & Botanical Garden Creek & 3/1/2007 & $<1$ & $\mu \mathrm{g} / \mathrm{L}$ & Sample \\
\hline & Cafeteria Creek & $3 / 1 / 2007$ & $<1$ & $\mu \mathrm{g} / \mathrm{L}$ & Sample \\
\hline & Chicken Creek Downstream & $3 / 1 / 2007$ & $<1$ & $\mu \mathrm{g} / \mathrm{L}$ & Sample \\
\hline & Chicken Creek Upstream & $3 / 1 / 2007$ & $<1$ & $\mu \mathrm{g} / \mathrm{L}$ & Sample \\
\hline & $\begin{array}{l}\text { N. Fork Strawberry Creek } \\
\text { Downstream }\end{array}$ & 3/1/2007 & $<1$ & $\mu \mathrm{g} / \mathrm{L}$ & Sample \\
\hline & $\begin{array}{l}\text { N. Fork Strawberry Creek } \\
\text { Upstream }\end{array}$ & 3/1/2007 & $<1$ & $\mu \mathrm{g} / \mathrm{L}$ & Sample \\
\hline & No Name Creek & 3/1/2007 & $<1$ & $\mu \mathrm{g} / \mathrm{L}$ & Sample \\
\hline & Ravine Creek & $3 / 1 / 2007$ & $<1$ & $\mu \mathrm{g} / \mathrm{L}$ & Sample \\
\hline & Ten-Inch Creek & $3 / 1 / 2007$ & $<1$ & $\mu \mathrm{g} / \mathrm{L}$ & Sample \\
\hline \multirow[t]{9}{*}{ 1,2,4-Trichlorobenzene } & Botanical Garden Creek & 3/1/2007 & $<1$ & $\mu \mathrm{g} / \mathrm{L}$ & Sample \\
\hline & Cafeteria Creek & $3 / 1 / 2007$ & $<1$ & $\mu \mathrm{g} / \mathrm{L}$ & Sample \\
\hline & Chicken Creek Downstream & $3 / 1 / 2007$ & $<1$ & $\mu \mathrm{g} / \mathrm{L}$ & Sample \\
\hline & Chicken Creek Upstream & $3 / 1 / 2007$ & $<1$ & $\mu \mathrm{g} / \mathrm{L}$ & Sample \\
\hline & $\begin{array}{l}\text { N. Fork Strawberry Creek } \\
\text { Downstream }\end{array}$ & $3 / 1 / 2007$ & $<1$ & $\mu \mathrm{g} / \mathrm{L}$ & Sample \\
\hline & $\begin{array}{l}\text { N. Fork Strawberry Creek } \\
\text { Upstream }\end{array}$ & 3/1/2007 & $<1$ & $\mu \mathrm{g} / \mathrm{L}$ & Sample \\
\hline & No Name Creek & $3 / 1 / 2007$ & $<1$ & $\mu \mathrm{g} / \mathrm{L}$ & Sample \\
\hline & Ravine Creek & $3 / 1 / 2007$ & $<1$ & $\mu \mathrm{g} / \mathrm{L}$ & Sample \\
\hline & Ten-Inch Creek & $3 / 1 / 2007$ & $<1$ & $\mu \mathrm{g} / \mathrm{L}$ & Sample \\
\hline \multirow[t]{4}{*}{ 1,2,4-Trimethylbenzene } & Botanical Garden Creek & 3/1/2007 & $<1$ & $\mu \mathrm{g} / \mathrm{L}$ & Sample \\
\hline & Cafeteria Creek & $3 / 1 / 2007$ & $<1$ & $\mu \mathrm{g} / \mathrm{L}$ & Sample \\
\hline & Chicken Creek Downstream & $3 / 1 / 2007$ & $<1$ & $\mu \mathrm{g} / \mathrm{L}$ & Sample \\
\hline & Chicken Creek Upstream & $3 / 1 / 2007$ & $<1$ & $\mu \mathrm{g} / \mathrm{L}$ & Sample \\
\hline
\end{tabular}




\begin{tabular}{|c|c|c|c|c|c|}
\hline \multicolumn{2}{|c|}{ Volatile Organic Compounds } & \multirow[b]{2}{*}{$\begin{array}{c}\text { Collection } \\
\text { Date }\end{array}$} & \multirow[b]{2}{*}{ Result } & \multirow[b]{2}{*}{ Units } & \multirow[b]{2}{*}{$\begin{array}{c}\text { QA } \\
\text { Type }\end{array}$} \\
\hline Analyte & Location & & & & \\
\hline \multirow[t]{5}{*}{ 1,2,4-Trimethylbenzene } & $\begin{array}{l}\text { N. Fork Strawberry Creek } \\
\text { Downstream }\end{array}$ & $3 / 1 / 2007$ & $<1$ & $\mu \mathrm{g} / \mathrm{L}$ & Sample \\
\hline & $\begin{array}{l}\text { N. Fork Strawberry Creek } \\
\text { Upstream }\end{array}$ & 3/1/2007 & $<1$ & $\mu \mathrm{g} / \mathrm{L}$ & Sample \\
\hline & No Name Creek & $3 / 1 / 2007$ & $<1$ & $\mu \mathrm{g} / \mathrm{L}$ & Sample \\
\hline & Ravine Creek & 3/1/2007 & $<1$ & $\mu \mathrm{g} / \mathrm{L}$ & Sample \\
\hline & Ten-Inch Creek & $3 / 1 / 2007$ & $<1$ & $\mu \mathrm{g} / \mathrm{L}$ & Sample \\
\hline \multirow[t]{9}{*}{$\begin{array}{l}\text { 1,2-Dibromo-3- } \\
\text { chloropropane }\end{array}$} & Botanical Garden Creek & $3 / 1 / 2007$ & $<2$ & $\mu \mathrm{g} / \mathrm{L}$ & Sample \\
\hline & Cafeteria Creek & $3 / 1 / 2007$ & $<2$ & $\mu \mathrm{g} / \mathrm{L}$ & Sample \\
\hline & Chicken Creek Downstream & $3 / 1 / 2007$ & $<2$ & $\mu \mathrm{g} / \mathrm{L}$ & Sample \\
\hline & Chicken Creek Upstream & $3 / 1 / 2007$ & $<2$ & $\mu \mathrm{g} / \mathrm{L}$ & Sample \\
\hline & $\begin{array}{l}\text { N. Fork Strawberry Creek } \\
\text { Downstream }\end{array}$ & $3 / 1 / 2007$ & $<2$ & $\mu \mathrm{g} / \mathrm{L}$ & Sample \\
\hline & $\begin{array}{l}\text { N. Fork Strawberry Creek } \\
\text { Upstream }\end{array}$ & $3 / 1 / 2007$ & $<2$ & $\mu \mathrm{g} / \mathrm{L}$ & Sample \\
\hline & No Name Creek & $3 / 1 / 2007$ & $<2$ & $\mu \mathrm{g} / \mathrm{L}$ & Sample \\
\hline & Ravine Creek & $3 / 1 / 2007$ & $<2$ & $\mu \mathrm{g} / \mathrm{L}$ & Sample \\
\hline & Ten-Inch Creek & 3/1/2007 & $<2$ & $\mu \mathrm{g} / \mathrm{L}$ & Sample \\
\hline \multirow[t]{9}{*}{ 1,2-Dichlorobenzene } & Botanical Garden Creek & $3 / 1 / 2007$ & $<1$ & $\mu \mathrm{g} / \mathrm{L}$ & Sample \\
\hline & Cafeteria Creek & $3 / 1 / 2007$ & $<1$ & $\mu \mathrm{g} / \mathrm{L}$ & Sample \\
\hline & Chicken Creek Downstream & $3 / 1 / 2007$ & $<1$ & $\mu \mathrm{g} / \mathrm{L}$ & Sample \\
\hline & Chicken Creek Upstream & $3 / 1 / 2007$ & $<1$ & $\mu \mathrm{g} / \mathrm{L}$ & Sample \\
\hline & $\begin{array}{l}\text { N. Fork Strawberry Creek } \\
\text { Downstream }\end{array}$ & $3 / 1 / 2007$ & $<1$ & $\mu \mathrm{g} / \mathrm{L}$ & Sample \\
\hline & $\begin{array}{l}\text { N. Fork Strawberry Creek } \\
\text { Upstream }\end{array}$ & $3 / 1 / 2007$ & $<1$ & $\mu \mathrm{g} / \mathrm{L}$ & Sample \\
\hline & No Name Creek & $3 / 1 / 2007$ & $<1$ & $\mu \mathrm{g} / \mathrm{L}$ & Sample \\
\hline & Ravine Creek & $3 / 1 / 2007$ & $<1$ & $\mu \mathrm{g} / \mathrm{L}$ & Sample \\
\hline & Ten-Inch Creek & $3 / 1 / 2007$ & $<1$ & $\mu \mathrm{g} / \mathrm{L}$ & Sample \\
\hline \multirow[t]{7}{*}{ 1,2-Dichloroethane } & Botanical Garden Creek & $3 / 1 / 2007$ & $<2$ & $\mu \mathrm{g} / \mathrm{L}$ & Sample \\
\hline & Cafeteria Creek & $3 / 1 / 2007$ & $<2$ & $\mu \mathrm{g} / \mathrm{L}$ & Sample \\
\hline & Chicken Creek Downstream & 3/1/2007 & $<2$ & $\mu \mathrm{g} / \mathrm{L}$ & Sample \\
\hline & Chicken Creek Upstream & 3/1/2007 & $<2$ & $\mu \mathrm{g} / \mathrm{L}$ & Sample \\
\hline & $\begin{array}{l}\text { N. Fork Strawberry Creek } \\
\text { Downstream }\end{array}$ & $3 / 1 / 2007$ & $<2$ & $\mu \mathrm{g} / \mathrm{L}$ & Sample \\
\hline & $\begin{array}{l}\text { N. Fork Strawberry Creek } \\
\text { Upstream }\end{array}$ & $3 / 1 / 2007$ & $<2$ & $\mu \mathrm{g} / \mathrm{L}$ & Sample \\
\hline & No Name Creek & $3 / 1 / 2007$ & $<2$ & $\mu \mathrm{g} / \mathrm{L}$ & Sample \\
\hline
\end{tabular}




\begin{tabular}{|c|c|c|c|c|c|}
\hline \multicolumn{2}{|c|}{ Volatile Organic Compounds } & \multirow[b]{2}{*}{$\begin{array}{c}\text { Collection } \\
\text { Date }\end{array}$} & \multirow[b]{2}{*}{ Result } & \multirow[b]{2}{*}{ Units } & \multirow[b]{2}{*}{$\begin{array}{c}\text { QA } \\
\text { Type }\end{array}$} \\
\hline Analyte & Location & & & & \\
\hline \multirow[t]{2}{*}{ 1,2-Dichloroethane } & Ravine Creek & $3 / 1 / 2007$ & $<2$ & $\mu \mathrm{g} / \mathrm{L}$ & Sample \\
\hline & Ten-Inch Creek & $3 / 1 / 2007$ & $<2$ & $\mu \mathrm{g} / \mathrm{L}$ & Sample \\
\hline \multirow[t]{9}{*}{ 1,2-Dichloropropane } & Botanical Garden Creek & $3 / 1 / 2007$ & $<1$ & $\mu g / L$ & Sample \\
\hline & Cafeteria Creek & $3 / 1 / 2007$ & $<1$ & $\mu \mathrm{g} / \mathrm{L}$ & Sample \\
\hline & Chicken Creek Downstream & $3 / 1 / 2007$ & $<1$ & $\mu \mathrm{g} / \mathrm{L}$ & Sample \\
\hline & Chicken Creek Upstream & $3 / 1 / 2007$ & $<1$ & $\mu \mathrm{g} / \mathrm{L}$ & Sample \\
\hline & $\begin{array}{l}\text { N. Fork Strawberry Creek } \\
\text { Downstream }\end{array}$ & 3/1/2007 & $<1$ & $\mu \mathrm{g} / \mathrm{L}$ & Sample \\
\hline & $\begin{array}{l}\text { N. Fork Strawberry Creek } \\
\text { Upstream }\end{array}$ & $3 / 1 / 2007$ & $<1$ & $\mu \mathrm{g} / \mathrm{L}$ & Sample \\
\hline & No Name Creek & $3 / 1 / 2007$ & $<1$ & $\mu \mathrm{g} / \mathrm{L}$ & Sample \\
\hline & Ravine Creek & $3 / 1 / 2007$ & $<1$ & $\mu \mathrm{g} / \mathrm{L}$ & Sample \\
\hline & Ten-Inch Creek & 3/1/2007 & $<1$ & $\mu \mathrm{g} / \mathrm{L}$ & Sample \\
\hline \multirow[t]{9}{*}{ 1,3-Dichlorobenzene } & Botanical Garden Creek & $3 / 1 / 2007$ & $<1$ & $\mu \mathrm{g} / \mathrm{L}$ & Sample \\
\hline & Cafeteria Creek & $3 / 1 / 2007$ & $<1$ & $\mu \mathrm{g} / \mathrm{L}$ & Sample \\
\hline & Chicken Creek Downstream & 3/1/2007 & $<1$ & $\mu \mathrm{g} / \mathrm{L}$ & Sample \\
\hline & Chicken Creek Upstream & $3 / 1 / 2007$ & $<1$ & $\mu \mathrm{g} / \mathrm{L}$ & Sample \\
\hline & $\begin{array}{l}\text { N. Fork Strawberry Creek } \\
\text { Downstream }\end{array}$ & 3/1/2007 & $<1$ & $\mu \mathrm{g} / \mathrm{L}$ & Sample \\
\hline & $\begin{array}{l}\text { N. Fork Strawberry Creek } \\
\text { Upstream }\end{array}$ & 3/1/2007 & $<1$ & $\mu \mathrm{g} / \mathrm{L}$ & Sample \\
\hline & No Name Creek & $3 / 1 / 2007$ & $<1$ & $\mu \mathrm{g} / \mathrm{L}$ & Sample \\
\hline & Ravine Creek & $3 / 1 / 2007$ & $<1$ & $\mu \mathrm{g} / \mathrm{L}$ & Sample \\
\hline & Ten-Inch Creek & 3/1/2007 & $<1$ & $\mu \mathrm{g} / \mathrm{L}$ & Sample \\
\hline \multirow[t]{9}{*}{ 1,3-Dichloropropane } & Botanical Garden Creek & $3 / 1 / 2007$ & $<1$ & $\mu \mathrm{g} / \mathrm{L}$ & Sample \\
\hline & Cafeteria Creek & $3 / 1 / 2007$ & $<1$ & $\mu \mathrm{g} / \mathrm{L}$ & Sample \\
\hline & Chicken Creek Downstream & 3/1/2007 & $<1$ & $\mu \mathrm{g} / \mathrm{L}$ & Sample \\
\hline & Chicken Creek Upstream & $3 / 1 / 2007$ & $<1$ & $\mu \mathrm{g} / \mathrm{L}$ & Sample \\
\hline & $\begin{array}{l}\text { N. Fork Strawberry Creek } \\
\text { Downstream }\end{array}$ & $3 / 1 / 2007$ & $<1$ & $\mu \mathrm{g} / \mathrm{L}$ & Sample \\
\hline & $\begin{array}{l}\text { N. Fork Strawberry Creek } \\
\text { Upstream }\end{array}$ & $3 / 1 / 2007$ & $<1$ & $\mu \mathrm{g} / \mathrm{L}$ & Sample \\
\hline & No Name Creek & $3 / 1 / 2007$ & $<1$ & $\mu \mathrm{g} / \mathrm{L}$ & Sample \\
\hline & Ravine Creek & $3 / 1 / 2007$ & $<1$ & $\mu \mathrm{g} / \mathrm{L}$ & Sample \\
\hline & Ten-Inch Creek & 3/1/2007 & $<1$ & $\mu \mathrm{g} / \mathrm{L}$ & Sample \\
\hline \multirow[t]{3}{*}{ 1,4-Dichlorobenzene } & Botanical Garden Creek & $3 / 1 / 2007$ & $<1$ & $\mu \mathrm{g} / \mathrm{L}$ & Sample \\
\hline & Cafeteria Creek & $3 / 1 / 2007$ & $<1$ & $\mu \mathrm{g} / \mathrm{L}$ & Sample \\
\hline & Chicken Creek Downstream & 3/1/2007 & $<1$ & $\mu \mathrm{g} / \mathrm{L}$ & Sample \\
\hline
\end{tabular}




\begin{tabular}{|c|c|c|c|c|c|}
\hline \multicolumn{2}{|c|}{ Volatile Organic Compounds } & \multirow[b]{2}{*}{$\begin{array}{c}\text { Collection } \\
\text { Date }\end{array}$} & \multirow[b]{2}{*}{ Result } & \multirow[b]{2}{*}{ Units } & \multirow[b]{2}{*}{$\begin{array}{c}\text { QA } \\
\text { Type }\end{array}$} \\
\hline Analyte & Location & & & & \\
\hline \multirow[t]{6}{*}{ 1,4-Dichlorobenzene } & Chicken Creek Upstream & $3 / 1 / 2007$ & $<1$ & $\mu \mathrm{g} / \mathrm{L}$ & Sample \\
\hline & $\begin{array}{l}\text { N. Fork Strawberry Creek } \\
\text { Downstream }\end{array}$ & $3 / 1 / 2007$ & $<1$ & $\mu \mathrm{g} / \mathrm{L}$ & Sample \\
\hline & $\begin{array}{l}\text { N. Fork Strawberry Creek } \\
\text { Upstream }\end{array}$ & $3 / 1 / 2007$ & $<1$ & $\mu \mathrm{g} / \mathrm{L}$ & Sample \\
\hline & No Name Creek & $3 / 1 / 2007$ & $<1$ & $\mu \mathrm{g} / \mathrm{L}$ & Sample \\
\hline & Ravine Creek & 3/1/2007 & $<1$ & $\mu \mathrm{g} / \mathrm{L}$ & Sample \\
\hline & Ten-Inch Creek & $3 / 1 / 2007$ & $<1$ & $\mu \mathrm{g} / \mathrm{L}$ & Sample \\
\hline \multirow[t]{9}{*}{ 2,2-Dichloropropane } & Botanical Garden Creek & 3/1/2007 & $<1$ & $\mu \mathrm{g} / \mathrm{L}$ & Sample \\
\hline & Cafeteria Creek & 3/1/2007 & $<1$ & $\mu \mathrm{g} / \mathrm{L}$ & Sample \\
\hline & Chicken Creek Downstream & $3 / 1 / 2007$ & $<1$ & $\mu \mathrm{g} / \mathrm{L}$ & Sample \\
\hline & Chicken Creek Upstream & 3/1/2007 & $<1$ & $\mu \mathrm{g} / \mathrm{L}$ & Sample \\
\hline & $\begin{array}{l}\text { N. Fork Strawberry Creek } \\
\text { Downstream }\end{array}$ & 3/1/2007 & $<1$ & $\mu \mathrm{g} / \mathrm{L}$ & Sample \\
\hline & $\begin{array}{l}\text { N. Fork Strawberry Creek } \\
\text { Upstream }\end{array}$ & 3/1/2007 & $<1$ & $\mu \mathrm{g} / \mathrm{L}$ & Sample \\
\hline & No Name Creek & 3/1/2007 & $<1$ & $\mu \mathrm{g} / \mathrm{L}$ & Sample \\
\hline & Ravine Creek & 3/1/2007 & $<1$ & $\mu \mathrm{g} / \mathrm{L}$ & Sample \\
\hline & Ten-Inch Creek & $3 / 1 / 2007$ & $<1$ & $\mu \mathrm{g} / \mathrm{L}$ & Sample \\
\hline \multirow[t]{9}{*}{ 2-Chlorotoluene } & Botanical Garden Creek & $3 / 1 / 2007$ & $<2$ & $\mu \mathrm{g} / \mathrm{L}$ & Sample \\
\hline & Cafeteria Creek & 3/1/2007 & $<2$ & $\mu \mathrm{g} / \mathrm{L}$ & Sample \\
\hline & Chicken Creek Downstream & $3 / 1 / 2007$ & $<2$ & $\mu \mathrm{g} / \mathrm{L}$ & Sample \\
\hline & Chicken Creek Upstream & $3 / 1 / 2007$ & $<2$ & $\mu \mathrm{g} / \mathrm{L}$ & Sample \\
\hline & $\begin{array}{l}\text { N. Fork Strawberry Creek } \\
\text { Downstream }\end{array}$ & 3/1/2007 & $<2$ & $\mu \mathrm{g} / \mathrm{L}$ & Sample \\
\hline & $\begin{array}{l}\text { N. Fork Strawberry Creek } \\
\text { Upstream }\end{array}$ & $3 / 1 / 2007$ & $<2$ & $\mu \mathrm{g} / \mathrm{L}$ & Sample \\
\hline & No Name Creek & 3/1/2007 & $<2$ & $\mu \mathrm{g} / \mathrm{L}$ & Sample \\
\hline & Ravine Creek & 3/1/2007 & $<2$ & $\mu \mathrm{g} / \mathrm{L}$ & Sample \\
\hline & Ten-Inch Creek & 3/1/2007 & $<2$ & $\mu \mathrm{g} / \mathrm{L}$ & Sample \\
\hline \multirow[t]{7}{*}{ 4-Chlorotoluene } & Botanical Garden Creek & $3 / 1 / 2007$ & $<2$ & $\mu \mathrm{g} / \mathrm{L}$ & Sample \\
\hline & Cafeteria Creek & 3/1/2007 & $<2$ & $\mu g / L$ & Sample \\
\hline & Chicken Creek Downstream & 3/1/2007 & $<2$ & $\mu \mathrm{g} / \mathrm{L}$ & Sample \\
\hline & Chicken Creek Upstream & $3 / 1 / 2007$ & $<2$ & $\mu \mathrm{g} / \mathrm{L}$ & Sample \\
\hline & $\begin{array}{l}\text { N. Fork Strawberry Creek } \\
\text { Downstream }\end{array}$ & 3/1/2007 & $<2$ & $\mu \mathrm{g} / \mathrm{L}$ & Sample \\
\hline & $\begin{array}{l}\text { N. Fork Strawberry Creek } \\
\text { Upstream }\end{array}$ & $3 / 1 / 2007$ & $<2$ & $\mu \mathrm{g} / \mathrm{L}$ & Sample \\
\hline & No Name Creek & $3 / 1 / 2007$ & $<2$ & $\mu \mathrm{g} / \mathrm{L}$ & Sample \\
\hline
\end{tabular}




\begin{tabular}{|c|c|c|c|c|c|}
\hline \multicolumn{2}{|c|}{ Volatile Organic Compounds } & \multirow[b]{2}{*}{$\begin{array}{l}\text { Collection } \\
\text { Date }\end{array}$} & \multirow[b]{2}{*}{ Result } & \multirow[b]{2}{*}{ Units } & \multirow[b]{2}{*}{$\begin{array}{c}\text { QA } \\
\text { Type }\end{array}$} \\
\hline Analyte & Location & & & & \\
\hline \multirow[t]{2}{*}{ 4-Chlorotoluene } & Ravine Creek & $3 / 1 / 2007$ & $<2$ & $\mu g / L$ & Sample \\
\hline & Ten-Inch Creek & $3 / 1 / 2007$ & $<2$ & $\mu \mathrm{g} / \mathrm{L}$ & Sample \\
\hline \multirow[t]{9}{*}{ Benzene } & Botanical Garden Creek & $3 / 1 / 2007$ & $<1$ & $\mu g / L$ & Sample \\
\hline & Cafeteria Creek & $3 / 1 / 2007$ & $<1$ & $\mu g / L$ & Sample \\
\hline & Chicken Creek Downstream & $3 / 1 / 2007$ & $<1$ & $\mu g / L$ & Sample \\
\hline & Chicken Creek Upstream & $3 / 1 / 2007$ & $<1$ & $\mu \mathrm{g} / \mathrm{L}$ & Sample \\
\hline & $\begin{array}{l}\text { N. Fork Strawberry Creek } \\
\text { Downstream }\end{array}$ & $3 / 1 / 2007$ & $<1$ & $\mu \mathrm{g} / \mathrm{L}$ & Sample \\
\hline & $\begin{array}{l}\text { N. Fork Strawberry Creek } \\
\text { Upstream }\end{array}$ & $3 / 1 / 2007$ & $<1$ & $\mu g / L$ & Sample \\
\hline & No Name Creek & $3 / 1 / 2007$ & $<1$ & $\mu g / L$ & Sample \\
\hline & Ravine Creek & $3 / 1 / 2007$ & $<1$ & $\mu g / L$ & Sample \\
\hline & Ten-Inch Creek & $3 / 1 / 2007$ & $<1$ & $\mu \mathrm{g} / \mathrm{L}$ & Sample \\
\hline \multirow[t]{9}{*}{ Bromobenzene } & Botanical Garden Creek & $3 / 1 / 2007$ & $<1$ & $\mu g / L$ & Sample \\
\hline & Cafeteria Creek & $3 / 1 / 2007$ & $<1$ & $\mu g / L$ & Sample \\
\hline & Chicken Creek Downstream & $3 / 1 / 2007$ & $<1$ & $\mu \mathrm{g} / \mathrm{L}$ & Sample \\
\hline & Chicken Creek Upstream & $3 / 1 / 2007$ & $<1$ & $\mu g / L$ & Sample \\
\hline & $\begin{array}{l}\text { N. Fork Strawberry Creek } \\
\text { Downstream }\end{array}$ & $3 / 1 / 2007$ & $<1$ & $\mu \mathrm{g} / \mathrm{L}$ & Sample \\
\hline & $\begin{array}{l}\text { N. Fork Strawberry Creek } \\
\text { Upstream }\end{array}$ & $3 / 1 / 2007$ & $<1$ & $\mu \mathrm{g} / \mathrm{L}$ & Sample \\
\hline & No Name Creek & $3 / 1 / 2007$ & $<1$ & $\mu g / L$ & Sample \\
\hline & Ravine Creek & $3 / 1 / 2007$ & $<1$ & $\mu g / L$ & Sample \\
\hline & Ten-Inch Creek & $3 / 1 / 2007$ & $<1$ & $\mu \mathrm{g} / \mathrm{L}$ & Sample \\
\hline \multirow[t]{9}{*}{ Bromochloromethane } & Botanical Garden Creek & $3 / 1 / 2007$ & $<2$ & $\mu g / L$ & Sample \\
\hline & Cafeteria Creek & $3 / 1 / 2007$ & $<2$ & $\mu \mathrm{g} / \mathrm{L}$ & Sample \\
\hline & Chicken Creek Downstream & $3 / 1 / 2007$ & $<2$ & $\mu g / L$ & Sample \\
\hline & Chicken Creek Upstream & $3 / 1 / 2007$ & $<2$ & $\mu g / L$ & Sample \\
\hline & $\begin{array}{l}\text { N. Fork Strawberry Creek } \\
\text { Downstream }\end{array}$ & $3 / 1 / 2007$ & $<2$ & $\mu \mathrm{g} / \mathrm{L}$ & Sample \\
\hline & $\begin{array}{l}\text { N. Fork Strawberry Creek } \\
\text { Upstream }\end{array}$ & $3 / 1 / 2007$ & $<2$ & $\mu g / L$ & Sample \\
\hline & No Name Creek & $3 / 1 / 2007$ & $<2$ & $\mu \mathrm{g} / \mathrm{L}$ & Sample \\
\hline & Ravine Creek & $3 / 1 / 2007$ & $<2$ & $\mu \mathrm{g} / \mathrm{L}$ & Sample \\
\hline & Ten-Inch Creek & $3 / 1 / 2007$ & $<2$ & $\mu \mathrm{g} / \mathrm{L}$ & Sample \\
\hline \multirow[t]{3}{*}{ Bromodichloromethane } & Botanical Garden Creek & $3 / 1 / 2007$ & $<1$ & $\mu g / L$ & Sample \\
\hline & Cafeteria Creek & $3 / 1 / 2007$ & $<1$ & $\mu g / L$ & Sample \\
\hline & Chicken Creek Downstream & $3 / 1 / 2007$ & $<1$ & $\mu \mathrm{g} / \mathrm{L}$ & Sample \\
\hline
\end{tabular}




\begin{tabular}{|c|c|c|c|c|c|}
\hline \multicolumn{2}{|c|}{ Volatile Organic Compounds } & \multirow[b]{2}{*}{$\begin{array}{c}\text { Collection } \\
\text { Date }\end{array}$} & \multirow[b]{2}{*}{ Result } & \multirow[b]{2}{*}{ Units } & \multirow[b]{2}{*}{$\begin{array}{c}\text { QA } \\
\text { Type }\end{array}$} \\
\hline Analyte & Location & & & & \\
\hline \multirow[t]{6}{*}{ Bromodichloromethane } & Chicken Creek Upstream & $3 / 1 / 2007$ & $<1$ & $\mu \mathrm{g} / \mathrm{L}$ & Sample \\
\hline & $\begin{array}{l}\text { N. Fork Strawberry Creek } \\
\text { Downstream }\end{array}$ & $3 / 1 / 2007$ & $<1$ & $\mu \mathrm{g} / \mathrm{L}$ & Sample \\
\hline & $\begin{array}{l}\text { N. Fork Strawberry Creek } \\
\text { Upstream }\end{array}$ & $3 / 1 / 2007$ & $<1$ & $\mu \mathrm{g} / \mathrm{L}$ & Sample \\
\hline & No Name Creek & $3 / 1 / 2007$ & $<1$ & $\mu \mathrm{g} / \mathrm{L}$ & Sample \\
\hline & Ravine Creek & 3/1/2007 & $<1$ & $\mu \mathrm{g} / \mathrm{L}$ & Sample \\
\hline & Ten-Inch Creek & $3 / 1 / 2007$ & $<1$ & $\mu \mathrm{g} / \mathrm{L}$ & Sample \\
\hline \multirow[t]{9}{*}{ Bromoform } & Botanical Garden Creek & 3/1/2007 & $<2$ & $\mu \mathrm{g} / \mathrm{L}$ & Sample \\
\hline & Cafeteria Creek & 3/1/2007 & $<2$ & $\mu \mathrm{g} / \mathrm{L}$ & Sample \\
\hline & Chicken Creek Downstream & $3 / 1 / 2007$ & $<2$ & $\mu \mathrm{g} / \mathrm{L}$ & Sample \\
\hline & Chicken Creek Upstream & 3/1/2007 & $<2$ & $\mu \mathrm{g} / \mathrm{L}$ & Sample \\
\hline & $\begin{array}{l}\text { N. Fork Strawberry Creek } \\
\text { Downstream }\end{array}$ & 3/1/2007 & $<2$ & $\mu \mathrm{g} / \mathrm{L}$ & Sample \\
\hline & $\begin{array}{l}\text { N. Fork Strawberry Creek } \\
\text { Upstream }\end{array}$ & 3/1/2007 & $<2$ & $\mu \mathrm{g} / \mathrm{L}$ & Sample \\
\hline & No Name Creek & 3/1/2007 & $<2$ & $\mu \mathrm{g} / \mathrm{L}$ & Sample \\
\hline & Ravine Creek & 3/1/2007 & $<2$ & $\mu \mathrm{g} / \mathrm{L}$ & Sample \\
\hline & Ten-Inch Creek & $3 / 1 / 2007$ & $<2$ & $\mu \mathrm{g} / \mathrm{L}$ & Sample \\
\hline \multirow[t]{9}{*}{ Bromomethane } & Botanical Garden Creek & $3 / 1 / 2007$ & $<10$ & $\mu \mathrm{g} / \mathrm{L}$ & Sample \\
\hline & Cafeteria Creek & 3/1/2007 & $<10$ & $\mu \mathrm{g} / \mathrm{L}$ & Sample \\
\hline & Chicken Creek Downstream & $3 / 1 / 2007$ & $<10$ & $\mu \mathrm{g} / \mathrm{L}$ & Sample \\
\hline & Chicken Creek Upstream & $3 / 1 / 2007$ & $<10$ & $\mu \mathrm{g} / \mathrm{L}$ & Sample \\
\hline & $\begin{array}{l}\text { N. Fork Strawberry Creek } \\
\text { Downstream }\end{array}$ & 3/1/2007 & $<10$ & $\mu \mathrm{g} / \mathrm{L}$ & Sample \\
\hline & $\begin{array}{l}\text { N. Fork Strawberry Creek } \\
\text { Upstream }\end{array}$ & $3 / 1 / 2007$ & $<10$ & $\mu \mathrm{g} / \mathrm{L}$ & Sample \\
\hline & No Name Creek & 3/1/2007 & $<10$ & $\mu \mathrm{g} / \mathrm{L}$ & Sample \\
\hline & Ravine Creek & 3/1/2007 & $<10$ & $\mu \mathrm{g} / \mathrm{L}$ & Sample \\
\hline & Ten-Inch Creek & 3/1/2007 & $<10$ & $\mu \mathrm{g} / \mathrm{L}$ & Sample \\
\hline \multirow[t]{7}{*}{ Carbon tetrachloride } & Botanical Garden Creek & $3 / 1 / 2007$ & $<1$ & $\mu \mathrm{g} / \mathrm{L}$ & Sample \\
\hline & Cafeteria Creek & 3/1/2007 & $<1$ & $\mu g / L$ & Sample \\
\hline & Chicken Creek Downstream & 3/1/2007 & $<1$ & $\mu \mathrm{g} / \mathrm{L}$ & Sample \\
\hline & Chicken Creek Upstream & $3 / 1 / 2007$ & $<1$ & $\mu \mathrm{g} / \mathrm{L}$ & Sample \\
\hline & $\begin{array}{l}\text { N. Fork Strawberry Creek } \\
\text { Downstream }\end{array}$ & 3/1/2007 & $<1$ & $\mu \mathrm{g} / \mathrm{L}$ & Sample \\
\hline & $\begin{array}{l}\text { N. Fork Strawberry Creek } \\
\text { Upstream }\end{array}$ & $3 / 1 / 2007$ & $<1$ & $\mu \mathrm{g} / \mathrm{L}$ & Sample \\
\hline & No Name Creek & $3 / 1 / 2007$ & $<1$ & $\mu \mathrm{g} / \mathrm{L}$ & Sample \\
\hline
\end{tabular}




\begin{tabular}{|c|c|c|c|c|c|}
\hline \multicolumn{2}{|c|}{ Volatile Organic Compounds } & \multirow[b]{2}{*}{$\begin{array}{c}\text { Collection } \\
\text { Date }\end{array}$} & \multirow[b]{2}{*}{ Result } & \multirow[b]{2}{*}{ Units } & \multirow[b]{2}{*}{$\begin{array}{c}\text { QA } \\
\text { Type }\end{array}$} \\
\hline Analyte & Location & & & & \\
\hline \multirow[t]{2}{*}{ Carbon tetrachloride } & Ravine Creek & $3 / 1 / 2007$ & $<1$ & $\mu g / L$ & Sample \\
\hline & Ten-Inch Creek & $3 / 1 / 2007$ & $<1$ & $\mu \mathrm{g} / \mathrm{L}$ & Sample \\
\hline \multirow[t]{9}{*}{ Chlorobenzene } & Botanical Garden Creek & $3 / 1 / 2007$ & $<1$ & $\mu g / L$ & Sample \\
\hline & Cafeteria Creek & $3 / 1 / 2007$ & $<1$ & $\mu \mathrm{g} / \mathrm{L}$ & Sample \\
\hline & Chicken Creek Downstream & $3 / 1 / 2007$ & $<1$ & $\mu \mathrm{g} / \mathrm{L}$ & Sample \\
\hline & Chicken Creek Upstream & $3 / 1 / 2007$ & $<1$ & $\mu \mathrm{g} / \mathrm{L}$ & Sample \\
\hline & $\begin{array}{l}\text { N. Fork Strawberry Creek } \\
\text { Downstream }\end{array}$ & $3 / 1 / 2007$ & $<1$ & $\mu g / L$ & Sample \\
\hline & $\begin{array}{l}\text { N. Fork Strawberry Creek } \\
\text { Upstream }\end{array}$ & $3 / 1 / 2007$ & $<1$ & $\mu g / L$ & Sample \\
\hline & No Name Creek & $3 / 1 / 2007$ & $<1$ & $\mu \mathrm{g} / \mathrm{L}$ & Sample \\
\hline & Ravine Creek & $3 / 1 / 2007$ & $<1$ & $\mu \mathrm{g} / \mathrm{L}$ & Sample \\
\hline & Ten-Inch Creek & $3 / 1 / 2007$ & $<1$ & $\mu g / L$ & Sample \\
\hline \multirow[t]{9}{*}{ Chlorodifluoromethane } & Botanical Garden Creek & $3 / 1 / 2007$ & $<30$ & $\mu \mathrm{g} / \mathrm{L}$ & Sample \\
\hline & Cafeteria Creek & $3 / 1 / 2007$ & $<30$ & $\mu \mathrm{g} / \mathrm{L}$ & Sample \\
\hline & Chicken Creek Downstream & $3 / 1 / 2007$ & $<30$ & $\mu g / L$ & Sample \\
\hline & Chicken Creek Upstream & $3 / 1 / 2007$ & $<30$ & $\mu \mathrm{g} / \mathrm{L}$ & Sample \\
\hline & $\begin{array}{l}\text { N. Fork Strawberry Creek } \\
\text { Downstream }\end{array}$ & $3 / 1 / 2007$ & $<30$ & $\mu \mathrm{g} / \mathrm{L}$ & Sample \\
\hline & $\begin{array}{l}\text { N. Fork Strawberry Creek } \\
\text { Upstream }\end{array}$ & $3 / 1 / 2007$ & $<30$ & $\mu \mathrm{g} / \mathrm{L}$ & Sample \\
\hline & No Name Creek & $3 / 1 / 2007$ & $<30$ & $\mu \mathrm{g} / \mathrm{L}$ & Sample \\
\hline & Ravine Creek & $3 / 1 / 2007$ & $<30$ & $\mu \mathrm{g} / \mathrm{L}$ & Sample \\
\hline & Ten-Inch Creek & $3 / 1 / 2007$ & $<30$ & $\mu g / L$ & Sample \\
\hline \multirow[t]{9}{*}{ Chloroethane } & Botanical Garden Creek & $3 / 1 / 2007$ & $<30$ & $\mu g / L$ & Sample \\
\hline & Cafeteria Creek & $3 / 1 / 2007$ & $<30$ & $\mu \mathrm{g} / \mathrm{L}$ & Sample \\
\hline & Chicken Creek Downstream & $3 / 1 / 2007$ & $<30$ & $\mu \mathrm{g} / \mathrm{L}$ & Sample \\
\hline & Chicken Creek Upstream & $3 / 1 / 2007$ & $<30$ & $\mu \mathrm{g} / \mathrm{L}$ & Sample \\
\hline & $\begin{array}{l}\text { N. Fork Strawberry Creek } \\
\text { Downstream }\end{array}$ & $3 / 1 / 2007$ & $<30$ & $\mu g / L$ & Sample \\
\hline & $\begin{array}{l}\text { N. Fork Strawberry Creek } \\
\text { Upstream }\end{array}$ & $3 / 1 / 2007$ & $<30$ & $\mu g / L$ & Sample \\
\hline & No Name Creek & $3 / 1 / 2007$ & $<30$ & $\mu g / L$ & Sample \\
\hline & Ravine Creek & $3 / 1 / 2007$ & $<30$ & $\mu \mathrm{g} / \mathrm{L}$ & Sample \\
\hline & Ten-Inch Creek & $3 / 1 / 2007$ & $<30$ & $\mu \mathrm{g} / \mathrm{L}$ & Sample \\
\hline \multirow[t]{3}{*}{ Chloroform } & Botanical Garden Creek & $3 / 1 / 2007$ & $<3$ & $\mu \mathrm{g} / \mathrm{L}$ & Sample \\
\hline & Cafeteria Creek & $3 / 1 / 2007$ & $<3$ & $\mu \mathrm{g} / \mathrm{L}$ & Sample \\
\hline & Chicken Creek Downstream & $3 / 1 / 2007$ & $<3$ & $\mu \mathrm{g} / \mathrm{L}$ & Sample \\
\hline
\end{tabular}




\begin{tabular}{|c|c|c|c|c|c|}
\hline \multicolumn{2}{|c|}{ Volatile Organic Compounds } & \multirow[b]{2}{*}{$\begin{array}{c}\text { Collection } \\
\text { Date }\end{array}$} & \multirow[b]{2}{*}{ Result } & \multirow[b]{2}{*}{ Units } & \multirow[b]{2}{*}{$\begin{array}{c}\text { QA } \\
\text { Type }\end{array}$} \\
\hline Analyte & Location & & & & \\
\hline \multirow[t]{6}{*}{ Chloroform } & Chicken Creek Upstream & $3 / 1 / 2007$ & $<3$ & $\mu \mathrm{g} / \mathrm{L}$ & Sample \\
\hline & $\begin{array}{l}\text { N. Fork Strawberry Creek } \\
\text { Downstream }\end{array}$ & $3 / 1 / 2007$ & $<3$ & $\mu \mathrm{g} / \mathrm{L}$ & Sample \\
\hline & $\begin{array}{l}\text { N. Fork Strawberry Creek } \\
\text { Upstream }\end{array}$ & $3 / 1 / 2007$ & $<3$ & $\mu \mathrm{g} / \mathrm{L}$ & Sample \\
\hline & No Name Creek & $3 / 1 / 2007$ & $<3$ & $\mu \mathrm{g} / \mathrm{L}$ & Sample \\
\hline & Ravine Creek & $3 / 1 / 2007$ & $<3$ & $\mu \mathrm{g} / \mathrm{L}$ & Sample \\
\hline & Ten-Inch Creek & $3 / 1 / 2007$ & $<3$ & $\mu \mathrm{g} / \mathrm{L}$ & Sample \\
\hline \multirow[t]{9}{*}{ Chloromethane } & Botanical Garden Creek & 3/1/2007 & $<10$ & $\mu \mathrm{g} / \mathrm{L}$ & Sample \\
\hline & Cafeteria Creek & 3/1/2007 & $<10$ & $\mu \mathrm{g} / \mathrm{L}$ & Sample \\
\hline & Chicken Creek Downstream & $3 / 1 / 2007$ & $<10$ & $\mu \mathrm{g} / \mathrm{L}$ & Sample \\
\hline & Chicken Creek Upstream & 3/1/2007 & $<10$ & $\mu \mathrm{g} / \mathrm{L}$ & Sample \\
\hline & $\begin{array}{l}\text { N. Fork Strawberry Creek } \\
\text { Downstream }\end{array}$ & 3/1/2007 & $<10$ & $\mu \mathrm{g} / \mathrm{L}$ & Sample \\
\hline & $\begin{array}{l}\text { N. Fork Strawberry Creek } \\
\text { Upstream }\end{array}$ & 3/1/2007 & $<10$ & $\mu \mathrm{g} / \mathrm{L}$ & Sample \\
\hline & No Name Creek & 3/1/2007 & $<10$ & $\mu \mathrm{g} / \mathrm{L}$ & Sample \\
\hline & Ravine Creek & 3/1/2007 & $<10$ & $\mu \mathrm{g} / \mathrm{L}$ & Sample \\
\hline & Ten-Inch Creek & $3 / 1 / 2007$ & $<10$ & $\mu \mathrm{g} / \mathrm{L}$ & Sample \\
\hline \multirow[t]{9}{*}{ cis-1,2-Dichloroethene } & Botanical Garden Creek & $3 / 1 / 2007$ & $<1$ & $\mu \mathrm{g} / \mathrm{L}$ & Sample \\
\hline & Cafeteria Creek & 3/1/2007 & $<1$ & $\mu \mathrm{g} / \mathrm{L}$ & Sample \\
\hline & Chicken Creek Downstream & $3 / 1 / 2007$ & $<1$ & $\mu \mathrm{g} / \mathrm{L}$ & Sample \\
\hline & Chicken Creek Upstream & $3 / 1 / 2007$ & $<1$ & $\mu \mathrm{g} / \mathrm{L}$ & Sample \\
\hline & $\begin{array}{l}\text { N. Fork Strawberry Creek } \\
\text { Downstream }\end{array}$ & 3/1/2007 & $<1$ & $\mu \mathrm{g} / \mathrm{L}$ & Sample \\
\hline & $\begin{array}{l}\text { N. Fork Strawberry Creek } \\
\text { Upstream }\end{array}$ & $3 / 1 / 2007$ & $<1$ & $\mu \mathrm{g} / \mathrm{L}$ & Sample \\
\hline & No Name Creek & 3/1/2007 & $<1$ & $\mu \mathrm{g} / \mathrm{L}$ & Sample \\
\hline & Ravine Creek & 3/1/2007 & $<1$ & $\mu \mathrm{g} / \mathrm{L}$ & Sample \\
\hline & Ten-Inch Creek & 3/1/2007 & $<1$ & $\mu \mathrm{g} / \mathrm{L}$ & Sample \\
\hline \multirow[t]{7}{*}{ cis-1,3-Dichloropropene } & Botanical Garden Creek & $3 / 1 / 2007$ & $<1$ & $\mu \mathrm{g} / \mathrm{L}$ & Sample \\
\hline & Cafeteria Creek & 3/1/2007 & $<1$ & $\mu g / L$ & Sample \\
\hline & Chicken Creek Downstream & 3/1/2007 & $<1$ & $\mu \mathrm{g} / \mathrm{L}$ & Sample \\
\hline & Chicken Creek Upstream & $3 / 1 / 2007$ & $<1$ & $\mu \mathrm{g} / \mathrm{L}$ & Sample \\
\hline & $\begin{array}{l}\text { N. Fork Strawberry Creek } \\
\text { Downstream }\end{array}$ & 3/1/2007 & $<1$ & $\mu \mathrm{g} / \mathrm{L}$ & Sample \\
\hline & $\begin{array}{l}\text { N. Fork Strawberry Creek } \\
\text { Upstream }\end{array}$ & $3 / 1 / 2007$ & $<1$ & $\mu \mathrm{g} / \mathrm{L}$ & Sample \\
\hline & No Name Creek & $3 / 1 / 2007$ & $<1$ & $\mu \mathrm{g} / \mathrm{L}$ & Sample \\
\hline
\end{tabular}




\begin{tabular}{|c|c|c|c|c|c|}
\hline \multicolumn{2}{|c|}{ Volatile Organic Compounds } & \multirow[b]{2}{*}{$\begin{array}{c}\text { Collection } \\
\text { Date }\end{array}$} & \multirow[b]{2}{*}{ Result } & \multirow[b]{2}{*}{ Units } & \multirow[b]{2}{*}{$\begin{array}{c}\text { QA } \\
\text { Type }\end{array}$} \\
\hline Analyte & Location & & & & \\
\hline \multirow[t]{2}{*}{ cis-1,3-Dichloropropene } & Ravine Creek & $3 / 1 / 2007$ & $<1$ & $\mu \mathrm{g} / \mathrm{L}$ & Sample \\
\hline & Ten-Inch Creek & $3 / 1 / 2007$ & $<1$ & $\mu \mathrm{g} / \mathrm{L}$ & Sample \\
\hline \multirow[t]{9}{*}{ Dibromochloromethane } & Botanical Garden Creek & $3 / 1 / 2007$ & $<2$ & $\mu g / L$ & Sample \\
\hline & Cafeteria Creek & $3 / 1 / 2007$ & $<2$ & $\mu \mathrm{g} / \mathrm{L}$ & Sample \\
\hline & Chicken Creek Downstream & $3 / 1 / 2007$ & $<2$ & $\mu \mathrm{g} / \mathrm{L}$ & Sample \\
\hline & Chicken Creek Upstream & $3 / 1 / 2007$ & $<2$ & $\mu \mathrm{g} / \mathrm{L}$ & Sample \\
\hline & $\begin{array}{l}\text { N. Fork Strawberry Creek } \\
\text { Downstream }\end{array}$ & $3 / 1 / 2007$ & $<2$ & $\mu g / L$ & Sample \\
\hline & $\begin{array}{l}\text { N. Fork Strawberry Creek } \\
\text { Upstream }\end{array}$ & $3 / 1 / 2007$ & $<2$ & $\mu g / L$ & Sample \\
\hline & No Name Creek & $3 / 1 / 2007$ & $<2$ & $\mu \mathrm{g} / \mathrm{L}$ & Sample \\
\hline & Ravine Creek & $3 / 1 / 2007$ & $<2$ & $\mu \mathrm{g} / \mathrm{L}$ & Sample \\
\hline & Ten-Inch Creek & $3 / 1 / 2007$ & $<2$ & $\mu g / L$ & Sample \\
\hline \multirow[t]{9}{*}{ Dichlorodifluoromethane } & Botanical Garden Creek & $3 / 1 / 2007$ & $<3$ & $\mu \mathrm{g} / \mathrm{L}$ & Sample \\
\hline & Cafeteria Creek & $3 / 1 / 2007$ & $<3$ & $\mu \mathrm{g} / \mathrm{L}$ & Sample \\
\hline & Chicken Creek Downstream & $3 / 1 / 2007$ & $<3$ & $\mu g / L$ & Sample \\
\hline & Chicken Creek Upstream & $3 / 1 / 2007$ & $<3$ & $\mu \mathrm{g} / \mathrm{L}$ & Sample \\
\hline & $\begin{array}{l}\text { N. Fork Strawberry Creek } \\
\text { Downstream }\end{array}$ & 3/1/2007 & $<3$ & $\mu \mathrm{g} / \mathrm{L}$ & Sample \\
\hline & $\begin{array}{l}\text { N. Fork Strawberry Creek } \\
\text { Upstream }\end{array}$ & $3 / 1 / 2007$ & $<3$ & $\mu \mathrm{g} / \mathrm{L}$ & Sample \\
\hline & No Name Creek & $3 / 1 / 2007$ & $<3$ & $\mu \mathrm{g} / \mathrm{L}$ & Sample \\
\hline & Ravine Creek & $3 / 1 / 2007$ & $<3$ & $\mu \mathrm{g} / \mathrm{L}$ & Sample \\
\hline & Ten-Inch Creek & $3 / 1 / 2007$ & $<3$ & $\mu g / L$ & Sample \\
\hline \multirow[t]{9}{*}{ Dichlorotrifluoroethane } & Botanical Garden Creek & $3 / 1 / 2007$ & $<1$ & $\mu g / L$ & Sample \\
\hline & Cafeteria Creek & $3 / 1 / 2007$ & $<1$ & $\mu g / L$ & Sample \\
\hline & Chicken Creek Downstream & $3 / 1 / 2007$ & $<1$ & $\mu \mathrm{g} / \mathrm{L}$ & Sample \\
\hline & Chicken Creek Upstream & $3 / 1 / 2007$ & $<1$ & $\mu \mathrm{g} / \mathrm{L}$ & Sample \\
\hline & $\begin{array}{l}\text { N. Fork Strawberry Creek } \\
\text { Downstream }\end{array}$ & $3 / 1 / 2007$ & $<1$ & $\mu g / L$ & Sample \\
\hline & $\begin{array}{l}\text { N. Fork Strawberry Creek } \\
\text { Upstream }\end{array}$ & $3 / 1 / 2007$ & $<1$ & $\mu g / L$ & Sample \\
\hline & No Name Creek & $3 / 1 / 2007$ & $<1$ & $\mu g / L$ & Sample \\
\hline & Ravine Creek & $3 / 1 / 2007$ & $<1$ & $\mu \mathrm{g} / \mathrm{L}$ & Sample \\
\hline & Ten-Inch Creek & $3 / 1 / 2007$ & $<1$ & $\mu \mathrm{g} / \mathrm{L}$ & Sample \\
\hline \multirow[t]{3}{*}{ Ethylbenzene } & Botanical Garden Creek & $3 / 1 / 2007$ & $<1$ & $\mu \mathrm{g} / \mathrm{L}$ & Sample \\
\hline & Cafeteria Creek & $3 / 1 / 2007$ & $<1$ & $\mu \mathrm{g} / \mathrm{L}$ & Sample \\
\hline & Chicken Creek Downstream & $3 / 1 / 2007$ & $<1$ & $\mu \mathrm{g} / \mathrm{L}$ & Sample \\
\hline
\end{tabular}




\begin{tabular}{|c|c|c|c|c|c|}
\hline \multicolumn{2}{|c|}{ Volatile Organic Compounds } & \multirow[b]{2}{*}{$\begin{array}{c}\text { Collection } \\
\text { Date }\end{array}$} & \multirow[b]{2}{*}{ Result } & \multirow[b]{2}{*}{ Units } & \multirow[b]{2}{*}{$\begin{array}{c}\text { QA } \\
\text { Type }\end{array}$} \\
\hline Analyte & Location & & & & \\
\hline \multirow[t]{6}{*}{ Ethylbenzene } & Chicken Creek Upstream & $3 / 1 / 2007$ & $<1$ & $\mu \mathrm{g} / \mathrm{L}$ & Sample \\
\hline & $\begin{array}{l}\text { N. Fork Strawberry Creek } \\
\text { Downstream }\end{array}$ & $3 / 1 / 2007$ & $<1$ & $\mu \mathrm{g} / \mathrm{L}$ & Sample \\
\hline & $\begin{array}{l}\text { N. Fork Strawberry Creek } \\
\text { Upstream }\end{array}$ & $3 / 1 / 2007$ & $<1$ & $\mu \mathrm{g} / \mathrm{L}$ & Sample \\
\hline & No Name Creek & $3 / 1 / 2007$ & $<1$ & $\mu \mathrm{g} / \mathrm{L}$ & Sample \\
\hline & Ravine Creek & $3 / 1 / 2007$ & $<1$ & $\mu \mathrm{g} / \mathrm{L}$ & Sample \\
\hline & Ten-Inch Creek & $3 / 1 / 2007$ & $<1$ & $\mu \mathrm{g} / \mathrm{L}$ & Sample \\
\hline \multirow[t]{9}{*}{ Ethylene Dibromide } & Botanical Garden Creek & 3/1/2007 & $<2$ & $\mu \mathrm{g} / \mathrm{L}$ & Sample \\
\hline & Cafeteria Creek & 3/1/2007 & $<2$ & $\mu \mathrm{g} / \mathrm{L}$ & Sample \\
\hline & Chicken Creek Downstream & $3 / 1 / 2007$ & $<2$ & $\mu \mathrm{g} / \mathrm{L}$ & Sample \\
\hline & Chicken Creek Upstream & 3/1/2007 & $<2$ & $\mu \mathrm{g} / \mathrm{L}$ & Sample \\
\hline & $\begin{array}{l}\text { N. Fork Strawberry Creek } \\
\text { Downstream }\end{array}$ & 3/1/2007 & $<2$ & $\mu \mathrm{g} / \mathrm{L}$ & Sample \\
\hline & $\begin{array}{l}\text { N. Fork Strawberry Creek } \\
\text { Upstream }\end{array}$ & 3/1/2007 & $<2$ & $\mu \mathrm{g} / \mathrm{L}$ & Sample \\
\hline & No Name Creek & 3/1/2007 & $<2$ & $\mu \mathrm{g} / \mathrm{L}$ & Sample \\
\hline & Ravine Creek & 3/1/2007 & $<2$ & $\mu \mathrm{g} / \mathrm{L}$ & Sample \\
\hline & Ten-Inch Creek & $3 / 1 / 2007$ & $<2$ & $\mu \mathrm{g} / \mathrm{L}$ & Sample \\
\hline \multirow[t]{9}{*}{ Freon 113} & Botanical Garden Creek & $3 / 1 / 2007$ & $<1$ & $\mu \mathrm{g} / \mathrm{L}$ & Sample \\
\hline & Cafeteria Creek & 3/1/2007 & $<1$ & $\mu \mathrm{g} / \mathrm{L}$ & Sample \\
\hline & Chicken Creek Downstream & $3 / 1 / 2007$ & $<1$ & $\mu \mathrm{g} / \mathrm{L}$ & Sample \\
\hline & Chicken Creek Upstream & $3 / 1 / 2007$ & $<1$ & $\mu \mathrm{g} / \mathrm{L}$ & Sample \\
\hline & $\begin{array}{l}\text { N. Fork Strawberry Creek } \\
\text { Downstream }\end{array}$ & 3/1/2007 & $<1$ & $\mu \mathrm{g} / \mathrm{L}$ & Sample \\
\hline & $\begin{array}{l}\text { N. Fork Strawberry Creek } \\
\text { Upstream }\end{array}$ & $3 / 1 / 2007$ & $<1$ & $\mu \mathrm{g} / \mathrm{L}$ & Sample \\
\hline & No Name Creek & 3/1/2007 & $<1$ & $\mu \mathrm{g} / \mathrm{L}$ & Sample \\
\hline & Ravine Creek & 3/1/2007 & $<1$ & $\mu \mathrm{g} / \mathrm{L}$ & Sample \\
\hline & Ten-Inch Creek & 3/1/2007 & $<1$ & $\mu \mathrm{g} / \mathrm{L}$ & Sample \\
\hline \multirow[t]{7}{*}{ Hexachlorobutadiene } & Botanical Garden Creek & $3 / 1 / 2007$ & $<3$ & $\mu \mathrm{g} / \mathrm{L}$ & Sample \\
\hline & Cafeteria Creek & 3/1/2007 & $<3$ & $\mu g / L$ & Sample \\
\hline & Chicken Creek Downstream & 3/1/2007 & $<3$ & $\mu \mathrm{g} / \mathrm{L}$ & Sample \\
\hline & Chicken Creek Upstream & $3 / 1 / 2007$ & $<3$ & $\mu \mathrm{g} / \mathrm{L}$ & Sample \\
\hline & $\begin{array}{l}\text { N. Fork Strawberry Creek } \\
\text { Downstream }\end{array}$ & 3/1/2007 & $<3$ & $\mu \mathrm{g} / \mathrm{L}$ & Sample \\
\hline & $\begin{array}{l}\text { N. Fork Strawberry Creek } \\
\text { Upstream }\end{array}$ & $3 / 1 / 2007$ & $<3$ & $\mu \mathrm{g} / \mathrm{L}$ & Sample \\
\hline & No Name Creek & $3 / 1 / 2007$ & $<3$ & $\mu \mathrm{g} / \mathrm{L}$ & Sample \\
\hline
\end{tabular}




\begin{tabular}{|c|c|c|c|c|c|}
\hline \multicolumn{2}{|c|}{ Volatile Organic Compounds } & \multirow[b]{2}{*}{$\begin{array}{c}\text { Collection } \\
\text { Date }\end{array}$} & \multirow[b]{2}{*}{ Result } & \multirow[b]{2}{*}{ Units } & \multirow[b]{2}{*}{$\begin{array}{c}\text { QA } \\
\text { Type }\end{array}$} \\
\hline Analyte & Location & & & & \\
\hline \multirow[t]{2}{*}{ Hexachlorobutadiene } & Ravine Creek & $3 / 1 / 2007$ & $<3$ & $\mu \mathrm{g} / \mathrm{L}$ & Sample \\
\hline & Ten-Inch Creek & $3 / 1 / 2007$ & $<3$ & $\mu \mathrm{g} / \mathrm{L}$ & Sample \\
\hline \multirow[t]{9}{*}{ Isopropylbenzene } & Botanical Garden Creek & $3 / 1 / 2007$ & $<2$ & $\mu \mathrm{g} / \mathrm{L}$ & Sample \\
\hline & Cafeteria Creek & $3 / 1 / 2007$ & $<2$ & $\mu \mathrm{g} / \mathrm{L}$ & Sample \\
\hline & Chicken Creek Downstream & $3 / 1 / 2007$ & $<2$ & $\mu g / L$ & Sample \\
\hline & Chicken Creek Upstream & $3 / 1 / 2007$ & $<2$ & $\mu \mathrm{g} / \mathrm{L}$ & Sample \\
\hline & $\begin{array}{l}\text { N. Fork Strawberry Creek } \\
\text { Downstream }\end{array}$ & $3 / 1 / 2007$ & $<2$ & $\mu g / L$ & Sample \\
\hline & $\begin{array}{l}\text { N. Fork Strawberry Creek } \\
\text { Upstream }\end{array}$ & $3 / 1 / 2007$ & $<2$ & $\mu \mathrm{g} / \mathrm{L}$ & Sample \\
\hline & No Name Creek & $3 / 1 / 2007$ & $<2$ & $\mu g / L$ & Sample \\
\hline & Ravine Creek & $3 / 1 / 2007$ & $<2$ & $\mu \mathrm{g} / \mathrm{L}$ & Sample \\
\hline & Ten-Inch Creek & $3 / 1 / 2007$ & $<2$ & $\mu \mathrm{g} / \mathrm{L}$ & Sample \\
\hline \multirow[t]{9}{*}{ m- and p-Xylene Isomers } & Botanical Garden Creek & $3 / 1 / 2007$ & $<1$ & $\mu \mathrm{g} / \mathrm{L}$ & Sample \\
\hline & Cafeteria Creek & $3 / 1 / 2007$ & $<1$ & $\mu g / L$ & Sample \\
\hline & Chicken Creek Downstream & $3 / 1 / 2007$ & $<1$ & $\mu \mathrm{g} / \mathrm{L}$ & Sample \\
\hline & Chicken Creek Upstream & $3 / 1 / 2007$ & $<1$ & $\mu \mathrm{g} / \mathrm{L}$ & Sample \\
\hline & $\begin{array}{l}\text { N. Fork Strawberry Creek } \\
\text { Downstream }\end{array}$ & $3 / 1 / 2007$ & $<1$ & $\mu g / L$ & Sample \\
\hline & $\begin{array}{l}\text { N. Fork Strawberry Creek } \\
\text { Upstream }\end{array}$ & $3 / 1 / 2007$ & $<1$ & $\mu g / L$ & Sample \\
\hline & No Name Creek & $3 / 1 / 2007$ & $<1$ & $\mu \mathrm{g} / \mathrm{L}$ & Sample \\
\hline & Ravine Creek & $3 / 1 / 2007$ & $<1$ & $\mu \mathrm{g} / \mathrm{L}$ & Sample \\
\hline & Ten-Inch Creek & $3 / 1 / 2007$ & $<1$ & $\mu \mathrm{g} / \mathrm{L}$ & Sample \\
\hline \multirow[t]{9}{*}{ Methylene chloride } & Botanical Garden Creek & $3 / 1 / 2007$ & $<1$ & $\mu \mathrm{g} / \mathrm{L}$ & Sample \\
\hline & Cafeteria Creek & $3 / 1 / 2007$ & $<1$ & $\mu \mathrm{g} / \mathrm{L}$ & Sample \\
\hline & Chicken Creek Downstream & $3 / 1 / 2007$ & $<1$ & $\mu \mathrm{g} / \mathrm{L}$ & Sample \\
\hline & Chicken Creek Upstream & $3 / 1 / 2007$ & $<1$ & $\mu \mathrm{g} / \mathrm{L}$ & Sample \\
\hline & $\begin{array}{l}\text { N. Fork Strawberry Creek } \\
\text { Downstream }\end{array}$ & $3 / 1 / 2007$ & $<1$ & $\mu g / L$ & Sample \\
\hline & $\begin{array}{l}\text { N. Fork Strawberry Creek } \\
\text { Upstream }\end{array}$ & $3 / 1 / 2007$ & $<1$ & $\mu g / L$ & Sample \\
\hline & No Name Creek & $3 / 1 / 2007$ & $<1$ & $\mu \mathrm{g} / \mathrm{L}$ & Sample \\
\hline & Ravine Creek & $3 / 1 / 2007$ & $<1$ & $\mu \mathrm{g} / \mathrm{L}$ & Sample \\
\hline & Ten-Inch Creek & $3 / 1 / 2007$ & $<1$ & $\mu \mathrm{g} / \mathrm{L}$ & Sample \\
\hline \multirow[t]{3}{*}{ Naphthalene } & Botanical Garden Creek & $3 / 1 / 2007$ & $<2$ & $\mu \mathrm{g} / \mathrm{L}$ & Sample \\
\hline & Cafeteria Creek & $3 / 1 / 2007$ & $<2$ & $\mu \mathrm{g} / \mathrm{L}$ & Sample \\
\hline & Chicken Creek Downstream & $3 / 1 / 2007$ & $<2$ & $\mu \mathrm{g} / \mathrm{L}$ & Sample \\
\hline
\end{tabular}




\begin{tabular}{|c|c|c|c|c|c|}
\hline \multicolumn{2}{|c|}{ Volatile Organic Compounds } & \multirow[b]{2}{*}{$\begin{array}{c}\text { Collection } \\
\text { Date }\end{array}$} & \multirow[b]{2}{*}{ Result } & \multirow[b]{2}{*}{ Units } & \multirow[b]{2}{*}{$\begin{array}{c}\text { QA } \\
\text { Type }\end{array}$} \\
\hline Analyte & Location & & & & \\
\hline \multirow[t]{6}{*}{ Naphthalene } & Chicken Creek Upstream & $3 / 1 / 2007$ & $<2$ & $\mu \mathrm{g} / \mathrm{L}$ & Sample \\
\hline & $\begin{array}{l}\text { N. Fork Strawberry Creek } \\
\text { Downstream }\end{array}$ & $3 / 1 / 2007$ & $<2$ & $\mu \mathrm{g} / \mathrm{L}$ & Sample \\
\hline & $\begin{array}{l}\text { N. Fork Strawberry Creek } \\
\text { Upstream }\end{array}$ & $3 / 1 / 2007$ & $<2$ & $\mu \mathrm{g} / \mathrm{L}$ & Sample \\
\hline & No Name Creek & $3 / 1 / 2007$ & $<2$ & $\mu \mathrm{g} / \mathrm{L}$ & Sample \\
\hline & Ravine Creek & 3/1/2007 & $<2$ & $\mu \mathrm{g} / \mathrm{L}$ & Sample \\
\hline & Ten-Inch Creek & $3 / 1 / 2007$ & $<2$ & $\mu \mathrm{g} / \mathrm{L}$ & Sample \\
\hline \multirow[t]{9}{*}{ n-Butylbenzene } & Botanical Garden Creek & 3/1/2007 & $<1$ & $\mu \mathrm{g} / \mathrm{L}$ & Sample \\
\hline & Cafeteria Creek & $3 / 1 / 2007$ & $<1$ & $\mu \mathrm{g} / \mathrm{L}$ & Sample \\
\hline & Chicken Creek Downstream & $3 / 1 / 2007$ & $<1$ & $\mu \mathrm{g} / \mathrm{L}$ & Sample \\
\hline & Chicken Creek Upstream & 3/1/2007 & $<1$ & $\mu \mathrm{g} / \mathrm{L}$ & Sample \\
\hline & $\begin{array}{l}\text { N. Fork Strawberry Creek } \\
\text { Downstream }\end{array}$ & 3/1/2007 & $<1$ & $\mu \mathrm{g} / \mathrm{L}$ & Sample \\
\hline & $\begin{array}{l}\text { N. Fork Strawberry Creek } \\
\text { Upstream }\end{array}$ & 3/1/2007 & $<1$ & $\mu \mathrm{g} / \mathrm{L}$ & Sample \\
\hline & No Name Creek & 3/1/2007 & $<1$ & $\mu \mathrm{g} / \mathrm{L}$ & Sample \\
\hline & Ravine Creek & $3 / 1 / 2007$ & $<1$ & $\mu \mathrm{g} / \mathrm{L}$ & Sample \\
\hline & Ten-Inch Creek & $3 / 1 / 2007$ & $<1$ & $\mu \mathrm{g} / \mathrm{L}$ & Sample \\
\hline \multirow[t]{9}{*}{ n-Propylbenzene } & Botanical Garden Creek & $3 / 1 / 2007$ & $<1$ & $\mu \mathrm{g} / \mathrm{L}$ & Sample \\
\hline & Cafeteria Creek & 3/1/2007 & $<1$ & $\mu \mathrm{g} / \mathrm{L}$ & Sample \\
\hline & Chicken Creek Downstream & $3 / 1 / 2007$ & $<1$ & $\mu \mathrm{g} / \mathrm{L}$ & Sample \\
\hline & Chicken Creek Upstream & $3 / 1 / 2007$ & $<1$ & $\mu \mathrm{g} / \mathrm{L}$ & Sample \\
\hline & $\begin{array}{l}\text { N. Fork Strawberry Creek } \\
\text { Downstream }\end{array}$ & $3 / 1 / 2007$ & $<1$ & $\mu \mathrm{g} / \mathrm{L}$ & Sample \\
\hline & $\begin{array}{l}\text { N. Fork Strawberry Creek } \\
\text { Upstream }\end{array}$ & $3 / 1 / 2007$ & $<1$ & $\mu \mathrm{g} / \mathrm{L}$ & Sample \\
\hline & No Name Creek & 3/1/2007 & $<1$ & $\mu \mathrm{g} / \mathrm{L}$ & Sample \\
\hline & Ravine Creek & $3 / 1 / 2007$ & $<1$ & $\mu \mathrm{g} / \mathrm{L}$ & Sample \\
\hline & Ten-Inch Creek & $3 / 1 / 2007$ & $<1$ & $\mu \mathrm{g} / \mathrm{L}$ & Sample \\
\hline \multirow[t]{7}{*}{ o-Xylene } & Botanical Garden Creek & $3 / 1 / 2007$ & $<1$ & $\mu \mathrm{g} / \mathrm{L}$ & Sample \\
\hline & Cafeteria Creek & $3 / 1 / 2007$ & $<1$ & $\mu g / L$ & Sample \\
\hline & Chicken Creek Downstream & $3 / 1 / 2007$ & $<1$ & $\mu \mathrm{g} / \mathrm{L}$ & Sample \\
\hline & Chicken Creek Upstream & $3 / 1 / 2007$ & $<1$ & $\mu \mathrm{g} / \mathrm{L}$ & Sample \\
\hline & $\begin{array}{l}\text { N. Fork Strawberry Creek } \\
\text { Downstream }\end{array}$ & 3/1/2007 & $<1$ & $\mu \mathrm{g} / \mathrm{L}$ & Sample \\
\hline & $\begin{array}{l}\text { N. Fork Strawberry Creek } \\
\text { Upstream }\end{array}$ & $3 / 1 / 2007$ & $<1$ & $\mu \mathrm{g} / \mathrm{L}$ & Sample \\
\hline & No Name Creek & 3/1/2007 & $<1$ & $\mu \mathrm{g} / \mathrm{L}$ & Sample \\
\hline
\end{tabular}




\begin{tabular}{|c|c|c|c|c|c|}
\hline \multicolumn{2}{|c|}{ Volatile Organic Compounds } & \multirow[b]{2}{*}{$\begin{array}{c}\text { Collection } \\
\text { Date }\end{array}$} & \multirow[b]{2}{*}{ Result } & \multirow[b]{2}{*}{ Units } & \multirow[b]{2}{*}{$\begin{array}{c}\text { QA } \\
\text { Type }\end{array}$} \\
\hline Analyte & Location & & & & \\
\hline \multirow[t]{2}{*}{0 -Xylene } & Ravine Creek & $3 / 1 / 2007$ & $<1$ & $\mu \mathrm{g} / \mathrm{L}$ & Sample \\
\hline & Ten-Inch Creek & $3 / 1 / 2007$ & $<1$ & $\mu \mathrm{g} / \mathrm{L}$ & Sample \\
\hline \multirow[t]{9}{*}{ p-Isopropyl toluene } & Botanical Garden Creek & $3 / 1 / 2007$ & $<1$ & $\mu g / L$ & Sample \\
\hline & Cafeteria Creek & $3 / 1 / 2007$ & $<1$ & $\mu \mathrm{g} / \mathrm{L}$ & Sample \\
\hline & Chicken Creek Downstream & $3 / 1 / 2007$ & $<1$ & $\mu \mathrm{g} / \mathrm{L}$ & Sample \\
\hline & Chicken Creek Upstream & $3 / 1 / 2007$ & $<1$ & $\mu \mathrm{g} / \mathrm{L}$ & Sample \\
\hline & $\begin{array}{l}\text { N. Fork Strawberry Creek } \\
\text { Downstream }\end{array}$ & $3 / 1 / 2007$ & $<1$ & $\mu g / L$ & Sample \\
\hline & $\begin{array}{l}\text { N. Fork Strawberry Creek } \\
\text { Upstream }\end{array}$ & $3 / 1 / 2007$ & $<1$ & $\mu g / L$ & Sample \\
\hline & No Name Creek & $3 / 1 / 2007$ & $<1$ & $\mu \mathrm{g} / \mathrm{L}$ & Sample \\
\hline & Ravine Creek & $3 / 1 / 2007$ & $<1$ & $\mu \mathrm{g} / \mathrm{L}$ & Sample \\
\hline & Ten-Inch Creek & $3 / 1 / 2007$ & $<1$ & $\mu g / L$ & Sample \\
\hline \multirow[t]{9}{*}{ sec-Butylbenzene } & Botanical Garden Creek & $3 / 1 / 2007$ & $<1$ & $\mu \mathrm{g} / \mathrm{L}$ & Sample \\
\hline & Cafeteria Creek & $3 / 1 / 2007$ & $<1$ & $\mu \mathrm{g} / \mathrm{L}$ & Sample \\
\hline & Chicken Creek Downstream & $3 / 1 / 2007$ & $<1$ & $\mu g / L$ & Sample \\
\hline & Chicken Creek Upstream & $3 / 1 / 2007$ & $<1$ & $\mu \mathrm{g} / \mathrm{L}$ & Sample \\
\hline & $\begin{array}{l}\text { N. Fork Strawberry Creek } \\
\text { Downstream }\end{array}$ & $3 / 1 / 2007$ & $<1$ & $\mu \mathrm{g} / \mathrm{L}$ & Sample \\
\hline & $\begin{array}{l}\text { N. Fork Strawberry Creek } \\
\text { Upstream }\end{array}$ & $3 / 1 / 2007$ & $<1$ & $\mu \mathrm{g} / \mathrm{L}$ & Sample \\
\hline & No Name Creek & $3 / 1 / 2007$ & $<1$ & $\mu \mathrm{g} / \mathrm{L}$ & Sample \\
\hline & Ravine Creek & $3 / 1 / 2007$ & $<1$ & $\mu \mathrm{g} / \mathrm{L}$ & Sample \\
\hline & Ten-Inch Creek & $3 / 1 / 2007$ & $<1$ & $\mu g / L$ & Sample \\
\hline \multirow[t]{9}{*}{ Styrene } & Botanical Garden Creek & $3 / 1 / 2007$ & $<1$ & $\mu g / L$ & Sample \\
\hline & Cafeteria Creek & $3 / 1 / 2007$ & $<1$ & $\mu g / L$ & Sample \\
\hline & Chicken Creek Downstream & $3 / 1 / 2007$ & $<1$ & $\mu \mathrm{g} / \mathrm{L}$ & Sample \\
\hline & Chicken Creek Upstream & $3 / 1 / 2007$ & $<1$ & $\mu \mathrm{g} / \mathrm{L}$ & Sample \\
\hline & $\begin{array}{l}\text { N. Fork Strawberry Creek } \\
\text { Downstream }\end{array}$ & $3 / 1 / 2007$ & $<1$ & $\mu g / L$ & Sample \\
\hline & $\begin{array}{l}\text { N. Fork Strawberry Creek } \\
\text { Upstream }\end{array}$ & $3 / 1 / 2007$ & $<1$ & $\mu g / L$ & Sample \\
\hline & No Name Creek & $3 / 1 / 2007$ & $<1$ & $\mu g / L$ & Sample \\
\hline & Ravine Creek & $3 / 1 / 2007$ & $<1$ & $\mu \mathrm{g} / \mathrm{L}$ & Sample \\
\hline & Ten-Inch Creek & $3 / 1 / 2007$ & $<1$ & $\mu \mathrm{g} / \mathrm{L}$ & Sample \\
\hline \multirow[t]{3}{*}{ tert-Butylbenzene } & Botanical Garden Creek & $3 / 1 / 2007$ & $<1$ & $\mu \mathrm{g} / \mathrm{L}$ & Sample \\
\hline & Cafeteria Creek & $3 / 1 / 2007$ & $<1$ & $\mu \mathrm{g} / \mathrm{L}$ & Sample \\
\hline & Chicken Creek Downstream & $3 / 1 / 2007$ & $<1$ & $\mu \mathrm{g} / \mathrm{L}$ & Sample \\
\hline
\end{tabular}




\begin{tabular}{|c|c|c|c|c|c|}
\hline \multicolumn{2}{|c|}{ Volatile Organic Compounds } & \multirow[b]{2}{*}{$\begin{array}{c}\text { Collection } \\
\text { Date }\end{array}$} & \multirow[b]{2}{*}{ Result } & \multirow[b]{2}{*}{ Units } & \multirow[b]{2}{*}{$\begin{array}{c}\text { QA } \\
\text { Type }\end{array}$} \\
\hline Analyte & Location & & & & \\
\hline \multirow[t]{6}{*}{ tert-Butylbenzene } & Chicken Creek Upstream & $3 / 1 / 2007$ & $<1$ & $\mu \mathrm{g} / \mathrm{L}$ & Sample \\
\hline & $\begin{array}{l}\text { N. Fork Strawberry Creek } \\
\text { Downstream }\end{array}$ & $3 / 1 / 2007$ & $<1$ & $\mu \mathrm{g} / \mathrm{L}$ & Sample \\
\hline & $\begin{array}{l}\text { N. Fork Strawberry Creek } \\
\text { Upstream }\end{array}$ & $3 / 1 / 2007$ & $<1$ & $\mu \mathrm{g} / \mathrm{L}$ & Sample \\
\hline & No Name Creek & $3 / 1 / 2007$ & $<1$ & $\mu \mathrm{g} / \mathrm{L}$ & Sample \\
\hline & Ravine Creek & 3/1/2007 & $<1$ & $\mu \mathrm{g} / \mathrm{L}$ & Sample \\
\hline & Ten-Inch Creek & $3 / 1 / 2007$ & $<1$ & $\mu \mathrm{g} / \mathrm{L}$ & Sample \\
\hline \multirow[t]{9}{*}{ Tetrachloroethene } & Botanical Garden Creek & 3/1/2007 & $<1$ & $\mu \mathrm{g} / \mathrm{L}$ & Sample \\
\hline & Cafeteria Creek & 3/1/2007 & $<1$ & $\mu \mathrm{g} / \mathrm{L}$ & Sample \\
\hline & Chicken Creek Downstream & $3 / 1 / 2007$ & $<1$ & $\mu \mathrm{g} / \mathrm{L}$ & Sample \\
\hline & Chicken Creek Upstream & 3/1/2007 & $<1$ & $\mu \mathrm{g} / \mathrm{L}$ & Sample \\
\hline & $\begin{array}{l}\text { N. Fork Strawberry Creek } \\
\text { Downstream }\end{array}$ & 3/1/2007 & $<1$ & $\mu \mathrm{g} / \mathrm{L}$ & Sample \\
\hline & $\begin{array}{l}\text { N. Fork Strawberry Creek } \\
\text { Upstream }\end{array}$ & 3/1/2007 & $<1$ & $\mu \mathrm{g} / \mathrm{L}$ & Sample \\
\hline & No Name Creek & 3/1/2007 & $<1$ & $\mu \mathrm{g} / \mathrm{L}$ & Sample \\
\hline & Ravine Creek & $3 / 1 / 2007$ & $<1$ & $\mu \mathrm{g} / \mathrm{L}$ & Sample \\
\hline & Ten-Inch Creek & $3 / 1 / 2007$ & $<1$ & $\mu \mathrm{g} / \mathrm{L}$ & Sample \\
\hline \multirow[t]{9}{*}{ Toluene } & Botanical Garden Creek & $3 / 1 / 2007$ & $<1$ & $\mu \mathrm{g} / \mathrm{L}$ & Sample \\
\hline & Cafeteria Creek & 3/1/2007 & $<1$ & $\mu \mathrm{g} / \mathrm{L}$ & Sample \\
\hline & Chicken Creek Downstream & $3 / 1 / 2007$ & $<1$ & $\mu \mathrm{g} / \mathrm{L}$ & Sample \\
\hline & Chicken Creek Upstream & $3 / 1 / 2007$ & $<1$ & $\mu \mathrm{g} / \mathrm{L}$ & Sample \\
\hline & $\begin{array}{l}\text { N. Fork Strawberry Creek } \\
\text { Downstream }\end{array}$ & 3/1/2007 & $<1$ & $\mu \mathrm{g} / \mathrm{L}$ & Sample \\
\hline & $\begin{array}{l}\text { N. Fork Strawberry Creek } \\
\text { Upstream }\end{array}$ & $3 / 1 / 2007$ & $<1$ & $\mu \mathrm{g} / \mathrm{L}$ & Sample \\
\hline & No Name Creek & 3/1/2007 & $<1$ & $\mu \mathrm{g} / \mathrm{L}$ & Sample \\
\hline & Ravine Creek & $3 / 1 / 2007$ & $<1$ & $\mu \mathrm{g} / \mathrm{L}$ & Sample \\
\hline & Ten-Inch Creek & $3 / 1 / 2007$ & $<1$ & $\mu \mathrm{g} / \mathrm{L}$ & Sample \\
\hline \multirow[t]{7}{*}{ trans-1,2-Dichloroethene } & Botanical Garden Creek & $3 / 1 / 2007$ & $<1$ & $\mu \mathrm{g} / \mathrm{L}$ & Sample \\
\hline & Cafeteria Creek & 3/1/2007 & $<1$ & $\mu g / L$ & Sample \\
\hline & Chicken Creek Downstream & 3/1/2007 & $<1$ & $\mu \mathrm{g} / \mathrm{L}$ & Sample \\
\hline & Chicken Creek Upstream & $3 / 1 / 2007$ & $<1$ & $\mu \mathrm{g} / \mathrm{L}$ & Sample \\
\hline & $\begin{array}{l}\text { N. Fork Strawberry Creek } \\
\text { Downstream }\end{array}$ & 3/1/2007 & $<1$ & $\mu \mathrm{g} / \mathrm{L}$ & Sample \\
\hline & $\begin{array}{l}\text { N. Fork Strawberry Creek } \\
\text { Upstream }\end{array}$ & 3/1/2007 & $<1$ & $\mu \mathrm{g} / \mathrm{L}$ & Sample \\
\hline & No Name Creek & $3 / 1 / 2007$ & $<1$ & $\mu \mathrm{g} / \mathrm{L}$ & Sample \\
\hline
\end{tabular}




\begin{tabular}{|c|c|c|c|c|c|}
\hline \multicolumn{2}{|c|}{ Volatile Organic Compounds } & \multirow[b]{2}{*}{$\begin{array}{c}\text { Collection } \\
\text { Date }\end{array}$} & \multirow[b]{2}{*}{ Result } & \multirow[b]{2}{*}{ Units } & \multirow[b]{2}{*}{$\begin{array}{c}\text { QA } \\
\text { Type }\end{array}$} \\
\hline Analyte & Location & & & & \\
\hline \multirow[t]{2}{*}{ trans-1,2-Dichloroethene } & Ravine Creek & $3 / 1 / 2007$ & $<1$ & $\mu \mathrm{g} / \mathrm{L}$ & Sample \\
\hline & Ten-Inch Creek & $3 / 1 / 2007$ & $<1$ & $\mu \mathrm{g} / \mathrm{L}$ & Sample \\
\hline \multirow[t]{9}{*}{ trans-1,3-Dichloropropene } & Botanical Garden Creek & $3 / 1 / 2007$ & $<1$ & $\mu \mathrm{g} / \mathrm{L}$ & Sample \\
\hline & Cafeteria Creek & $3 / 1 / 2007$ & $<1$ & $\mu \mathrm{g} / \mathrm{L}$ & Sample \\
\hline & Chicken Creek Downstream & $3 / 1 / 2007$ & $<1$ & $\mu g / L$ & Sample \\
\hline & Chicken Creek Upstream & $3 / 1 / 2007$ & $<1$ & $\mu \mathrm{g} / \mathrm{L}$ & Sample \\
\hline & $\begin{array}{l}\text { N. Fork Strawberry Creek } \\
\text { Downstream }\end{array}$ & $3 / 1 / 2007$ & $<1$ & $\mu g / L$ & Sample \\
\hline & $\begin{array}{l}\text { N. Fork Strawberry Creek } \\
\text { Upstream }\end{array}$ & $3 / 1 / 2007$ & $<1$ & $\mu \mathrm{g} / \mathrm{L}$ & Sample \\
\hline & No Name Creek & $3 / 1 / 2007$ & $<1$ & $\mu \mathrm{g} / \mathrm{L}$ & Sample \\
\hline & Ravine Creek & $3 / 1 / 2007$ & $<1$ & $\mu \mathrm{g} / \mathrm{L}$ & Sample \\
\hline & Ten-Inch Creek & $3 / 1 / 2007$ & $<1$ & $\mu \mathrm{g} / \mathrm{L}$ & Sample \\
\hline \multirow[t]{9}{*}{ Trichloroethene } & Botanical Garden Creek & $3 / 1 / 2007$ & $<1$ & $\mu \mathrm{g} / \mathrm{L}$ & Sample \\
\hline & Cafeteria Creek & $3 / 1 / 2007$ & $<1$ & $\mu \mathrm{g} / \mathrm{L}$ & Sample \\
\hline & Chicken Creek Downstream & $3 / 1 / 2007$ & $<1$ & $\mu \mathrm{g} / \mathrm{L}$ & Sample \\
\hline & Chicken Creek Upstream & $3 / 1 / 2007$ & $<1$ & $\mu \mathrm{g} / \mathrm{L}$ & Sample \\
\hline & $\begin{array}{l}\text { N. Fork Strawberry Creek } \\
\text { Downstream }\end{array}$ & 3/1/2007 & $<1$ & $\mu \mathrm{g} / \mathrm{L}$ & Sample \\
\hline & $\begin{array}{l}\text { N. Fork Strawberry Creek } \\
\text { Upstream }\end{array}$ & $3 / 1 / 2007$ & $<1$ & $\mu \mathrm{g} / \mathrm{L}$ & Sample \\
\hline & No Name Creek & $3 / 1 / 2007$ & $<1$ & $\mu \mathrm{g} / \mathrm{L}$ & Sample \\
\hline & Ravine Creek & $3 / 1 / 2007$ & $<1$ & $\mu \mathrm{g} / \mathrm{L}$ & Sample \\
\hline & Ten-Inch Creek & $3 / 1 / 2007$ & $<1$ & $\mu \mathrm{g} / \mathrm{L}$ & Sample \\
\hline \multirow[t]{9}{*}{ Vinyl chloride } & Botanical Garden Creek & $3 / 1 / 2007$ & $<1$ & $\mu \mathrm{g} / \mathrm{L}$ & Sample \\
\hline & Cafeteria Creek & $3 / 1 / 2007$ & $<1$ & $\mu \mathrm{g} / \mathrm{L}$ & Sample \\
\hline & Chicken Creek Downstream & $3 / 1 / 2007$ & $<1$ & $\mu \mathrm{g} / \mathrm{L}$ & Sample \\
\hline & Chicken Creek Upstream & $3 / 1 / 2007$ & $<1$ & $\mu \mathrm{g} / \mathrm{L}$ & Sample \\
\hline & $\begin{array}{l}\text { N. Fork Strawberry Creek } \\
\text { Downstream }\end{array}$ & 3/1/2007 & $<1$ & $\mu \mathrm{g} / \mathrm{L}$ & Sample \\
\hline & $\begin{array}{l}\text { N. Fork Strawberry Creek } \\
\text { Upstream }\end{array}$ & $3 / 1 / 2007$ & $<1$ & $\mu \mathrm{g} / \mathrm{L}$ & Sample \\
\hline & No Name Creek & $3 / 1 / 2007$ & $<1$ & $\mu \mathrm{g} / \mathrm{L}$ & Sample \\
\hline & Ravine Creek & $3 / 1 / 2007$ & $<1$ & $\mu \mathrm{g} / \mathrm{L}$ & Sample \\
\hline & Ten-Inch Creek & 3/1/2007 & $<1$ & $\mu \mathrm{g} / \mathrm{L}$ & Sample \\
\hline
\end{tabular}




\begin{tabular}{|c|c|c|c|c|c|c|c|}
\hline \multicolumn{2}{|c|}{ Radiological Activity } & \multirow[b]{2}{*}{$\begin{array}{c}\text { Collection } \\
\text { Date }\end{array}$} & \multicolumn{2}{|c|}{ S.I. } & \multicolumn{2}{|c|}{ Conventional } & \multirow[b]{2}{*}{$\begin{array}{c}\text { QA } \\
\text { Type }\end{array}$} \\
\hline Analyte & Location & & Result & Units & Result & Units & \\
\hline \multirow[t]{11}{*}{ Gross alpha } & $\begin{array}{l}\text { 69-Storm Drain } \\
\text { Manhole }\end{array}$ & 2/7/2007 & $<0.044$ & $\mathrm{~Bq} / \mathrm{L}$ & $<1.2$ & $\mathrm{pCi} / \mathrm{L}$ & Sample \\
\hline & & $10 / 9 / 2007$ & $<0.069$ & $\mathrm{~Bq} / \mathrm{L}$ & $<1.9$ & pCi/L & Sample \\
\hline & Chicken Creek & 2/7/2007 & $<0.069$ & $\mathrm{~Bq} / \mathrm{L}$ & $<1.9$ & pCill & Sample \\
\hline & & $10 / 9 / 2007$ & 0.34 & $\mathrm{~Bq} / \mathrm{L}$ & 9.1 & pCi/L & Sample \\
\hline & & 10/9/2007 & 0.23 & $\mathrm{~Bq} / \mathrm{L}$ & 6.1 & pCi/L & Split \\
\hline & East Canyon & 2/7/2007 & $<0.041$ & $\mathrm{~Bq} / \mathrm{L}$ & $<1.1$ & pCi/L & Sample \\
\hline & & $10 / 9 / 2007$ & 0.16 & $\mathrm{~Bq} / \mathrm{L}$ & 4.4 & pCi/L & Sample \\
\hline & FIELD BLANK & $10 / 10 / 2007$ & $<0.034$ & $\mathrm{~Bq} / \mathrm{L}$ & $<0.91$ & pCi/L & Blank \\
\hline & & $10 / 10 / 2007$ & $<0.054$ & $\mathrm{~Bq} / \mathrm{L}$ & $<1.5$ & pCi/L & Blank \\
\hline & $\begin{array}{l}\text { N. Fork Strawberry } \\
\text { Creek }\end{array}$ & 2/7/2007 & $<0.071$ & $\mathrm{~Bq} / \mathrm{L}$ & $<1.9$ & $\mathrm{pCi} / \mathrm{L}$ & Sample \\
\hline & & $10 / 9 / 2007$ & 0.32 & $\mathrm{~Bq} / \mathrm{L}$ & 8.6 & pCi/L & Sample \\
\hline \multirow[t]{11}{*}{ Gross beta } & $\begin{array}{l}\text { 69-Storm Drain } \\
\text { Manhole }\end{array}$ & 2/7/2007 & 0.11 & $\mathrm{~Bq} / \mathrm{L}$ & 3 & pCi/L & Sample \\
\hline & & $10 / 9 / 2007$ & 0.19 & $\mathrm{~Bq} / \mathrm{L}$ & 5 & pCi/L & Sample \\
\hline & Chicken Creek & 2/7/2007 & $<0.097$ & $\mathrm{~Bq} / \mathrm{L}$ & $<2.6$ & pCi/L & Sample \\
\hline & & 10/9/2007 & 1.2 & $\mathrm{~Bq} / \mathrm{L}$ & 33 & $\mathrm{pCi} / \mathrm{L}$ & Sample \\
\hline & & $10 / 9 / 2007$ & 0.63 & $\mathrm{~Bq} / \mathrm{L}$ & 17 & pCill & Split \\
\hline & East Canyon & 2/7/2007 & 0.19 & $\mathrm{~Bq} / \mathrm{L}$ & 5.1 & $\mathrm{pCi} / \mathrm{L}$ & Sample \\
\hline & & $10 / 9 / 2007$ & 0.56 & $\mathrm{~Bq} / \mathrm{L}$ & 15 & pCi/L & Sample \\
\hline & FIELD BLANK & 10/10/2007 & $<0.081$ & $\mathrm{~Bq} / \mathrm{L}$ & $<2.2$ & pCi/L & Blank \\
\hline & & $10 / 10 / 2007$ & 0.094 & $\mathrm{~Bq} / \mathrm{L}$ & 2.5 & pCi/L & Blank \\
\hline & $\begin{array}{l}\text { N. Fork Strawberry } \\
\text { Creek }\end{array}$ & 2/7/2007 & $<0.098$ & $\mathrm{~Bq} / \mathrm{L}$ & $<2.6$ & pCi/L & Sample \\
\hline & & $10 / 9 / 2007$ & 0.6 & $\mathrm{~Bq} / \mathrm{L}$ & 16 & pCi/L & Sample \\
\hline \multirow[t]{9}{*}{ Tritium } & $\begin{array}{l}\text { 69-Storm Drain } \\
\text { Manhole }\end{array}$ & 2/7/2007 & $<5$ & $\mathrm{~Bq} / \mathrm{L}$ & $<140$ & pCi/L & Sample \\
\hline & & 10/9/2007 & $<5.9$ & $\mathrm{~Bq} / \mathrm{L}$ & $<160$ & $\mathrm{pCi} / \mathrm{L}$ & Sample \\
\hline & Chicken Creek & 2/7/2007 & $<5.3$ & $\mathrm{~Bq} / \mathrm{L}$ & $<140$ & $\mathrm{pCi} / \mathrm{L}$ & Sample \\
\hline & & $10 / 9 / 2007$ & 9.3 & $\mathrm{~Bq} / \mathrm{L}$ & 250 & pCi/L & Sample \\
\hline & & 10/9/2007 & $<10$ & $\mathrm{~Bq} / \mathrm{L}$ & $<270$ & pCill & Split \\
\hline & East Canyon & 2/7/2007 & 5.5 & $\mathrm{~Bq} / \mathrm{L}$ & 150 & pCi/L & Sample \\
\hline & & $10 / 9 / 2007$ & $<6.1$ & $\mathrm{~Bq} / \mathrm{L}$ & $<160$ & $\mathrm{pCi} / \mathrm{L}$ & Sample \\
\hline & FIELD BLANK & $10 / 10 / 2007$ & $<9.6$ & $\mathrm{~Bq} / \mathrm{L}$ & $<260$ & $\mathrm{pCi} / \mathrm{L}$ & Blank \\
\hline & & 10/10/2007 & $<5.9$ & $\mathrm{~Bq} / \mathrm{L}$ & $<160$ & pCi/L & Blank \\
\hline
\end{tabular}




\begin{tabular}{|l|c|c|c|c|c|c|c|}
\hline \multicolumn{2}{|c|}{ Radiological Activity } & & \multicolumn{2}{c|}{ S.I. } & Conventional & \\
\hline Analyte & Location & $\begin{array}{c}\text { Collection } \\
\text { Date }\end{array}$ & Result & Units & Result & Units & $\begin{array}{c}\text { QA } \\
\text { Type }\end{array}$ \\
\hline Tritium & $\begin{array}{l}\text { N. Fork Strawberry } \\
\text { Creek }\end{array}$ & $2 / 7 / 2007$ & $<5.2$ & $\mathrm{~Bq} / \mathrm{L}$ & $<140$ & $\mathrm{pCi} / \mathrm{L}$ & Sample \\
\hline & & $10 / 9 / 2007$ & $<6.1$ & $\mathrm{~Bq} / \mathrm{L}$ & $<160$ & $\mathrm{pC} / \mathrm{L}$ & Sample \\
\hline
\end{tabular}




\begin{tabular}{|c|c|c|c|c|c|}
\hline \multicolumn{2}{|c|}{ General Indicator Parameters } & \multirow[b]{2}{*}{$\begin{array}{l}\text { Collection } \\
\text { Date }\end{array}$} & \multirow[b]{2}{*}{ Result } & \multirow[b]{2}{*}{ Units } & \multirow[b]{2}{*}{$\begin{array}{c}\text { QA } \\
\text { Type }\end{array}$} \\
\hline Analyte & Location & & & & \\
\hline \multirow[t]{8}{*}{ Chemical Oxygen Demand } & $\begin{array}{l}\text { 69-Storm Drain } \\
\text { Manhole }\end{array}$ & 2/7/2007 & 56 & $\mathrm{mg} / \mathrm{L}$ & Sample \\
\hline & & 10/9/2007 & 48 & $\mathrm{mg} / \mathrm{L}$ & Sample \\
\hline & Chicken Creek & 2/7/2007 & $<25$ & $\mathrm{mg} / \mathrm{L}$ & Sample \\
\hline & & 10/9/2007 & 460 & $\mathrm{mg} / \mathrm{L}$ & Sample \\
\hline & & $10 / 9 / 2007$ & 540 & $\mathrm{mg} / \mathrm{L}$ & Split \\
\hline & East Canyon & 2/7/2007 & 39 & $\mathrm{mg} / \mathrm{L}$ & Sample \\
\hline & & 10/9/2007 & 170 & $\mathrm{mg} / \mathrm{L}$ & Sample \\
\hline & $\begin{array}{l}\text { N. Fork Strawberry } \\
\text { Creek }\end{array}$ & 2/7/2007 & $<25$ & $\mathrm{mg} / \mathrm{L}$ & Sample \\
\hline \multirow[t]{8}{*}{ Field pH } & $\begin{array}{l}\text { 69-Storm Drain } \\
\text { Manhole }\end{array}$ & 2/7/2007 & 8.28 & S.U. & Sample \\
\hline & & 10/9/2007 & 7.69 & S.U. & Sample \\
\hline & Chicken Creek & 2/7/2007 & 8.36 & S.U. & Sample \\
\hline & & 10/9/2007 & 7.52 & S.U. & Sample \\
\hline & East Canyon & 2/7/2007 & 8.15 & S.U. & Sample \\
\hline & & 10/9/2007 & 7.71 & S.U. & Sample \\
\hline & $\begin{array}{l}\text { N. Fork Strawberry } \\
\text { Creek }\end{array}$ & 2/7/2007 & 8.65 & S.U. & Sample \\
\hline & & 10/9/2007 & 7.85 & S.U. & Sample \\
\hline \multirow[t]{9}{*}{ Specific Conductance } & $\begin{array}{l}\text { 69-Storm Drain } \\
\text { Manhole }\end{array}$ & 2/7/2007 & 60 & $\mu \mathrm{mhos} / \mathrm{cm}$ & Sample \\
\hline & & 10/9/2007 & 250 & $\mu \mathrm{mhos} / \mathrm{cm}$ & Sample \\
\hline & Chicken Creek & 2/7/2007 & 600 & $\mu \mathrm{mhos} / \mathrm{cm}$ & Sample \\
\hline & & 10/9/2007 & 330 & $\mu \mathrm{mhos} / \mathrm{cm}$ & Sample \\
\hline & & 10/9/2007 & 330 & $\mu \mathrm{mhos} / \mathrm{cm}$ & Split \\
\hline & East Canyon & 2/7/2007 & 71 & $\mu \mathrm{mhos} / \mathrm{cm}$ & Sample \\
\hline & & 10/9/2007 & 88 & $\mu \mathrm{mhos} / \mathrm{cm}$ & Sample \\
\hline & $\begin{array}{l}\text { N. Fork Strawberry } \\
\text { Creek }\end{array}$ & 2/7/2007 & 560 & $\mu \mathrm{mhos} / \mathrm{cm}$ & Sample \\
\hline & & 10/9/2007 & 460 & $\mu \mathrm{mhos} / \mathrm{cm}$ & Sample \\
\hline \multirow[t]{5}{*}{$\begin{array}{l}\text { Total suspended solids } \\
\text { (TSS) }\end{array}$} & $\begin{array}{l}\text { 69-Storm Drain } \\
\text { Manhole }\end{array}$ & 2/7/2007 & 44 & $\mathrm{mg} / \mathrm{L}$ & Sample \\
\hline & & 10/9/2007 & 1.2 & $\mathrm{mg} / \mathrm{L}$ & Sample \\
\hline & Chicken Creek & 2/7/2007 & 7.5 & $\mathrm{mg} / \mathrm{L}$ & Sample \\
\hline & & 10/9/2007 & 390 & $\mathrm{mg} / \mathrm{L}$ & Sample \\
\hline & & $10 / 9 / 2007$ & 390 & $\mathrm{mg} / \mathrm{L}$ & Split \\
\hline
\end{tabular}




\begin{tabular}{|l|l|c|c|c|c|}
\hline \multicolumn{2}{|c|}{ General Indicator Parameters } & & & \\
\hline Analyte & \multicolumn{1}{|c|}{ Location } & $\begin{array}{c}\text { Collection } \\
\text { Date }\end{array}$ & Result & Units & $\begin{array}{c}\text { QA } \\
\text { Type }\end{array}$ \\
\hline $\begin{array}{l}\text { Total suspended solids } \\
\text { (TSS) }\end{array}$ & East Canyon & $2 / 7 / 2007$ & 20 & $\mathrm{mg} / \mathrm{L}$ & Sample \\
\hline & & $10 / 9 / 2007$ & 280 & $\mathrm{mg} / \mathrm{L}$ & Sample \\
\hline & $\begin{array}{l}\text { N. Fork Strawberry } \\
\text { Creek }\end{array}$ & $2 / 7 / 2007$ & 7.5 & $\mathrm{mg} / \mathrm{L}$ & Sample \\
\hline & & $10 / 9 / 2007$ & 310 & $\mathrm{mg} / \mathrm{L}$ & Sample \\
\hline
\end{tabular}




\begin{tabular}{|c|c|c|c|c|c|}
\hline \multicolumn{2}{|r|}{ Metals } & \multirow[b]{2}{*}{$\begin{array}{c}\text { Collection } \\
\text { Date }\end{array}$} & \multirow[b]{2}{*}{ Result } & \multirow[b]{2}{*}{ Units } & \multirow[b]{2}{*}{$\begin{array}{c}\text { QA } \\
\text { Type }\end{array}$} \\
\hline Analyte & Location & & & & \\
\hline \multirow{10}{*}{ Aluminum } & 69-Storm Drain Manhole & 2/7/2007 & 0.74 & $\mathrm{mg} / \mathrm{L}$ & Sample \\
\hline & & $10 / 9 / 2007$ & 0.054 & $\mathrm{mg} / \mathrm{L}$ & Sample \\
\hline & Chicken Creek & 2/7/2007 & 0.22 & $\mathrm{mg} / \mathrm{L}$ & Sample \\
\hline & & 10/9/2007 & 16 & $\mathrm{mg} / \mathrm{L}$ & Sample \\
\hline & & $10 / 9 / 2007$ & 19 & $\mathrm{mg} / \mathrm{L}$ & Split \\
\hline & East Canyon & 2/7/2007 & 0.52 & $\mathrm{mg} / \mathrm{L}$ & Sample \\
\hline & & $10 / 9 / 2007$ & 5.7 & $\mathrm{mg} / \mathrm{L}$ & Sample \\
\hline & FIELD BLANK & $10 / 10 / 2007$ & $<0.05$ & $\mathrm{mg} / \mathrm{L}$ & Blank \\
\hline & N. Fork Strawberry Creek & 2/7/2007 & 0.11 & $\mathrm{mg} / \mathrm{L}$ & Sample \\
\hline & & $10 / 9 / 2007$ & 6.1 & $\mathrm{mg} / \mathrm{L}$ & Sample \\
\hline \multirow[t]{10}{*}{ Iron } & 69-Storm Drain Manhole & 2/7/2007 & 1.1 & $\mathrm{mg} / \mathrm{L}$ & Sample \\
\hline & & 10/9/2007 & 0.066 & $\mathrm{mg} / \mathrm{L}$ & Sample \\
\hline & Chicken Creek & 2/7/2007 & 0.31 & $\mathrm{mg} / \mathrm{L}$ & Sample \\
\hline & & $10 / 9 / 2007$ & 18 & $\mathrm{mg} / \mathrm{L}$ & Sample \\
\hline & & $10 / 9 / 2007$ & 22 & $\mathrm{mg} / \mathrm{L}$ & Split \\
\hline & East Canyon & 2/7/2007 & 0.81 & $\mathrm{mg} / \mathrm{L}$ & Sample \\
\hline & & 10/9/2007 & 8.3 & $\mathrm{mg} / \mathrm{L}$ & Sample \\
\hline & FIELD BLANK & $10 / 10 / 2007$ & $<0.05$ & $\mathrm{mg} / \mathrm{L}$ & Blank \\
\hline & N. Fork Strawberry Creek & 2/7/2007 & 0.23 & $\mathrm{mg} / \mathrm{L}$ & Sample \\
\hline & & 10/9/2007 & 11 & $\mathrm{mg} / \mathrm{L}$ & Sample \\
\hline \multirow[t]{10}{*}{ Magnesium } & 69-Storm Drain Manhole & $2 / 7 / 2007$ & 0.83 & $\mathrm{mg} / \mathrm{L}$ & Sample \\
\hline & & $10 / 9 / 2007$ & 1.6 & $\mathrm{mg} / \mathrm{L}$ & Sample \\
\hline & Chicken Creek & $2 / 7 / 2007$ & 33 & $\mathrm{mg} / \mathrm{L}$ & Sample \\
\hline & & $10 / 9 / 2007$ & 21 & $\mathrm{mg} / \mathrm{L}$ & Sample \\
\hline & & 10/9/2007 & 22 & $\mathrm{mg} / \mathrm{L}$ & Split \\
\hline & East Canyon & $2 / 7 / 2007$ & 1.7 & $\mathrm{mg} / \mathrm{L}$ & Sample \\
\hline & & $10 / 9 / 2007$ & 4.8 & $\mathrm{mg} / \mathrm{L}$ & Sample \\
\hline & FIELD BLANK & $10 / 10 / 2007$ & $<0.05$ & $\mathrm{mg} / \mathrm{L}$ & Blank \\
\hline & N. Fork Strawberry Creek & $2 / 7 / 2007$ & 29 & $\mathrm{mg} / \mathrm{L}$ & Sample \\
\hline & & 10/9/2007 & 24 & $\mathrm{mg} / \mathrm{L}$ & Sample \\
\hline \multirow[t]{4}{*}{ Mercury } & 69-Storm Drain Manhole & $2 / 7 / 2007$ & $<0.0002$ & $\mathrm{mg} / \mathrm{L}$ & Sample \\
\hline & & $10 / 9 / 2007$ & $<0.0002$ & $\mathrm{mg} / \mathrm{L}$ & Sample \\
\hline & Chicken Creek & $2 / 7 / 2007$ & $<0.0002$ & $\mathrm{mg} / \mathrm{L}$ & Sample \\
\hline & & 10/9/2007 & $<0.0002$ & $\mathrm{mg} / \mathrm{L}$ & Sample \\
\hline
\end{tabular}




\begin{tabular}{|c|c|c|c|c|c|}
\hline \multicolumn{2}{|r|}{ Metals } & \multirow[b]{2}{*}{$\begin{array}{l}\text { Collection } \\
\text { Date }\end{array}$} & \multirow[b]{2}{*}{ Result } & \multirow[b]{2}{*}{ Units } & \multirow[b]{2}{*}{$\begin{array}{c}\text { QA } \\
\text { Type }\end{array}$} \\
\hline Analyte & Location & & & & \\
\hline \multirow[t]{6}{*}{ Mercury } & Chicken Creek & $10 / 9 / 2007$ & $<0.0002$ & $\mathrm{mg} / \mathrm{L}$ & Split \\
\hline & East Canyon & 2/7/2007 & $<0.0002$ & $\mathrm{mg} / \mathrm{L}$ & Sample \\
\hline & & $10 / 9 / 2007$ & $<0.0002$ & $\mathrm{mg} / \mathrm{L}$ & Sample \\
\hline & FIELD BLANK & $10 / 10 / 2007$ & $<0.0002$ & $\mathrm{mg} / \mathrm{L}$ & Blank \\
\hline & N. Fork Strawberry Creek & 2/7/2007 & $<0.0002$ & $\mathrm{mg} / \mathrm{L}$ & Sample \\
\hline & & $10 / 9 / 2007$ & 0.00061 & $\mathrm{mg} / \mathrm{L}$ & Sample \\
\hline \multirow[t]{10}{*}{ Zinc } & 69-Storm Drain Manhole & $2 / 7 / 2007$ & 0.085 & $\mathrm{mg} / \mathrm{L}$ & Sample \\
\hline & & $10 / 9 / 2007$ & 0.13 & $\mathrm{mg} / \mathrm{L}$ & Sample \\
\hline & Chicken Creek & $2 / 7 / 2007$ & $<0.05$ & $\mathrm{mg} / \mathrm{L}$ & Sample \\
\hline & & $10 / 9 / 2007$ & 1.2 & $\mathrm{mg} / \mathrm{L}$ & Sample \\
\hline & & $10 / 9 / 2007$ & 1.2 & $\mathrm{mg} / \mathrm{L}$ & Split \\
\hline & East Canyon & $2 / 7 / 2007$ & 0.13 & $\mathrm{mg} / \mathrm{L}$ & Sample \\
\hline & & $10 / 9 / 2007$ & 0.37 & $\mathrm{mg} / \mathrm{L}$ & Sample \\
\hline & FIELD BLANK & $10 / 10 / 2007$ & $<0.05$ & $\mathrm{mg} / \mathrm{L}$ & Blank \\
\hline & N. Fork Strawberry Creek & $2 / 7 / 2007$ & $<0.05$ & $\mathrm{mg} / \mathrm{L}$ & Sample \\
\hline & & $10 / 9 / 2007$ & 0.56 & $\mathrm{mg} / \mathrm{L}$ & Sample \\
\hline
\end{tabular}




\begin{tabular}{|c|c|c|c|c|c|}
\hline \multicolumn{2}{|c|}{ Nutrients } & \multirow[b]{2}{*}{$\begin{array}{c}\text { Collection } \\
\text { Date }\end{array}$} & \multirow[b]{2}{*}{ Result } & \multirow[b]{2}{*}{ Units } & \multirow[b]{2}{*}{$\begin{array}{c}\text { QA } \\
\text { Type }\end{array}$} \\
\hline Analyte & Location & & & & \\
\hline \multirow[t]{9}{*}{ Ammonia Nitrogen (as N) } & $\begin{array}{l}\text { 69-Storm Drain } \\
\text { Manhole }\end{array}$ & 2/7/2007 & 0.34 & $\mathrm{mg} / \mathrm{L}$ & Sample \\
\hline & & $10 / 9 / 2007$ & 0.24 & $\mathrm{mg} / \mathrm{L}$ & Sample \\
\hline & Chicken Creek & 2/7/2007 & $<0.02$ & $\mathrm{mg} / \mathrm{L}$ & Sample \\
\hline & & $10 / 9 / 2007$ & 0.15 & $\mathrm{mg} / \mathrm{L}$ & Sample \\
\hline & & $10 / 9 / 2007$ & 1.1 & $\mathrm{mg} / \mathrm{L}$ & Split \\
\hline & East Canyon & 2/7/2007 & 0.46 & $\mathrm{mg} / \mathrm{L}$ & Sample \\
\hline & & $10 / 9 / 2007$ & 0.27 & $\mathrm{mg} / \mathrm{L}$ & Sample \\
\hline & $\begin{array}{l}\text { N. Fork Strawberry } \\
\text { Creek }\end{array}$ & 2/7/2007 & $<0.02$ & $\mathrm{mg} / \mathrm{L}$ & Sample \\
\hline & & $10 / 9 / 2007$ & 0.84 & $\mathrm{mg} / \mathrm{L}$ & Sample \\
\hline \multirow[t]{5}{*}{ Nitrate plus Nitrite (as N) } & $\begin{array}{l}\text { 69-Storm Drain } \\
\text { Manhole }\end{array}$ & $10 / 9 / 2007$ & 11 & $\mathrm{mg} / \mathrm{L}$ & Sample \\
\hline & Chicken Creek & $10 / 9 / 2007$ & 11 & $\mathrm{mg} / \mathrm{L}$ & Sample \\
\hline & & $10 / 9 / 2007$ & 11 & $\mathrm{mg} / \mathrm{L}$ & Split \\
\hline & East Canyon & $10 / 9 / 2007$ & 2.7 & $\mathrm{mg} / \mathrm{L}$ & Sample \\
\hline & $\begin{array}{l}\text { N. Fork Strawberry } \\
\text { Creek }\end{array}$ & $10 / 9 / 2007$ & 6.7 & $\mathrm{mg} / \mathrm{L}$ & Sample \\
\hline \multirow[t]{4}{*}{$\begin{array}{l}\text { Nitrate plus Nitrite (as } \\
\text { NO3) }\end{array}$} & $\begin{array}{l}\text { 69-Storm Drain } \\
\text { Manhole }\end{array}$ & 2/7/2007 & 2.8 & $\mathrm{mg} / \mathrm{L}$ & Sample \\
\hline & Chicken Creek & 2/7/2007 & 0.77 & $\mathrm{mg} / \mathrm{L}$ & Sample \\
\hline & East Canyon & $2 / 7 / 2007$ & 5 & $\mathrm{mg} / \mathrm{L}$ & Sample \\
\hline & $\begin{array}{l}\text { N. Fork Strawberry } \\
\text { Creek }\end{array}$ & 2/7/2007 & 3.7 & $\mathrm{mg} / \mathrm{L}$ & Sample \\
\hline
\end{tabular}




\begin{tabular}{|c|c|c|c|c|c|}
\hline \multicolumn{2}{|c|}{ Petroleum hydrocarbons } & \multirow[b]{2}{*}{$\begin{array}{c}\text { Collection } \\
\text { Date }\end{array}$} & \multirow[b]{2}{*}{ Result } & \multirow[b]{2}{*}{ Units } & \multirow[b]{2}{*}{$\begin{array}{c}\text { QA } \\
\text { Type }\end{array}$} \\
\hline Analyte & Location & & & & \\
\hline \multirow[t]{10}{*}{$\begin{array}{l}\text { Diesel Range Organics } \\
(\mathrm{C} 12-\mathrm{C} 24)\end{array}$} & $\begin{array}{l}\text { 69-Storm Drain } \\
\text { Manhole }\end{array}$ & 2/7/2007 & 300 & $\mu g / L$ & Sample \\
\hline & & $10 / 9 / 2007$ & 270 & $\mu \mathrm{g} / \mathrm{L}$ & Sample \\
\hline & Chicken Creek & $2 / 7 / 2007$ & 58 & $\mu \mathrm{g} / \mathrm{L}$ & Sample \\
\hline & & $10 / 9 / 2007$ & 1400 & $\mu \mathrm{g} / \mathrm{L}$ & Sample \\
\hline & & $10 / 9 / 2007$ & 1300 & $\mu \mathrm{g} / \mathrm{L}$ & Split \\
\hline & East Canyon & 2/7/2007 & 110 & $\mu \mathrm{g} / \mathrm{L}$ & Sample \\
\hline & & $10 / 9 / 2007$ & 250 & $\mu \mathrm{g} / \mathrm{L}$ & Sample \\
\hline & FIELD BLANK & $10 / 10 / 2007$ & $<50$ & $\mu g / L$ & Blank \\
\hline & $\begin{array}{l}\text { N. Fork Strawberry } \\
\text { Creek }\end{array}$ & 2/7/2007 & 86 & $\mu g / L$ & Sample \\
\hline & & $10 / 9 / 2007$ & 730 & $\mu \mathrm{g} / \mathrm{L}$ & Sample \\
\hline \multirow[t]{9}{*}{ Oil and Grease } & $\begin{array}{l}\text { 69-Storm Drain } \\
\text { Manhole }\end{array}$ & $2 / 7 / 2007$ & $<5$ & $\mathrm{mg} / \mathrm{L}$ & Sample \\
\hline & & $10 / 9 / 2007$ & $<5$ & $\mathrm{mg} / \mathrm{L}$ & Sample \\
\hline & Chicken Creek & 2/7/2007 & $<5$ & $\mathrm{mg} / \mathrm{L}$ & Sample \\
\hline & & $10 / 9 / 2007$ & $<5$ & $\mathrm{mg} / \mathrm{L}$ & Sample \\
\hline & & $10 / 9 / 2007$ & $<5$ & $\mathrm{mg} / \mathrm{L}$ & Split \\
\hline & East Canyon & $2 / 7 / 2007$ & $<5$ & $\mathrm{mg} / \mathrm{L}$ & Sample \\
\hline & & $10 / 9 / 2007$ & $<5$ & $\mathrm{mg} / \mathrm{L}$ & Sample \\
\hline & $\begin{array}{l}\text { N. Fork Strawberry } \\
\text { Creek }\end{array}$ & 2/7/2007 & $<5$ & $\mathrm{mg} / \mathrm{L}$ & Sample \\
\hline & & $10 / 9 / 2007$ & $<5$ & $\mathrm{mg} / \mathrm{L}$ & Sample \\
\hline
\end{tabular}




\begin{tabular}{|c|c|c|c|c|c|c|c|}
\hline \multicolumn{2}{|c|}{ Radiological Activity } & \multirow[b]{2}{*}{$\begin{array}{c}\text { Collection } \\
\text { Date }\end{array}$} & \multicolumn{2}{|c|}{ S.I. } & \multicolumn{2}{|c|}{ Conventional } & \multirow[b]{2}{*}{$\begin{array}{c}\text { QA } \\
\text { Type }\end{array}$} \\
\hline Analyte & Location & & Result & Units & Result & Units & \\
\hline \multirow[t]{32}{*}{ Carbon 14} & FIELD BLANK & $1 / 29 / 2007$ & $<1.3$ & $\mathrm{~Bq} / \mathrm{L}$ & $<34$ & $\mathrm{pCi} / \mathrm{L}$ & Blank \\
\hline & & 1/29/2007 & $<2.7$ & $\mathrm{~Bq} / \mathrm{L}$ & $<74$ & $\mathrm{pCi} / \mathrm{L}$ & Blank \\
\hline & Hearst Sewer & $1 / 3 / 2007$ & $<2.3$ & $\mathrm{~Bq} / \mathrm{L}$ & $<61$ & $\mathrm{pCi} / \mathrm{L}$ & Sample \\
\hline & & $1 / 29 / 2007$ & $<2.8$ & $\mathrm{~Bq} / \mathrm{L}$ & $<75$ & $\mathrm{pCi} / \mathrm{L}$ & Sample \\
\hline & & 2/26/2007 & $<2.4$ & $\mathrm{~Bq} / \mathrm{L}$ & $<65$ & $\mathrm{pCi} / \mathrm{L}$ & Sample \\
\hline & & 3/26/2007 & $<2.7$ & $\mathrm{~Bq} / \mathrm{L}$ & $<74$ & $\mathrm{pCi} / \mathrm{L}$ & Sample \\
\hline & & $4 / 23 / 2007$ & $<2.2$ & $\mathrm{~Bq} / \mathrm{L}$ & $<60$ & $\mathrm{pCi} / \mathrm{L}$ & Sample \\
\hline & & $5 / 23 / 2007$ & $<3.9$ & $\mathrm{~Bq} / \mathrm{L}$ & $<100$ & $\mathrm{pCi} / \mathrm{L}$ & Sample \\
\hline & & $5 / 23 / 2007$ & $<2.3$ & $\mathrm{~Bq} / \mathrm{L}$ & $<62$ & $\mathrm{pCi} / \mathrm{L}$ & Split \\
\hline & & 6/18/2007 & $<2.6$ & $\mathrm{~Bq} / \mathrm{L}$ & $<70$ & $\mathrm{pCi} / \mathrm{L}$ & Sample \\
\hline & & $7 / 16 / 2007$ & $<2.3$ & $\mathrm{~Bq} / \mathrm{L}$ & $<63$ & $\mathrm{pCi} / \mathrm{L}$ & Sample \\
\hline & & 8/13/2007 & $<2.5$ & $\mathrm{~Bq} / \mathrm{L}$ & $<68$ & $\mathrm{pCi} / \mathrm{L}$ & Sample \\
\hline & & 9/10/2007 & $<5.1$ & $\mathrm{~Bq} / \mathrm{L}$ & $<140$ & $\mathrm{pCi} / \mathrm{L}$ & Sample \\
\hline & & $10 / 8 / 2007$ & $<4.6$ & $\mathrm{~Bq} / \mathrm{L}$ & $<120$ & $\mathrm{pCi} / \mathrm{L}$ & Sample \\
\hline & & $11 / 5 / 2007$ & $<2.2$ & $\mathrm{~Bq} / \mathrm{L}$ & $<59$ & $\mathrm{pCi} / \mathrm{L}$ & Sample \\
\hline & & $12 / 3 / 2007$ & $<2.5$ & $\mathrm{~Bq} / \mathrm{L}$ & $<68$ & $\mathrm{pCi} / \mathrm{L}$ & Sample \\
\hline & & $1 / 2 / 2008$ & $<2.3$ & $\mathrm{~Bq} / \mathrm{L}$ & $<63$ & $\mathrm{pCi} / \mathrm{L}$ & Sample \\
\hline & Strawberry Sewer & $1 / 3 / 2007$ & $<2.3$ & $\mathrm{~Bq} / \mathrm{L}$ & $<61$ & $\mathrm{pCi} / \mathrm{L}$ & Sample \\
\hline & & $1 / 29 / 2007$ & $<2.7$ & $\mathrm{~Bq} / \mathrm{L}$ & $<74$ & $\mathrm{pCi} / \mathrm{L}$ & Sample \\
\hline & & $1 / 29 / 2007$ & $<1.3$ & $\mathrm{~Bq} / \mathrm{L}$ & $<34$ & $\mathrm{pCi} / \mathrm{L}$ & Split \\
\hline & & 2/26/2007 & $<2.4$ & $\mathrm{~Bq} / \mathrm{L}$ & $<65$ & $\mathrm{pCi} / \mathrm{L}$ & Sample \\
\hline & & 3/26/2007 & $<2.7$ & $\mathrm{~Bq} / \mathrm{L}$ & $<73$ & $\mathrm{pCi} / \mathrm{L}$ & Sample \\
\hline & & 4/23/2007 & $<2.2$ & $\mathrm{~Bq} / \mathrm{L}$ & $<60$ & $\mathrm{pCi} / \mathrm{L}$ & Sample \\
\hline & & $5 / 23 / 2007$ & $<3.9$ & $\mathrm{~Bq} / \mathrm{L}$ & $<100$ & $\mathrm{pCi} / \mathrm{L}$ & Sample \\
\hline & & $6 / 18 / 2007$ & $<2.5$ & $\mathrm{~Bq} / \mathrm{L}$ & $<69$ & $\mathrm{pCi} / \mathrm{L}$ & Sample \\
\hline & & $7 / 16 / 2007$ & $<2.3$ & $\mathrm{~Bq} / \mathrm{L}$ & $<62$ & $\mathrm{pCi} / \mathrm{L}$ & Sample \\
\hline & & $8 / 13 / 2007$ & $<2.5$ & $\mathrm{~Bq} / \mathrm{L}$ & $<68$ & $\mathrm{pCi} / \mathrm{L}$ & Sample \\
\hline & & 9/10/2007 & $<5$ & $\mathrm{~Bq} / \mathrm{L}$ & $<140$ & $\mathrm{pCi} / \mathrm{L}$ & Sample \\
\hline & & $10 / 8 / 2007$ & $<4.7$ & $\mathrm{~Bq} / \mathrm{L}$ & $<130$ & $\mathrm{pCi} / \mathrm{L}$ & Sample \\
\hline & & $11 / 5 / 2007$ & $<2.2$ & $\mathrm{~Bq} / \mathrm{L}$ & $<58$ & $\mathrm{pCi} / \mathrm{L}$ & Sample \\
\hline & & $12 / 3 / 2007$ & $<2.4$ & $\mathrm{~Bq} / \mathrm{L}$ & $<66$ & $\mathrm{pCi} / \mathrm{L}$ & Sample \\
\hline & & $1 / 2 / 2008$ & $<2.3$ & $\mathrm{~Bq} / \mathrm{L}$ & $<63$ & $\mathrm{pCi} / \mathrm{L}$ & Sample \\
\hline Gross alpha & FIELD BLANK & $1 / 29 / 2007$ & $<0.043$ & $\mathrm{~Bq} / \mathrm{L}$ & $<1.2$ & $\mathrm{pCi} / \mathrm{L}$ & Blank \\
\hline & & $1 / 29 / 2007$ & $<0.037$ & $\mathrm{~Bq} / \mathrm{L}$ & $<1$ & $\mathrm{pCi} / \mathrm{L}$ & Blank \\
\hline
\end{tabular}




\begin{tabular}{|c|c|c|c|c|c|c|c|}
\hline \multicolumn{2}{|c|}{ Radiological Activity } & \multirow[b]{2}{*}{$\begin{array}{c}\text { Collection } \\
\text { Date }\end{array}$} & \multicolumn{2}{|c|}{ S.I. } & \multicolumn{2}{|c|}{ Conventional } & \multirow[b]{2}{*}{$\begin{array}{c}\text { QA } \\
\text { Type }\end{array}$} \\
\hline Analyte & Location & & Result & Units & Result & Units & \\
\hline \multirow[t]{30}{*}{ Gross alpha } & Hearst Sewer & $1 / 3 / 2007$ & $<0.034$ & $\mathrm{~Bq} / \mathrm{L}$ & $<0.91$ & $\mathrm{pCi} / \mathrm{L}$ & Sample \\
\hline & & $1 / 29 / 2007$ & $<0.059$ & $\mathrm{~Bq} / \mathrm{L}$ & $<1.6$ & $\mathrm{pCi} / \mathrm{L}$ & Sample \\
\hline & & $2 / 26 / 2007$ & $<0.063$ & $\mathrm{~Bq} / \mathrm{L}$ & $<1.7$ & $\mathrm{pCi} / \mathrm{L}$ & Sample \\
\hline & & $3 / 26 / 2007$ & $<0.072$ & $\mathrm{~Bq} / \mathrm{L}$ & $<1.9$ & $\mathrm{pCi} / \mathrm{L}$ & Sample \\
\hline & & $4 / 23 / 2007$ & $<0.073$ & $\mathrm{~Bq} / \mathrm{L}$ & $<2$ & $\mathrm{pCi} / \mathrm{L}$ & Sample \\
\hline & & $5 / 23 / 2007$ & $<0.041$ & $\mathrm{~Bq} / \mathrm{L}$ & $<1.1$ & $\mathrm{pCi} / \mathrm{L}$ & Split \\
\hline & & $6 / 18 / 2007$ & $<0.049$ & $\mathrm{~Bq} / \mathrm{L}$ & $<1.3$ & $\mathrm{pCi} / \mathrm{L}$ & Sample \\
\hline & & 7/16/2007 & 0.066 & $\mathrm{~Bq} / \mathrm{L}$ & 1.8 & $\mathrm{pCi} / \mathrm{L}$ & Sample \\
\hline & & $8 / 13 / 2007$ & $<0.054$ & $\mathrm{~Bq} / \mathrm{L}$ & $<1.5$ & $\mathrm{pCi} / \mathrm{L}$ & Sample \\
\hline & & $9 / 10 / 2007$ & $<0.05$ & $\mathrm{~Bq} / \mathrm{L}$ & $<1.3$ & $\mathrm{pCi} / \mathrm{L}$ & Sample \\
\hline & & $10 / 8 / 2007$ & $<0.073$ & $\mathrm{~Bq} / \mathrm{L}$ & $<2$ & $\mathrm{pCi} / \mathrm{L}$ & Sample \\
\hline & & $11 / 5 / 2007$ & $<0.04$ & $\mathrm{~Bq} / \mathrm{L}$ & $<1.1$ & $\mathrm{pCi} / \mathrm{L}$ & Sample \\
\hline & & $12 / 3 / 2007$ & $<0.029$ & $\mathrm{~Bq} / \mathrm{L}$ & $<0.77$ & $\mathrm{pCi} / \mathrm{L}$ & Sample \\
\hline & & $1 / 2 / 2008$ & $<0.07$ & $\mathrm{~Bq} / \mathrm{L}$ & $<1.9$ & $\mathrm{pCi} / \mathrm{L}$ & Sample \\
\hline & Strawberry Sewer & $1 / 3 / 2007$ & $<0.037$ & $\mathrm{~Bq} / \mathrm{L}$ & $<1$ & $\mathrm{pCi} / \mathrm{L}$ & Sample \\
\hline & & $1 / 29 / 2007$ & $<0.055$ & $\mathrm{~Bq} / \mathrm{L}$ & $<1.5$ & $\mathrm{pCi} / \mathrm{L}$ & Sample \\
\hline & & $1 / 29 / 2007$ & $<0.043$ & $\mathrm{~Bq} / \mathrm{L}$ & $<1.2$ & $\mathrm{pCi} / \mathrm{L}$ & Split \\
\hline & & 2/26/2007 & $<0.052$ & $\mathrm{~Bq} / \mathrm{L}$ & $<1.4$ & $\mathrm{pCi} / \mathrm{L}$ & Sample \\
\hline & & $3 / 26 / 2007$ & $<0.049$ & $\mathrm{~Bq} / \mathrm{L}$ & $<1.3$ & $\mathrm{pCi} / \mathrm{L}$ & Sample \\
\hline & & $4 / 23 / 2007$ & $<0.064$ & $\mathrm{~Bq} / \mathrm{L}$ & $<1.7$ & $\mathrm{pCi} / \mathrm{L}$ & Sample \\
\hline & & $5 / 23 / 2007$ & 0.089 & $\mathrm{~Bq} / \mathrm{L}$ & 2.4 & $\mathrm{pCi} / \mathrm{L}$ & Sample \\
\hline & & 6/18/2007 & $<0.064$ & $\mathrm{~Bq} / \mathrm{L}$ & $<1.7$ & $\mathrm{pCi} / \mathrm{L}$ & Sample \\
\hline & & $7 / 16 / 2007$ & 0.063 & $\mathrm{~Bq} / \mathrm{L}$ & 1.7 & $\mathrm{pCi} / \mathrm{L}$ & Sample \\
\hline & & $8 / 13 / 2007$ & $<0.05$ & $\mathrm{~Bq} / \mathrm{L}$ & $<1.4$ & $\mathrm{pCi} / \mathrm{L}$ & Sample \\
\hline & & 9/10/2007 & $<0.074$ & $\mathrm{~Bq} / \mathrm{L}$ & $<2$ & $\mathrm{pCi} / \mathrm{L}$ & Sample \\
\hline & & 9/10/2007 & $<0.045$ & $\mathrm{~Bq} / \mathrm{L}$ & $<1.2$ & $\mathrm{pCi} / \mathrm{L}$ & Split \\
\hline & & $10 / 8 / 2007$ & $<0.044$ & $\mathrm{~Bq} / \mathrm{L}$ & $<1.2$ & $\mathrm{pCi} / \mathrm{L}$ & Sample \\
\hline & & $11 / 5 / 2007$ & $<0.046$ & $\mathrm{~Bq} / \mathrm{L}$ & $<1.2$ & $\mathrm{pCi} / \mathrm{L}$ & Sample \\
\hline & & $12 / 3 / 2007$ & $<0.022$ & $\mathrm{~Bq} / \mathrm{L}$ & $<0.6$ & $\mathrm{pCi} / \mathrm{L}$ & Sample \\
\hline & & $1 / 2 / 2008$ & $<0.069$ & $\mathrm{~Bq} / \mathrm{L}$ & $<1.9$ & $\mathrm{pCi} / \mathrm{L}$ & Sample \\
\hline \multirow[t]{5}{*}{ Gross beta } & FIELD BLANK & $1 / 29 / 2007$ & $<0.076$ & $\mathrm{~Bq} / \mathrm{L}$ & $<2$ & $\mathrm{pCi} / \mathrm{L}$ & Blank \\
\hline & & $1 / 29 / 2007$ & $<0.084$ & $\mathrm{Bg} / \mathrm{L}$ & $<2.3$ & $\mathrm{pCi} / \mathrm{L}$ & Blank \\
\hline & Hearst Sewer & $1 / 3 / 2007$ & 0.45 & $\mathrm{~Bq} / \mathrm{L}$ & 12 & $\mathrm{pCi} / \mathrm{L}$ & Sample \\
\hline & & $1 / 29 / 2007$ & 0.35 & $\mathrm{~Bq} / \mathrm{L}$ & 9.4 & $\mathrm{pCi} / \mathrm{L}$ & Sample \\
\hline & & 2/26/2007 & 0.57 & $\mathrm{~Bq} / \mathrm{L}$ & 15 & $\mathrm{pCi} / \mathrm{L}$ & Sample \\
\hline
\end{tabular}




\begin{tabular}{|c|c|c|c|c|c|c|c|}
\hline \multicolumn{2}{|c|}{ Radiological Activity } & \multirow[b]{2}{*}{$\begin{array}{c}\text { Collection } \\
\text { Date }\end{array}$} & \multicolumn{2}{|c|}{ S.I. } & \multicolumn{2}{|c|}{ Conventional } & \multirow[b]{2}{*}{$\begin{array}{c}\text { QA } \\
\text { Type }\end{array}$} \\
\hline Analyte & Location & & Result & Units & Result & Units & \\
\hline \multirow[t]{28}{*}{ Gross beta } & Hearst Sewer & $3 / 26 / 2007$ & 0.57 & $\mathrm{~Bq} / \mathrm{L}$ & 16 & $\mathrm{pCi} / \mathrm{L}$ & Sample \\
\hline & & 4/23/2007 & 0.59 & $\mathrm{~Bq} / \mathrm{L}$ & 16 & $\mathrm{pCi} / \mathrm{L}$ & Sample \\
\hline & & $5 / 23 / 2007$ & 0.53 & $\mathrm{~Bq} / \mathrm{L}$ & 14 & $\mathrm{pCi} / \mathrm{L}$ & Sample \\
\hline & & $5 / 23 / 2007$ & 0.58 & $\mathrm{~Bq} / \mathrm{L}$ & 16 & $\mathrm{pCi} / \mathrm{L}$ & Split \\
\hline & & $6 / 18 / 2007$ & 0.48 & $\mathrm{~Bq} / \mathrm{L}$ & 13 & $\mathrm{pCi} / \mathrm{L}$ & Sample \\
\hline & & 7/16/2007 & 0.79 & $\mathrm{~Bq} / \mathrm{L}$ & 21 & $\mathrm{pCi} / \mathrm{L}$ & Sample \\
\hline & & $8 / 13 / 2007$ & 0.75 & $\mathrm{~Bq} / \mathrm{L}$ & 20 & $\mathrm{pCi} / \mathrm{L}$ & Sample \\
\hline & & 9/10/2007 & 0.74 & $\mathrm{~Bq} / \mathrm{L}$ & 20 & $\mathrm{pCi} / \mathrm{L}$ & Sample \\
\hline & & $10 / 8 / 2007$ & 0.53 & $\mathrm{~Bq} / \mathrm{L}$ & 14 & $\mathrm{pCi} / \mathrm{L}$ & Sample \\
\hline & & $11 / 5 / 2007$ & 0.47 & $\mathrm{~Bq} / \mathrm{L}$ & 13 & $\mathrm{pCi} / \mathrm{L}$ & Sample \\
\hline & & $12 / 3 / 2007$ & 0.69 & $\mathrm{~Bq} / \mathrm{L}$ & 19 & $\mathrm{pCi} / \mathrm{L}$ & Sample \\
\hline & & $1 / 2 / 2008$ & 0.33 & $\mathrm{~Bq} / \mathrm{L}$ & 8.8 & $\mathrm{pCi} / \mathrm{L}$ & Sample \\
\hline & Strawberry Sewer & $1 / 3 / 2007$ & 0.17 & $\mathrm{~Bq} / \mathrm{L}$ & 4.7 & $\mathrm{pCi} / \mathrm{L}$ & Sample \\
\hline & & $1 / 29 / 2007$ & 0.16 & $\mathrm{~Bq} / \mathrm{L}$ & 4.3 & $\mathrm{pCi} / \mathrm{L}$ & Sample \\
\hline & & $1 / 29 / 2007$ & 0.21 & $\mathrm{~Bq} / \mathrm{L}$ & 5.6 & $\mathrm{pCi} / \mathrm{L}$ & Split \\
\hline & & $2 / 26 / 2007$ & 0.32 & $\mathrm{~Bq} / \mathrm{L}$ & 8.6 & $\mathrm{pCi} / \mathrm{L}$ & Sample \\
\hline & & $3 / 26 / 2007$ & 0.2 & $\mathrm{~Bq} / \mathrm{L}$ & 5.4 & $\mathrm{pCi} / \mathrm{L}$ & Sample \\
\hline & & $4 / 23 / 2007$ & 0.17 & $\mathrm{~Bq} / \mathrm{L}$ & 4.5 & $\mathrm{pCi} / \mathrm{L}$ & Sample \\
\hline & & $5 / 23 / 2007$ & 0.16 & $\mathrm{~Bq} / \mathrm{L}$ & 4.4 & $\mathrm{pCi} / \mathrm{L}$ & Sample \\
\hline & & $6 / 18 / 2007$ & 0.11 & $\mathrm{~Bq} / \mathrm{L}$ & 2.9 & $\mathrm{pCi} / \mathrm{L}$ & Sample \\
\hline & & $7 / 16 / 2007$ & 0.33 & $\mathrm{~Bq} / \mathrm{L}$ & 8.9 & $\mathrm{pCi} / \mathrm{L}$ & Sample \\
\hline & & $8 / 13 / 2007$ & 0.27 & $\mathrm{~Bq} / \mathrm{L}$ & 7.2 & $\mathrm{pCi} / \mathrm{L}$ & Sample \\
\hline & & 9/10/2007 & 0.25 & $\mathrm{~Bq} / \mathrm{L}$ & 6.8 & pCi/L & Sample \\
\hline & & 9/10/2007 & 0.19 & $\mathrm{~Bq} / \mathrm{L}$ & 5.2 & pCi/L & Split \\
\hline & & $10 / 8 / 2007$ & 0.25 & $\mathrm{~Bq} / \mathrm{L}$ & 6.6 & $p C i / L$ & Sample \\
\hline & & $11 / 5 / 2007$ & 0.41 & $\mathrm{~Bq} / \mathrm{L}$ & 11 & pCi/L & Sample \\
\hline & & $12 / 3 / 2007$ & 0.44 & $\mathrm{~Bq} / \mathrm{L}$ & 12 & $\mathrm{pCi} / \mathrm{L}$ & Sample \\
\hline & & $1 / 2 / 2008$ & 0.17 & $\mathrm{~Bq} / \mathrm{L}$ & 4.7 & $\mathrm{pCi} / \mathrm{L}$ & Sample \\
\hline \multirow[t]{7}{*}{ I-125 } & FIELD BLANK & $1 / 29 / 2007$ & $<0.066$ & $\mathrm{~Bq} / \mathrm{L}$ & $<1.8$ & $\mathrm{pCi} / \mathrm{L}$ & Blank \\
\hline & & $1 / 29 / 2007$ & $<0.12$ & $\mathrm{~Bq} / \mathrm{L}$ & $<3.2$ & pCi/L & Blank \\
\hline & Hearst Sewer & $1 / 3 / 2007$ & $<0.13$ & $\mathrm{~Bq} / \mathrm{L}$ & $<3.4$ & $\mathrm{pCi} / \mathrm{L}$ & Sample \\
\hline & & $1 / 29 / 2007$ & $<0.56$ & $\mathrm{~Bq} / \mathrm{L}$ & $<15$ & pCi/L & Sample \\
\hline & & $2 / 26 / 2007$ & $<0.55$ & $\mathrm{~Bq} / \mathrm{L}$ & $<15$ & $\mathrm{pCi} / \mathrm{L}$ & Sample \\
\hline & & $3 / 26 / 2007$ & $<0.21$ & $\mathrm{~Bq} / \mathrm{L}$ & $<5.7$ & $\mathrm{pCi} / \mathrm{L}$ & Sample \\
\hline & & $4 / 23 / 2007$ & $<0.32$ & $\mathrm{~Bq} / \mathrm{L}$ & $<8.7$ & $\mathrm{pCi} / \mathrm{L}$ & Sample \\
\hline
\end{tabular}




\begin{tabular}{|c|c|c|c|c|c|c|c|}
\hline \multicolumn{2}{|c|}{ Radiological Activity } & \multirow[b]{2}{*}{$\begin{array}{c}\text { Collection } \\
\text { Date }\end{array}$} & \multicolumn{2}{|c|}{ S.I. } & \multicolumn{2}{|c|}{ Conventional } & \multirow[b]{2}{*}{$\begin{array}{c}\text { QA } \\
\text { Type }\end{array}$} \\
\hline Analyte & Location & & Result & Units & Result & Units & \\
\hline \multirow[t]{26}{*}{ I-125 } & Hearst Sewer & $5 / 23 / 2007$ & $<0.76$ & $\mathrm{~Bq} / \mathrm{L}$ & $<21$ & $\mathrm{pCi} / \mathrm{L}$ & Sample \\
\hline & & $5 / 23 / 2007$ & $<0.21$ & $\mathrm{~Bq} / \mathrm{L}$ & $<5.7$ & $\mathrm{pCi} / \mathrm{L}$ & Split \\
\hline & & $6 / 18 / 2007$ & $<0.11$ & $\mathrm{~Bq} / \mathrm{L}$ & $<3.1$ & $\mathrm{pCi} / \mathrm{L}$ & Sample \\
\hline & & $7 / 16 / 2007$ & $<0.9$ & $\mathrm{~Bq} / \mathrm{L}$ & $<24$ & $\mathrm{pCi} / \mathrm{L}$ & Sample \\
\hline & & 8/13/2007 & $<0.16$ & $\mathrm{~Bq} / \mathrm{L}$ & $<4.4$ & $\mathrm{pCi} / \mathrm{L}$ & Sample \\
\hline & & 9/10/2007 & $<0.075$ & $\mathrm{~Bq} / \mathrm{L}$ & $<2$ & $\mathrm{pCi} / \mathrm{L}$ & Sample \\
\hline & & $10 / 8 / 2007$ & $<0.19$ & $\mathrm{~Bq} / \mathrm{L}$ & $<5.2$ & $\mathrm{pCi} / \mathrm{L}$ & Sample \\
\hline & & $11 / 5 / 2007$ & $<0.22$ & $\mathrm{~Bq} / \mathrm{L}$ & $<5.9$ & $\mathrm{pCi} / \mathrm{L}$ & Sample \\
\hline & & $12 / 3 / 2007$ & $<0.53$ & $\mathrm{~Bq} / \mathrm{L}$ & $<14$ & $\mathrm{pCi} / \mathrm{L}$ & Sample \\
\hline & & $1 / 2 / 2008$ & $<0.12$ & $\mathrm{~Bq} / \mathrm{L}$ & $<3.1$ & $\mathrm{pCi} / \mathrm{L}$ & Sample \\
\hline & Strawberry Sewer & $1 / 3 / 2007$ & $<0.11$ & $\mathrm{~Bq} / \mathrm{L}$ & $<3$ & $\mathrm{pCi} / \mathrm{L}$ & Sample \\
\hline & & $1 / 29 / 2007$ & $<0.22$ & $\mathrm{~Bq} / \mathrm{L}$ & $<5.9$ & $\mathrm{pCi} / \mathrm{L}$ & Sample \\
\hline & & $1 / 29 / 2007$ & $<0.084$ & $\mathrm{~Bq} / \mathrm{L}$ & $<2.3$ & $\mathrm{pCi} / \mathrm{L}$ & Split \\
\hline & & $2 / 26 / 2007$ & $<0.9$ & $\mathrm{~Bq} / \mathrm{L}$ & $<24$ & $\mathrm{pCi} / \mathrm{L}$ & Sample \\
\hline & & $3 / 26 / 2007$ & $<0.86$ & $\mathrm{~Bq} / \mathrm{L}$ & $<23$ & $\mathrm{pCi} / \mathrm{L}$ & Sample \\
\hline & & $4 / 23 / 2007$ & $<0.18$ & $\mathrm{~Bq} / \mathrm{L}$ & $<5$ & $\mathrm{pCi} / \mathrm{L}$ & Sample \\
\hline & & $5 / 23 / 2007$ & $<0.79$ & $\mathrm{~Bq} / \mathrm{L}$ & $<21$ & $\mathrm{pCi} / \mathrm{L}$ & Sample \\
\hline & & $6 / 18 / 2007$ & $<0.58$ & $\mathrm{~Bq} / \mathrm{L}$ & $<16$ & $\mathrm{pCi} / \mathrm{L}$ & Sample \\
\hline & & $7 / 16 / 2007$ & $<0.85$ & $\mathrm{~Bq} / \mathrm{L}$ & $<23$ & $\mathrm{pCi} / \mathrm{L}$ & Sample \\
\hline & & $8 / 13 / 2007$ & $<0.32$ & $\mathrm{~Bq} / \mathrm{L}$ & $<8.7$ & $\mathrm{pCi} / \mathrm{L}$ & Sample \\
\hline & & 9/10/2007 & $<0.15$ & $\mathrm{~Bq} / \mathrm{L}$ & $<4$ & $\mathrm{pCi} / \mathrm{L}$ & Sample \\
\hline & & 9/10/2007 & $<0.082$ & $\mathrm{~Bq} / \mathrm{L}$ & $<2.2$ & $\mathrm{pCi} / \mathrm{L}$ & Split \\
\hline & & $10 / 8 / 2007$ & $<0.46$ & $\mathrm{~Bq} / \mathrm{L}$ & $<12$ & $\mathrm{pCi} / \mathrm{L}$ & Sample \\
\hline & & $11 / 5 / 2007$ & $<0.24$ & $\mathrm{~Bq} / \mathrm{L}$ & $<6.4$ & $\mathrm{pCi} / \mathrm{L}$ & Sample \\
\hline & & $12 / 3 / 2007$ & $<0.14$ & $\mathrm{~Bq} / \mathrm{L}$ & $<3.9$ & pCi/L & Sample \\
\hline & & $1 / 2 / 2008$ & $<0.4$ & $\mathrm{~Bq} / \mathrm{L}$ & $<11$ & pCi/L & Sample \\
\hline \multirow[t]{9}{*}{ Phosphorus 32} & FIELD BLANK & $1 / 29 / 2007$ & $<0.74$ & $\mathrm{~Bq} / \mathrm{L}$ & $<20$ & $\mathrm{pCi} / \mathrm{L}$ & Blank \\
\hline & Hearst Sewer & $1 / 3 / 2007$ & $<1.6$ & $\mathrm{~Bq} / \mathrm{L}$ & $<43$ & $\mathrm{pCi} / \mathrm{L}$ & Sample \\
\hline & & $1 / 29 / 2007$ & $<1.4$ & $\mathrm{~Bq} / \mathrm{L}$ & $<37$ & $\mathrm{pCi} / \mathrm{L}$ & Sample \\
\hline & & 2/26/2007 & $<1.6$ & $\mathrm{~Bq} / \mathrm{L}$ & $<44$ & $\mathrm{pCi} / \mathrm{L}$ & Sample \\
\hline & & $3 / 26 / 2007$ & $<1.6$ & $\mathrm{~Bq} / \mathrm{L}$ & $<43$ & $\mathrm{pCi} / \mathrm{L}$ & Sample \\
\hline & & $4 / 23 / 2007$ & $<1.7$ & $\mathrm{~Bq} / \mathrm{L}$ & $<47$ & $\mathrm{pCi} / \mathrm{L}$ & Sample \\
\hline & & $5 / 23 / 2007$ & $<1.7$ & $\mathrm{~Bq} / \mathrm{L}$ & $<45$ & $\mathrm{pCi} / \mathrm{L}$ & Sample \\
\hline & & $5 / 23 / 2007$ & $<1.7$ & $\mathrm{~Bq} / \mathrm{L}$ & $<45$ & $\mathrm{pCi} / \mathrm{L}$ & Split \\
\hline & & $6 / 18 / 2007$ & $<1.2$ & $\mathrm{~Bq} / \mathrm{L}$ & $<32$ & $\mathrm{pCi} / \mathrm{L}$ & Sample \\
\hline
\end{tabular}




\begin{tabular}{|c|c|c|c|c|c|c|c|}
\hline \multicolumn{2}{|c|}{ Radiological Activity } & \multirow[b]{2}{*}{$\begin{array}{c}\text { Collection } \\
\text { Date }\end{array}$} & \multicolumn{2}{|c|}{ S.I. } & \multicolumn{2}{|c|}{ Conventional } & \multirow[b]{2}{*}{$\begin{array}{c}\text { QA } \\
\text { Type }\end{array}$} \\
\hline Analyte & Location & & Result & Units & Result & Units & \\
\hline \multirow[t]{23}{*}{ Phosphorus 32} & Hearst Sewer & $7 / 16 / 2007$ & $<1.6$ & $\mathrm{~Bq} / \mathrm{L}$ & $<44$ & $\mathrm{pCi} / \mathrm{L}$ & Sample \\
\hline & & $8 / 13 / 2007$ & $<4.3$ & $\mathrm{~Bq} / \mathrm{L}$ & $<120$ & $\mathrm{pCi} / \mathrm{L}$ & Sample \\
\hline & & 9/10/2007 & $<1.6$ & $\mathrm{~Bq} / \mathrm{L}$ & $<44$ & $\mathrm{pCi} / \mathrm{L}$ & Sample \\
\hline & & $10 / 8 / 2007$ & $<1.5$ & $\mathrm{~Bq} / \mathrm{L}$ & $<41$ & $\mathrm{pCi} / \mathrm{L}$ & Sample \\
\hline & & $11 / 5 / 2007$ & $<1.5$ & $\mathrm{~Bq} / \mathrm{L}$ & $<40$ & $\mathrm{pCi} / \mathrm{L}$ & Sample \\
\hline & & $12 / 3 / 2007$ & $<1.5$ & $\mathrm{~Bq} / \mathrm{L}$ & $<41$ & $\mathrm{pCi} / \mathrm{L}$ & Sample \\
\hline & & $1 / 2 / 2008$ & $<1.6$ & $\mathrm{~Bq} / \mathrm{L}$ & $<44$ & $\mathrm{pCi} / \mathrm{L}$ & Sample \\
\hline & Strawberry Sewer & $1 / 3 / 2007$ & $<1.6$ & $\mathrm{~Bq} / \mathrm{L}$ & $<43$ & $\mathrm{pCi} / \mathrm{L}$ & Sample \\
\hline & & $1 / 29 / 2007$ & $<1.4$ & $\mathrm{~Bq} / \mathrm{L}$ & $<37$ & $\mathrm{pCi} / \mathrm{L}$ & Sample \\
\hline & & $1 / 29 / 2007$ & $<1.4$ & $\mathrm{~Bq} / \mathrm{L}$ & $<37$ & $\mathrm{pCi} / \mathrm{L}$ & Split \\
\hline & & 2/26/2007 & $<1.6$ & $\mathrm{~Bq} / \mathrm{L}$ & $<44$ & $\mathrm{pCi} / \mathrm{L}$ & Sample \\
\hline & & $3 / 26 / 2007$ & $<1.6$ & $\mathrm{~Bq} / \mathrm{L}$ & $<43$ & $\mathrm{pCi} / \mathrm{L}$ & Sample \\
\hline & & 4/23/2007 & $<1.8$ & $\mathrm{~Bq} / \mathrm{L}$ & $<48$ & $\mathrm{pCi} / \mathrm{L}$ & Sample \\
\hline & & $5 / 23 / 2007$ & $<1.7$ & $\mathrm{~Bq} / \mathrm{L}$ & $<45$ & $\mathrm{pCi} / \mathrm{L}$ & Sample \\
\hline & & $6 / 18 / 2007$ & $<1.2$ & $\mathrm{~Bq} / \mathrm{L}$ & $<32$ & $\mathrm{pCi} / \mathrm{L}$ & Sample \\
\hline & & $7 / 16 / 2007$ & $<1.6$ & $\mathrm{~Bq} / \mathrm{L}$ & $<43$ & $\mathrm{pCi} / \mathrm{L}$ & Sample \\
\hline & & $8 / 13 / 2007$ & $<4.4$ & $\mathrm{~Bq} / \mathrm{L}$ & $<120$ & $\mathrm{pCi} / \mathrm{L}$ & Sample \\
\hline & & 9/10/2007 & $<1.8$ & $\mathrm{~Bq} / \mathrm{L}$ & $<50$ & $\mathrm{pCi} / \mathrm{L}$ & Sample \\
\hline & & 9/10/2007 & $<1.7$ & $\mathrm{~Bq} / \mathrm{L}$ & $<47$ & $\mathrm{pCi} / \mathrm{L}$ & Split \\
\hline & & $10 / 8 / 2007$ & $<1.5$ & $\mathrm{~Bq} / \mathrm{L}$ & $<41$ & $\mathrm{pCi} / \mathrm{L}$ & Sample \\
\hline & & $11 / 5 / 2007$ & $<1.5$ & $\mathrm{~Bq} / \mathrm{L}$ & $<40$ & $\mathrm{pCi} / \mathrm{L}$ & Sample \\
\hline & & $12 / 3 / 2007$ & $<1.5$ & $\mathrm{~Bq} / \mathrm{L}$ & $<41$ & pCi/L & Sample \\
\hline & & 1/2/2008 & $<1.7$ & $\mathrm{~Bq} / \mathrm{L}$ & $<45$ & pCi/L & Sample \\
\hline \multirow[t]{12}{*}{ Sulfur 35} & FIELD BLANK & $1 / 29 / 2007$ & $<0.32$ & $\mathrm{~Bq} / \mathrm{L}$ & $<8.7$ & pCi/L & Blank \\
\hline & Hearst Sewer & $1 / 3 / 2007$ & $<0.3$ & $\mathrm{~Bq} / \mathrm{L}$ & $<8.2$ & $p C i / L$ & Sample \\
\hline & & $1 / 29 / 2007$ & $<0.35$ & $\mathrm{~Bq} / \mathrm{L}$ & $<9.4$ & $\mathrm{pCi} / \mathrm{L}$ & Sample \\
\hline & & $2 / 26 / 2007$ & $<0.46$ & $\mathrm{~Bq} / \mathrm{L}$ & $<12$ & $\mathrm{pCi} / \mathrm{L}$ & Sample \\
\hline & & $3 / 26 / 2007$ & $<0.51$ & $\mathrm{~Bq} / \mathrm{L}$ & $<14$ & $\mathrm{pCi} / \mathrm{L}$ & Sample \\
\hline & & 4/23/2007 & $<0.53$ & $\mathrm{~Bq} / \mathrm{L}$ & $<14$ & pCi/L & Sample \\
\hline & & $5 / 23 / 2007$ & $<0.42$ & $\mathrm{~Bq} / \mathrm{L}$ & $<11$ & pCi/L & Sample \\
\hline & & $5 / 23 / 2007$ & $<0.51$ & $\mathrm{~Bq} / \mathrm{L}$ & $<14$ & $p C i / L$ & Split \\
\hline & & 6/18/2007 & $<0.49$ & $\mathrm{~Bq} / \mathrm{L}$ & $<13$ & $\mathrm{pCi} / \mathrm{L}$ & Sample \\
\hline & & 7/16/2007 & $<0.41$ & $\mathrm{~Bq} / \mathrm{L}$ & $<11$ & $p C i / L$ & Sample \\
\hline & & $8 / 13 / 2007$ & $<0.51$ & $\mathrm{~Bq} / \mathrm{L}$ & $<14$ & $\mathrm{pCi} / \mathrm{L}$ & Sample \\
\hline & & 9/10/2007 & $<0.41$ & $\mathrm{~Bq} / \mathrm{L}$ & $<11$ & $\mathrm{pCi} / \mathrm{L}$ & Sample \\
\hline
\end{tabular}




\begin{tabular}{|c|c|c|c|c|c|c|c|}
\hline \multicolumn{2}{|c|}{ Radiological Activity } & \multirow[b]{2}{*}{$\begin{array}{c}\text { Collection } \\
\text { Date }\end{array}$} & \multicolumn{2}{|c|}{ S.I. } & \multicolumn{2}{|c|}{ Conventional } & \multirow[b]{2}{*}{$\begin{array}{c}\text { QA } \\
\text { Type }\end{array}$} \\
\hline Analyte & Location & & Result & Units & Result & Units & \\
\hline \multirow[t]{20}{*}{ Sulfur 35} & Hearst Sewer & $10 / 8 / 2007$ & $<0.34$ & $\mathrm{~Bq} / \mathrm{L}$ & $<9.2$ & $\mathrm{pCi} / \mathrm{L}$ & Sample \\
\hline & & $11 / 5 / 2007$ & $<0.25$ & $\mathrm{~Bq} / \mathrm{L}$ & $<6.6$ & pCi/L & Sample \\
\hline & & $12 / 3 / 2007$ & $<0.43$ & $\mathrm{~Bq} / \mathrm{L}$ & $<12$ & $\mathrm{pCi} / \mathrm{L}$ & Sample \\
\hline & & $1 / 2 / 2008$ & $<0.42$ & $\mathrm{~Bq} / \mathrm{L}$ & $<11$ & $\mathrm{pCi} / \mathrm{L}$ & Sample \\
\hline & Strawberry Sewer & $1 / 3 / 2007$ & $<0.27$ & $\mathrm{~Bq} / \mathrm{L}$ & $<7.2$ & pCi/L & Sample \\
\hline & & $1 / 29 / 2007$ & $<0.42$ & $\mathrm{~Bq} / \mathrm{L}$ & $<11$ & $\mathrm{pCi} / \mathrm{L}$ & Sample \\
\hline & & $1 / 29 / 2007$ & $<0.28$ & $\mathrm{~Bq} / \mathrm{L}$ & $<7.6$ & $\mathrm{pCi} / \mathrm{L}$ & Split \\
\hline & & 2/26/2007 & $<0.46$ & $\mathrm{~Bq} / \mathrm{L}$ & $<12$ & pCi/L & Sample \\
\hline & & $3 / 26 / 2007$ & $<0.49$ & $\mathrm{~Bq} / \mathrm{L}$ & $<13$ & $\mathrm{pCi} / \mathrm{L}$ & Sample \\
\hline & & $4 / 23 / 2007$ & $<0.44$ & $\mathrm{~Bq} / \mathrm{L}$ & $<12$ & $\mathrm{pCi} / \mathrm{L}$ & Sample \\
\hline & & $5 / 23 / 2007$ & $<0.49$ & $\mathrm{~Bq} / \mathrm{L}$ & $<13$ & $\mathrm{pCi} / \mathrm{L}$ & Sample \\
\hline & & $6 / 18 / 2007$ & 0.39 & $\mathrm{~Bq} / \mathrm{L}$ & 11 & $\mathrm{pCi} / \mathrm{L}$ & Sample \\
\hline & & 7/16/2007 & $<0.46$ & $\mathrm{~Bq} / \mathrm{L}$ & $<12$ & pCi/L & Sample \\
\hline & & $8 / 13 / 2007$ & $<0.51$ & $\mathrm{~Bq} / \mathrm{L}$ & $<14$ & pCi/L & Sample \\
\hline & & 9/10/2007 & $<0.4$ & $\mathrm{~Bq} / \mathrm{L}$ & $<11$ & $\mathrm{pCi} / \mathrm{L}$ & Sample \\
\hline & & 9/10/2007 & $<0.4$ & $\mathrm{~Bq} / \mathrm{L}$ & $<11$ & $\mathrm{pCi} / \mathrm{L}$ & Split \\
\hline & & $10 / 8 / 2007$ & $<0.42$ & $\mathrm{~Bq} / \mathrm{L}$ & $<11$ & $\mathrm{pCi} / \mathrm{L}$ & Sample \\
\hline & & $11 / 5 / 2007$ & $<0.25$ & $\mathrm{~Bq} / \mathrm{L}$ & $<6.6$ & $\mathrm{pCi} / \mathrm{L}$ & Sample \\
\hline & & $12 / 3 / 2007$ & $<0.37$ & $\mathrm{~Bq} / \mathrm{L}$ & $<9.9$ & $\mathrm{pCi} / \mathrm{L}$ & Sample \\
\hline & & $1 / 2 / 2008$ & $<0.45$ & $\mathrm{~Bq} / \mathrm{L}$ & $<12$ & pCi/L & Sample \\
\hline \multirow[t]{15}{*}{ Tritium } & FIELD BLANK & $1 / 29 / 2007$ & $<6.5$ & $\mathrm{~Bq} / \mathrm{L}$ & $<180$ & $\mathrm{pCi} / \mathrm{L}$ & Blank \\
\hline & & $1 / 29 / 2007$ & $<10$ & $\mathrm{~Bq} / \mathrm{L}$ & $<280$ & pCi/L & Blank \\
\hline & Hearst Sewer & $1 / 3 / 2007$ & $<7.1$ & $\mathrm{~Bq} / \mathrm{L}$ & $<190$ & $\mathrm{pCi} / \mathrm{L}$ & Sample \\
\hline & & $1 / 29 / 2007$ & $<6.4$ & $\mathrm{~Bq} / \mathrm{L}$ & $<170$ & pCi/L & Sample \\
\hline & & 2/26/2007 & $<5.4$ & $\mathrm{~Bq} / \mathrm{L}$ & $<150$ & pCi/L & Sample \\
\hline & & $3 / 26 / 2007$ & $<6.5$ & $\mathrm{~Bq} / \mathrm{L}$ & $<180$ & pCi/L & Sample \\
\hline & & 4/23/2007 & $<6.4$ & $\mathrm{~Bq} / \mathrm{L}$ & $<170$ & pCi/L & Sample \\
\hline & & $5 / 23 / 2007$ & $<6.4$ & $\mathrm{~Bq} / \mathrm{L}$ & $<170$ & $\mathrm{pCi} / \mathrm{L}$ & Sample \\
\hline & & $5 / 23 / 2007$ & $<7$ & $\mathrm{~Bq} / \mathrm{L}$ & $<190$ & pCi/L & Split \\
\hline & & 6/18/2007 & $<3.7$ & $\mathrm{~Bq} / \mathrm{L}$ & $<99$ & pCi/L & Sample \\
\hline & & 7/16/2007 & $<7.3$ & $\mathrm{~Bq} / \mathrm{L}$ & $<200$ & pCi/L & Sample \\
\hline & & $8 / 13 / 2007$ & $<6$ & $\mathrm{~Bq} / \mathrm{L}$ & $<160$ & pCi/L & Sample \\
\hline & & 9/10/2007 & $<6.2$ & $\mathrm{~Bq} / \mathrm{L}$ & $<170$ & pCi/L & Sample \\
\hline & & $10 / 8 / 2007$ & $<5.8$ & $\mathrm{~Bq} / \mathrm{L}$ & $<160$ & $\mathrm{pCi} / \mathrm{L}$ & Sample \\
\hline & & $11 / 5 / 2007$ & $<5.8$ & $\mathrm{~Bq} / \mathrm{L}$ & $<160$ & pCi/L & Sample \\
\hline
\end{tabular}




\begin{tabular}{|l|c|c|c|c|c|c|c|}
\hline \multicolumn{2}{|c|}{ Radiological Activity } & \multicolumn{2}{c|}{ S.I. } & \multicolumn{2}{c|}{ Conventional } & \\
\hline Analyte & Location & $\begin{array}{c}\text { Collection } \\
\text { Date }\end{array}$ & Result & Units & Result & Units & $\begin{array}{c}\text { QA } \\
\text { Type }\end{array}$ \\
\hline Tritium & Hearst Sewer & $12 / 3 / 2007$ & $<4.9$ & $\mathrm{~Bq} / \mathrm{L}$ & $<130$ & $\mathrm{pCi} / \mathrm{L}$ & Sample \\
\hline & & $1 / 2 / 2008$ & $<6.4$ & $\mathrm{~Bq} / \mathrm{L}$ & $<170$ & $\mathrm{pCi} / \mathrm{L}$ & Sample \\
\hline & Strawberry Sewer & $1 / 3 / 2007$ & $<7.3$ & $\mathrm{~Bq} / \mathrm{L}$ & $<200$ & $\mathrm{pCi} / \mathrm{L}$ & Sample \\
\hline & & $1 / 29 / 2007$ & $<6.4$ & $\mathrm{~Bq} / \mathrm{L}$ & $<170$ & $\mathrm{pCi} / \mathrm{L}$ & Sample \\
\hline & & $1 / 29 / 2007$ & $<10$ & $\mathrm{~Bq} / \mathrm{L}$ & $<280$ & $\mathrm{pCi} / \mathrm{L}$ & Split \\
\hline & & $2 / 26 / 2007$ & $<5.4$ & $\mathrm{~Bq} / \mathrm{L}$ & $<150$ & $\mathrm{pCi} / \mathrm{L}$ & Sample \\
\hline & $3 / 26 / 2007$ & $<6.5$ & $\mathrm{~Bq} / \mathrm{L}$ & $<180$ & $\mathrm{pCi} / \mathrm{L}$ & Sample \\
\hline & & $4 / 23 / 2007$ & $<6.4$ & $\mathrm{~Bq} / \mathrm{L}$ & $<170$ & $\mathrm{pCi} / \mathrm{L}$ & Sample \\
\hline & & $5 / 23 / 2007$ & $<6.5$ & $\mathrm{~Bq} / \mathrm{L}$ & $<180$ & $\mathrm{pCi} / \mathrm{L}$ & Sample \\
\hline & $6 / 18 / 2007$ & $<6$ & $\mathrm{~Bq} / \mathrm{L}$ & $<160$ & $\mathrm{pCi} / \mathrm{L}$ & Sample \\
\hline & & $7 / 16 / 2007$ & $<7.3$ & $\mathrm{~Bq} / \mathrm{L}$ & $<200$ & $\mathrm{pCi} / \mathrm{L}$ & Sample \\
\hline & & $8 / 13 / 2007$ & $<5.9$ & $\mathrm{~Bq} / \mathrm{L}$ & $<160$ & $\mathrm{pCi} / \mathrm{L}$ & Sample \\
\hline & & $9 / 10 / 2007$ & $<6$ & $\mathrm{~Bq} / \mathrm{L}$ & $<160$ & $\mathrm{pCi} / \mathrm{L}$ & Sample \\
\hline & & $9 / 10 / 2007$ & $<7$ & $\mathrm{~Bq} / \mathrm{L}$ & $<190$ & $\mathrm{pCi} / \mathrm{L}$ & Split \\
\hline & & $10 / 8 / 2007$ & $<5.9$ & $\mathrm{~Bq} / \mathrm{L}$ & $<160$ & $\mathrm{pCi} / \mathrm{L}$ & Sample \\
\hline & & $11 / 5 / 2007$ & $<5.9$ & $\mathrm{~Bq} / \mathrm{L}$ & $<160$ & $\mathrm{pCi} / \mathrm{L}$ & Sample \\
\hline & & $12 / 3 / 2007$ & $<4.9$ & $\mathrm{~Bq} / \mathrm{L}$ & $<130$ & $\mathrm{pCi} / \mathrm{L}$ & Sample \\
\hline & & $1 / 2 / 2008$ & $<6.4$ & $\mathrm{~Bq} / \mathrm{L}$ & $<170$ & $\mathrm{pCi} / \mathrm{L}$ & Sample \\
\hline
\end{tabular}




\begin{tabular}{|l|l|c|c|c|c|}
\hline \multicolumn{2}{|c|}{ General Indicator Parameters } & \multicolumn{3}{|c|}{} & \\
\hline Analyte & Location & $\begin{array}{c}\text { Collection } \\
\text { Date }\end{array}$ & Result & Units & $\begin{array}{c}\text { QA } \\
\text { Type }\end{array}$ \\
\hline Chemical Oxygen Demand (Filtered) & Hearst Sewer & $4 / 24 / 2007$ & 140 & $\mathrm{mg} / \mathrm{L}$ & Sample \\
\hline & & $9 / 25 / 2007$ & 75 & $\mathrm{mg} / \mathrm{L}$ & Sample \\
\hline & $\begin{array}{l}\text { Strawberry } \\
\text { Sewer }\end{array}$ & $4 / 24 / 2007$ & 56 & $\mathrm{mg} / \mathrm{L}$ & Sample \\
\hline & & $9 / 25 / 2007$ & $<25$ & $\mathrm{mg} / \mathrm{L}$ & Sample \\
\hline Total suspended solids (TSS) & Hearst Sewer & $4 / 24 / 2007$ & 310 & $\mathrm{mg} / \mathrm{L}$ & Sample \\
\hline & & $9 / 25 / 2007$ & 130 & $\mathrm{mg} / \mathrm{L}$ & Sample \\
\hline & $\begin{array}{l}\text { Strawberry } \\
\text { Sewer }\end{array}$ & $4 / 24 / 2007$ & 200 & $\mathrm{mg} / \mathrm{L}$ & Sample \\
\hline & & $9 / 25 / 2007$ & 83 & $\mathrm{mg} / \mathrm{L}$ & Sample \\
\hline
\end{tabular}




\begin{tabular}{|l|l|c|c|c|c|}
\hline \multicolumn{2}{|c|}{ Metals } & & & \\
\hline Analyte & Location & Collection Date & Result & Units & QA Type \\
\hline Mercury & FIELD BLANK & $9 / 10 / 2007$ & $<0.0002$ & $\mathrm{mg} / \mathrm{L}$ & Blank \\
\hline & Strawberry Sewer & $9 / 10 / 2007$ & $<0.0002$ & $\mathrm{mg} / \mathrm{L}$ & Sample \\
\hline
\end{tabular}




\begin{tabular}{|c|c|c|c|c|c|}
\hline \multicolumn{2}{|c|}{ Volatile Organic Compounds } & \multirow[b]{2}{*}{$\begin{array}{c}\text { Collection } \\
\text { Date }\end{array}$} & \multirow[b]{2}{*}{ Result } & \multirow[b]{2}{*}{ Units } & \multirow[b]{2}{*}{$\begin{array}{c}\text { QA } \\
\text { Type }\end{array}$} \\
\hline Analyte & Location & & & & \\
\hline \multirow[t]{8}{*}{ 1,1,1-Trichloroethane } & FIELD BLANK & $4 / 24 / 2007$ & $<1$ & $\mu \mathrm{g} / \mathrm{L}$ & Blank \\
\hline & & $4 / 24 / 2007$ & $<0.5$ & $\mu \mathrm{g} / \mathrm{L}$ & Blank \\
\hline & Hearst Sewer & $4 / 24 / 2007$ & $<1$ & $\mu \mathrm{g} / \mathrm{L}$ & Sample \\
\hline & & $9 / 24 / 2007$ & $<1$ & $\mu \mathrm{g} / \mathrm{L}$ & Sample \\
\hline & & $9 / 24 / 2007$ & $<0.5$ & $\mu \mathrm{g} / \mathrm{L}$ & Split \\
\hline & Strawberry Sewer & $4 / 24 / 2007$ & $<1$ & $\mu \mathrm{g} / \mathrm{L}$ & Sample \\
\hline & & $4 / 24 / 2007$ & $<0.5$ & $\mu \mathrm{g} / \mathrm{L}$ & Split \\
\hline & & $9 / 24 / 2007$ & $<1$ & $\mu \mathrm{g} / \mathrm{L}$ & Sample \\
\hline \multirow[t]{8}{*}{ 1,1,2,2-Tetrachloroethane } & FIELD BLANK & $4 / 24 / 2007$ & $<1$ & $\mu \mathrm{g} / \mathrm{L}$ & Blank \\
\hline & & $4 / 24 / 2007$ & $<0.5$ & $\mu \mathrm{g} / \mathrm{L}$ & Blank \\
\hline & Hearst Sewer & $4 / 24 / 2007$ & $<1$ & $\mu \mathrm{g} / \mathrm{L}$ & Sample \\
\hline & & 9/24/2007 & $<1$ & $\mu \mathrm{g} / \mathrm{L}$ & Sample \\
\hline & & 9/24/2007 & $<0.5$ & $\mu \mathrm{g} / \mathrm{L}$ & Split \\
\hline & Strawberry Sewer & $4 / 24 / 2007$ & $<1$ & $\mu \mathrm{g} / \mathrm{L}$ & Sample \\
\hline & & $4 / 24 / 2007$ & $<0.5$ & $\mu \mathrm{g} / \mathrm{L}$ & Split \\
\hline & & $9 / 24 / 2007$ & $<1$ & $\mu \mathrm{g} / \mathrm{L}$ & Sample \\
\hline \multirow[t]{8}{*}{ 1,1,2-Trichloroethane } & FIELD BLANK & $4 / 24 / 2007$ & $<0.5$ & $\mu \mathrm{g} / \mathrm{L}$ & Blank \\
\hline & & $4 / 24 / 2007$ & $<1$ & $\mu \mathrm{g} / \mathrm{L}$ & Blank \\
\hline & Hearst Sewer & $4 / 24 / 2007$ & $<1$ & $\mu \mathrm{g} / \mathrm{L}$ & Sample \\
\hline & & 9/24/2007 & $<1$ & $\mu \mathrm{g} / \mathrm{L}$ & Sample \\
\hline & & 9/24/2007 & $<0.5$ & $\mu \mathrm{g} / \mathrm{L}$ & Split \\
\hline & Strawberry Sewer & $4 / 24 / 2007$ & $<1$ & $\mu \mathrm{g} / \mathrm{L}$ & Sample \\
\hline & & $4 / 24 / 2007$ & $<0.5$ & $\mu \mathrm{g} / \mathrm{L}$ & Split \\
\hline & & 9/24/2007 & $<1$ & $\mu \mathrm{g} / \mathrm{L}$ & Sample \\
\hline \multirow[t]{8}{*}{ 1,1-Dichloroethane } & FIELD BLANK & $4 / 24 / 2007$ & $<1$ & $\mu \mathrm{g} / \mathrm{L}$ & Blank \\
\hline & & 4/24/2007 & $<0.5$ & $\mu \mathrm{g} / \mathrm{L}$ & Blank \\
\hline & Hearst Sewer & $4 / 24 / 2007$ & $<1$ & $\mu \mathrm{g} / \mathrm{L}$ & Sample \\
\hline & & 9/24/2007 & $<1$ & $\mu \mathrm{g} / \mathrm{L}$ & Sample \\
\hline & & 9/24/2007 & $<0.5$ & $\mu \mathrm{g} / \mathrm{L}$ & Split \\
\hline & Strawberry Sewer & $4 / 24 / 2007$ & $<1$ & $\mu \mathrm{g} / \mathrm{L}$ & Sample \\
\hline & & $4 / 24 / 2007$ & $<0.5$ & $\mu \mathrm{g} / \mathrm{L}$ & Split \\
\hline & & $9 / 24 / 2007$ & $<1$ & $\mu \mathrm{g} / \mathrm{L}$ & Sample \\
\hline \multirow[t]{2}{*}{ 1,1-Dichloroethene } & FIELD BLANK & 4/24/2007 & $<1$ & $\mu \mathrm{g} / \mathrm{L}$ & Blank \\
\hline & & $4 / 24 / 2007$ & $<0.5$ & $\mu \mathrm{g} / \mathrm{L}$ & Blank \\
\hline
\end{tabular}




\begin{tabular}{|c|c|c|c|c|c|}
\hline \multicolumn{2}{|c|}{ Volatile Organic Compounds } & \multirow[b]{2}{*}{$\begin{array}{c}\text { Collection } \\
\text { Date }\end{array}$} & \multirow[b]{2}{*}{ Result } & \multirow[b]{2}{*}{ Units } & \multirow[b]{2}{*}{$\begin{array}{c}\text { QA } \\
\text { Type }\end{array}$} \\
\hline Analyte & Location & & & & \\
\hline \multirow[t]{6}{*}{ 1,1-Dichloroethene } & Hearst Sewer & 4/24/2007 & $<1$ & $\mu \mathrm{g} / \mathrm{L}$ & Sample \\
\hline & & 9/24/2007 & $<1$ & $\mu \mathrm{g} / \mathrm{L}$ & Sample \\
\hline & & 9/24/2007 & $<0.5$ & $\mu \mathrm{g} / \mathrm{L}$ & Split \\
\hline & Strawberry Sewer & 4/24/2007 & $<1$ & $\mu \mathrm{g} / \mathrm{L}$ & Sample \\
\hline & & 4/24/2007 & $<0.5$ & $\mu \mathrm{g} / \mathrm{L}$ & Split \\
\hline & & 9/24/2007 & $<1$ & $\mu \mathrm{g} / \mathrm{L}$ & Sample \\
\hline \multirow[t]{8}{*}{ 1,2-Dichlorobenzene } & FIELD BLANK & 4/24/2007 & $<0.5$ & $\mu \mathrm{g} / \mathrm{L}$ & Blank \\
\hline & & 4/24/2007 & $<1$ & $\mu \mathrm{g} / \mathrm{L}$ & Blank \\
\hline & Hearst Sewer & $4 / 24 / 2007$ & $<1$ & $\mu \mathrm{g} / \mathrm{L}$ & Sample \\
\hline & & 9/24/2007 & $<1$ & $\mu \mathrm{g} / \mathrm{L}$ & Sample \\
\hline & & 9/24/2007 & $<0.5$ & $\mu \mathrm{g} / \mathrm{L}$ & Split \\
\hline & Strawberry Sewer & 4/24/2007 & $<1$ & $\mu \mathrm{g} / \mathrm{L}$ & Sample \\
\hline & & 4/24/2007 & $<0.5$ & $\mu \mathrm{g} / \mathrm{L}$ & Split \\
\hline & & 9/24/2007 & $<1$ & $\mu \mathrm{g} / \mathrm{L}$ & Sample \\
\hline \multirow[t]{8}{*}{ 1,2-Dichloroethane } & FIELD BLANK & $4 / 24 / 2007$ & $<0.5$ & $\mu \mathrm{g} / \mathrm{L}$ & Blank \\
\hline & & 4/24/2007 & $<1$ & $\mu \mathrm{g} / \mathrm{L}$ & Blank \\
\hline & Hearst Sewer & $4 / 24 / 2007$ & $<1$ & $\mu \mathrm{g} / \mathrm{L}$ & Sample \\
\hline & & 9/24/2007 & $<1$ & $\mu \mathrm{g} / \mathrm{L}$ & Sample \\
\hline & & 9/24/2007 & $<0.5$ & $\mu \mathrm{g} / \mathrm{L}$ & Split \\
\hline & Strawberry Sewer & $4 / 24 / 2007$ & $<1$ & $\mu \mathrm{g} / \mathrm{L}$ & Sample \\
\hline & & 4/24/2007 & $<0.5$ & $\mu \mathrm{g} / \mathrm{L}$ & Split \\
\hline & & 9/24/2007 & $<1$ & $\mu \mathrm{g} / \mathrm{L}$ & Sample \\
\hline \multirow[t]{6}{*}{ 1,2-Dichloroethene (total) } & FIELD BLANK & $4 / 24 / 2007$ & $<1$ & $\mu g / L$ & Blank \\
\hline & Hearst Sewer & $4 / 24 / 2007$ & $<1$ & $\mu \mathrm{g} / \mathrm{L}$ & Sample \\
\hline & & 9/24/2007 & $<1$ & $\mu \mathrm{g} / \mathrm{L}$ & Sample \\
\hline & & 9/24/2007 & $<0.5$ & $\mu g / L$ & Split \\
\hline & Strawberry Sewer & $4 / 24 / 2007$ & $<1$ & $\mu g / L$ & Sample \\
\hline & & 9/24/2007 & $<1$ & $\mu \mathrm{g} / \mathrm{L}$ & Sample \\
\hline \multirow[t]{7}{*}{ 1,2-Dichloropropane } & FIELD BLANK & 4/24/2007 & $<0.5$ & $\mu \mathrm{g} / \mathrm{L}$ & Blank \\
\hline & & 4/24/2007 & $<1$ & $\mu \mathrm{g} / \mathrm{L}$ & Blank \\
\hline & Hearst Sewer & $4 / 24 / 2007$ & $<1$ & $\mu \mathrm{g} / \mathrm{L}$ & Sample \\
\hline & & 9/24/2007 & $<1$ & $\mu \mathrm{g} / \mathrm{L}$ & Sample \\
\hline & & 9/24/2007 & $<0.5$ & $\mu \mathrm{g} / \mathrm{L}$ & Split \\
\hline & Strawberry Sewer & $4 / 24 / 2007$ & $<1$ & $\mu \mathrm{g} / \mathrm{L}$ & Sample \\
\hline & & $4 / 24 / 2007$ & $<0.5$ & $\mu \mathrm{g} / \mathrm{L}$ & Split \\
\hline
\end{tabular}




\begin{tabular}{|c|c|c|c|c|c|}
\hline \multicolumn{2}{|c|}{ Volatile Organic Compounds } & \multirow[b]{2}{*}{$\begin{array}{c}\text { Collection } \\
\text { Date }\end{array}$} & \multirow[b]{2}{*}{ Result } & \multirow[b]{2}{*}{ Units } & \multirow[b]{2}{*}{$\begin{array}{c}\text { QA } \\
\text { Type }\end{array}$} \\
\hline Analyte & Location & & & & \\
\hline 1,2-Dichloropropane & Strawberry Sewer & 9/24/2007 & $<1$ & $\mu \mathrm{g} / \mathrm{L}$ & Sample \\
\hline \multirow[t]{8}{*}{ 1,3-Dichlorobenzene } & FIELD BLANK & 4/24/2007 & $<0.5$ & $\mu \mathrm{g} / \mathrm{L}$ & Blank \\
\hline & & $4 / 24 / 2007$ & $<1$ & $\mu \mathrm{g} / \mathrm{L}$ & Blank \\
\hline & Hearst Sewer & 4/24/2007 & $<1$ & $\mu \mathrm{g} / \mathrm{L}$ & Sample \\
\hline & & 9/24/2007 & $<1$ & $\mu \mathrm{g} / \mathrm{L}$ & Sample \\
\hline & & 9/24/2007 & $<0.5$ & $\mu \mathrm{g} / \mathrm{L}$ & Split \\
\hline & Strawberry Sewer & 4/24/2007 & $<1$ & $\mu \mathrm{g} / \mathrm{L}$ & Sample \\
\hline & & 4/24/2007 & $<0.5$ & $\mu \mathrm{g} / \mathrm{L}$ & Split \\
\hline & & 9/24/2007 & $<1$ & $\mu \mathrm{g} / \mathrm{L}$ & Sample \\
\hline \multirow[t]{8}{*}{ 1,4-Dichlorobenzene } & FIELD BLANK & 4/24/2007 & $<1$ & $\mu \mathrm{g} / \mathrm{L}$ & Blank \\
\hline & & 4/24/2007 & $<0.5$ & $\mu \mathrm{g} / \mathrm{L}$ & Blank \\
\hline & Hearst Sewer & 4/24/2007 & $<1$ & $\mu g / L$ & Sample \\
\hline & & 9/24/2007 & $<1$ & $\mu \mathrm{g} / \mathrm{L}$ & Sample \\
\hline & & 9/24/2007 & $<0.5$ & $\mu \mathrm{g} / \mathrm{L}$ & Split \\
\hline & Strawberry Sewer & $4 / 24 / 2007$ & $<1$ & $\mu \mathrm{g} / \mathrm{L}$ & Sample \\
\hline & & $4 / 24 / 2007$ & $<0.5$ & $\mu \mathrm{g} / \mathrm{L}$ & Split \\
\hline & & 9/24/2007 & $<1$ & $\mu \mathrm{g} / \mathrm{L}$ & Sample \\
\hline \multirow[t]{6}{*}{ 2-Butanone } & FIELD BLANK & $4 / 24 / 2007$ & $<20$ & $\mu g / L$ & Blank \\
\hline & Hearst Sewer & 4/24/2007 & $<20$ & $\mu \mathrm{g} / \mathrm{L}$ & Sample \\
\hline & & 9/24/2007 & $<20$ & $\mu g / L$ & Sample \\
\hline & & 9/24/2007 & $<5$ & $\mu \mathrm{g} / \mathrm{L}$ & Split \\
\hline & Strawberry Sewer & 4/24/2007 & $<20$ & $\mu \mathrm{g} / \mathrm{L}$ & Sample \\
\hline & & 9/24/2007 & $<20$ & $\mu g / L$ & Sample \\
\hline \multirow[t]{6}{*}{ 2-Chloroethylvinylether } & FIELD BLANK & 4/24/2007 & $<20$ & $\mu g / L$ & Blank \\
\hline & Hearst Sewer & 4/24/2007 & $<20$ & $\mu \mathrm{g} / \mathrm{L}$ & Sample \\
\hline & & 9/24/2007 & $<20$ & $\mu g / L$ & Sample \\
\hline & & 9/24/2007 & $<10$ & $\mu g / L$ & Split \\
\hline & Strawberry Sewer & $4 / 24 / 2007$ & $<20$ & $\mu g / L$ & Sample \\
\hline & & 9/24/2007 & $<20$ & $\mu \mathrm{g} / \mathrm{L}$ & Sample \\
\hline \multirow[t]{6}{*}{ 2-Hexanone } & FIELD BLANK & 4/24/2007 & $<20$ & $\mu g / L$ & Blank \\
\hline & Hearst Sewer & $4 / 24 / 2007$ & $<20$ & $\mu g / L$ & Sample \\
\hline & & 9/24/2007 & $<20$ & $\mu \mathrm{g} / \mathrm{L}$ & Sample \\
\hline & & 9/24/2007 & $<5$ & $\mu \mathrm{g} / \mathrm{L}$ & Split \\
\hline & Strawberry Sewer & $4 / 24 / 2007$ & $<20$ & $\mu \mathrm{g} / \mathrm{L}$ & Sample \\
\hline & & 9/24/2007 & $<20$ & $\mu g / L$ & Sample \\
\hline
\end{tabular}




\begin{tabular}{|c|c|c|c|c|c|}
\hline \multicolumn{2}{|c|}{ Volatile Organic Compounds } & \multirow[b]{2}{*}{$\begin{array}{c}\text { Collection } \\
\text { Date }\end{array}$} & \multirow[b]{2}{*}{ Result } & \multirow[b]{2}{*}{ Units } & \multirow[b]{2}{*}{$\begin{array}{c}\text { QA } \\
\text { Type }\end{array}$} \\
\hline Analyte & Location & & & & \\
\hline \multirow[t]{6}{*}{ 4-Methyl-2-pentanone } & FIELD BLANK & 4/24/2007 & $<20$ & $\mu \mathrm{g} / \mathrm{L}$ & Blank \\
\hline & Hearst Sewer & $4 / 24 / 2007$ & $<20$ & $\mu \mathrm{g} / \mathrm{L}$ & Sample \\
\hline & & 9/24/2007 & $<20$ & $\mu \mathrm{g} / \mathrm{L}$ & Sample \\
\hline & & 9/24/2007 & $<5$ & $\mu \mathrm{g} / \mathrm{L}$ & Split \\
\hline & Strawberry Sewer & 4/24/2007 & $<20$ & $\mu \mathrm{g} / \mathrm{L}$ & Sample \\
\hline & & 9/24/2007 & $<20$ & $\mu \mathrm{g} / \mathrm{L}$ & Sample \\
\hline \multirow[t]{6}{*}{ Acetone } & FIELD BLANK & 4/24/2007 & $<10$ & $\mu \mathrm{g} / \mathrm{L}$ & Blank \\
\hline & Hearst Sewer & 4/24/2007 & 36 & $\mu \mathrm{g} / \mathrm{L}$ & Sample \\
\hline & & 9/24/2007 & 13 & $\mu g / L$ & Sample \\
\hline & & 9/24/2007 & 20 & $\mu \mathrm{g} / \mathrm{L}$ & Split \\
\hline & Strawberry Sewer & 4/24/2007 & 2100 & $\mu \mathrm{g} / \mathrm{L}$ & Sample \\
\hline & & 9/24/2007 & 35 & $\mu \mathrm{g} / \mathrm{L}$ & Sample \\
\hline \multirow[t]{8}{*}{ Benzene } & FIELD BLANK & 4/24/2007 & $<0.5$ & $\mu \mathrm{g} / \mathrm{L}$ & Blank \\
\hline & & 4/24/2007 & $<1$ & $\mu \mathrm{g} / \mathrm{L}$ & Blank \\
\hline & Hearst Sewer & $4 / 24 / 2007$ & $<1$ & $\mu \mathrm{g} / \mathrm{L}$ & Sample \\
\hline & & 9/24/2007 & $<1$ & $\mu \mathrm{g} / \mathrm{L}$ & Sample \\
\hline & & 9/24/2007 & $<0.5$ & $\mu \mathrm{g} / \mathrm{L}$ & Split \\
\hline & Strawberry Sewer & $4 / 24 / 2007$ & $<1$ & $\mu \mathrm{g} / \mathrm{L}$ & Sample \\
\hline & & 4/24/2007 & $<0.5$ & $\mu \mathrm{g} / \mathrm{L}$ & Split \\
\hline & & 9/24/2007 & $<1$ & $\mu \mathrm{g} / \mathrm{L}$ & Sample \\
\hline \multirow[t]{8}{*}{ Bromodichloromethane } & FIELD BLANK & 4/24/2007 & $<1$ & $\mu \mathrm{g} / \mathrm{L}$ & Blank \\
\hline & & 4/24/2007 & $<0.5$ & $\mu \mathrm{g} / \mathrm{L}$ & Blank \\
\hline & Hearst Sewer & $4 / 24 / 2007$ & $<1$ & $\mu g / L$ & Sample \\
\hline & & 9/24/2007 & $<1$ & $\mu \mathrm{g} / \mathrm{L}$ & Sample \\
\hline & & 9/24/2007 & 0.6 & $\mu \mathrm{g} / \mathrm{L}$ & Split \\
\hline & Strawberry Sewer & $4 / 24 / 2007$ & $<1$ & $\mu g / L$ & Sample \\
\hline & & $4 / 24 / 2007$ & $<0.5$ & $\mu g / L$ & Split \\
\hline & & 9/24/2007 & $<1$ & $\mu \mathrm{g} / \mathrm{L}$ & Sample \\
\hline \multirow[t]{7}{*}{ Bromoform } & FIELD BLANK & 4/24/2007 & $<0.5$ & $\mu \mathrm{g} / \mathrm{L}$ & Blank \\
\hline & & $4 / 24 / 2007$ & $<1$ & $\mu \mathrm{g} / \mathrm{L}$ & Blank \\
\hline & Hearst Sewer & $4 / 24 / 2007$ & $<1$ & $\mu \mathrm{g} / \mathrm{L}$ & Sample \\
\hline & & 9/24/2007 & $<1$ & $\mu g / L$ & Sample \\
\hline & & 9/24/2007 & $<0.5$ & $\mu \mathrm{g} / \mathrm{L}$ & Split \\
\hline & Strawberry Sewer & $4 / 24 / 2007$ & $<1$ & $\mu \mathrm{g} / \mathrm{L}$ & Sample \\
\hline & & $4 / 24 / 2007$ & $<0.5$ & $\mu \mathrm{g} / \mathrm{L}$ & Split \\
\hline
\end{tabular}




\begin{tabular}{|c|c|c|c|c|c|}
\hline \multicolumn{2}{|c|}{ Volatile Organic Compounds } & \multirow[b]{2}{*}{$\begin{array}{c}\text { Collection } \\
\text { Date }\end{array}$} & \multirow[b]{2}{*}{ Result } & \multirow[b]{2}{*}{ Units } & \multirow[b]{2}{*}{$\begin{array}{c}\text { QA } \\
\text { Type }\end{array}$} \\
\hline Analyte & Location & & & & \\
\hline Bromoform & Strawberry Sewer & 9/24/2007 & $<1$ & $\mu \mathrm{g} / \mathrm{L}$ & Sample \\
\hline \multirow[t]{8}{*}{ Bromomethane } & FIELD BLANK & $4 / 24 / 2007$ & $<1$ & $\mu \mathrm{g} / \mathrm{L}$ & Blank \\
\hline & & $4 / 24 / 2007$ & $<2$ & $\mu \mathrm{g} / \mathrm{L}$ & Blank \\
\hline & Hearst Sewer & $4 / 24 / 2007$ & $<2$ & $\mu \mathrm{g} / \mathrm{L}$ & Sample \\
\hline & & 9/24/2007 & $<2$ & $\mu \mathrm{g} / \mathrm{L}$ & Sample \\
\hline & & 9/24/2007 & $<0.5$ & $\mu \mathrm{g} / \mathrm{L}$ & Split \\
\hline & Strawberry Sewer & $4 / 24 / 2007$ & $<2$ & $\mu \mathrm{g} / \mathrm{L}$ & Sample \\
\hline & & $4 / 24 / 2007$ & $<1$ & $\mu \mathrm{g} / \mathrm{L}$ & Split \\
\hline & & 9/24/2007 & $<2$ & $\mu \mathrm{g} / \mathrm{L}$ & Sample \\
\hline \multirow[t]{6}{*}{ Carbon disulfide } & FIELD BLANK & $4 / 24 / 2007$ & $<1$ & $\mu \mathrm{g} / \mathrm{L}$ & Blank \\
\hline & Hearst Sewer & $4 / 24 / 2007$ & $<1$ & $\mu \mathrm{g} / \mathrm{L}$ & Sample \\
\hline & & 9/24/2007 & $<1$ & $\mu \mathrm{g} / \mathrm{L}$ & Sample \\
\hline & & 9/24/2007 & $<1$ & $\mu \mathrm{g} / \mathrm{L}$ & Split \\
\hline & Strawberry Sewer & $4 / 24 / 2007$ & $<1$ & $\mu \mathrm{g} / \mathrm{L}$ & Sample \\
\hline & & 9/24/2007 & $<1$ & $\mu \mathrm{g} / \mathrm{L}$ & Sample \\
\hline \multirow[t]{8}{*}{ Carbon tetrachloride } & FIELD BLANK & $4 / 24 / 2007$ & $<1$ & $\mu \mathrm{g} / \mathrm{L}$ & Blank \\
\hline & & $4 / 24 / 2007$ & $<0.5$ & $\mu \mathrm{g} / \mathrm{L}$ & Blank \\
\hline & Hearst Sewer & $4 / 24 / 2007$ & $<1$ & $\mu \mathrm{g} / \mathrm{L}$ & Sample \\
\hline & & 9/24/2007 & $<1$ & $\mu \mathrm{g} / \mathrm{L}$ & Sample \\
\hline & & $9 / 24 / 2007$ & $<0.5$ & $\mu \mathrm{g} / \mathrm{L}$ & Split \\
\hline & Strawberry Sewer & $4 / 24 / 2007$ & $<1$ & $\mu \mathrm{g} / \mathrm{L}$ & Sample \\
\hline & & $4 / 24 / 2007$ & $<0.5$ & $\mu \mathrm{g} / \mathrm{L}$ & Split \\
\hline & & $9 / 24 / 2007$ & $<1$ & $\mu \mathrm{g} / \mathrm{L}$ & Sample \\
\hline \multirow[t]{8}{*}{ Chlorobenzene } & FIELD BLANK & $4 / 24 / 2007$ & $<1$ & $\mu \mathrm{g} / \mathrm{L}$ & Blank \\
\hline & & $4 / 24 / 2007$ & $<0.5$ & $\mu \mathrm{g} / \mathrm{L}$ & Blank \\
\hline & Hearst Sewer & $4 / 24 / 2007$ & $<1$ & $\mu \mathrm{g} / \mathrm{L}$ & Sample \\
\hline & & $9 / 24 / 2007$ & $<1$ & $\mu \mathrm{g} / \mathrm{L}$ & Sample \\
\hline & & $9 / 24 / 2007$ & $<0.5$ & $\mu \mathrm{g} / \mathrm{L}$ & Split \\
\hline & Strawberry Sewer & $4 / 24 / 2007$ & $<1$ & $\mu \mathrm{g} / \mathrm{L}$ & Sample \\
\hline & & $4 / 24 / 2007$ & $<0.5$ & $\mu \mathrm{g} / \mathrm{L}$ & Split \\
\hline & & $9 / 24 / 2007$ & $<1$ & $\mu \mathrm{g} / \mathrm{L}$ & Sample \\
\hline \multirow[t]{4}{*}{ Chloroethane } & FIELD BLANK & $4 / 24 / 2007$ & $<1$ & $\mu \mathrm{g} / \mathrm{L}$ & Blank \\
\hline & & $4 / 24 / 2007$ & $<2$ & $\mu \mathrm{g} / \mathrm{L}$ & Blank \\
\hline & Hearst Sewer & $4 / 24 / 2007$ & $<2$ & $\mu \mathrm{g} / \mathrm{L}$ & Sample \\
\hline & & 9/24/2007 & $<2$ & $\mu \mathrm{g} / \mathrm{L}$ & Sample \\
\hline
\end{tabular}




\begin{tabular}{|c|c|c|c|c|c|}
\hline \multicolumn{2}{|c|}{ Volatile Organic Compounds } & \multirow[b]{2}{*}{$\begin{array}{c}\text { Collection } \\
\text { Date }\end{array}$} & \multirow[b]{2}{*}{ Result } & \multirow[b]{2}{*}{ Units } & \multirow[b]{2}{*}{$\begin{array}{c}\text { QA } \\
\text { Type }\end{array}$} \\
\hline Analyte & Location & & & & \\
\hline \multirow[t]{4}{*}{ Chloroethane } & Hearst Sewer & 9/24/2007 & $<1$ & $\mu \mathrm{g} / \mathrm{L}$ & Split \\
\hline & Strawberry Sewer & 4/24/2007 & $<2$ & $\mu \mathrm{g} / \mathrm{L}$ & Sample \\
\hline & & $4 / 24 / 2007$ & $<1$ & $\mu \mathrm{g} / \mathrm{L}$ & Split \\
\hline & & 9/24/2007 & $<2$ & $\mu \mathrm{g} / \mathrm{L}$ & Sample \\
\hline \multirow[t]{8}{*}{ Chloroform } & FIELD BLANK & $4 / 24 / 2007$ & 2.2 & $\mu \mathrm{g} / \mathrm{L}$ & Blank \\
\hline & & 4/24/2007 & $<0.5$ & $\mu \mathrm{g} / \mathrm{L}$ & Blank \\
\hline & Hearst Sewer & $4 / 24 / 2007$ & 14 & $\mu \mathrm{g} / \mathrm{L}$ & Sample \\
\hline & & 9/24/2007 & 8.1 & $\mu \mathrm{g} / \mathrm{L}$ & Sample \\
\hline & & 9/24/2007 & 8.8 & $\mu g / L$ & Split \\
\hline & Strawberry Sewer & $4 / 24 / 2007$ & 1.5 & $\mu \mathrm{g} / \mathrm{L}$ & Sample \\
\hline & & 4/24/2007 & 1.4 & $\mu \mathrm{g} / \mathrm{L}$ & Split \\
\hline & & 9/24/2007 & 1.7 & $\mu \mathrm{g} / \mathrm{L}$ & Sample \\
\hline \multirow[t]{8}{*}{ Chloromethane } & FIELD BLANK & 4/24/2007 & 1.7 & $\mu \mathrm{g} / \mathrm{L}$ & Blank \\
\hline & & 4/24/2007 & $<2$ & $\mu \mathrm{g} / \mathrm{L}$ & Blank \\
\hline & Hearst Sewer & $4 / 24 / 2007$ & $<2$ & $\mu \mathrm{g} / \mathrm{L}$ & Sample \\
\hline & & 9/24/2007 & $<2$ & $\mu \mathrm{g} / \mathrm{L}$ & Sample \\
\hline & & 9/24/2007 & 0.91 & $\mu \mathrm{g} / \mathrm{L}$ & Split \\
\hline & Strawberry Sewer & $4 / 24 / 2007$ & $<2$ & $\mu \mathrm{g} / \mathrm{L}$ & Sample \\
\hline & & 4/24/2007 & $<0.5$ & $\mu \mathrm{g} / \mathrm{L}$ & Split \\
\hline & & 9/24/2007 & $<2$ & $\mu \mathrm{g} / \mathrm{L}$ & Sample \\
\hline \multirow[t]{6}{*}{ cis-1,2-Dichloroethene } & FIELD BLANK & $4 / 24 / 2007$ & $<1$ & $\mu \mathrm{g} / \mathrm{L}$ & Blank \\
\hline & Hearst Sewer & 4/24/2007 & $<1$ & $\mu \mathrm{g} / \mathrm{L}$ & Sample \\
\hline & & 9/24/2007 & $<1$ & $\mu g / L$ & Sample \\
\hline & & 9/24/2007 & $<0.5$ & $\mu \mathrm{g} / \mathrm{L}$ & Split \\
\hline & Strawberry Sewer & 4/24/2007 & $<1$ & $\mu \mathrm{g} / \mathrm{L}$ & Sample \\
\hline & & 9/24/2007 & $<1$ & $\mu g / L$ & Sample \\
\hline \multirow[t]{8}{*}{ cis-1,3-Dichloropropene } & FIELD BLANK & $4 / 24 / 2007$ & $<1$ & $\mu g / L$ & Blank \\
\hline & & $4 / 24 / 2007$ & $<0.5$ & $\mu \mathrm{g} / \mathrm{L}$ & Blank \\
\hline & Hearst Sewer & $4 / 24 / 2007$ & $<1$ & $\mu \mathrm{g} / \mathrm{L}$ & Sample \\
\hline & & 9/24/2007 & $<1$ & $\mu \mathrm{g} / \mathrm{L}$ & Sample \\
\hline & & 9/24/2007 & $<0.5$ & $\mu \mathrm{g} / \mathrm{L}$ & Split \\
\hline & Strawberry Sewer & 4/24/2007 & $<1$ & $\mu \mathrm{g} / \mathrm{L}$ & Sample \\
\hline & & $4 / 24 / 2007$ & $<0.5$ & $\mu \mathrm{g} / \mathrm{L}$ & Split \\
\hline & & 9/24/2007 & $<1$ & $\mu \mathrm{g} / \mathrm{L}$ & Sample \\
\hline Dibromochloromethane & FIELD BLANK & 4/24/2007 & $<0.5$ & $\mu \mathrm{g} / \mathrm{L}$ & Blank \\
\hline
\end{tabular}




\begin{tabular}{|c|c|c|c|c|c|}
\hline \multicolumn{2}{|c|}{ Volatile Organic Compounds } & \multirow[b]{2}{*}{$\begin{array}{c}\text { Collection } \\
\text { Date }\end{array}$} & \multirow[b]{2}{*}{ Result } & \multirow[b]{2}{*}{ Units } & \multirow[b]{2}{*}{$\begin{array}{c}\text { QA } \\
\text { Type }\end{array}$} \\
\hline Analyte & Location & & & & \\
\hline \multirow[t]{7}{*}{ Dibromochloromethane } & FIELD BLANK & $4 / 24 / 2007$ & $<1$ & $\mu \mathrm{g} / \mathrm{L}$ & Blank \\
\hline & Hearst Sewer & $4 / 24 / 2007$ & $<1$ & $\mu \mathrm{g} / \mathrm{L}$ & Sample \\
\hline & & 9/24/2007 & $<1$ & $\mu \mathrm{g} / \mathrm{L}$ & Sample \\
\hline & & 9/24/2007 & $<0.5$ & $\mu \mathrm{g} / \mathrm{L}$ & Split \\
\hline & Strawberry Sewer & $4 / 24 / 2007$ & $<1$ & $\mu \mathrm{g} / \mathrm{L}$ & Sample \\
\hline & & $4 / 24 / 2007$ & $<0.5$ & $\mu \mathrm{g} / \mathrm{L}$ & Split \\
\hline & & 9/24/2007 & $<1$ & $\mu \mathrm{g} / \mathrm{L}$ & Sample \\
\hline \multirow[t]{6}{*}{ Dibromomethane } & FIELD BLANK & $4 / 24 / 2007$ & $<1$ & $\mu \mathrm{g} / \mathrm{L}$ & Blank \\
\hline & Hearst Sewer & $4 / 24 / 2007$ & $<1$ & $\mu \mathrm{g} / \mathrm{L}$ & Sample \\
\hline & & 9/24/2007 & $<1$ & $\mu \mathrm{g} / \mathrm{L}$ & Sample \\
\hline & & 9/24/2007 & $<0.5$ & $\mu \mathrm{g} / \mathrm{L}$ & Split \\
\hline & Strawberry Sewer & $4 / 24 / 2007$ & $<1$ & $\mu \mathrm{g} / \mathrm{L}$ & Sample \\
\hline & & 9/24/2007 & $<1$ & $\mu \mathrm{g} / \mathrm{L}$ & Sample \\
\hline \multirow[t]{6}{*}{ Dichlorodifluoromethane } & FIELD BLANK & $4 / 24 / 2007$ & $<1$ & $\mu \mathrm{g} / \mathrm{L}$ & Blank \\
\hline & Hearst Sewer & $4 / 24 / 2007$ & $<1$ & $\mu \mathrm{g} / \mathrm{L}$ & Sample \\
\hline & & 9/24/2007 & $<1$ & $\mu \mathrm{g} / \mathrm{L}$ & Sample \\
\hline & & 9/24/2007 & $<0.5$ & $\mu \mathrm{g} / \mathrm{L}$ & Split \\
\hline & Strawberry Sewer & $4 / 24 / 2007$ & $<1$ & $\mu \mathrm{g} / \mathrm{L}$ & Sample \\
\hline & & 9/24/2007 & $<1$ & $\mu \mathrm{g} / \mathrm{L}$ & Sample \\
\hline \multirow[t]{8}{*}{ Ethylbenzene } & FIELD BLANK & $4 / 24 / 2007$ & $<0.5$ & $\mu \mathrm{g} / \mathrm{L}$ & Blank \\
\hline & & $4 / 24 / 2007$ & $<1$ & $\mu \mathrm{g} / \mathrm{L}$ & Blank \\
\hline & Hearst Sewer & $4 / 24 / 2007$ & $<1$ & $\mu \mathrm{g} / \mathrm{L}$ & Sample \\
\hline & & $9 / 24 / 2007$ & $<1$ & $\mu \mathrm{g} / \mathrm{L}$ & Sample \\
\hline & & 9/24/2007 & $<0.5$ & $\mu \mathrm{g} / \mathrm{L}$ & Split \\
\hline & Strawberry Sewer & $4 / 24 / 2007$ & $<1$ & $\mu \mathrm{g} / \mathrm{L}$ & Sample \\
\hline & & $4 / 24 / 2007$ & $<0.5$ & $\mu \mathrm{g} / \mathrm{L}$ & Split \\
\hline & & 9/24/2007 & $<1$ & $\mu \mathrm{g} / \mathrm{L}$ & Sample \\
\hline \multirow[t]{6}{*}{ Freon 113} & FIELD BLANK & $4 / 24 / 2007$ & $<1$ & $\mu \mathrm{g} / \mathrm{L}$ & Blank \\
\hline & Hearst Sewer & $4 / 24 / 2007$ & $<1$ & $\mu \mathrm{g} / \mathrm{L}$ & Sample \\
\hline & & $9 / 24 / 2007$ & $<1$ & $\mu \mathrm{g} / \mathrm{L}$ & Sample \\
\hline & & $9 / 24 / 2007$ & $<0.5$ & $\mu \mathrm{g} / \mathrm{L}$ & Split \\
\hline & Strawberry Sewer & 4/24/2007 & $<1$ & $\mu \mathrm{g} / \mathrm{L}$ & Sample \\
\hline & & $9 / 24 / 2007$ & $<1$ & $\mu \mathrm{g} / \mathrm{L}$ & Sample \\
\hline \multirow[t]{2}{*}{ Methylene chloride } & FIELD BLANK & $4 / 24 / 2007$ & $<1$ & $\mu \mathrm{g} / \mathrm{L}$ & Blank \\
\hline & & 4/24/2007 & $<0.5$ & $\mu \mathrm{g} / \mathrm{L}$ & Blank \\
\hline
\end{tabular}




\begin{tabular}{|c|c|c|c|c|c|}
\hline \multicolumn{2}{|c|}{ Volatile Organic Compounds } & \multirow[b]{2}{*}{$\begin{array}{c}\text { Collection } \\
\text { Date }\end{array}$} & \multirow[b]{2}{*}{ Result } & \multirow[b]{2}{*}{ Units } & \multirow[b]{2}{*}{$\begin{array}{c}\text { QA } \\
\text { Type }\end{array}$} \\
\hline Analyte & Location & & & & \\
\hline \multirow[t]{6}{*}{ Methylene chloride } & Hearst Sewer & 4/24/2007 & $<1$ & $\mu \mathrm{g} / \mathrm{L}$ & Sample \\
\hline & & 9/24/2007 & $<1$ & $\mu \mathrm{g} / \mathrm{L}$ & Sample \\
\hline & & 9/24/2007 & 0.6 & $\mu \mathrm{g} / \mathrm{L}$ & Split \\
\hline & Strawberry Sewer & 4/24/2007 & $<1$ & $\mu \mathrm{g} / \mathrm{L}$ & Sample \\
\hline & & 4/24/2007 & $<0.5$ & $\mu \mathrm{g} / \mathrm{L}$ & Split \\
\hline & & 9/24/2007 & $<1$ & $\mu \mathrm{g} / \mathrm{L}$ & Sample \\
\hline \multirow[t]{6}{*}{ Styrene } & FIELD BLANK & 4/24/2007 & $<1$ & $\mu \mathrm{g} / \mathrm{L}$ & Blank \\
\hline & Hearst Sewer & 4/24/2007 & $<1$ & $\mu \mathrm{g} / \mathrm{L}$ & Sample \\
\hline & & 9/24/2007 & $<1$ & $\mu \mathrm{g} / \mathrm{L}$ & Sample \\
\hline & & 9/24/2007 & $<0.5$ & $\mu \mathrm{g} / \mathrm{L}$ & Split \\
\hline & Strawberry Sewer & 4/24/2007 & $<1$ & $\mu \mathrm{g} / \mathrm{L}$ & Sample \\
\hline & & 9/24/2007 & $<1$ & $\mu \mathrm{g} / \mathrm{L}$ & Sample \\
\hline \multirow[t]{8}{*}{ Tetrachloroethene } & FIELD BLANK & 4/24/2007 & $<0.5$ & $\mu \mathrm{g} / \mathrm{L}$ & Blank \\
\hline & & 4/24/2007 & $<1$ & $\mu \mathrm{g} / \mathrm{L}$ & Blank \\
\hline & Hearst Sewer & $4 / 24 / 2007$ & $<1$ & $\mu \mathrm{g} / \mathrm{L}$ & Sample \\
\hline & & 9/24/2007 & $<1$ & $\mu \mathrm{g} / \mathrm{L}$ & Sample \\
\hline & & 9/24/2007 & $<0.5$ & $\mu \mathrm{g} / \mathrm{L}$ & Split \\
\hline & Strawberry Sewer & $4 / 24 / 2007$ & $<1$ & $\mu \mathrm{g} / \mathrm{L}$ & Sample \\
\hline & & 4/24/2007 & $<0.5$ & $\mu \mathrm{g} / \mathrm{L}$ & Split \\
\hline & & 9/24/2007 & $<1$ & $\mu \mathrm{g} / \mathrm{L}$ & Sample \\
\hline \multirow[t]{8}{*}{ Toluene } & FIELD BLANK & $4 / 24 / 2007$ & $<0.5$ & $\mu \mathrm{g} / \mathrm{L}$ & Blank \\
\hline & & $4 / 24 / 2007$ & $<1$ & $\mu \mathrm{g} / \mathrm{L}$ & Blank \\
\hline & Hearst Sewer & $4 / 24 / 2007$ & $<1$ & $\mu \mathrm{g} / \mathrm{L}$ & Sample \\
\hline & & 9/24/2007 & $<1$ & $\mu \mathrm{g} / \mathrm{L}$ & Sample \\
\hline & & 9/24/2007 & $<0.5$ & $\mu \mathrm{g} / \mathrm{L}$ & Split \\
\hline & Strawberry Sewer & $4 / 24 / 2007$ & $<1$ & $\mu \mathrm{g} / \mathrm{L}$ & Sample \\
\hline & & $4 / 24 / 2007$ & $<0.5$ & $\mu \mathrm{g} / \mathrm{L}$ & Split \\
\hline & & 9/24/2007 & $<1$ & $\mu \mathrm{g} / \mathrm{L}$ & Sample \\
\hline \multirow[t]{6}{*}{ Total xylene isomers } & FIELD BLANK & 4/24/2007 & $<2$ & $\mu \mathrm{g} / \mathrm{L}$ & Blank \\
\hline & Hearst Sewer & 4/24/2007 & $<2$ & $\mu \mathrm{g} / \mathrm{L}$ & Sample \\
\hline & & 9/24/2007 & $<2$ & $\mu \mathrm{g} / \mathrm{L}$ & Sample \\
\hline & & 9/24/2007 & $<0.5$ & $\mu \mathrm{g} / \mathrm{L}$ & Split \\
\hline & Strawberry Sewer & $4 / 24 / 2007$ & $<2$ & $\mu \mathrm{g} / \mathrm{L}$ & Sample \\
\hline & & 9/24/2007 & $<2$ & $\mu \mathrm{g} / \mathrm{L}$ & Sample \\
\hline trans-1,2-Dichloroethene & FIELD BLANK & 4/24/2007 & $<0.5$ & $\mu \mathrm{g} / \mathrm{L}$ & Blank \\
\hline
\end{tabular}




\begin{tabular}{|c|c|c|c|c|c|}
\hline \multicolumn{2}{|c|}{ Volatile Organic Compounds } & \multirow[b]{2}{*}{$\begin{array}{c}\text { Collection } \\
\text { Date }\end{array}$} & \multirow[b]{2}{*}{ Result } & \multirow[b]{2}{*}{ Units } & \multirow[b]{2}{*}{$\begin{array}{c}\text { QA } \\
\text { Type }\end{array}$} \\
\hline Analyte & Location & & & & \\
\hline \multirow[t]{7}{*}{ trans-1,2-Dichloroethene } & FIELD BLANK & 4/24/2007 & $<1$ & $\mu \mathrm{g} / \mathrm{L}$ & Blank \\
\hline & Hearst Sewer & $4 / 24 / 2007$ & $<1$ & $\mu \mathrm{g} / \mathrm{L}$ & Sample \\
\hline & & 9/24/2007 & $<1$ & $\mu \mathrm{g} / \mathrm{L}$ & Sample \\
\hline & & 9/24/2007 & $<0.5$ & $\mu \mathrm{g} / \mathrm{L}$ & Split \\
\hline & Strawberry Sewer & 4/24/2007 & $<1$ & $\mu \mathrm{g} / \mathrm{L}$ & Sample \\
\hline & & 4/24/2007 & $<0.5$ & $\mu \mathrm{g} / \mathrm{L}$ & Split \\
\hline & & 9/24/2007 & $<1$ & $\mu \mathrm{g} / \mathrm{L}$ & Sample \\
\hline \multirow[t]{8}{*}{ trans-1,3-Dichloropropene } & FIELD BLANK & 4/24/2007 & $<1$ & $\mu \mathrm{g} / \mathrm{L}$ & Blank \\
\hline & & 4/24/2007 & $<0.5$ & $\mu \mathrm{g} / \mathrm{L}$ & Blank \\
\hline & Hearst Sewer & $4 / 24 / 2007$ & $<1$ & $\mu \mathrm{g} / \mathrm{L}$ & Sample \\
\hline & & 9/24/2007 & $<1$ & $\mu \mathrm{g} / \mathrm{L}$ & Sample \\
\hline & & 9/24/2007 & $<0.5$ & $\mu \mathrm{g} / \mathrm{L}$ & Split \\
\hline & Strawberry Sewer & 4/24/2007 & $<1$ & $\mu \mathrm{g} / \mathrm{L}$ & Sample \\
\hline & & 4/24/2007 & $<0.5$ & $\mu \mathrm{g} / \mathrm{L}$ & Split \\
\hline & & 9/24/2007 & $<1$ & $\mu \mathrm{g} / \mathrm{L}$ & Sample \\
\hline \multirow[t]{8}{*}{ Trichloroethene } & FIELD BLANK & $4 / 24 / 2007$ & $<0.5$ & $\mu \mathrm{g} / \mathrm{L}$ & Blank \\
\hline & & $4 / 24 / 2007$ & $<0.5$ & $\mu \mathrm{g} / \mathrm{L}$ & Blank \\
\hline & Hearst Sewer & $4 / 24 / 2007$ & $<0.5$ & $\mu \mathrm{g} / \mathrm{L}$ & Sample \\
\hline & & 9/24/2007 & $<0.5$ & $\mu \mathrm{g} / \mathrm{L}$ & Sample \\
\hline & & 9/24/2007 & $<0.5$ & $\mu \mathrm{g} / \mathrm{L}$ & Split \\
\hline & Strawberry Sewer & $4 / 24 / 2007$ & $<0.5$ & $\mu \mathrm{g} / \mathrm{L}$ & Sample \\
\hline & & $4 / 24 / 2007$ & $<0.5$ & $\mu \mathrm{g} / \mathrm{L}$ & Split \\
\hline & & 9/24/2007 & $<0.5$ & $\mu \mathrm{g} / \mathrm{L}$ & Sample \\
\hline \multirow[t]{8}{*}{ Trichlorofluoromethane } & FIELD BLANK & 4/24/2007 & 2.7 & $\mu \mathrm{g} / \mathrm{L}$ & Blank \\
\hline & & $4 / 24 / 2007$ & 2.2 & $\mu g / L$ & Blank \\
\hline & Hearst Sewer & $4 / 24 / 2007$ & $<1$ & $\mu \mathrm{g} / \mathrm{L}$ & Sample \\
\hline & & 9/24/2007 & $<1$ & $\mu \mathrm{g} / \mathrm{L}$ & Sample \\
\hline & & 9/24/2007 & $<0.5$ & $\mu \mathrm{g} / \mathrm{L}$ & Split \\
\hline & Strawberry Sewer & 4/24/2007 & $<1$ & $\mu \mathrm{g} / \mathrm{L}$ & Sample \\
\hline & & $4 / 24 / 2007$ & $<0.5$ & $\mu \mathrm{g} / \mathrm{L}$ & Split \\
\hline & & 9/24/2007 & $<1$ & $\mu \mathrm{g} / \mathrm{L}$ & Sample \\
\hline \multirow[t]{4}{*}{ Vinyl acetate } & FIELD BLANK & 4/24/2007 & $<20$ & $\mu \mathrm{g} / \mathrm{L}$ & Blank \\
\hline & Hearst Sewer & $4 / 24 / 2007$ & $<20$ & $\mu \mathrm{g} / \mathrm{L}$ & Sample \\
\hline & & 9/24/2007 & $<20$ & $\mu \mathrm{g} / \mathrm{L}$ & Sample \\
\hline & & 9/24/2007 & $<10$ & $\mu \mathrm{g} / \mathrm{L}$ & Split \\
\hline
\end{tabular}




\begin{tabular}{|l|l|c|c|c|c|}
\hline \multicolumn{2}{|c|}{ Volatile Organic Compounds } & & & \\
\hline Analyte & Location & $\begin{array}{c}\text { Collection } \\
\text { Date }\end{array}$ & Result & Units & $\begin{array}{c}\text { QA } \\
\text { Type }\end{array}$ \\
\hline Vinyl acetate & Strawberry Sewer & $4 / 24 / 2007$ & $<20$ & $\mu \mathrm{g} / \mathrm{L}$ & Sample \\
\hline & & $9 / 24 / 2007$ & $<20$ & $\mu \mathrm{g} / \mathrm{L}$ & Sample \\
\hline Vinyl chloride & FIELD BLANK & $4 / 24 / 2007$ & $<0.5$ & $\mu \mathrm{g} / \mathrm{L}$ & Blank \\
\hline & & $4 / 24 / 2007$ & $<1$ & $\mu \mathrm{g} / \mathrm{L}$ & Blank \\
\hline & Hearst Sewer & $4 / 24 / 2007$ & $<1$ & $\mu \mathrm{g} / \mathrm{L}$ & Sample \\
\hline & & $9 / 24 / 2007$ & $<1$ & $\mu \mathrm{g} / \mathrm{L}$ & Sample \\
\hline & & $9 / 24 / 2007$ & $<0.5$ & $\mu \mathrm{g} / \mathrm{L}$ & Split \\
\hline & Strawberry Sewer & $4 / 24 / 2007$ & $<1$ & $\mu \mathrm{g} / \mathrm{L}$ & Sample \\
\hline & & $4 / 24 / 2007$ & $<0.5$ & $\mu \mathrm{g} / \mathrm{L}$ & Split \\
\hline & & $9 / 24 / 2007$ & $<1$ & $\mu \mathrm{g} / \mathrm{L}$ & Sample \\
\hline
\end{tabular}




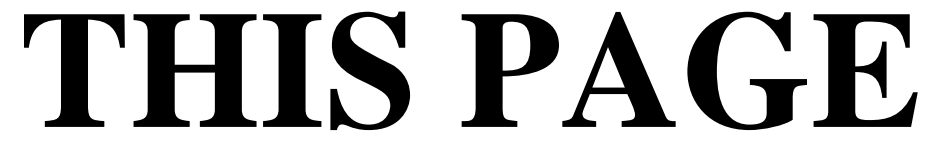

INTENTIONALLY LEFT BLANK 


\begin{tabular}{|c|c|c|c|c|c|}
\hline \multicolumn{2}{|c|}{ Metals and Minerals } & \multirow[b]{2}{*}{$\begin{array}{c}\text { Collection } \\
\text { Date }\end{array}$} & \multirow[b]{2}{*}{ Result } & \multirow[b]{2}{*}{ Units } & \multirow[b]{2}{*}{ QA Type } \\
\hline Analyte & Location & & & & \\
\hline \multirow[t]{12}{*}{ Cadmium } & $25 \mathrm{FTU}$ & $2 / 13 / 2007$ & $<0.01$ & $\mathrm{mg} / \mathrm{L}$ & Sample \\
\hline & & 2/13/2007 & $<0.01$ & $\mathrm{mg} / \mathrm{L}$ & Split \\
\hline & & $9 / 10 / 2007$ & $<0.01$ & $\mathrm{mg} / \mathrm{L}$ & Sample \\
\hline & & 9/10/2007 & $<0.005$ & $\mathrm{mg} / \mathrm{L}$ & Split \\
\hline & 77 FTU & $1 / 30 / 2007$ & $<0.02$ & $\mathrm{mg} / \mathrm{L}$ & Sample \\
\hline & & $1 / 30 / 2007$ & $<0.01$ & $\mathrm{mg} / \mathrm{L}$ & Split \\
\hline & & $7 / 17 / 2007$ & $<0.01$ & $\mathrm{mg} / \mathrm{L}$ & Sample \\
\hline & & $7 / 17 / 2007$ & $<0.01$ & $\mathrm{mg} / \mathrm{L}$ & Split \\
\hline & & 9/11/2007 & $<0.01$ & $\mathrm{mg} / \mathrm{L}$ & Sample \\
\hline & & $9 / 11 / 2007$ & $<0.005$ & $\mathrm{mg} / \mathrm{L}$ & Split \\
\hline & TRAVEL BLANK & $1 / 30 / 2007$ & $<0.01$ & $\mathrm{mg} / \mathrm{L}$ & Blank \\
\hline & & $1 / 30 / 2007$ & $<0.01$ & $\mathrm{mg} / \mathrm{L}$ & Blank \\
\hline \multirow[t]{12}{*}{ Chromium } & $25 \mathrm{FTU}$ & 2/13/2007 & $<0.05$ & $\mathrm{mg} / \mathrm{L}$ & Sample \\
\hline & & $2 / 13 / 2007$ & $<0.05$ & $\mathrm{mg} / \mathrm{L}$ & Split \\
\hline & & 9/10/2007 & $<0.05$ & $\mathrm{mg} / \mathrm{L}$ & Sample \\
\hline & & $9 / 10 / 2007$ & $<0.025$ & $\mathrm{mg} / \mathrm{L}$ & Split \\
\hline & 77 FTU & $1 / 30 / 2007$ & $<0.1$ & $\mathrm{mg} / \mathrm{L}$ & Sample \\
\hline & & $1 / 30 / 2007$ & $<0.05$ & $\mathrm{mg} / \mathrm{L}$ & Split \\
\hline & & 7/17/2007 & $<0.05$ & $\mathrm{mg} / \mathrm{L}$ & Sample \\
\hline & & 7/17/2007 & $<0.05$ & $\mathrm{mg} / \mathrm{L}$ & Split \\
\hline & & $9 / 11 / 2007$ & $<0.05$ & $\mathrm{mg} / \mathrm{L}$ & Sample \\
\hline & & 9/11/2007 & $<0.025$ & $\mathrm{mg} / \mathrm{L}$ & Split \\
\hline & TRAVEL BLANK & 1/30/2007 & $<0.05$ & $\mathrm{mg} / \mathrm{L}$ & Blank \\
\hline & & $1 / 30 / 2007$ & $<0.05$ & $\mathrm{mg} / \mathrm{L}$ & Blank \\
\hline \multirow[t]{10}{*}{ Copper } & $25 \mathrm{FTU}$ & 2/13/2007 & 0.14 & $\mathrm{mg} / \mathrm{L}$ & Sample \\
\hline & & 2/13/2007 & 0.3 & $\mathrm{mg} / \mathrm{L}$ & Split \\
\hline & & 9/10/2007 & 0.21 & $\mathrm{mg} / \mathrm{L}$ & Sample \\
\hline & & 9/10/2007 & 0.19 & $\mathrm{mg} / \mathrm{L}$ & Split \\
\hline & 77 FTU & $1 / 30 / 2007$ & $<0.1$ & $\mathrm{mg} / \mathrm{L}$ & Sample \\
\hline & & $1 / 30 / 2007$ & 0.089 & $\mathrm{mg} / \mathrm{L}$ & Split \\
\hline & & $7 / 17 / 2007$ & 0.38 & $\mathrm{mg} / \mathrm{L}$ & Sample \\
\hline & & $7 / 17 / 2007$ & 0.21 & $\mathrm{mg} / \mathrm{L}$ & Split \\
\hline & & 9/11/2007 & 0.28 & $\mathrm{mg} / \mathrm{L}$ & Sample \\
\hline & & 9/11/2007 & 0.25 & $\mathrm{mg} / \mathrm{L}$ & Split \\
\hline
\end{tabular}




\begin{tabular}{|c|c|c|c|c|c|}
\hline \multicolumn{2}{|c|}{ Metals and Minerals } & \multirow[b]{2}{*}{$\begin{array}{c}\text { Collection } \\
\text { Date }\end{array}$} & \multirow[b]{2}{*}{ Result } & \multirow[b]{2}{*}{ Units } & \multirow[b]{2}{*}{ QA Type } \\
\hline Analyte & Location & & & & \\
\hline \multirow[t]{2}{*}{ Copper } & TRAVEL BLANK & $1 / 30 / 2007$ & $<0.05$ & $\mathrm{mg} / \mathrm{L}$ & Blank \\
\hline & & $1 / 30 / 2007$ & $<0.05$ & $\mathrm{mg} / \mathrm{L}$ & Blank \\
\hline \multirow[t]{12}{*}{ Lead } & $25 \mathrm{FTU}$ & 2/13/2007 & $<0.01$ & $\mathrm{mg} / \mathrm{L}$ & Sample \\
\hline & & $2 / 13 / 2007$ & $<0.1$ & $\mathrm{mg} / \mathrm{L}$ & Split \\
\hline & & 9/10/2007 & $<0.1$ & $\mathrm{mg} / \mathrm{L}$ & Sample \\
\hline & & 9/10/2007 & $<0.05$ & $\mathrm{mg} / \mathrm{L}$ & Split \\
\hline & 77 FTU & $1 / 30 / 2007$ & $<0.02$ & $\mathrm{mg} / \mathrm{L}$ & Sample \\
\hline & & $1 / 30 / 2007$ & $<0.1$ & $\mathrm{mg} / \mathrm{L}$ & Split \\
\hline & & $7 / 17 / 2007$ & $<0.1$ & $\mathrm{mg} / \mathrm{L}$ & Sample \\
\hline & & $7 / 17 / 2007$ & $<0.01$ & $\mathrm{mg} / \mathrm{L}$ & Split \\
\hline & & 9/11/2007 & $<0.1$ & $\mathrm{mg} / \mathrm{L}$ & Sample \\
\hline & & 9/11/2007 & $<0.05$ & $\mathrm{mg} / \mathrm{L}$ & Split \\
\hline & TRAVEL BLANK & $1 / 30 / 2007$ & $<0.1$ & $\mathrm{mg} / \mathrm{L}$ & Blank \\
\hline & & $1 / 30 / 2007$ & $<0.01$ & $\mathrm{mg} / \mathrm{L}$ & Blank \\
\hline \multirow[t]{12}{*}{ Nickel } & $25 \mathrm{FTU}$ & $2 / 13 / 2007$ & $<0.01$ & $\mathrm{mg} / \mathrm{L}$ & Sample \\
\hline & & 2/13/2007 & $<0.1$ & $\mathrm{mg} / \mathrm{L}$ & Split \\
\hline & & 9/10/2007 & $<0.1$ & $\mathrm{mg} / \mathrm{L}$ & Sample \\
\hline & & 9/10/2007 & $<0.05$ & $\mathrm{mg} / \mathrm{L}$ & Split \\
\hline & 77 FTU & $1 / 30 / 2007$ & 0.021 & $\mathrm{mg} / \mathrm{L}$ & Sample \\
\hline & & $1 / 30 / 2007$ & $<0.1$ & $\mathrm{mg} / \mathrm{L}$ & Split \\
\hline & & $7 / 17 / 2007$ & 0.57 & $\mathrm{mg} / \mathrm{L}$ & Sample \\
\hline & & $7 / 17 / 2007$ & 0.28 & $\mathrm{mg} / \mathrm{L}$ & Split \\
\hline & & 9/11/2007 & 0.11 & $\mathrm{mg} / \mathrm{L}$ & Sample \\
\hline & & 9/11/2007 & 0.1 & $\mathrm{mg} / \mathrm{L}$ & Split \\
\hline & TRAVEL BLANK & $1 / 30 / 2007$ & $<0.1$ & $\mathrm{mg} / \mathrm{L}$ & Blank \\
\hline & & $1 / 30 / 2007$ & 0.024 & $\mathrm{mg} / \mathrm{L}$ & Blank \\
\hline \multirow[t]{9}{*}{ Silver } & $25 \mathrm{FTU}$ & $2 / 13 / 2007$ & $<0.05$ & $\mathrm{mg} / \mathrm{L}$ & Sample \\
\hline & & 2/13/2007 & $<0.05$ & $\mathrm{mg} / \mathrm{L}$ & Split \\
\hline & & 9/10/2007 & $<0.05$ & $\mathrm{mg} / \mathrm{L}$ & Sample \\
\hline & & 9/10/2007 & $<0.025$ & $\mathrm{mg} / \mathrm{L}$ & Split \\
\hline & $77 \mathrm{FTU}$ & $1 / 30 / 2007$ & $<0.1$ & $\mathrm{mg} / \mathrm{L}$ & Sample \\
\hline & & $1 / 30 / 2007$ & $<0.05$ & $\mathrm{mg} / \mathrm{L}$ & Split \\
\hline & & $7 / 17 / 2007$ & $<0.05$ & $\mathrm{mg} / \mathrm{L}$ & Sample \\
\hline & & $7 / 17 / 2007$ & $<0.05$ & $\mathrm{mg} / \mathrm{L}$ & Split \\
\hline & & 9/11/2007 & $<0.05$ & $\mathrm{mg} / \mathrm{L}$ & Sample \\
\hline
\end{tabular}




\begin{tabular}{|l|l|c|c|c|c|}
\hline \multicolumn{2}{|c|}{ Metals and Minerals } & \multicolumn{2}{c|}{} & \\
\hline Analyte & Location & $\begin{array}{c}\text { Collection } \\
\text { Date }\end{array}$ & Result & Units & QA Type \\
\hline Silver & 77 FTU & $9 / 11 / 2007$ & $<0.025$ & $\mathrm{mg} / \mathrm{L}$ & Split \\
\hline & TRAVEL BLANK & $1 / 30 / 2007$ & $<0.05$ & $\mathrm{mg} / \mathrm{L}$ & Blank \\
\hline & & $1 / 30 / 2007$ & $<0.05$ & $\mathrm{mg} / \mathrm{L}$ & Blank \\
\hline Zinc & 25 FTU & $2 / 13 / 2007$ & $<0.05$ & $\mathrm{mg} / \mathrm{L}$ & Sample \\
\hline & & $2 / 13 / 2007$ & $<0.05$ & $\mathrm{mg} / \mathrm{L}$ & Split \\
\hline & & $9 / 10 / 2007$ & $<0.05$ & $\mathrm{mg} / \mathrm{L}$ & Sample \\
\hline & & $9 / 10 / 2007$ & $<0.025$ & $\mathrm{mg} / \mathrm{L}$ & Split \\
\hline & 77 FTU & $1 / 30 / 2007$ & $<0.1$ & $\mathrm{mg} / \mathrm{L}$ & Sample \\
\hline & & $1 / 30 / 2007$ & 0.059 & $\mathrm{mg} / \mathrm{L}$ & Split \\
\hline & & $7 / 17 / 2007$ & 0.071 & $\mathrm{mg} / \mathrm{L}$ & Sample \\
\hline & & $7 / 17 / 2007$ & 0.055 & $\mathrm{mg} / \mathrm{L}$ & Split \\
\hline & & $9 / 11 / 2007$ & $<0.05$ & $\mathrm{mg} / \mathrm{L}$ & Sample \\
\hline & & $9 / 11 / 2007$ & $<0.025$ & $\mathrm{mg} / \mathrm{L}$ & Split \\
\hline & TRAVEL BLANK & $1 / 30 / 2007$ & $<0.05$ & $\mathrm{mg} / \mathrm{L}$ & Blank \\
\hline & & $1 / 30 / 2007$ & $<0.05$ & $\mathrm{mg} / \mathrm{L}$ & Blank \\
\hline
\end{tabular}




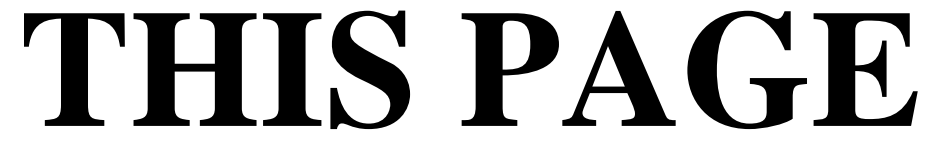

INTENTIONALLY LEFT BLANK 


\begin{tabular}{|c|c|c|c|c|c|c|c|}
\hline \multicolumn{2}{|c|}{ Radiological Activity } & \multirow[b]{2}{*}{$\begin{array}{c}\text { Collection } \\
\text { Date }\end{array}$} & \multicolumn{2}{|c|}{ S.I. } & \multicolumn{2}{|c|}{ Conventional } & \multirow[b]{2}{*}{$\begin{array}{c}\text { QA } \\
\text { Type }\end{array}$} \\
\hline Analyte & Location & & Result & Units & Result & Units & \\
\hline \multirow[t]{5}{*}{ Actinium 228} & Building 69 & $10 / 17 / 2007$ & 0.014 & $\mathrm{~Bq} / \mathrm{g}$ & 0.37 & $\mathrm{pCi} / \mathrm{g}$ & Sample \\
\hline & & $10 / 17 / 2007$ & 0.015 & $\mathrm{~Bq} / \mathrm{g}$ & 0.41 & $\mathrm{pCi} / \mathrm{g}$ & Split \\
\hline & Building 80 & $10 / 17 / 2007$ & 0.028 & $\mathrm{~Bq} / \mathrm{g}$ & 0.75 & $\mathrm{pCi} / \mathrm{g}$ & Sample \\
\hline & Building 85 & $10 / 17 / 2007$ & 0.021 & $\mathrm{~Bq} / \mathrm{g}$ & 0.56 & $\mathrm{pCi} / \mathrm{g}$ & Sample \\
\hline & ENV-B13C & $10 / 17 / 2007$ & 0.032 & $\mathrm{~Bq} / \mathrm{g}$ & 0.85 & $\mathrm{pCi} / \mathrm{g}$ & Sample \\
\hline $\begin{array}{l}\text { Americium } \\
241\end{array}$ & Building 80 & $10 / 17 / 2007$ & $<0.0011$ & $\mathrm{~Bq} / \mathrm{g}$ & $<0.029$ & $\mathrm{pCi} / \mathrm{g}$ & Sample \\
\hline \multirow[t]{5}{*}{ Cesium 137} & Building 69 & $10 / 17 / 2007$ & $<0.00072$ & $\mathrm{~Bq} / \mathrm{g}$ & $<0.019$ & $\mathrm{pCi} / \mathrm{g}$ & Sample \\
\hline & & $10 / 17 / 2007$ & $<0.00076$ & $\mathrm{~Bq} / \mathrm{g}$ & $<0.021$ & $\mathrm{pCi} / \mathrm{g}$ & Split \\
\hline & Building 80 & $10 / 17 / 2007$ & 0.0042 & $\mathrm{~Bq} / \mathrm{g}$ & 0.11 & $\mathrm{pCi} / \mathrm{g}$ & Sample \\
\hline & Building 85 & $10 / 17 / 2007$ & 0.0011 & $\mathrm{~Bq} / \mathrm{g}$ & 0.03 & $\mathrm{pCi} / \mathrm{g}$ & Sample \\
\hline & ENV-B13C & $10 / 17 / 2007$ & 0.014 & $\mathrm{~Bq} / \mathrm{g}$ & 0.38 & $\mathrm{pCi} / \mathrm{g}$ & Sample \\
\hline Cobalt 60 & Building 80 & $10 / 17 / 2007$ & $<0.001$ & $\mathrm{~Bq} / \mathrm{g}$ & $<0.028$ & $\mathrm{pCi} / \mathrm{g}$ & Sample \\
\hline \multirow[t]{5}{*}{ Gross alpha } & Building 69 & $10 / 17 / 2007$ & 0.33 & $\mathrm{~Bq} / \mathrm{g}$ & 8.8 & $\mathrm{pCi} / \mathrm{g}$ & Sample \\
\hline & & $10 / 17 / 2007$ & 0.065 & $\mathrm{~Bq} / \mathrm{g}$ & 1.8 & $\mathrm{pCi} / \mathrm{g}$ & Split \\
\hline & Building 80 & $10 / 17 / 2007$ & 0.31 & $\mathrm{~Bq} / \mathrm{g}$ & 8.4 & $\mathrm{pCi} / \mathrm{g}$ & Sample \\
\hline & Building 85 & $10 / 17 / 2007$ & 0.33 & $\mathrm{~Bq} / \mathrm{g}$ & 9 & $\mathrm{pCi} / \mathrm{g}$ & Sample \\
\hline & ENV-B13C & $10 / 17 / 2007$ & 0.39 & $\mathrm{~Bq} / \mathrm{g}$ & 10 & $\mathrm{pCi} / \mathrm{g}$ & Sample \\
\hline \multirow[t]{5}{*}{ Gross beta } & Building 69 & $10 / 17 / 2007$ & 0.35 & $\mathrm{~Bq} / \mathrm{g}$ & 9.5 & $\mathrm{pCi} / \mathrm{g}$ & Sample \\
\hline & & $10 / 17 / 2007$ & 0.096 & $\mathrm{~Bq} / \mathrm{g}$ & 2.6 & $\mathrm{pCi} / \mathrm{g}$ & Split \\
\hline & Building 80 & $10 / 17 / 2007$ & 0.89 & $\mathrm{~Bq} / \mathrm{g}$ & 24 & $\mathrm{pCi} / \mathrm{g}$ & Sample \\
\hline & Building 85 & $10 / 17 / 2007$ & 0.55 & $\mathrm{~Bq} / \mathrm{g}$ & 15 & $\mathrm{pCi} / \mathrm{g}$ & Sample \\
\hline & ENV-B13C & $10 / 17 / 2007$ & 0.89 & $\mathrm{~Bq} / \mathrm{g}$ & 24 & $\mathrm{pCi} / \mathrm{g}$ & Sample \\
\hline \multirow[t]{5}{*}{ Lead 214} & Building 69 & $10 / 17 / 2007$ & 0.015 & $\mathrm{~Bq} / \mathrm{g}$ & 0.42 & $\mathrm{pCi} / \mathrm{g}$ & Sample \\
\hline & & $10 / 17 / 2007$ & 0.016 & $\mathrm{~Bq} / \mathrm{g}$ & 0.44 & $\mathrm{pCi} / \mathrm{g}$ & Split \\
\hline & Building 80 & $10 / 17 / 2007$ & 0.024 & $\mathrm{~Bq} / \mathrm{g}$ & 0.65 & $\mathrm{pCi} / \mathrm{g}$ & Sample \\
\hline & Building 85 & $10 / 17 / 2007$ & 0.021 & $\mathrm{~Bq} / \mathrm{g}$ & 0.58 & $\mathrm{pCi} / \mathrm{g}$ & Sample \\
\hline & ENV-B13C & $10 / 17 / 2007$ & 0.026 & $\mathrm{~Bq} / \mathrm{g}$ & 0.7 & $\mathrm{pCi} / \mathrm{g}$ & Sample \\
\hline \multirow[t]{5}{*}{ Potassium 40} & Building 69 & $10 / 17 / 2007$ & 0.28 & $\mathrm{~Bq} / \mathrm{g}$ & 7.7 & $\mathrm{pCi} / \mathrm{g}$ & Sample \\
\hline & & $10 / 17 / 2007$ & 0.29 & $\mathrm{~Bq} / \mathrm{g}$ & 7.8 & $\mathrm{pCi} / \mathrm{g}$ & Split \\
\hline & Building 80 & $10 / 17 / 2007$ & 0.59 & $\mathrm{~Bq} / \mathrm{g}$ & 16 & $\mathrm{pCi} / \mathrm{g}$ & Sample \\
\hline & Building 85 & $10 / 17 / 2007$ & 0.43 & $\mathrm{~Bq} / \mathrm{g}$ & 12 & $\mathrm{pCi} / \mathrm{g}$ & Sample \\
\hline & ENV-B13C & $10 / 17 / 2007$ & 0.65 & $\mathrm{~Bq} / \mathrm{g}$ & 18 & $\mathrm{pCi} / \mathrm{g}$ & Sample \\
\hline Radium 226 & Building 69 & $10 / 17 / 2007$ & 0.015 & $\mathrm{~Bq} / \mathrm{g}$ & 0.41 & $\mathrm{pCi} / \mathrm{g}$ & Sample \\
\hline
\end{tabular}




\begin{tabular}{|c|c|c|c|c|c|c|c|}
\hline \multicolumn{2}{|c|}{ Radiological Activity } & \multirow[b]{2}{*}{$\begin{array}{l}\text { Collection } \\
\text { Date }\end{array}$} & \multicolumn{2}{|c|}{ S.I. } & \multicolumn{2}{|c|}{ Conventional } & \multirow[b]{2}{*}{$\begin{array}{c}\text { QA } \\
\text { Type } \\
\end{array}$} \\
\hline Analyte & Location & & Result & Units & Result & Units & \\
\hline \multirow[t]{4}{*}{ Radium 226} & Building 69 & $10 / 17 / 2007$ & 0.014 & $\mathrm{~Bq} / \mathrm{g}$ & 0.38 & $\mathrm{pCi} / \mathrm{g}$ & Split \\
\hline & Building 80 & $10 / 17 / 2007$ & 0.02 & $\mathrm{~Bq} / \mathrm{g}$ & 0.55 & $\mathrm{pCi} / \mathrm{g}$ & Sample \\
\hline & Building 85 & $10 / 17 / 2007$ & 0.02 & $\mathrm{~Bq} / \mathrm{g}$ & 0.53 & $\mathrm{pCi} / \mathrm{g}$ & Sample \\
\hline & ENV-B13C & $10 / 17 / 2007$ & 0.024 & $\mathrm{~Bq} / \mathrm{g}$ & 0.65 & $\mathrm{pCi} / \mathrm{g}$ & Sample \\
\hline \multirow[t]{5}{*}{ Radium 228} & Building 69 & $10 / 17 / 2007$ & 0.014 & $\mathrm{~Bq} / \mathrm{g}$ & 0.37 & $\mathrm{pCi} / \mathrm{g}$ & Sample \\
\hline & & $10 / 17 / 2007$ & 0.015 & $\mathrm{~Bq} / \mathrm{g}$ & 0.41 & $\mathrm{pCi} / \mathrm{g}$ & Split \\
\hline & Building 80 & $10 / 17 / 2007$ & 0.028 & $\mathrm{~Bq} / \mathrm{g}$ & 0.75 & $\mathrm{pCi} / \mathrm{g}$ & Sample \\
\hline & Building 85 & $10 / 17 / 2007$ & 0.021 & $\mathrm{~Bq} / \mathrm{g}$ & 0.56 & $\mathrm{pCi} / \mathrm{g}$ & Sample \\
\hline & ENV-B13C & $10 / 17 / 2007$ & 0.032 & $\mathrm{~Bq} / \mathrm{g}$ & 0.85 & $\mathrm{pCi} / \mathrm{g}$ & Sample \\
\hline \multirow[t]{5}{*}{ Tritium } & Building 69 & $10 / 17 / 2007$ & $<0.0064$ & $\mathrm{~Bq} / \mathrm{g}$ & $<0.17$ & $\mathrm{pCi} / \mathrm{g}$ & Sample \\
\hline & & $10 / 17 / 2007$ & $<0.0052$ & $\mathrm{~Bq} / \mathrm{g}$ & $<0.14$ & $\mathrm{pCi} / \mathrm{g}$ & Split \\
\hline & Building 80 & $10 / 17 / 2007$ & $<0.0067$ & $\mathrm{~Bq} / \mathrm{g}$ & $<0.18$ & $\mathrm{pCi} / \mathrm{g}$ & Sample \\
\hline & Building 85 & $10 / 17 / 2007$ & $<0.0068$ & $\mathrm{~Bq} / \mathrm{g}$ & $<0.18$ & $\mathrm{pCi} / \mathrm{g}$ & Sample \\
\hline & ENV-B13C & $10 / 17 / 2007$ & $<0.0066$ & $\mathrm{~Bq} / \mathrm{g}$ & $<0.18$ & $\mathrm{pCi} / \mathrm{g}$ & Sample \\
\hline \multirow[t]{5}{*}{ Uranium 238} & Building 69 & $10 / 17 / 2007$ & 0.012 & $\mathrm{~Bq} / \mathrm{g}$ & 0.33 & $\mathrm{pCi} / \mathrm{g}$ & Sample \\
\hline & & $10 / 17 / 2007$ & 0.02 & $\mathrm{~Bq} / \mathrm{g}$ & 0.55 & $\mathrm{pCi} / \mathrm{g}$ & Split \\
\hline & Building 80 & $10 / 17 / 2007$ & $<0.01$ & $\mathrm{~Bq} / \mathrm{g}$ & $<0.28$ & $\mathrm{pCi} / \mathrm{g}$ & Sample \\
\hline & Building 85 & $10 / 17 / 2007$ & $<0.021$ & $\mathrm{~Bq} / \mathrm{g}$ & $<0.58$ & $\mathrm{pCi} / \mathrm{g}$ & Sample \\
\hline & ENV-B13C & $10 / 17 / 2007$ & 0.022 & $\mathrm{~Bq} / \mathrm{g}$ & 0.6 & $\mathrm{pCi} / \mathrm{g}$ & Sample \\
\hline
\end{tabular}




\begin{tabular}{|c|c|c|c|c|c|}
\hline \multicolumn{2}{|c|}{ General Indicator Parameters } & \multirow[b]{2}{*}{$\begin{array}{c}\text { Collection } \\
\text { Date }\end{array}$} & \multirow[b]{2}{*}{ Result } & \multirow[b]{2}{*}{ Units } & \multirow[b]{2}{*}{ QA Type } \\
\hline Analyte & Location & & & & \\
\hline \multirow[t]{5}{*}{ Moisture by weight } & Building 69 & $10 / 17 / 2007$ & 22 & $\%$ & Sample \\
\hline & & $10 / 17 / 2007$ & 23 & $\%$ & Split \\
\hline & Building 80 & $10 / 17 / 2007$ & 25 & $\%$ & Sample \\
\hline & Building 85 & $10 / 17 / 2007$ & 21 & $\%$ & Sample \\
\hline & ENV-B13C & $10 / 17 / 2007$ & 20 & $\%$ & Sample \\
\hline \multirow[t]{5}{*}{$\mathrm{pH}$} & Building 69 & $10 / 17 / 2007$ & 7.3 & S.U. & Sample \\
\hline & & $10 / 17 / 2007$ & 7.3 & S.U. & Split \\
\hline & Building 80 & $10 / 17 / 2007$ & 7 & S.U. & Sample \\
\hline & Building 85 & $10 / 17 / 2007$ & 7.4 & S.U. & Sample \\
\hline & ENV-B13C & $10 / 17 / 2007$ & 5.4 & S.U. & Sample \\
\hline \multirow[t]{5}{*}{ Solids, Percent } & Building 69 & $10 / 17 / 2007$ & 78 & $\%$ & Sample \\
\hline & & $10 / 17 / 2007$ & 77 & $\%$ & Split \\
\hline & Building 80 & $10 / 17 / 2007$ & 75 & $\%$ & Sample \\
\hline & Building 85 & $10 / 17 / 2007$ & 79 & $\%$ & Sample \\
\hline & ENV-B13C & 10/17/2007 & 80 & $\%$ & Sample \\
\hline
\end{tabular}




\begin{tabular}{|c|c|c|c|c|c|}
\hline \multicolumn{2}{|c|}{ Metals } & \multirow[b]{2}{*}{$\begin{array}{c}\text { Collection } \\
\text { Date }\end{array}$} & \multirow[b]{2}{*}{ Result } & \multirow[b]{2}{*}{ Units } & \multirow[b]{2}{*}{ QA Type } \\
\hline Analyte & Location & & & & \\
\hline \multirow[t]{5}{*}{ Aluminum } & Building 69 & $10 / 17 / 2007$ & 30000 & $\mathrm{mg} / \mathrm{kg}$ & Sample \\
\hline & & $10 / 17 / 2007$ & 28000 & $\mathrm{mg} / \mathrm{kg}$ & Split \\
\hline & Building 80 & 10/17/2007 & 16000 & $\mathrm{mg} / \mathrm{kg}$ & Sample \\
\hline & Building 85 & $10 / 17 / 2007$ & 30000 & $\mathrm{mg} / \mathrm{kg}$ & Sample \\
\hline & ENV-B13C & $10 / 17 / 2007$ & 13000 & $\mathrm{mg} / \mathrm{kg}$ & Sample \\
\hline \multirow[t]{5}{*}{ Arsenic } & Building 69 & 10/17/2007 & $<5$ & $\mathrm{mg} / \mathrm{kg}$ & Sample \\
\hline & & 10/17/2007 & $<5$ & $\mathrm{mg} / \mathrm{kg}$ & Split \\
\hline & Building 80 & $10 / 17 / 2007$ & 5.9 & $\mathrm{mg} / \mathrm{kg}$ & Sample \\
\hline & Building 85 & $10 / 17 / 2007$ & $<5$ & $\mathrm{mg} / \mathrm{kg}$ & Sample \\
\hline & ENV-B13C & 10/17/2007 & 8.3 & $\mathrm{mg} / \mathrm{kg}$ & Sample \\
\hline \multirow[t]{5}{*}{ Barium } & Building 69 & 10/17/2007 & 90 & $\mathrm{mg} / \mathrm{kg}$ & Sample \\
\hline & & $10 / 17 / 2007$ & 88 & $\mathrm{mg} / \mathrm{kg}$ & Split \\
\hline & Building 80 & 10/17/2007 & 160 & $\mathrm{mg} / \mathrm{kg}$ & Sample \\
\hline & Building 85 & $10 / 17 / 2007$ & 92 & $\mathrm{mg} / \mathrm{kg}$ & Sample \\
\hline & ENV-B13C & $10 / 17 / 2007$ & 110 & $\mathrm{mg} / \mathrm{kg}$ & Sample \\
\hline \multirow[t]{5}{*}{ Boron } & Building 69 & 10/17/2007 & $<10$ & $\mathrm{mg} / \mathrm{kg}$ & Sample \\
\hline & & $10 / 17 / 2007$ & $<10$ & $\mathrm{mg} / \mathrm{kg}$ & Split \\
\hline & Building 80 & $10 / 17 / 2007$ & $<10$ & $\mathrm{mg} / \mathrm{kg}$ & Sample \\
\hline & Building 85 & 10/17/2007 & $<10$ & $\mathrm{mg} / \mathrm{kg}$ & Sample \\
\hline & ENV-B13C & $10 / 17 / 2007$ & $<10$ & $\mathrm{mg} / \mathrm{kg}$ & Sample \\
\hline \multirow[t]{5}{*}{ Chromium } & Building 69 & $10 / 17 / 2007$ & 73 & $\mathrm{mg} / \mathrm{kg}$ & Sample \\
\hline & & $10 / 17 / 2007$ & 75 & $\mathrm{mg} / \mathrm{kg}$ & Split \\
\hline & Building 80 & $10 / 17 / 2007$ & 30 & $\mathrm{mg} / \mathrm{kg}$ & Sample \\
\hline & Building 85 & $10 / 17 / 2007$ & 77 & $\mathrm{mg} / \mathrm{kg}$ & Sample \\
\hline & ENV-B13C & $10 / 17 / 2007$ & 28 & $\mathrm{mg} / \mathrm{kg}$ & Sample \\
\hline \multirow[t]{5}{*}{ Cobalt } & Building 69 & $10 / 17 / 2007$ & 19 & $\mathrm{mg} / \mathrm{kg}$ & Sample \\
\hline & & $10 / 17 / 2007$ & 18 & $\mathrm{mg} / \mathrm{kg}$ & Split \\
\hline & Building 80 & $10 / 17 / 2007$ & 11 & $\mathrm{mg} / \mathrm{kg}$ & Sample \\
\hline & Building 85 & $10 / 17 / 2007$ & 19 & $\mathrm{mg} / \mathrm{kg}$ & Sample \\
\hline & ENV-B13C & 10/17/2007 & $<10$ & $\mathrm{mg} / \mathrm{kg}$ & Sample \\
\hline \multirow[t]{4}{*}{ Copper } & Building 69 & $10 / 17 / 2007$ & 19 & $\mathrm{mg} / \mathrm{kg}$ & Sample \\
\hline & & $10 / 17 / 2007$ & 20 & $\mathrm{mg} / \mathrm{kg}$ & Split \\
\hline & Building 80 & $10 / 17 / 2007$ & 34 & $\mathrm{mg} / \mathrm{kg}$ & Sample \\
\hline & Building 85 & $10 / 17 / 2007$ & 34 & $\mathrm{mg} / \mathrm{kg}$ & Sample \\
\hline
\end{tabular}




\begin{tabular}{|c|c|c|c|c|c|}
\hline \multicolumn{2}{|c|}{ Metals } & \multirow[b]{2}{*}{$\begin{array}{l}\text { Collection } \\
\text { Date }\end{array}$} & \multirow[b]{2}{*}{ Result } & \multirow[b]{2}{*}{ Units } & \multirow[b]{2}{*}{ QA Type } \\
\hline Analyte & Location & & & & \\
\hline Copper & ENV-B13C & $10 / 17 / 2007$ & 21 & $\mathrm{mg} / \mathrm{kg}$ & Sample \\
\hline \multirow[t]{5}{*}{ Iron } & Building 69 & $10 / 17 / 2007$ & 36000 & $\mathrm{mg} / \mathrm{kg}$ & Sample \\
\hline & & $10 / 17 / 2007$ & 33000 & $\mathrm{mg} / \mathrm{kg}$ & Split \\
\hline & Building 80 & $10 / 17 / 2007$ & 20000 & $\mathrm{mg} / \mathrm{kg}$ & Sample \\
\hline & Building 85 & $10 / 17 / 2007$ & 38000 & $\mathrm{mg} / \mathrm{kg}$ & Sample \\
\hline & ENV-B13C & $10 / 17 / 2007$ & 20000 & $\mathrm{mg} / \mathrm{kg}$ & Sample \\
\hline \multirow[t]{5}{*}{ Lead } & Building 69 & $10 / 17 / 2007$ & $<10$ & $\mathrm{mg} / \mathrm{kg}$ & Sample \\
\hline & & $10 / 17 / 2007$ & 11 & $\mathrm{mg} / \mathrm{kg}$ & Split \\
\hline & Building 80 & $10 / 17 / 2007$ & 97 & $\mathrm{mg} / \mathrm{kg}$ & Sample \\
\hline & Building 85 & $10 / 17 / 2007$ & $<10$ & $\mathrm{mg} / \mathrm{kg}$ & Sample \\
\hline & ENV-B13C & $10 / 17 / 2007$ & 54 & $\mathrm{mg} / \mathrm{kg}$ & Sample \\
\hline \multirow[t]{5}{*}{ Magnesium } & Building 69 & $10 / 17 / 2007$ & 11000 & $\mathrm{mg} / \mathrm{kg}$ & Sample \\
\hline & & $10 / 17 / 2007$ & 11000 & $\mathrm{mg} / \mathrm{kg}$ & Split \\
\hline & Building 80 & $10 / 17 / 2007$ & 5300 & $\mathrm{mg} / \mathrm{kg}$ & Sample \\
\hline & Building 85 & $10 / 17 / 2007$ & 9800 & $\mathrm{mg} / \mathrm{kg}$ & Sample \\
\hline & ENV-B13C & $10 / 17 / 2007$ & 3600 & $\mathrm{mg} / \mathrm{kg}$ & Sample \\
\hline \multirow[t]{5}{*}{ Manganese } & Building 69 & $10 / 17 / 2007$ & 590 & $\mathrm{mg} / \mathrm{kg}$ & Sample \\
\hline & & $10 / 17 / 2007$ & 560 & $\mathrm{mg} / \mathrm{kg}$ & Split \\
\hline & Building 80 & $10 / 17 / 2007$ & 640 & $\mathrm{mg} / \mathrm{kg}$ & Sample \\
\hline & Building 85 & $10 / 17 / 2007$ & 670 & $\mathrm{mg} / \mathrm{kg}$ & Sample \\
\hline & ENV-B13C & $10 / 17 / 2007$ & 360 & $\mathrm{mg} / \mathrm{kg}$ & Sample \\
\hline \multirow[t]{5}{*}{ Mercury } & Building 69 & $10 / 17 / 2007$ & $<0.2$ & $\mathrm{mg} / \mathrm{kg}$ & Sample \\
\hline & & $10 / 17 / 2007$ & $<0.2$ & $\mathrm{mg} / \mathrm{kg}$ & Split \\
\hline & Building 80 & $10 / 17 / 2007$ & $<0.2$ & $\mathrm{mg} / \mathrm{kg}$ & Sample \\
\hline & Building 85 & $10 / 17 / 2007$ & $<0.2$ & $\mathrm{mg} / \mathrm{kg}$ & Sample \\
\hline & ENV-B13C & $10 / 17 / 2007$ & $<0.2$ & $\mathrm{mg} / \mathrm{kg}$ & Sample \\
\hline \multirow[t]{5}{*}{ Nickel } & Building 69 & $10 / 17 / 2007$ & 47 & $\mathrm{mg} / \mathrm{kg}$ & Sample \\
\hline & & $10 / 17 / 2007$ & 46 & $\mathrm{mg} / \mathrm{kg}$ & Split \\
\hline & Building 80 & $10 / 17 / 2007$ & 35 & $\mathrm{mg} / \mathrm{kg}$ & Sample \\
\hline & Building 85 & $10 / 17 / 2007$ & 60 & $\mathrm{mg} / \mathrm{kg}$ & Sample \\
\hline & ENV-B13C & $10 / 17 / 2007$ & 28 & $\mathrm{mg} / \mathrm{kg}$ & Sample \\
\hline \multirow[t]{4}{*}{ Vanadium } & Building 69 & $10 / 17 / 2007$ & 66 & $\mathrm{mg} / \mathrm{kg}$ & Sample \\
\hline & & $10 / 17 / 2007$ & 63 & $\mathrm{mg} / \mathrm{kg}$ & Split \\
\hline & Building 80 & $10 / 17 / 2007$ & 34 & $\mathrm{mg} / \mathrm{kg}$ & Sample \\
\hline & Building 85 & $10 / 17 / 2007$ & 80 & $\mathrm{mg} / \mathrm{kg}$ & Sample \\
\hline
\end{tabular}




\begin{tabular}{|l|l|c|r|c|c|}
\hline \multicolumn{2}{|c|}{ Metals } & \multicolumn{1}{c|}{} & \\
\hline Analyte & \multicolumn{1}{|c|}{ Location } & $\begin{array}{c}\text { Collection } \\
\text { Date }\end{array}$ & Result & Units & QA Type \\
\hline Vanadium & ENV-B13C & $10 / 17 / 2007$ & 35 & $\mathrm{mg} / \mathrm{kg}$ & Sample \\
\hline Zinc & Building 69 & $10 / 17 / 2007$ & 50 & $\mathrm{mg} / \mathrm{kg}$ & Sample \\
\hline & & $10 / 17 / 2007$ & 53 & $\mathrm{mg} / \mathrm{kg}$ & Split \\
\hline & Building 80 & $10 / 17 / 2007$ & 83 & $\mathrm{mg} / \mathrm{kg}$ & Sample \\
\hline & Building 85 & $10 / 17 / 2007$ & 51 & $\mathrm{mg} / \mathrm{kg}$ & Sample \\
\hline & ENV-B13C & $10 / 17 / 2007$ & 78 & $\mathrm{mg} / \mathrm{kg}$ & Sample \\
\hline
\end{tabular}




\begin{tabular}{|c|c|c|c|c|c|c|c|}
\hline \multicolumn{2}{|c|}{ Radiological Activity } & \multirow[b]{2}{*}{$\begin{array}{c}\text { Collection } \\
\text { Date }\end{array}$} & \multicolumn{2}{|c|}{ S.I. } & \multicolumn{2}{|c|}{ Conventional } & \multirow[b]{2}{*}{$\begin{array}{l}\text { QA } \\
\text { Type }\end{array}$} \\
\hline Analyte & Location & & Result & Units & Result & Units & \\
\hline \multirow[t]{3}{*}{ Actinium 228} & Chicken Creek & $10 / 16 / 2007$ & 0.021 & $\mathrm{~Bq} / \mathrm{g}$ & 0.56 & $\mathrm{pCi} / \mathrm{g}$ & Sample \\
\hline & & $10 / 16 / 2007$ & 0.021 & $\mathrm{~Bq} / \mathrm{g}$ & 0.56 & $\mathrm{pCi} / \mathrm{g}$ & Split \\
\hline & $\begin{array}{l}\text { N. Fork Strawberry } \\
\text { Creek }\end{array}$ & 10/16/2007 & 0.023 & $\mathrm{~Bq} / \mathrm{g}$ & 0.62 & $\mathrm{pCi} / \mathrm{g}$ & Sample \\
\hline \multirow[t]{3}{*}{ Cesium 137} & Chicken Creek & $10 / 16 / 2007$ & 0.0011 & $\mathrm{~Bq} / \mathrm{g}$ & 0.029 & $\mathrm{pCi} / \mathrm{g}$ & Sample \\
\hline & & $10 / 16 / 2007$ & 0.00067 & $\mathrm{~Bq} / \mathrm{g}$ & 0.018 & $\mathrm{pCi} / \mathrm{g}$ & Split \\
\hline & $\begin{array}{l}\text { N. Fork Strawberry } \\
\text { Creek }\end{array}$ & 10/16/2007 & $<0.00052$ & $\mathrm{~Bq} / \mathrm{g}$ & $<0.014$ & $\mathrm{pCi} / \mathrm{g}$ & Sample \\
\hline \multirow[t]{3}{*}{ Gross alpha } & Chicken Creek & $10 / 16 / 2007$ & 0.18 & $\mathrm{~Bq} / \mathrm{g}$ & 4.9 & $\mathrm{pCi} / \mathrm{g}$ & Sample \\
\hline & & $10 / 16 / 2007$ & 0.086 & $\mathrm{~Bq} / \mathrm{g}$ & 2.3 & $\mathrm{pCi} / \mathrm{g}$ & Split \\
\hline & $\begin{array}{l}\text { N. Fork Strawberry } \\
\text { Creek }\end{array}$ & 10/16/2007 & 0.44 & $\mathrm{~Bq} / \mathrm{g}$ & 12 & $\mathrm{pCi} / \mathrm{g}$ & Sample \\
\hline \multirow[t]{3}{*}{ Gross beta } & Chicken Creek & $10 / 16 / 2007$ & 0.5 & $\mathrm{~Bq} / \mathrm{g}$ & 14 & $\mathrm{pCi} / \mathrm{g}$ & Sample \\
\hline & & $10 / 16 / 2007$ & 0.1 & $\mathrm{~Bq} / \mathrm{g}$ & 2.7 & $\mathrm{pCi} / \mathrm{g}$ & Split \\
\hline & $\begin{array}{l}\text { N. Fork Strawberry } \\
\text { Creek }\end{array}$ & 10/16/2007 & 0.64 & $\mathrm{~Bq} / \mathrm{g}$ & 17 & $\mathrm{pCi} / \mathrm{g}$ & Sample \\
\hline \multirow[t]{3}{*}{ Lead 214} & Chicken Creek & $10 / 16 / 2007$ & 0.019 & $\mathrm{~Bq} / \mathrm{g}$ & 0.52 & $\mathrm{pCi} / \mathrm{g}$ & Sample \\
\hline & & $10 / 16 / 2007$ & 0.022 & $\mathrm{~Bq} / \mathrm{g}$ & 0.59 & $\mathrm{pCi} / \mathrm{g}$ & Split \\
\hline & $\begin{array}{l}\text { N. Fork Strawberry } \\
\text { Creek }\end{array}$ & 10/16/2007 & 0.018 & $\mathrm{~Bq} / \mathrm{g}$ & 0.49 & $\mathrm{pCi} / \mathrm{g}$ & Sample \\
\hline \multirow[t]{3}{*}{$\begin{array}{l}\text { Potassium } \\
40\end{array}$} & Chicken Creek & 10/16/2007 & 0.44 & $\mathrm{~Bq} / \mathrm{g}$ & 12 & $\mathrm{pCi} / \mathrm{g}$ & Sample \\
\hline & & $10 / 16 / 2007$ & 0.41 & $\mathrm{~Bq} / \mathrm{g}$ & 11 & $\mathrm{pCi} / \mathrm{g}$ & Split \\
\hline & $\begin{array}{l}\text { N. Fork Strawberry } \\
\text { Creek }\end{array}$ & 10/16/2007 & 0.52 & $\mathrm{~Bq} / \mathrm{g}$ & 14 & $\mathrm{pCi} / \mathrm{g}$ & Sample \\
\hline \multirow[t]{3}{*}{ Radium 226} & Chicken Creek & $10 / 16 / 2007$ & 0.016 & $\mathrm{~Bq} / \mathrm{g}$ & 0.44 & $\mathrm{pCi} / \mathrm{g}$ & Sample \\
\hline & & $10 / 16 / 2007$ & 0.018 & $\mathrm{~Bq} / \mathrm{g}$ & 0.48 & $\mathrm{pCi} / \mathrm{g}$ & Split \\
\hline & $\begin{array}{l}\text { N. Fork Strawberry } \\
\text { Creek }\end{array}$ & 10/16/2007 & 0.017 & $\mathrm{~Bq} / \mathrm{g}$ & 0.46 & $\mathrm{pCi} / \mathrm{g}$ & Sample \\
\hline \multirow[t]{3}{*}{ Radium 228} & Chicken Creek & $10 / 16 / 2007$ & 0.021 & $\mathrm{~Bq} / \mathrm{g}$ & 0.56 & $\mathrm{pCi} / \mathrm{g}$ & Sample \\
\hline & & $10 / 16 / 2007$ & 0.021 & $\mathrm{~Bq} / \mathrm{g}$ & 0.56 & $\mathrm{pCi} / \mathrm{g}$ & Split \\
\hline & $\begin{array}{l}\text { N. Fork Strawberry } \\
\text { Creek }\end{array}$ & 10/16/2007 & 0.023 & $\mathrm{~Bq} / \mathrm{g}$ & 0.62 & $\mathrm{pCi} / \mathrm{g}$ & Sample \\
\hline \multirow[t]{3}{*}{ Tritium } & Chicken Creek & $10 / 16 / 2007$ & $<0.0065$ & $\mathrm{~Bq} / \mathrm{g}$ & $<0.17$ & $\mathrm{pCi} / \mathrm{g}$ & Sample \\
\hline & & $10 / 16 / 2007$ & 0.007 & $\mathrm{~Bq} / \mathrm{g}$ & 0.19 & $\mathrm{pCi} / \mathrm{g}$ & Split \\
\hline & $\begin{array}{l}\text { N. Fork Strawberry } \\
\text { Creek }\end{array}$ & 10/16/2007 & $<0.0067$ & $\mathrm{~Bq} / \mathrm{g}$ & $<0.18$ & $\mathrm{pCi} / \mathrm{g}$ & Sample \\
\hline \multirow[t]{2}{*}{ Uranium 238} & Chicken Creek & $10 / 16 / 2007$ & 0.029 & $\mathrm{~Bq} / \mathrm{g}$ & 0.79 & $\mathrm{pCi} / \mathrm{g}$ & Sample \\
\hline & & $10 / 16 / 2007$ & 0.026 & $\mathrm{~Bq} / \mathrm{g}$ & 0.71 & $\mathrm{pCi} / \mathrm{g}$ & Split \\
\hline
\end{tabular}




\begin{tabular}{|l|c|c|c|c|c|c|c|}
\hline \multicolumn{2}{|c|}{ Radiological Activity } & \multicolumn{2}{c|}{ S.I. } & \multicolumn{2}{c|}{ Conventional } & \\
\hline Analyte & Location & $\begin{array}{c}\text { Collection } \\
\text { Date }\end{array}$ & Result & Units & Result & Units & $\begin{array}{c}\text { QA } \\
\text { Type }\end{array}$ \\
\hline Uranium 238 & $\begin{array}{l}\text { N. Fork Strawberry } \\
\text { Creek }\end{array}$ & $10 / 16 / 2007$ & $<0.019$ & $\mathrm{~Bq} / \mathrm{g}$ & $<0.5$ & $\mathrm{pCi} / \mathrm{g}$ & Sample \\
\hline
\end{tabular}




\begin{tabular}{|l|l|c|c|c|c|}
\hline \multicolumn{1}{|c|}{ General Indicator Parameters } & \multicolumn{1}{c|}{} & \\
\hline Analyte & \multicolumn{1}{|c|}{ Location } & $\begin{array}{c}\text { Collection } \\
\text { Date }\end{array}$ & Result & Units & QA Type \\
\hline Moisture by weight & Chicken Creek & $10 / 16 / 2007$ & 36 & $\%$ & Sample \\
\hline & & $10 / 16 / 2007$ & 20 & $\%$ & Split \\
\hline & $\begin{array}{l}\text { N. Fork Strawberry } \\
\text { Creek }\end{array}$ & $10 / 16 / 2007$ & 21 & $\%$ & Sample \\
\hline pH & Chicken Creek & $10 / 16 / 2007$ & 7.9 & S.U. & Sample \\
\hline & & $10 / 16 / 2007$ & 7.8 & S.U. & Split \\
\hline Solids, Percent & $\begin{array}{l}\text { N. Fork Strawberry } \\
\text { Creek }\end{array}$ & $10 / 16 / 2007$ & 7.9 & S.U. & Sample \\
\hline & Chicken Creek & $10 / 16 / 2007$ & 64 & $\%$ & Sample \\
\hline & & $10 / 16 / 2007$ & 80 & $\%$ & Split \\
\hline & $\begin{array}{l}\text { N. Fork Strawberry } \\
\text { Creek }\end{array}$ & $10 / 16 / 2007$ & 79 & $\%$ & Sample \\
\hline
\end{tabular}




\begin{tabular}{|c|c|c|c|c|c|}
\hline \multicolumn{2}{|r|}{ Metals } & \multirow[b]{2}{*}{$\begin{array}{c}\text { Collection } \\
\text { Date }\end{array}$} & \multirow[b]{2}{*}{ Result } & \multirow[b]{2}{*}{ Units } & \multirow[b]{2}{*}{ QA Type } \\
\hline Analyte & Location & & & & \\
\hline \multirow[t]{3}{*}{ Aluminum } & Chicken Creek & $10 / 16 / 2007$ & 12000 & $\mathrm{mg} / \mathrm{kg}$ & Sample \\
\hline & & $10 / 16 / 2007$ & 9000 & $\mathrm{mg} / \mathrm{kg}$ & Split \\
\hline & N. Fork Strawberry Creek & $10 / 16 / 2007$ & 7100 & $\mathrm{mg} / \mathrm{kg}$ & Sample \\
\hline \multirow[t]{3}{*}{ Arsenic } & Chicken Creek & $10 / 16 / 2007$ & $<5$ & $\mathrm{mg} / \mathrm{kg}$ & Sample \\
\hline & & $10 / 16 / 2007$ & $<5$ & $\mathrm{mg} / \mathrm{kg}$ & Split \\
\hline & N. Fork Strawberry Creek & $10 / 16 / 2007$ & $<5$ & $\mathrm{mg} / \mathrm{kg}$ & Sample \\
\hline \multirow[t]{3}{*}{ Barium } & Chicken Creek & $10 / 16 / 2007$ & 95 & $\mathrm{mg} / \mathrm{kg}$ & Sample \\
\hline & & $10 / 16 / 2007$ & 76 & $\mathrm{mg} / \mathrm{kg}$ & Split \\
\hline & N. Fork Strawberry Creek & $10 / 16 / 2007$ & 63 & $\mathrm{mg} / \mathrm{kg}$ & Sample \\
\hline \multirow[t]{3}{*}{ Boron } & Chicken Creek & $10 / 16 / 2007$ & $<10$ & $\mathrm{mg} / \mathrm{kg}$ & Sample \\
\hline & & $10 / 16 / 2007$ & $<10$ & $\mathrm{mg} / \mathrm{kg}$ & Split \\
\hline & N. Fork Strawberry Creek & $10 / 16 / 2007$ & $<10$ & $\mathrm{mg} / \mathrm{kg}$ & Sample \\
\hline \multirow[t]{3}{*}{ Chromium } & Chicken Creek & $10 / 16 / 2007$ & 45 & $\mathrm{mg} / \mathrm{kg}$ & Sample \\
\hline & & $10 / 16 / 2007$ & 34 & $\mathrm{mg} / \mathrm{kg}$ & Split \\
\hline & N. Fork Strawberry Creek & $10 / 16 / 2007$ & 17 & $\mathrm{mg} / \mathrm{kg}$ & Sample \\
\hline \multirow[t]{3}{*}{ Cobalt } & Chicken Creek & $10 / 16 / 2007$ & 11 & $\mathrm{mg} / \mathrm{kg}$ & Sample \\
\hline & & $10 / 16 / 2007$ & 12 & $\mathrm{mg} / \mathrm{kg}$ & Split \\
\hline & N. Fork Strawberry Creek & $10 / 16 / 2007$ & $<10$ & $\mathrm{mg} / \mathrm{kg}$ & Sample \\
\hline \multirow[t]{3}{*}{ Copper } & Chicken Creek & $10 / 16 / 2007$ & 30 & $\mathrm{mg} / \mathrm{kg}$ & Sample \\
\hline & & $10 / 16 / 2007$ & 16 & $\mathrm{mg} / \mathrm{kg}$ & Split \\
\hline & N. Fork Strawberry Creek & $10 / 16 / 2007$ & 13 & $\mathrm{mg} / \mathrm{kg}$ & Sample \\
\hline \multirow[t]{3}{*}{ Iron } & Chicken Creek & $10 / 16 / 2007$ & 19000 & $\mathrm{mg} / \mathrm{kg}$ & Sample \\
\hline & & $10 / 16 / 2007$ & 13000 & $\mathrm{mg} / \mathrm{kg}$ & Split \\
\hline & N. Fork Strawberry Creek & $10 / 16 / 2007$ & 14000 & $\mathrm{mg} / \mathrm{kg}$ & Sample \\
\hline \multirow[t]{3}{*}{ Lead } & Chicken Creek & $10 / 16 / 2007$ & 24 & $\mathrm{mg} / \mathrm{kg}$ & Sample \\
\hline & & $10 / 16 / 2007$ & 20 & $\mathrm{mg} / \mathrm{kg}$ & Split \\
\hline & N. Fork Strawberry Creek & $10 / 16 / 2007$ & $<10$ & $\mathrm{mg} / \mathrm{kg}$ & Sample \\
\hline \multirow[t]{3}{*}{ Magnesium } & Chicken Creek & $10 / 16 / 2007$ & 7600 & $\mathrm{mg} / \mathrm{kg}$ & Sample \\
\hline & & $10 / 16 / 2007$ & 5400 & $\mathrm{mg} / \mathrm{kg}$ & Split \\
\hline & N. Fork Strawberry Creek & $10 / 16 / 2007$ & 3500 & $\mathrm{mg} / \mathrm{kg}$ & Sample \\
\hline \multirow[t]{3}{*}{ Manganese } & Chicken Creek & $10 / 16 / 2007$ & 450 & $\mathrm{mg} / \mathrm{kg}$ & Sample \\
\hline & & $10 / 16 / 2007$ & 480 & $\mathrm{mg} / \mathrm{kg}$ & Split \\
\hline & N. Fork Strawberry Creek & $10 / 16 / 2007$ & 410 & $\mathrm{mg} / \mathrm{kg}$ & Sample \\
\hline Mercury & Chicken Creek & $10 / 16 / 2007$ & $<0.2$ & $\mathrm{mg} / \mathrm{kg}$ & Sample \\
\hline
\end{tabular}




\begin{tabular}{|c|c|c|c|c|c|}
\hline \multicolumn{2}{|r|}{ Metals } & \multirow[b]{2}{*}{$\begin{array}{c}\text { Collection } \\
\text { Date }\end{array}$} & \multirow[b]{2}{*}{ Result } & \multirow[b]{2}{*}{ Units } & \multirow[b]{2}{*}{ QA Type } \\
\hline Analyte & Location & & & & \\
\hline \multirow[t]{2}{*}{ Mercury } & Chicken Creek & $10 / 16 / 2007$ & $<0.2$ & $\mathrm{mg} / \mathrm{kg}$ & Split \\
\hline & N. Fork Strawberry Creek & $10 / 16 / 2007$ & $<0.2$ & $\mathrm{mg} / \mathrm{kg}$ & Sample \\
\hline \multirow[t]{3}{*}{ Nickel } & Chicken Creek & $10 / 16 / 2007$ & 70 & $\mathrm{mg} / \mathrm{kg}$ & Sample \\
\hline & & $10 / 16 / 2007$ & 43 & $\mathrm{mg} / \mathrm{kg}$ & Split \\
\hline & N. Fork Strawberry Creek & $10 / 16 / 2007$ & 14 & $\mathrm{mg} / \mathrm{kg}$ & Sample \\
\hline \multirow[t]{3}{*}{ Vanadium } & Chicken Creek & $10 / 16 / 2007$ & 32 & $\mathrm{mg} / \mathrm{kg}$ & Sample \\
\hline & & $10 / 16 / 2007$ & 28 & $\mathrm{mg} / \mathrm{kg}$ & Split \\
\hline & N. Fork Strawberry Creek & $10 / 16 / 2007$ & 25 & $\mathrm{mg} / \mathrm{kg}$ & Sample \\
\hline \multirow[t]{3}{*}{ Zinc } & Chicken Creek & $10 / 16 / 2007$ & 120 & $\mathrm{mg} / \mathrm{kg}$ & Sample \\
\hline & & $10 / 16 / 2007$ & 120 & $\mathrm{mg} / \mathrm{kg}$ & Split \\
\hline & N. Fork Strawberry Creek & $10 / 16 / 2007$ & 86 & $\mathrm{mg} / \mathrm{kg}$ & Sample \\
\hline
\end{tabular}




\begin{tabular}{|l|l|c|c|c|c|}
\hline \multicolumn{2}{|c|}{ Petroleum Hydrocarbons } & & & \\
\hline Analyte & \multicolumn{1}{|c|}{ Location } & $\begin{array}{c}\text { Collection } \\
\text { Date }\end{array}$ & Result & Units & QA Type \\
\hline $\begin{array}{l}\text { Diesel Range Organics } \\
\text { (C12-C24) }\end{array}$ & Chicken Creek & $10 / 16 / 2007$ & $<10$ & $\mathrm{mg} / \mathrm{kg}$ & Sample \\
\hline & & $10 / 16 / 2007$ & $<10$ & $\mathrm{mg} / \mathrm{kg}$ & Split \\
\hline & N. Fork Strawberry Creek & $10 / 16 / 2007$ & $<10$ & $\mathrm{mg} / \mathrm{kg}$ & Sample \\
\hline Oil and Grease & Chicken Creek & $10 / 16 / 2007$ & 460 & $\mathrm{mg} / \mathrm{kg}$ & Sample \\
\hline & & $10 / 16 / 2007$ & 290 & $\mathrm{mg} / \mathrm{kg}$ & Split \\
\hline & N. Fork Strawberry Creek & $10 / 16 / 2007$ & 380 & $\mathrm{mg} / \mathrm{kg}$ & Sample \\
\hline
\end{tabular}

